\title{
Abstract \\ Measurement of the Negative Pion and Positive Kaon Total Hadronic Cross Sections on Argon at the LArIAT Experiment
}

\author{
Elena Gramellini
}

2018

The Liquid Argon Time Projection Chamber (LArTPC) represents one of the most advanced experimental technologies for physics at the Intensity Frontier due to its full 3D-imaging, excellent particle identification and precise calorimetric energy reconstruction. By deploying a LArTPC in a dedicated calibration test beam line at Fermilab, LArIAT (Liquid Argon In A Testbeam) aims to experimentally calibrate this technology in a controlled environment and to provide physics results key to the neutrino oscillation physics and proton decay searches of the Short Baseline Neutrino (SBND, MicroBooNE, ICARUS) and Long Baseline Neutrino programs (DUNE).

LArIAT's physics program entails a vast set of topics with a particular focus on the study of nuclear effects such as pion and kaon characteristic interaction modes.

This thesis presents two measurements performed for the first time: the measurement of the negative pion total hadronic cross section on argon in the 100-1050 MeV kinetic energy range and the measurement of the positive kaon total hadronic cross section on argon in the 100-650 MeV kinetic energy range. The analyses devised for these measurements use both the core elements of LArIAT: beamline and LArTPC. The first step in each analysis is the development of an event selection based on beamline and TPC information geared towards the identification of the hadron of interest. We then proceed to match the beamline candidate to a suitable TPC track. Finally, we apply the "thin slice method" technique and measure the cross section, correcting for background contamination and detector effects. The thin slice technique is a new 
method to measure hadron-argon cross sections possible only due to the combination of the tracking and calorimetry capability of the LArTPC technology. Albeit never on argon, the hadronic cross section of pions has been extensively measured before on lighter and heavier different elements in thin target experiments, leading to solid predictions for measurements on argon. Through the use of a different technique, our measurement of the negative pion total hadronic cross section on argon is in general agreement with the predictions by the Geant 4 Bertini Cascade model which are based on data from thin target experiments. On the contrary, cross section measurements for kaons are extremely scarce, thus more difficult to model. Our measurement of the positive kaon total hadronic cross section on argon is mostly in tension with the Geant4 prediction over the explored range of energies and provides new experimental data to properly compare existing interaction models.

This thesis also reports two ancillary detector physics measurements necessary for the cross section analyses: the measurements of the LArIAT electric field and calorimetry constants. We developed a technique to measure the LArIAT electric field using cathode-anode piercing tracks with cosmic data. We applied a new technique for the measurement of the calorimetry calibration constants based on the particles' momentum measurement.

The negative pion and the positive kaon total hadronic cross measurements are the first physics results of the LArIAT experiment and will be the basis for the future LArIAT measurements of pion and kaon cross sections in the exclusive channels. The outcome of these measurements will ultimately enable to quantify and reduce the systematic associated with the hadronic interaction models in neutrino-argon interactions. 


\title{
Measurement of the Negative Pion and Positive Kaon Total Hadronic Cross Sections on Argon at the LArIAT Experiment
}

\author{
A Dissertation \\ Presented to the Faculty of the Graduate School \\ of \\ Yale University \\ in Candidacy for the Degree of \\ Doctor of Philosophy
}

by

Elena Gramellini

Dissertation Director: Bonnie T. Fleming

June 2018 
Copyright (C) 2018 by Elena Gramellini

All rights reserved. 
A mia mamma e mio babbo, grazie per le radici e grazie per le ali.

To my mom and dad, thank you for the roots and thank you for the wings. 


\section{Contents}

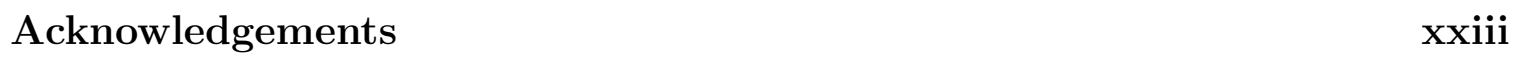

Introduction xxvii

$\begin{array}{lll}1 & \text { The theoretical framework } & 1\end{array}$

1.1 The Standard Model . . . . . . . . . . . . . . . . . . . . . . . . . . . 1

1.2 Neutrinos: tiny cracks in the Standard Model . . . . . . . . . . . . . 4

1.2.1 Neutrinos in the Standard Model . . . . . . . . . . . . . . . . 4

1.2 .2 Neutrino Oscillations . . . . . . . . . . . . 6

1.2 .3 Make up of Neutrino Interactions . . . . . . . . . . . . . 7

1.3 Beyond the Standard Model . . . . . . . . . . . . . . . . . . . . 11

1.3 .1 Open Questions in Neutrino Physics . . . . . . . . . . . . 11

1.3 .2 Towards a more fundamental theory: GUTs . . . . . . . . . . 15

1.4 Motivations for Hadronic Cross Sections in Argon . . . . . . . . . . . 18

1.4 .1 Pion-Argon Total Hadronic Cross Section. . . . . . . . . . . . 19

1.4 .2 Kaon-Argon Total Hadronic Cross Section . . . . . . . . . . . 30

$\begin{array}{|lll|}2 & \text { Liquid Argon Detectors at the Intensity Frontier } & 37\end{array}$

2.1 The Liquid Argon Time Projection Chamber Technology . . . . . . . 37

2.1 .1 TPCs, Neutrinos \& Argon . . . . . . . . . . . . . . . . . . . 38

2.1 .2 LArTPC: Principles of Operation . . . . . . . . . . . . . . . 40 
2.1 .3 Liquid Argon: Ionization Charge . . . . . . . . . . . . . . 43

2.1 .4 Liquid Argon: Scintillation Light $\ldots \ldots \ldots \ldots$

$2.1 .5 \quad$ Signal Processing and Event Reconstruction . . . . . . . . . 52

2.2 The Intensity Frontier Program $\ldots \ldots \ldots \ldots \ldots$

2.2.1 Prospects for LArTPCs in Neutrino Physics: SBN and DUNE 57

2.2 .2 Prospects for LArTPCs in GUT Physics: DUNE. . . . . . . . 59

$2.2 .3 \quad$ Enabling the next generation of discoveries: LArIAT $\ldots . .59$

3 LArIAT: Liquid Argon In A Testbeam 63

3.1 The Particles' Path to LArIAT . . . . . . . . . . . . . . 63

3.2 LArIAT Tertiary Beam Instrumentation $\ldots \ldots \ldots \ldots \ldots$

$3.2 .1 \quad$ Bending Magnets . . . . . . . . . . . . . . . . . . 66

3.2 .2 Multi-Wire Proportional Chambers _... . . . . . . . . 68

3.2 .3 Time-of-Flight System . . . . . . . . . . . . . . . . . 70

$3.2 .4 \quad$ Punch-Through and Muon Range Stack Instruments _. . . . 71

$3.2 .5 \quad$ LArIAT Cosmic Ray Paddle Detectors $\ldots \ldots$. . . . . . 73

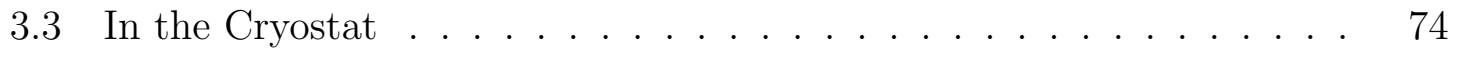

$3.3 .1 \quad$ Cryogenics and Argon Purity $\ldots \ldots \ldots \ldots$

3.3 .2 LArTPC: Charge Collection . . . . . . . . . . . . . 77

$3.3 .3 \quad$ LArTPC: Light Collection . . . . . . . . . . . . . . . . 82

$3.4 \quad$ Trigger and $\mathrm{DAQ} \ldots \ldots \ldots \ldots \ldots$

$3.5 \quad$ Control Systems $\ldots \ldots \ldots \ldots$

\begin{tabular}{|lll}
\hline & Total Hadronic Cross Section Measurement Methodology & 90
\end{tabular}

$4.1 \quad$ Event Selection $\ldots \ldots \ldots \ldots$

4.1 .1 Selection of Beamline Events. . . . . . . . . . . . . . 91

4.1 .2 Particle Identification in the Beamline . . . . . . . . . . 92

4.1 .3 TPC Selection: Halo Mitigation $\ldots \ldots \ldots \ldots$ 
4.1 .4 TPC Selection: Shower Removall. . . . . . . . . . . . . . . . 93

4.2 Beamline and TPC Handshake: the Wire Chamber to TPC Match. . 94

4.3 The Thin Slice Method . . . . . . . . . . . . . . . . . . . . . . 96

$4.3 .1 \quad$ Cross Sections on Thin Target . . . . . . . . . . . . . . 96

4.3 .2 Not-so-Thin Target: Slicing the Liquid Argon Volume. . . . . 97

4.3 .3 Corrections to the Raw Cross Section . . . . . . . . . . . . 100

$4.4 \quad$ Procedure testing with MC truth quantities . . . . . . . . . . 102

$\begin{array}{lll}5 & \text { Data and MC preparation for the Cross Section Measurements } & 104\end{array}$

$5.1 \quad$ Cross Section Analyses Data Sets . . . . . . . . . . . . . . . . 104

5.2 Construction of a Monte Carlo Simulation for LArIAT . . . . . . 106

5.2 .1 G4Beamline . . . . . . . . . . . . . . . . 106

5.2 .2 Data Driven MC . . . . . . . . . . . . . . . . 110

$5.3 \quad$ Estimate of Backgrounds in the Pion Cross Section . . . . . . . . . 112

$5.3 .1 \quad$ Background from Pion Capture and Decay . . . . . . . . . . . 114

5.3 .2 Contributions from the Beamline Background $\ldots \ldots \ldots$. . . 117

5.4 Estimate of Energy Loss before the TPC . . . . . . . . . . . . . . 121

5.5 Tracking Studies $\ldots \ldots \ldots$

5.5 .1 Angular Resolution . . . . . . . . . . . . . . . . . 125

5.5 .2 Track Pitch . . . . . . . . . . . . . . . . . 128

$5.6 \quad$ Calorimetry Studies $\ldots \ldots \ldots \ldots \ldots$

5.6 .1 Kinetic Energy Measurement . . . . . . . . . . . . . . 132

$\begin{array}{lll}6 & \text { Negative Pion Cross Section Measurement } & 135\end{array}$

6.1 Raw Cross Section . . . . . . . . . . . . . . . . . . . . 135

$6.1 .1 \quad$ Statistical Uncertainty $\ldots \ldots \ldots \ldots$

6.1 .2 Treatment of Systematics . . . . . . . . . . . . . . . 139

6.2 Corrections to the Raw Cross Section . . . . . . . . . . . . . . 140 
$6.2 .1 \quad$ Background subtraction . . . . . . . . . . . . . . . . . 141

6.2 .2 Correction for Reconstruction Effects . . . . . . . . . . . . . . 142

6.3 Results . . . . . . . . . . . . . . . . . . . . . . . . . . . . . . . . . . . . . . . . 148

7 Positive Kaon Cross Section Measurement 152

7.1 Raw Cross Section . . . . . . . . . . . . . . . . . . . . . . 152

7.2 Corrections to the Raw Cross Section . . . . . . . . . . . . . . . . . . 154

7.3 Results . . . . . . . . . . . . . . . . . . . . . . . . . . 158

$\begin{array}{lll}8 \text { Conclusions } & 160\end{array}$

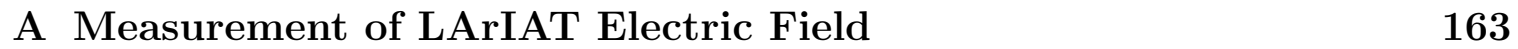

B Additional Tracking Studies for LArIAT Cross Section Analyses 171

B.1 Study of WC to TPC Match . . . . . . . . . . . . . . . . . . . . . . 172

B.2 Tracking Optimization . . . . . . . . . . . . . . . . . . 174

\begin{tabular}{ll}
\hline C Energy Calibration & 177
\end{tabular}

\begin{tabular}{ll}
\hline Bibliography & 182
\end{tabular} 


\section{List of Figures}

1.1 Total neutrino (top) and antineutrino (bottom) CC cross sections per nucleon divided by neutrino energy as a function of energy as reported

\begin{tabular}{|c|}
\hline in \\
\hline
\end{tabular} 4 \mid . Predictions for the total (black), the QE (red), resonant (blue) and DIS (green) are provided by the NUANCE generator. The quasielastic scattering data and predictions have been averaged over neutron and proton targets (isoscalar target). . . . . . . . . . . . 10

1.2 Evolution of the inverse of the three coupling constants in the Standard Model as a function of the momentum transferred, $\mid 109]$.$] . . . . . . . 16$

1.3 Pion-Argon interaction topologies due to elastic scattering, inelastic scattering, pion absorption with emission of protons, charge exchange and production of pions as seen in LArIAT data (induction plane only). 22

1.4 Pion-nucleus total cross sections: $\sigma_{\pi^{+}}$for positive pions (right) and $\sigma_{A V}$ (left) for the average between positive and negative pions $\sigma_{A V}=$ $\frac{\sigma_{\pi^{+}+\sigma_{\pi^{-}}}}{2}$ in the $\Delta$ resonance region. The error bars include estimates of systematic uncertainties. The curves are the results of fits to the data assuming a Breit-Wigner shape. This summary plot is reported

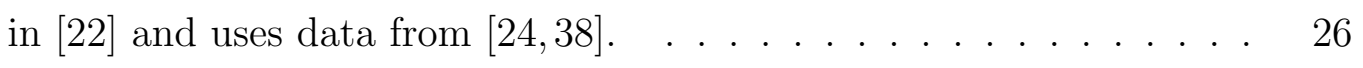

1.5 Negative pion nucleus total, elastic and reaction cross sections on ${ }^{12} \mathrm{C}$

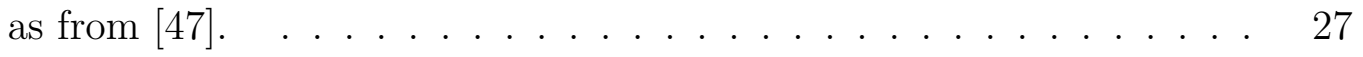


1.6 Total, elastic and reaction cross section for $\pi^{-}$on ${ }^{12} \mathrm{C}$. Comparison between results from Geant4 simulation (Bertini cascade model), GENIE simulation (hA model), and experimental data $[24,[27,39,[19]$.$] . . . . 28$

1.7 Total, elastic and reaction cross section for $\pi^{-}$on ${ }^{40} \mathrm{Ca}$. Comparison between results from Geant4 simulation (Bertini cascade model), GENIE simulation (hA model), and experimental data [27]. . . . . . . 28

1.8 Total, elastic and reaction hadronic cross section for $\pi^{-}$-argon implemented in Geant4 10.01.p3 using FTBF_BERT physics list. . . . . . . 29

1.9 Feynman diagrams for proton decay "golden modes": $p \rightarrow K^{+} \bar{\nu}$ for supersymmetric GUTs on the left and $p \rightarrow e^{+} \pi^{0}$ for gauge-mediated GUTs on the right. . . . . . . . . . . . . . . . . . . . . 31

1.10 Kaon-Argon interaction topologies due to elastic scattering and inelastic scattering as seen in LArIAT data (collection plane only). . . . . . 32

1.11 Ratios between experimental and calculated cross sections as from $[44 \mid$. Top: Total cross sections. Bottom: reaction cross sections. . . . . . . 34

1.12 Total $\mathrm{K}^{+}$and $\mathrm{K}^{-}$cross sections on carbon as from [43]. . . . . . . . . 34

1.13 Total hadronic cross section for carbon implemented in Geant4 10.01.p3 with overlaid with the Bugg and Frideman data. . . . . . . . . . . . . 36

1.14 Total, elastic and reaction hadronic cross section for $K^{+}$-argon implemented in Geant4 10.01.p3. . . . . . . . . . . . . . . . . . . . . 36

$2.1 \quad$ Event display of a $\nu_{\mu} \mathrm{CC}$ interaction candidate in the MicroBooNE detector. . . . . . . . . . . . . . . . . . . . 40

2.2 A cartoonish sketch of a LArTPC. . . . . . . . . . . . . . . . . 41

$2.3 \quad$ Event display for a LArIAT pion absorption candidate on the induction plane, with highlighted delta ray. . . . . . . . . . . . . . . . . . . 44

2.4 Electron lifetime during the LArIAT run period $[11] . . . . . . . \quad 45$ 
2.5 Emission spectra of the fast and slow emission components in Xenon, Kypton and Argon according to $[45]$. The dotted lines correspond to the Gaussian fits. . . . . . . . . . . . . . . . . . . . . . . . . . . . . 49

$2.6 \quad$ A scheme of a typical signal processing workflow in LArSoft. . . . . . 53

$2.7 \quad$ Proton decay lifetime limits from passed and future experiments. . . . 60

2.8 Left. Simulation of the products of a $\nu_{e}$ CC interaction in DUNE, both in particles type and momentum. Right. Momentum spectrum for low mass particles $(e, \mu, \pi)$ in the LArIAT beamline, negative tune, Run II, Picky Tracks see section $|3.2 .2|$. . . . . . . . . . . . . . . . . 61

2.9 Left. Momentum of the kaon outgoing a proton decay $p \rightarrow K^{+} \bar{\nu}$ event as simulated by the Genie 2.8.10 event generator in argon. The red line represents the kaon momentum distribution before undergoing the simulated final state interaction inside the argon nucleus, while the blue line represents the momentum distribution after FSI. Right. Positive Kaon momentum spectrum in the LArIAT beamline, positive tune, Run II, Picky Tracks see section $|3.2 .2|$. . . . . . . . . . . . . . 62

3.1 Layout of Fermilab Accelerator complex. . . . . . . . . . . . . . 65

3.2 Bird's eye view of the LArIAT tertiary beamline. In grey: upstream and downstream collimators; in yellow: bending magnets; in red: multi wire proportional chambers; in blue: time of flight; in green: liquid argon TPC volume; in maroon: muon range statck. . . . . . . . . . . 66

3.3 Magnetic field over current as a function of the current, for one NDB magnet (excitation curve). The data were collected using two hall probes (blue and green). We fit the readings with a cubic function (black) to average of measurements (red) given in the legend $\mid 11]$. . . 67 3.4 One of the four Multi-Wire Proportional Chambers (WC) used in the LArIAT tertiary beamline and related read-out electronics. . . . . . 68 
3.5 Image of the down stream time of flight paddle, PMTs and relative support structure before mounting. . . . . . . . . . . . . . . . . . . . 72

3.6 Photograph of one of the scintillation counters used in the cosmic tow-

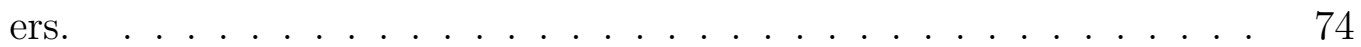

3.7 Left: the LArIAT TPC in the inner volume of the open cryostat. Right: cryostat fully sealed ready to be transported to FTBF. . . . . . . . . 75

3.8 Main modifications to the ArgoNeuT cryostat: 1) outlet for connection to the purification system at the bottom of the cryostat; 2) the "beamwindow" on the outer endcap and "excluder" which reduces the amount of non-instrumented material before the TPC; 3) the side port to host the light collection system. . . . . . . . . . . . . . . . . . . . . . . . 76

3.9 Schematic of the LArIAT high voltage system. . . . . . . . . . . . . . 78

3.10 Schematic of the three drift regions inside the LArIAT TPC: the main drift volume between the cathode and the shield plane (C-S) in green, the region between the shield plane and the induction plane (S-I) in purple, and the region between the induction plane and the collection plane $(\mathrm{I}-\mathrm{C})$ in pink. . . . . . . . . . . . . . . . . . . . . 80

3.11 Overview of LArIAT Front End electronics. . . . . . . . . . . . . . . 81

3.12 LArIAT's photodetector system for observing LAr scintillation light inside the TPC (left), and a simplified schematic of VUV light being wavelength-shifting along the TPB-coated reflecting foils (right). . . . 82

3.13 Interface of the Synoptic slow control system . . . . . . . . . . . . . . 86 3.14 Beam position at the upstream wire chambers monitored with ACNET. 87 
3.15 Run Status page at LArIAT downtime. At the top the yellow bar displays the current position in the Fermilab supercycle. Interesting information to be monitored by the shifter were the run number and number of spills, time elapsed from data taking (here in red), the energy of the secondary beam and the trigger paths. . . . . . . . . . . . 88

3.16 High occupancy event display: induction plane (top) and collection plane (bottom). . . . . . . . . . . . . . . . . . . 89

4.1 Distribution of the beamline mass as calculated according to equation \begin{tabular}{|l|l|l|l}
\hline & for the Run-II events reconstructed in the beamline, negative po-
\end{tabular} larity runs on the left and positive polarity runs on the right. The classification of the events into $\pi^{ \pm} / \mu^{ \pm} / e^{ \pm}, \mathrm{K}^{ \pm}$, or (anti)proton is based on these distributions, whose selection cut are represented by the vertical colored lines. . . . . . . . . . . . . . . . . . . . . . . . . . . . . . . . 93

4.2 Visual rendering of the shower filter. The ROI is a cut cone, with a small radius of $4 \mathrm{~cm}$, a big radius of $10 \mathrm{~cm}$ and an height of $42 \mathrm{~cm}$ (corresponding to 3 radiation lengths for electrons in Argon). . . . . . 94

4.3 Kaon candidate event: on the right, event display showing raw quantities; on the left, event display showing reconstructed tracks. In the reconstructed event display, different colors represent different track objects. A kink is visible in the kaon ionization, signature of a hadronic interaction: the tracking correctly stops at the kink position and two tracks are formed. An additional pile-up track is so present in the event (top track in red). . . . . . . . . . . . . . . . . . 95

4.4 Visual rendering of the wire chamber to TPC match. . . . . . . . . . 96

4.5 Representation of sliced LAr Volume. . . . . . . . . . . . . . . . . . . 98 
4.6 Hadronic cross sections for $\left(\pi^{-}, \mathrm{Ar}\right)$ on the left and $\left(\mathrm{K}^{+}, \mathrm{Ar}\right)$ on the right as implemented in Geant4 10.03.p1 (solid lines) overlaid the true MC cross section as obtained with the sliced TPC method (markers). The total cross section is shown in green, the elastic cross section in blue and the inelastic cross section in red. . . . . . . . . . . . . . . . 103

5.1 Beam composition for the -60A runs (left) and -100A runs (right). The solid blue plot represents the simulated pion content, the yellow plot represents the simulated muon content and the grey plot represents the simulated electron content. The plots are area normalized to the number of data events, shown in red. . . . . . . . . . . . . . . . . . . 108

5.2 Left: Beam composition for the +100A runs after WC4 (no mass selection applied). The solid colors represent the contributions from the G4Beamline simulated particles: blue plot represents the simulated pion content, the yellow plot represents the simulated muon content and the grey plot represents the simulated positron content, the red the proton content and the mustard the kaon content. The plots are area normalized to the number of data events, shown in black. Right: Mass distribution for the Run-II positive runs, where the area under the kaon mass peak is highlighted in purple. The area under the extension of a possible fit for the proton tail is highlighted in red. . . . . . . . . . . 110

5.3 Workflow for Data Driven single particle Monte Carlo production. . . 112

5.4 Scheme of the quantities of interest for the DDMC event generation: $P_{x}, P_{y}, P_{z}, X, Y$ at WC4. . . . . . . . . . . . . . . . . 112

5.5 Comparison between generated quantities and data distributions for the 100A kaon sample: Z component of the momentum at WC4 (top left), X position at Wire Chamber 4 (top right), Y position at Wire Chamber 4 (bottom). . . . . . . . . . . . . . . . . . 113 
5.6 MC momentum distribution at wire chamber 4 for every simulated pion arriving in the TPC (pink), ending its life in capture (green) or in decay (blue) in the TPC, linear vertical axis on the left, logarithmic on the right. . . . . . . . . . . . . . . . . . . 115

5.7 Survival ratio as a function of selection threshold on MC momentum at wire chamber four for for every simulated pion arriving in the TPC (pink), capture (green) or in decay (blue). . . . . . . . . . . . . . . . 116

5.8 Ratio between the capture (green) and decay (blue) events over the total number of events as a as a function of the MC momentum at wire chamber four. . . . . . . . . . . . . . . . . . . . . . 116

5.9 Left: staggered contributions to the interacting kinetic energy distribution for electron (grey), muons (yellow) and pion (blue) in the 60A simulation sample. Right: staggered contributions to the incident kinetic energy distribution for electron (grey), muons (yellow) and pion (blue) in the 60A simulation sample. . . . . . . . . . . . . . . . . . . 119

5.10 Left: staggered contributions to the interacting kinetic energy distribution for electron (grey), muons (yellow) and pion (blue) in the 100A simulation sample. Right: staggered contributions to the incident kinetic energy distribution for electron (grey), muons (yellow) and pion (blue) in the 100A simulation sample. . . . . . . . . . . . . . . . . . 119

5.11 True energy loss between WC4 and the TPC front face according to the MC simulation of negative pions of the $60 \mathrm{~A}$ runs (left) and of the 100A runs (right). The distribution for the whole data sample is shown in blue, the distribution for the pions missing the halo is shown in red, and the distribution for the pions hitting the halo is shown in green. 
5.12 True energy loss between WC4 and the TPC front face according to the MC simulation of positive kaons in the $60 \mathrm{~A}$ and $100 \mathrm{~A}$ combined sample. The distribution for the whole data sample is shown in blue, the distribution for the kaons missing the halo is shown in red, and the distribution for the kaons hitting the halo is shown in green. . . . . . 124

5.13 Schematic rendering of the particle path between WC4 and the TPC front face. The paddle with the hollow central circle represents the Halo paddle. We illustrate two possible trajectories: in black, a trajectory that misses the paddle and goes through the hole in the Halo, in blue a trajectory that hits the Halo paddle and goes through the scintillation material. . . . . . . . . . . . . . . . . . . 124

5.14 Horizontal component of the true momentum vs the horizontal position at WC4 for MC simulated pions of the 60A runs. The plot on the left shows the distribution for pion that miss the halo paddle and the plot on the right shows the distributions for pions that hit the halo. The form of the classifier is overlaid to both plots (red line). . . . . . . . . 125

5.15 A visual representation of the procedure used to evaluate the angular resolution. . . . . . . . . . . . . . . . . . . . . . 129

5.16 A visual representation of the scattering angle definition in case of inelastic scattering. . . . . . . . . . . . . . . . . . . . . . . . . . . . . 129

5.21 Left: True $\left(\pi^{-}, A r\right)$ cross section for interaction angles greater than 0 deg (green), $4.5 \mathrm{deg}$ (red), $5.0 \mathrm{deg}$ (blue) and $9.0 \mathrm{deg}$ (yellow). Right: Ratio between the true cross section for interaction angles greater than 5 deg and the true interaction cross section for all angles. . . . . . . . 131

5.22 Left: Pitch distribution for the pion combined sample 60A and 100A, data displayed in black, MC in red. Right: Pitch distribution for the kaon sample, data displayed in black, MC in red. . . . . . . . . . . . 131 
6.1 Raw number of interacting pion candidates as a function of the reconstructed kinetic energy for the 60A runs (left) and for the 100A runs (right). The statistical uncertainties are shown in black, the systematic uncertainties in red. . . . . . . . . . . . . . . . . . . . . . . . 138

6.2 Raw number of incident pion candidates as a function of the reconstructed kinetic energy for the 60A runs (left) and for the 100A runs (right). The statistical uncertainty is shown in black, the systematic uncertainties in red. . . . . . . . . . . . . . . . . . . . 138

6.3 Raw $\left(\pi^{-}\right.$-Ar) total hadronic cross section for the $60 \mathrm{~A}$ runs (left) and for the $100 \mathrm{~A}$ runs (right). The statistical uncertainty is shown in black, the systematic uncertainties in red. The raw cross section obtained with a MC mixed sample of pions, muon and electrons in the percentage predicted by G4Beamline is shown in azure. . . . . . . . . . . . . . . 139

6.4 Left: MC estimated relative pion content for interacting histogram a function of kinetic energy for the 60A runs (top) and 100A runs (bottom), predicted background content in azure and muon and electron content variation in blue and red. Right: MC estimated relative pion content for incident histogram a function of kinetic energy for the $60 \mathrm{~A}$ runs (top) and 100A (bottom), predicted background content in azure and muon and electron content variation in blue and red . . . . . . . 143

6.5 Left: Reconstruction effects correction on the 60A interacting histogram, statistical uncertainty in blue, systematic uncertainty in red. Right: Reconstruction effects correction on the 60A incident histogram, statistical uncertainty in blue, systematic uncertainty in red. . . . . . 146 
6.6 Left: Reconstruction effects correction on the 100A interacting histogram, statistical uncertainty in blue, systematic uncertainty in red. Right: Reconstruction effects correction on the 100A incident histogram, statistical uncertainty in blue, systematic uncertainty in red. 147

6.7 Distribution of the true scattering angle for a pion elastic scattering off the argon nucleus as simulated by Geant4. . . . . . . . . . . . . . 147

6.8 Top Left: $\left(\pi^{-}\right.$-Ar $)$total hadronic cross section for scattering angles greater than $5^{\circ}$ measured in the $60 \mathrm{~A}$ sample, statistical uncertainty and systematic uncertainty in red. The FTFP_BERT Geant4 prediction for the total hadronic cross section for angle scattering greater than $5^{\circ}$ is displayed in green. Top Right: $\left(\pi^{-}-\mathrm{Ar}\right)$ total hadronic cross section for scattering angles greater than $5^{\circ}$ measured in the $100 \mathrm{~A}$ sample, statistical uncertainty and systematic uncertainty in blue. The FTFP_BERT Geant4 prediction for the total hadronic cross section for angle scattering greater than $5^{\circ}$ is displayed in green. Bottom: $\left(\pi^{-}-\right.$ Ar) total hadronic cross section measurements in the $60 \mathrm{~A}$ and $100 \mathrm{~A}$ samples overlaid with the FTFP_BERT Geant4 prediction (green). . . 150

6.9 Top: $\left(\pi^{-}-\mathrm{Ar}\right)$ total hadronic cross section for scattering angles greater than $5^{\circ}$ measured in the combined sample, statistical uncertainty and systematic uncertainty in black. The Geant4 prediction for the total hadronic cross section for angle scattering greater than $5^{\circ}$ is displayed in green. Bottom: Relative difference between the measured cross section and the Geant4 prediction. . . . . . . . . . . . . . . . . . 151

7.1 Raw number of interacting kaon candidates as a function of the reconstructed kinetic energy. The statistical uncertainties are shown in black, the systematic uncertainties in red. . . . . . . . . . . . . . . . 155 
7.2 Raw number of incident kaon candidates as a function of the reconstructed kinetic energy. The statistical uncertainty is shown in black, the systematic uncertainties in red. . . . . . . . . . . . . . . . . . . . 155

7.3 Raw $\left(K^{+}-\right.$Ar) total hadronic cross section. The statistical uncertainty is shown in black, the systematic uncertainties in red. The raw cross section obtained with a MC sample of kaons is shown in azure. For the MC cross section, we include the contributions from secondaries. 155

7.4 Left: MC estimated relative kaon content for kaons interacting hadronically as function of kinetic energy. Right: MC estimated relative kaon content for incident histogram a function of kinetic energy. . . . . . . 157

7.5 Left: Reconstruction effects correction on the interacting histogram, statistical uncertainty in blue, systematic uncertainty in red. Right: Reconstruction effects correction on the incident histogram, statistical uncertainty in blue, systematic uncertainty in red. . . . . . . . . . . . 157

7.6 Top: $\left(K^{+}-\mathrm{Ar}\right)$ total hadronic cross section for scattering angles greater than $5^{\circ}$ measured in the combined $+60 \mathrm{~A}$ and $+100 \mathrm{~A}$ data samples, statistical uncertainty and systematic uncertainty in black. The Geant4 prediction for the total hadronic cross section for angle scattering greater than $5^{\circ}$ is displayed in green. Bottom: Relative deviation between the measured cross section and the Geant4 prediction. . . . . . 159

8.1 Left: $\left(\pi^{-}-\mathrm{Ar}\right)$ total hadronic cross section measurement for scattering angles greater than $5^{\circ}$ measured in the combined sample overlaid with the Geant4 prediction (green). Right: $\left(K^{+}-\mathrm{Ar}\right)$ total hadronic cross section for scattering angles greater than $5^{\circ}$ measured. The respective Geant4 prediction for the total hadronic cross section for angle scattering greater than $5^{\circ}$ is displayed in green. . . . . . . . . . . . 162 
A.1 Drift velocity dependence on electric field for several temperatures. The slope of the line at any one point represents the electron mobility for that given temperature and electric field. . . . . . . . . . . . . . . 164

A.2 LArIAT HV simple scheme. . . . . . . . . . . . . . . . . . . . . . . 165

A.3 Current reading from the Glassman between May 25th and May 30th, 2016 (typical Run-II conditions). . . . . . . . . . . . . . . . . 165

A.4 Pictorial representation of the YX view of the TPC. The distance within the anode planes and between the shield plane and the cathode is purposely out of proportion to illustrate the time difference between hits on collection and induction. An ACP track is shown as an example.169

A.5 Angle definition in the context of LArIAT coordinate system. . . . . . 169

A.6 Collection plane $\Delta$ t fit for Run II positive polarity ACP data selected tracks . . . . . . . . . . . . . . . . . . 170

A.7 Induction plane $\Delta$ t fit for Run II positive polarity ACP data selected tracks. . . . . . . . . . . . . . . . . . 170

B.1 Efficiency (left) and purity (right) for WC2TPC match as a function of the radius and angle selections for the kaon sample. . . . . . . . . . 174

B.2 Example of a shallow angle hadronic interaction "missed" by the TrajCluster. . . . . . . . . . . . . . . . 176

C.1 Stopping power for pions, muons, kaons, and protons in liquid argon over the momentum range most relvant for LArIAT according to the Beth-Bloch equation. The solid lines represent the prediction for the mean energy $d E / d X$, while the dashed lines are the predictions for the MPV. . . . . . . . . . . . . . . . . . . . . . 180 
C.2 Stopping power versus Momentum for Run-II negative (top) and positive (bottom) polarity data. We achieve the agreement between the Bethe-Bloch predictions and the distribution obtained with of the positive pions (top plot, red dots) by tuning the calorimetry constants. Once the calorimetry constants are locked in, the agreement between the other particle species and the Bethe-Bloch predictions follows naturally. . . . . . . . . . . . . . . . . . . . . . . . . . . . . . . . . 180

C.3 Left: $\mathrm{dE} / \mathrm{dx}$ distribution for $\pi^{-} / \mu^{-} / e^{-}$data (black) and Pion MC (blue). The Landau fit for data is shown in red, the one for MC in teal. Right: $\mathrm{dE} / \mathrm{dx}$ distribution for $K^{+}$data (black) and Kaon MC (blue). The Landau fit for data is shown in red, the one for MC in teal. All the distributions are area normalized. . . . . . . . . . . . . . 181 


\section{List of Tables}

1.1 SM elementary fermionic fields. The subscripts L and R indicate respectively the negative chirality (left-handed) and the positive chirality

(right-handed). . . . . . . . . . . . . . . . . . . . . 3

1.2 Summary of experimental results on neutrino oscillation parameters

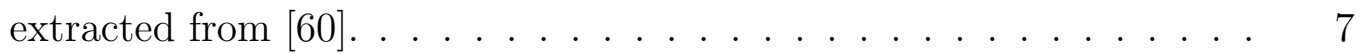

$1.3 \quad$ Main decay modes of the lightest Delta resonance and Nucleon excited state. . . . . . . . . . . . . . . . . 20

1.4 Summary of negative pion hadronic interactions of the reaction channel as a function of the pion multiplicity in the final state for the energy range $[100-1200] \mathrm{MeV}$.

$2.1 \quad$ LAr, LXe summary of properties relevant for neutrino detectors. . . . 39

3.1 Cathode and anode planes default voltages . . . . . . . . . . . . . . 79

$4.1 \quad$ Number of data events for Run-II Negative and Positive polarity . . 91

$4.2 \quad$ Fiducial volume boundaries used to determine cross section interaction point. . . . . . . . . . . . . . . . . . . 100

5.1 Number of data events which fit the $\pi / \mu / e$ or $K$ mass hypothesis as a function of magnet settings. . . . . . . . . . . . . . . . . . . . . . . 106

$5.2 \quad$ Simulated beamline composition per magnet settings . . . . . . . . . 108

5.3 MC selection flow per particle species. . . . . . . . . . . . . . . . 118 
5.4 LArIAT material budget from WC4 to the TPC Front Face. . . . . . 121

$5.5 \quad$ Energy loss for pions and kaons. . . . . . . . . . . . . . . . . . . 123

5.6 Angular resolution for Pion and Kaon tracking in both data and MC. 127

6.1 Beam composition variation for the study of systematics due to beam contamination. . . . . . . . . . . . . . . . 146

A.1 Electric field and drift velocities in LArIAT smaller drift volumes . . 164

A.2 $\Delta t$ for the different data samples used for the Anode-Cathode Piercing tracks study. 


\section{Acknowledgements}

"Dunque io ringrazio tutti quanti.

Specie la mia mamma

che mi ha fatto cosi funky."
"So, I thank everyone. Especially my mom who made me so funky." - Articolo 31, Tanqi Funky, 1996 -

I jumped the pond a little less than 5 years ago because I had a taste of what being a physicist in the US might be like and wanted more. I came to this vast and sometimes inhospitable land thinking I'd learn a lot of physics (which I did) and hoping I'd keep doing research for as long as I could (which I'm fortunate enough to say I'll still do for a while). Little did I know that getting my $\mathrm{PhD}$ would be first and foremost a journey to self discovery, the beginning of a path to asymptotically tend to the person and the scientist I want to be. A journey impossible without the help of many people, which I will now proceed to thank. Let's hope that the acknowledgements won't be longer than the thesis itself.

Mom, you are and will always be the hero of my story; I know this has been challenging for you, thanks for being there for me at every hour of the day and the night, for being always honest and for somehow handling this whole US-Italy thing gracefully. I can't believe you still haven't met Bonnie. Ah, and don't worry, I'll always translate things for you.

Bonnie Fleming, my advisor, I don’t even know where to start in thanking you. Thanks for listening to me before I proved I was worth be listened to; thanks for 
always believing I could make it, even at the bottom of my doubts; thanks for all the laughters, for all the freedom, for all the great advices; thanks for leading by example; thanks for being always supportive of all my crazy side projects; thanks for choosing each and every person in the most wonderful research group ever and thanks for letting me be part of it. Thanks for showing me that even Bonnie Fleming is human, has her downs and still she thrives.

Flavio Cavanna, my co-advisor, working with you has been one of the greatest pleasures of my academic life; the extent of your knowledge never ceases to amaze me and the freshness of your enthusiasm is always contagious. Thanks for all the time spent in your office banging our heads on these cross section analyses and thanks for convincing me to join LArIAT in the first place.

Ornella Palamara, after a particularly heated exchange at a wine and cheese, I once told Andrzej I think of you as my spirit animal: fierce, knowledgeable and direct; he replied I still need to walk many miles to get there and I can't agree more.

A big thank you to my academic sisters, from the youngest to the oldest (again academically speaking): Supraja Balasubramanian, you worked the magic of enriching my past, thank you from the bottom of my heart; Brooke Morrison, the depth of your knowledge, your drive and love for our field has been a constant inspiration; Ariana Hackenburg, thanks for keeping me sane for the last four years, if you run for president - as you should - remember to delete our Skype chats, love and hugs to you dudette; and last but not least Xiao Luo, Fermilab has given me lots of extraordinary gifts, but no one bigger than your friendship.

Jen Raaf and Jonathan Asaadi, these analyses would have not seen the light of day without you. Thanks for your many inputs and for letting your door open to talk about physics and/or my latest paranoia. Thanks for the patience and understanding. And thanks for leading that small experiment with a big heart called LArIAT and all its crazy ponies. Speaking of which, thanks to all my collaborators, especially Irene 
Nutini, who started the path for the pion analysis, Greg Pulliam, who suffered with me at the end of said path, Dan the Man, Jason, Johnny, Will Fo and Mr Bojangles. A special token of gratitude goes to Roberto Acciarri and Andrzej M. Szelc, you are so much more to me than collaborators and previous car owners, you know you'll always have a home in Chicago (or wherever this job will bring me).

Thanks to the MicroBooNE experiment which has been an incredible learning experience. I want to thank Sam Zeller for the countless pieces of advice, Jessica Esquivel, whose passion and resilience I deeply admire, my CRT troopers Martin, David, Rui, Igor and Linda - if I know something about hardware it's because of you - and Kazu and Kirby, who I shamelessly bothered many times for software help, sushi and coffee.

To all the members of the NPG, thanks for all the laughters and all the wise insights. You're the smartest bunch of idiots I've ever met, I love y'all.

Shany Danieli, Louise Laage and Flor Orosz Hunziker, you're the piece of heart I left in New Haven. Shany, I don't know what made you think I was the cool kid in the group, but I'm so glad you stuck with me. I'm not sure I would have survived my first semester at Yale without you. Your intelligence is matched only by your kindness: you have the rare gift to shine and make others shine with you. Louise, my dear, thanks for always finding the time to listen, for your attuned spirit and for the magnolias. Flor, thanks for welcoming me to the new world with love and providing me with the correct definition of "America".

Thanks to all the people which helped me make Chicago and Fermilab my new home, in particular David Caratelli, Mateus Carneiro, Pilar Coloma, Ciaran Hughes, Gordan Krnjaic, Seyda Ipek, Kiel Howe, Adi Ashkenazi and Rob Fine.

To the wonderful people who stayed close even with an ocean in between: la Tasso, Marci, la Bea, la Kittirn, Alvin, la Viola e Brogna, Sighi, la Secchi and the people from the "La Chiappia Nuova" group, thanks for making me feel like I never left. 
Finally, thanks to Anto, my husband, my topo and my adventure companion, cause people might think I'm nice and easy, but you know I'm not... And still, you followed me across half the world and you're here, so that I can never feel alone. 


\section{Introduction}

The discovery of the Higgs boson in 2012 marked the triumph of the Standard Model of Particle Physics; exploring what lays beyond is the real challenge in our field today. Since their formulation in 1930, neutrinos have been a source of surprises (and Nobel Prizes) for particle physicists, tiny cracks in our understanding of Nature. In particular, the discovery of neutrino oscillation represents the first evidence of physics Beyond the Standard Model (BSM). From a theoretical point of view, the field is developing new theories to account for the small but non-zero mass of neutrinos, while trying to remain consistent with the rest of the Standard Model. From an experimental point of view, we are developing technologies and huge collaborations to probe these theories. As we enter the era of high statistics, precision measurements of neutrino interactions, neutrinos might hold the key to the next generation of discoveries in particle physics.

This thesis work describes the first measurement of the $\left(\pi^{-}-\mathrm{Ar}\right)$ total hadronic cross section in the 100-1000 MeV kinetic energy range and the first measurement of the $\left(K^{+}-\mathrm{Ar}\right)$ total hadronic cross section in the 100-650 MeV kinetic energy range. These measurements were performed with the LArIAT experiment, a small ( 0.25 ton) Liquid Argon Time Projection Chamber (LArTPC) in a beam of charged particles at the Fermilab Test Beam Facility. Particle and nuclear physics have a long history of hadronic cross section measurements; what makes these measurements unique is both the target (argon) and the methodology used - the "thin slice method" - which 
takes advantage of the detection capabilities of the LArTPC technology. The combination of fine-grained tracking and excellent calorimetric information provided by the LArTPC technology enables the measurement of unprecedented details of particle interactions in argon and, in LArIAT, to measure the kinetic energy of a hadron at each step along the particle traces. A renewed interest for precision measurements of hadronic cross sections, particularly in argon, arises from the current panorama of experimental particle physics at the intensity frontier, in particular neutrino physics in LArTPCs.

Experimentally, precision measurements can be achieved only if the detector technology is able to resolve the fine details of a neutrino interaction and to record a statistically relevant number of neutrinos. With "fine details" here we mean the ability to distinguish the many products of the neutrino interaction, such as protons, pions, muons and electrons, and to measure their energy. Historically, bubble chamber neutrino detectors were the first revolution in neutrino detection: for example, the spatial resolution of Gargamelle allowed the discovery of neutrino neutral current interaction [50]. Despite the high precision of bubble chambers images, this technology is hard to scale to massive size, making statistical analyses on neutrino interactions almost impossible to perform. To make up for the small neutrino interaction cross section, neutrino experiments moved to very large size, at the expenses of spatial precision. This is the case for the detectors which discovered neutrino oscillation: both Super-Kamiokande and SNO are massive Cherenkov detectors [60]. With LArTPCs, the field is gaining again bubble-chamber like precision but at massive scales. Following the recommendations of the latest Particle Physics Project Prioritization Panel [85], the US particle physics panorama is directing a substantial effort towards the exploration of the intensity frontier through the construction of massive LArTPCs. In particular, we are seeing the development of a Short Baseline Neutrino Program (SBN) and long baseline neutrino program (DUNE), both based 
on the LArTPC detector technology. The US liquid argon program has the potential to answer many of the fundamental open questions in particle physics today, such as: is there a fourth generation neutrino? is $\mathrm{CP}$ violated in the lepton sector? are there any additional symmetries? and, can we find an indication of Grand Unified Theories?

The SBN program at Fermilab is tasked with conclusively addressing the existence of a fourth neutrino generation in the $\Delta m^{2}=\Delta m_{14}^{2} \sim[0.1-10] \mathrm{eV}^{2}$ parameter space. The SBN program entails three surface LArTPCs positioned on the Booster Neutrino Beam at different distances from the neutrino production in oder to fully exploit the $\mathrm{L} / \mathrm{E}$ dependence of the oscillation pattern: SBND (110 $\mathrm{m}$ from the decay pipe), MicroBooNE $(470 \mathrm{~m})$, and ICARUS $(600 \mathrm{~m})$. SBN will also perform an extensive program of neutrino cross section measurements, fundamental to abate systematics in the oscillation analyses in both SBN and DUNE.

DUNE has a vast neutrino and non-accelerator physics reach. Within neutrino physics, oscillation analyses in DUNE have the capability of solving the mass hierarchy and octant problem, and discovering $\mathrm{CP}$ violation in the neutrino sector. Besides its neutrino program, DUNE can open an experimental window on Grand Unified Theories (GUTs). GUTs could potentially answer fundamental questions such as the existence of non-zero neutrino masses and matter-antimatter asymmetry, explaining some "accidents" in the Standard Models, such as the exact cancellation of the proton and the electron charge. Directly probing GUTs at the unification energy scale is impossible by any foreseeable collider experiment. We then need an indirect proof such as baryon number violation, which is predicted by almost every GUT in the form of proton decay, bounded nucleon decay or $n-\bar{n}$ oscillations on long time-scales. Historically, the main technology used in these searches has been water Cherenkov detectors, with Super-Kamiokande setting all the current experimental limits on the decay lifetimes at the order of $\sim 10^{34}$ years. The DUNE far detector and its non- 
accelerator physics program is a interesting new actor on this stage. LArTPCs can in fact complement nucleon decay searches in modes where water Cherenkov detectors are less sensitive, especially $p \rightarrow K^{+} \bar{\nu}[28$.

Such a diverse physics program speaks to the versatility of the LArTPC technology. LArTPCs provide excellent electron/photon separation [77] lacking in Cherenkov detectors which can be leveraged to abate the photon background from neutral current interactions in $\nu_{e}$ searches. LArTPCs also share superb tracking capability with bubble chamber detectors, with several additional benefits. They are electronically read out and self triggered detectors; they provide full 3D-imaging with millimeter resolution, precise calorimetric reconstruction and excellent particle identification.

The amount of information a LArTPC can provide makes these detectors rather complex: a series of dedicated measurements is necessary to obtain meaningful physics results from a LArTPC. The complexity of the LArTPC technology for neutrino detection is due to several reasons. Argon is a fairly heavy element, which means that nuclear effects play an important role in the interaction topology. For example, pions are one of the main products of neutrino interactions; yet, since data on charged particle interaction in argon is scarce, neutrino event generators have big uncertainties in the re-scattering simulation of pions in argon. The amount of details in a LArTPC event can easily be parsed by human eye, but can make automatic event reconstruction rather challenging. Thus, reconstruction algorithms in LArTPC need to be tuned to recognize the different topologies of the neutrino interaction products in argon. This is particularly true for pions, since they are copiously produced of the neutrino interactions: the occurrence of a pion interaction in argon can modify the topology of the neutrino event, causing a misidentification of the neutrino interaction channel.

The LArIAT [48] experiment is performing precise cross section measurements of charged particles in argon to address this gap of knowledge. The LArIAT LArTPC sits on a beam of charged particles at the Fermilab Test Beam Facility which provides 
charge particles of the type and energy range relevant for neutrino interaction of both SBN and DUNE. The $\left(\pi^{-}-\mathrm{Ar}\right)$ hadronic cross section is a fundamental input for neutrino detectors in liquid argon, as pion interactions can modify the topology and energy reconstruction of neutrino events in the $\mathrm{GeV}$ range, where pion production is abundant. The $\left(K^{+}-\mathrm{Ar}\right)$ total hadronic cross section in LArIAT is particularly relevant for a high identification efficiency in the context of proton decay searches in DUNE in the $p \rightarrow K^{+} \bar{\nu}$ channel. In fact, the kaon-argon cross section affects the kaon topology by modifying the kaon tracking and energy reconstruction, impacting the basis for kaon identification in a LArTPC.

The cross section analyses exploit the totality of LArIAT's experimental handles; they rely on beam line detector information as well as both calorimetry and tracking in the TPC. These analyses are LArIAT's first physics results. In order to measure total hadronic cross sections on argon, several steps are necessary. The analyses start by identifying a sample of the hadron of interest in the beam line and assessing the beam line contaminations. It proceeds with tracking the hadron candidates in the TPC and measuring their kinetic energy at each point in the tracking: the fine sampling of an hadron in the TPC forms the set of "incident" hadrons. Then, the hadronic interaction point is identified and the raw cross section is calculated via the "thin slice method". Two corrections are then applied to the raw cross section - a background subtractions and a correction for detector effects - to obtain the cross section measurement, presented here.

This body of work is organized in 8 chapters. We provide a description of the theoretical framework for the measurements in Chapter 1 . Chapter 2 outlines the LArTPC detector technology, while Chapter 3 describes LArIAT experimental setup. We present the event selection for both the pion and kaon analyses, as well as the thin slice method in Chapter 4 . Chapter 5 describes the work done on the data 
and Monte Carlo samples in preparation of the cross section analyses. Chapter 6 shows the results for the $\left(\pi^{-}-\mathrm{Ar}\right)$ total hadronic cross section measurement. Chapter 7 shows the results for the $\left(K^{+}-\right.$Ar $)$total hadronic cross section measurement. We draw the final remarks on this work in Chapter 8

A series of additional studies and calibrations were necessary to perform the cross section analyses. Appendix A shows a measurement of the LArIAT LArTPC electric field using cosmic data. Appendix $\mathrm{B}$ shows an optimization of the tracking algorithms geared towards maximizing the efficiency of finding the hadronic interaction point. Appendix C shows the calorimetry calibration of the LArIAT LArTPC, which is a pivotal measurement to enable any physics analysis with TPC data. 


\section{Chapter 1}

\section{The theoretical framework}

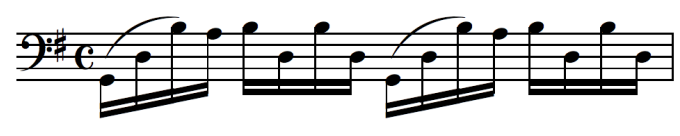

- J. S. Bach, 1720 ca. -

In this chapter, we set the $\left(\pi^{-}-\mathrm{Ar}\right)$ and $\left(K^{+}-\mathrm{Ar}\right)$ total hadronic cross section measurements into the greater theoretical and phenomenological framework. We start by briefly describing the Standard Model (Section 1.1), with particular attention to neutrinos and neutrino interactions (Section 1.2). We then describe some of the open questions in neutrino physics today and Beyond Standard Model theories (1.3) setting the stage for the measurements reported in this work (Section 1.4).

\subsection{The Standard Model}

The Standard Model (SM) of particle physics is the most accurate theoretical description of the subatomic world and, in general, one of the most precisely tested theories in the history of physics. The SM describes the strong, electromagnetic and weak interactions among elementary particles in the framework of quantum field theory, 
accounting for the unification of electromagnetic and weak interactions for energies above the vacuum expectation value (VEV) of the Higgs field. The SM does not describe gravity or general relativity.

The Standard Model is a gauge theory based on the local symmetry group

$$
G_{S M}=S U(3)_{C} \otimes S U(2)_{L} \otimes U(1)_{Y}
$$

where the subscripts $\mathrm{C}$ indicates the conserved strong charge (color), and the subscripts $\mathrm{Y}$ indicates the conserved hypercharge. If we indicated with $\mathrm{T}$ the weak isospin $\mathrm{T}$ and with $\mathrm{T}_{3}$ its third component, hypercharge can be related to the electric charge Q through the Gell-Mann-Nishijima relation:

$$
Q=\frac{Y}{2}+T_{3} .
$$

In the quantum field framework, the SM fields correspond to the irreducible representations of the $\mathrm{G}_{S M}$ symmetry group. In particular, the particles are divided in two categories, fermions and bosons, according to their spin-statistics. Described by the Fermi-Dirac statistics, fermions have half-integer spin and are sometimes called "matter-particles". Bosons or "force carriers" have integer spin, follow the BoseEinstein statistics and mediate the interaction between fermions. The fundamental fermions and their quantum numbers are listed in Tab 1.1 .

Quarks can interact via all three the fundamental forces; they are triplets of $\mathrm{SU}(3)_{C}$, that is they can exist in three different colors. If one chooses a base where $u, c$ and $t$ quarks are simultaneously eigenstates of both the strong and the weak interactions, the remaining eigenstates are usually written as $d, s$ and $b$ for the strong interaction and $d^{\prime}, s^{\prime}$ and $b^{\prime}$ for the weak interaction, because the latter ones are the result of a CKM rotation on the first ones. Charged leptons interact via the weak and the electromagnetic forces, while neutrinos only interact via the weak force. 


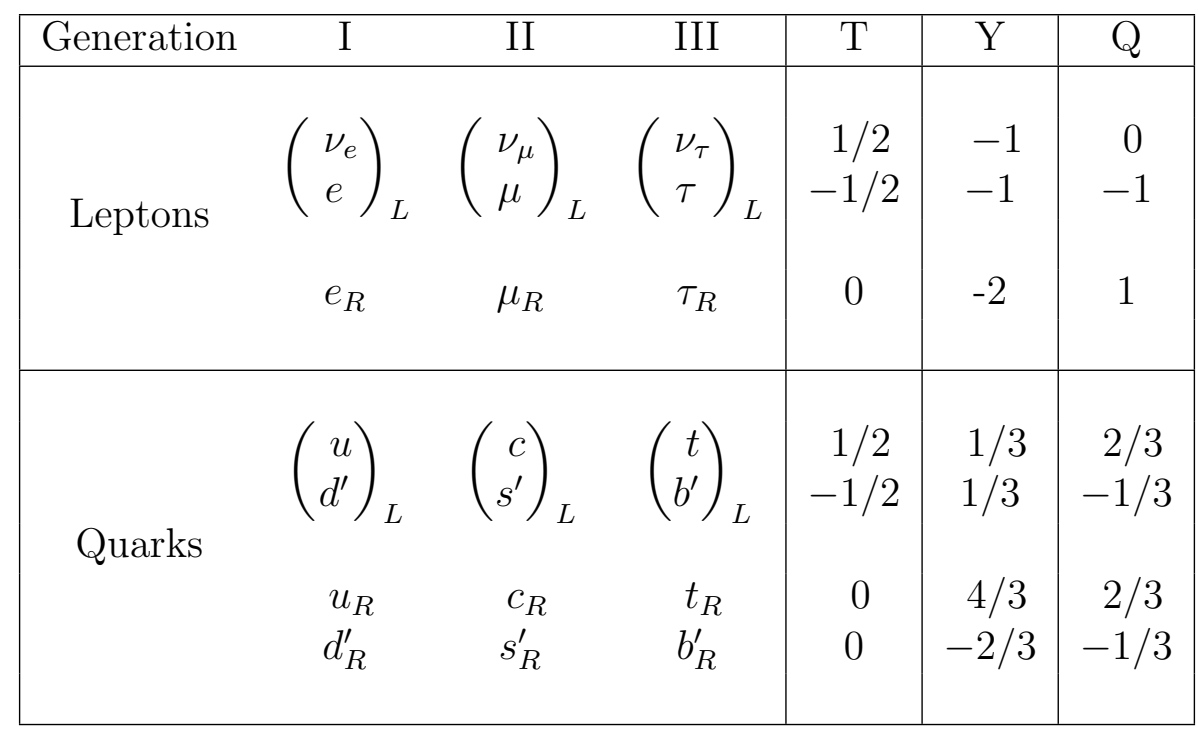

Table 1.1: SM elementary fermionic fields. The subscripts L and R indicate respectively the negative chirality (left-handed) and the positive chirality (right-handed).

The gauge group univocally determines the number of gauge bosons that carry the interaction; the gauge bosons correspond to the generators of the group: eight gluons (g) for the strong interaction, one photon $(\gamma)$ and three bosons $\left(\mathrm{W}^{ \pm}, \mathrm{Z}^{0}\right)$ for the electroweak interaction. A gauge theory by itself cannot provide a description of massive particles, but it is experimentally well known that most of the elementary particles have non-zero masses. The introduction of massive fields in the Standard Model lagrangian would make the theory not gauge invariant, resulting ill-defined. This problem is solved in the SM by the introduction of a scalar iso-doublet $\Phi(x)$, the Higgs field, which gives mass to $\mathrm{W}^{ \pm}$and $\mathrm{Z}^{0}$ gauge bosons through the electroweak symmetry breaking mechanism and to the fermions through Yukawa coupling [104]. The discovery of the Higgs boson in 2012 by the LHC experiments 9,10 marked the ultimate confirmation of a long history of successful predictions by the SM. 


\subsection{Neutrinos: tiny cracks in the Standard Model}

To our current knowledge, neutrinos are the most abundant fermion in the Universe. And yet, they are maybe the most mysterious particle in the SM: they generate theoretical puzzles and experimental challenges. In this section, we treat neutrinos within and beyond the SM and describe the make up of their interaction with matter.

\subsubsection{Neutrinos in the Standard Model}

Neutrinos can be introduced in the SM as left-handed massless Weyl spinors. The Dirac equation of motion for a free field

$$
\left(i \gamma^{\mu} \partial_{\mu}-m\right) \psi=0
$$

for a fermionic field

$$
\psi=\psi_{L}+\psi_{R}
$$

is equivalent to the equations

$$
\begin{aligned}
& i \gamma^{\mu} \partial_{\mu} \psi_{L}=m \psi_{R} \\
& i \gamma^{\mu} \partial_{\mu} \psi_{R}=m \psi_{L}
\end{aligned}
$$

for the chiral fields $\psi_{R}$ and $\psi_{L}$, whose evolution in space and time is coupled through the mass $m$. If the fermion is massless, the chiral fields decouple and the fermion can be described by a single Weyl spinor with two independent components [103]. Pauli initially rejected the description of a physical particle through a single Weyl spinor because of its implication of parity violation. In fact, since the spatial inversion operator throws $\psi_{R} \leftrightarrow \psi_{L}$, parity is conserved only if both chiral components exist at the same time. For the neutrino introduction in the SM, experiments were critical to the formulation of an accurate theoretical description. The 
constraint of parity conservation weakened after Wu's experiment in 1957 [37]. Additionally, there was no experimental indication for massive neutrinos, nor evidence of interaction via the neutrino right-handed component.

The symmetry group $S U(2)_{L} \otimes U(1)_{Y}$ is the only group relevant for neutrino interactions. The SM electroweak lagrangian is the most general renormalizable lagrangian invariant under the local symmetry group $S U(2)_{L} \otimes U(1)_{Y}$. The lagrangian couples the weak isotopic spin doublets and singlets $\left(L_{\alpha}, Q_{\alpha}, l_{\alpha}, q_{\alpha}\right)$ described in Table 1.1 with the gauge bosons $A_{a}^{\mu}(a=1,2,3)$ and $B^{\mu}$, and Higgs doublet $\Phi(x)$ :

$$
\begin{aligned}
\mathcal{L}= & i \sum_{\alpha=e, \mu, \tau} \bar{L}_{\alpha L}^{\prime} \not D L_{\alpha L}^{\prime}+i \sum_{\alpha=1,2,3} \bar{Q}_{\alpha L}^{\prime} \not D Q_{\alpha L}^{\prime} \\
& +i \sum_{\alpha=e, \mu, \tau} \bar{l}_{\alpha R}^{\prime} \not D l_{\alpha R}^{\prime}+i \sum_{\alpha=d, s, b} \bar{q}_{\alpha R}^{\prime D} \not D q_{\alpha R}^{\prime D}+i \sum_{\alpha=u, c, t} \bar{q}_{\alpha R}^{\prime U} \not D q_{\alpha R}^{\prime U} \\
& -\frac{1}{4} A_{\mu \nu} A^{\mu \nu}-\frac{1}{4} B_{\mu \nu} B^{\mu \nu} \\
& +\left(D_{\rho} \Phi\right)^{\dagger}\left(D^{\rho} \Phi\right)-\mu^{2} \Phi^{\dagger} \Phi-\lambda\left(\Phi^{\dagger} \Phi\right)^{2} \\
& -\sum_{\alpha, \beta=e, \mu, \tau}\left(Y_{\alpha \beta}^{\prime l} \bar{L}_{\alpha L}^{\prime} \Phi l_{\beta R}^{\prime}+Y_{\alpha \beta}^{\prime l *} \bar{l}_{\beta R}^{\prime} \Phi^{\dagger} L_{\alpha L}^{\prime}\right) \\
& -\sum_{\alpha=1,2,3} \sum_{\beta=d, s, b}\left(Y_{\alpha \beta}^{\prime D} \bar{Q}_{\alpha L}^{\prime} \Phi q_{\beta R}^{\prime D}+Y_{\alpha \beta}^{\prime D *} \bar{q}_{\beta R}^{\prime D} \Phi^{\dagger} Q_{\alpha L}^{\prime}\right) \\
& -\sum_{\alpha=1,2,3} \sum_{\beta=u, c, t}\left(Y_{\alpha \beta}^{\prime U} \bar{Q}_{\alpha L}^{\prime} \widetilde{\Phi} q_{\beta R}^{\prime U}+Y_{\alpha \beta}^{\prime U *} \bar{q}_{\beta R}^{\prime U} \widetilde{\Phi}^{\dagger} Q_{\alpha L}^{\prime}\right) .
\end{aligned}
$$

The first two lines of the lagrangian summarize the kinetic terms for the fermionic fields and their coupling to the gauge bosons $A_{a}^{\mu \nu}, B^{\mu \nu} 1$, The third line describes the kinetic terms and the self-coupling terms of the gauge bosons. The fourth line is the Higgs lagrangian, which results in the spontaneous symmetry breaking. The last three lines describe the Yukawa coupling between fermions and the Higgs field, origin of the fermions' mass.

1. In gauge theories the ordinary derivative $\partial_{\mu}$ is substituted with the covariant derivative $D_{\mu}$. Here $D_{\mu}=\partial_{\mu}+i g A_{\mu} \cdot I+i g^{\prime} B_{\mu} \frac{Y}{2}$, where I and $\mathrm{Y}$ are the $\mathrm{SU}(2)_{L}$ and $\mathrm{U}(1)_{Y}$ generators, respectively. 
The coupling between left-handed and right-handed field generates the mass term for fermions. The SM assumes only left-handed components for neutrinos, thus implying zero neutrino mass. Since any linear combination of massless fields results in a massless field, the flavor eigenstates are identical to the mass eigenstates in the SM.

\subsubsection{Neutrino Oscillations}

The determination of the flavor of a neutrino dynamically arises from the corresponding charged lepton associated in a charged current interaction; for example, a $\nu_{e}$ is a neutrino which produces an $e^{-}$, a $\bar{\nu}_{\mu}$ is a neutrino which produces a $\mu^{+}$, etc. The neutrino flavor eigenstates $\left|\nu_{\alpha}\right\rangle$, with $\alpha=e, \mu, \tau$, are orthogonal to each other and form a base for the weak interaction matrix.

Overwhelming experimental data show that neutrinos change flavor during their propagation [36]. This phenomenon, called "neutrino oscillations", was predicted first by Bruno Pontecorvo in 1957 [116. Neutrino oscillations are possible only if the neutrino flavor eigenstate are not identical to the mass eigenstates. Thus, the observation of neutrino oscillation results in the first evidence of physics beyond the Standard Model. A minimal extension of the SM introduces three mass eigenstates, $\left|\nu_{i}\right\rangle(i=1,2,3)$, whose mass $m_{i}$ is well defined. The unitary Pontecorvo-MakiNakagawa-Sakata matrix transforms the mass base into the flavor base as follows

$$
\left|\nu_{\alpha}\right\rangle=U_{P M N S}\left|\nu_{i}\right\rangle
$$

with 


$$
U_{P M N S}=\left[\begin{array}{ccc}
c_{12} & s_{12} & 0 \\
-s_{12} & c_{12} & 0 \\
0 & 0 & 1
\end{array}\right]\left[\begin{array}{ccc}
c_{13} & 0 & s_{13} e^{-i \delta} \\
0 & 1 & 0 \\
-s_{13} e^{-i \delta} & 0 & c_{13}
\end{array}\right]\left[\begin{array}{ccc}
1 & 0 & 0 \\
0 & c_{23} & s_{23} \\
0 & -s_{23} & c_{23}
\end{array}\right]\left[\begin{array}{ccc}
e^{i \alpha_{1}} & 0 & 0 \\
0 & e^{i \alpha_{2}} & 0 \\
0 & 0 & 1
\end{array}\right]
$$

where $c$ and $s$ stand respectively for cosine and sine of the corresponding mixing angles $\left(\theta_{12}, \theta_{23}\right.$ and $\left.\theta_{13}\right), \delta$ is the Dirac CP violation phase, $\alpha_{1}$ and $\alpha_{2}$ are the eventual Majorana CP violation phases. Experimental results on neutrino oscillations are generally reported in terms of the mixing angles and of the squared mass splitting $\Delta m_{a b}^{2}=m_{a}^{2}-m_{b}^{2}$, where $a$ and $b$ represent the mass eigenstates. A summary of the current status of experimental results extracted from [60] is given in table 1.2 .

Table 1.2: Summary of experimental results on neutrino oscillation parameters extracted from 60 .

\begin{tabular}{|r|c|c|c|}
\hline & Value & Precision & Experiment \\
\hline$\theta_{23}$ & $\sim 45^{\circ}$ & $9.0 \%$ & Super Kamiokande, MINOS, \\
$\left|\Delta m_{32}^{2}\right|$ & $2.510^{-3} \mathrm{eV}^{2}$ & $1.8 \%$ & NO $\nu$ a, MACRO, Super Kamiokande \\
\hline$\theta_{12}$ & $34^{\circ}$ & $5.8 \%$ & SNO, Gallex, \\
$\left|\Delta m_{12}^{2}\right|$ & $7.410^{-5} \mathrm{eV}^{2}$ & $2.8 \%$ & SAGE, KamLAND \\
\hline$\theta_{13}$ & $9^{\circ}$ & $4.7 \%$ & Daya Bay, \\
$\left|\Delta m_{32}^{2}\right|$ & $2.510^{-3} \mathrm{eV}^{2}$ & $1.8 \%$ & RENO \\
\hline
\end{tabular}

\subsubsection{Make up of Neutrino Interactions}

All neutrino experiments involving the detection of single neutrinos are concerned with neutrino interactions (and neutrino cross sections) on nuclei. Given the invisible nature of the neutrino, characterizing the products of its interaction is the only method to a) assess the neutrino presence, b) detect its flavor in case of a charged current interaction and c) eventually reconstruct its energy.

Historically, neutrino interactions with the nucleus in the $\mathrm{GeV}$ region are divided into three categories whose contributions change as a function of increasing neutrino 
energy: quasi-elastic (QE), resonant (RES), and deep inelastic (DIS) scattering. All current and near future oscillation experiments on neutrino beams live in the 0.1-10 $\mathrm{GeV}$ transition region, which encompasses the energy where the QE neutrino-nucleus interaction transitions into RES and then into DIS. For scattering off free nucleons, neutrino and antineutrino QE charged current scattering refers to the process $\nu_{l} n \rightarrow l^{-} p$ and $\bar{\nu}_{l} p \rightarrow l^{+} n$ where a charged lepton and single nucleon are ejected in the elastic interaction. Resonant scattering refers to an inelastic collision producing a nucleon excited state $\left(\Delta, N^{*}\right)$ - the resonance - which then quickly decays, most often to a nucleon and single-pion final state. DIS refers to the head-on collision between the neutrino and a parton inside the nucleon, producing hadronization and subsequent abundant production of mesons and nucleons. In addition to such interactions between the neutrino and a single component of the nucleus, neutrinos can also interact with the nucleus as a whole, albeit more rarely, a well documented process called coherent meson production [68]; the signature of such process is the production of a distinctly forward-scattered single meson final state, most often a pion. This simple picture of neutrino interactions works rather well for scattering off of light nuclear targets, such as the $\mathrm{H}_{2}$ and $\mathrm{D}_{2}$ of bubble chamber experiments [54], but the complexity of the nuclear structure for heavier nuclei such as argon complicates this model.

As we will discuss in Chapter 2, the properties of argon make it a good candidate for an interacting medium in neutrino experiments; in particular the density of its interaction centers increases the yield of neutrino interactions and allows for relatively compact detectors. Though, the choice of a relatively heavy nuclear target comes at the cost of enhancing nuclear effects which modify the kinematic and final state of the neutrino interaction products.

Nuclear effects can potentially affect measured neutrino event rates, final state particle emission, neutrino energy reconstruction, and the neutrino/antineutrino ra- 
tios, carrying deep implications for oscillation experiments. Even in the case of "simple" QE scattering, intra-nuclear hadron rescattering and correlation effects between the target nucleons can cause the ejection of additional nucleons in the final state, modifying the final state kinematics and topology. In the case of resonant and DIS scattering, the hadronic interactions of meson and nucleons produced in the decay of the resonance or during hadronization complicate this picture even more. A large source of uncertainty in modeling nuclear effects in neutrino interactions comes from meson interactions (and re-interactions) in the nucleus, e.g., pion re-scattering, charge exchange, and absorption.

There has been a renewed interest in neutrino cross section measurements in recent years, along with a lively discussion on the data reporting; the historical method of reporting the neutrino cross section as a function of the neutrino energy or momentum transferred shakes under the weight of its dependency on the chosen nuclear model. On one hand, correcting for nuclear effects in neutrino interactions can introduce unwanted sources of uncertainty and model dependency, especially due to the mis-modeling of the meson interactions. On the other, avoiding this correction makes a comparison between neutrino interactions on different target nuclei extremely difficult.

Data on neutrino scattering off many different nuclei are available for both charged current (CC) and neutral current (NC) channels, as summarized in [54]. A summary of the results of QE, resonant and DIS scattering for neutrinos and antineutrinos from accelerators on different target is reported in Figure 1.1, where the (NUANCE) 7] event generator prediction is shown in comparison with experimental data. 

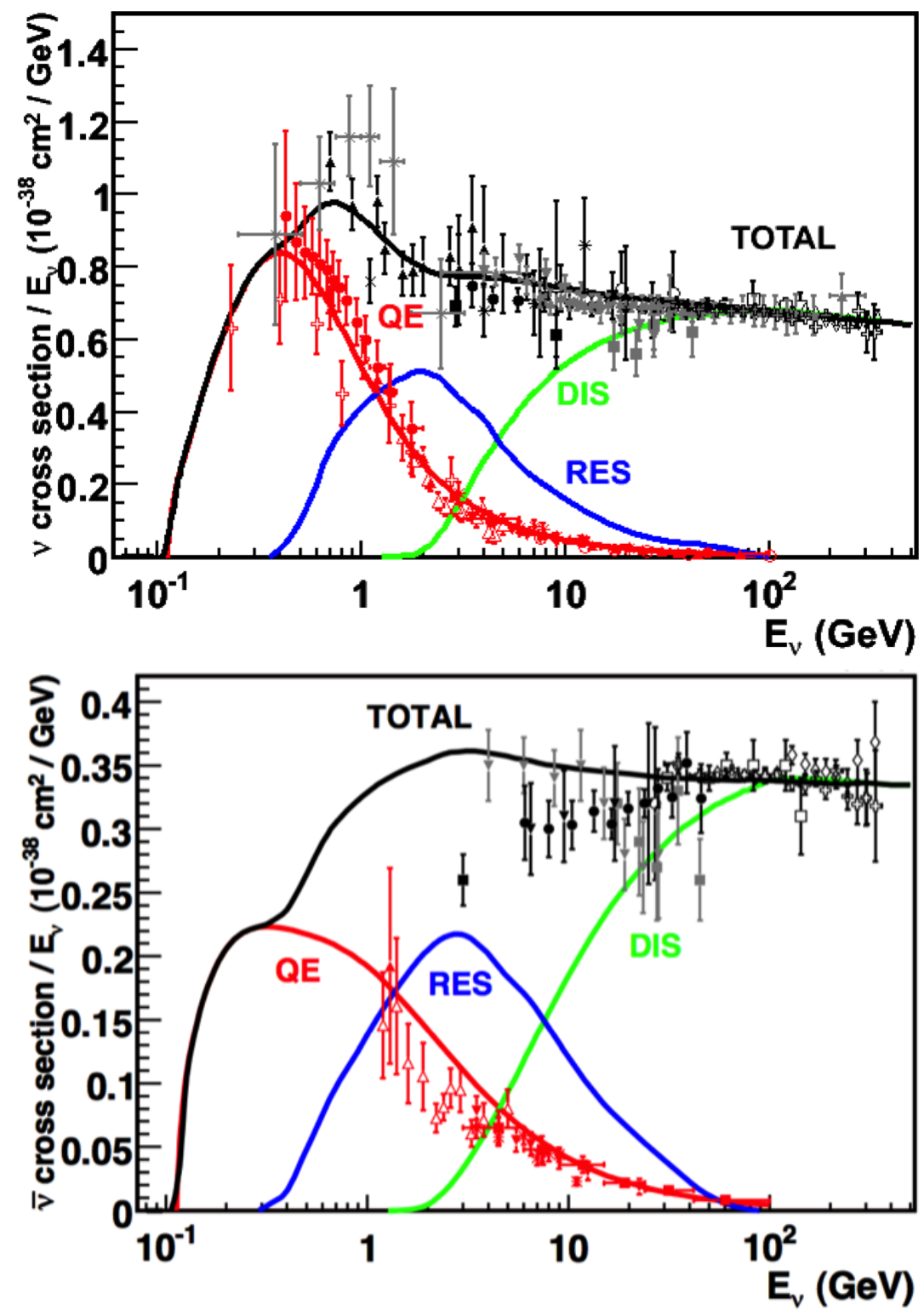

Figure 1.1: Total neutrino (top) and antineutrino (bottom) CC cross sections per nucleon divided by neutrino energy as a function of energy as reported in [54]. Predictions for the total (black), the QE (red), resonant (blue) and DIS (green) are provided by the NUANCE generator. The quasi-elastic scattering data and predictions have been averaged over neutron and proton targets (isoscalar target). 


\subsection{Beyond the Standard Model}

The discovery of neutrino oscillation and its implication of non-zero neutrino mass mark the beginning of a new, exciting era in neutrino physics: the era of physics Beyond the Standard Model (BSM) at the intensity frontier. We are currently searching for new, deeper theories that can accommodate neutrinos with tiny but non-zero masses, while remaining consistent with the rest of the Standard Model.

\subsubsection{Open Questions in Neutrino Physics}

On one hand, the last three decades of experiments in neutrino oscillations brought spectacular advancements in the understanding of the oscillations pattern, measuring the neutrino mixing angles and mass splitting with a precision better than $10 \%$. On the other, they opened the field for a series of questions needing experimental answers.

Sterile neutrinos. Hints to the existence of at least one additional neutrino, in the form of various anomalies, have been puzzling physicists almost from the beginning of neutrino oscillation searches. Originally designed to look for evidence of neutrino oscillation, the Liquid Scintillator Neutrino Detector (LSND) 31 provided a first conflicting result with the Standard Model expectation of only three neutrinos. A second conflicting result has also been provided by the MiniBooNE experiment 23]. The LSND and MiniBooNE $\nu_{e}$ and $\bar{\nu}_{e}$ appearance results, known as the "LSND and MiniBooNE anomalies" 15, 16, 32, may be interpreted under the assumption of a new right-handed neutrino. The additional neutrino needs to be "sterile" i.e, needs not to couple with the electroweak force carriers, in order to meet the constraint imposed by the measurement of the width of the $\mathrm{Z}$ boson [87]. The new sterile neutrino would mainly be composed of a heavy neutrino $\nu_{4}$ with mass $m_{4}$ such that $m_{1}, m_{2}, m_{3} \ll m_{4}$ and $\Delta m^{2}=\Delta m_{14}^{2} \sim[0.1-10] \mathrm{eV}^{2}$. The introduction of sterile neutrinos is an appealing line of thinking, since this renormalizable generalization of the SM has the 
potential to impact long standing questions in high energy physics and cosmology: light sterile neutrinos are candidates for dark matter particles and there are ideas that the theory could be adjusted to explain the baryon asymmetry of the Universe via leptogenesis [100].

CP Violation In Lepton Sector. The measurement of non-zero value for the oscillation parameter $\theta_{13}$ allows the exploration of low-energy $\mathrm{CP}$ violation in the lepton sector at neutrino long baseline oscillation experiments, enabling the possibility to measure the Dirac CP-violating phase $\delta$. Exciting theoretical results tie $\delta$ directly to the generation of the baryon asymmetry of the Universe at the Grand Unified Theory scale. According to the theoretical model described in [84], for example, leptogenesis can be achieved if $\left|\sin \theta_{13} \sin \delta\right|>0.11$, i.e. $\sin \delta>0.7$.

The asymmetry in the oscillation probability of neutrinos and antineutrinos is the observable sensitive to the Dirac CP-violating phase $\delta$ leveraged in neutrino oscillation experiments. Using the parameterization of the PMNS matrix shown in Equation 1.9, the difference between the probability of $\nu_{e} \rightarrow \nu_{\mu}$ oscillation and the probability of $\bar{\nu}_{e} \rightarrow \bar{\nu}_{\mu}$ oscillation can be parametrized as follows [19],

$$
P_{\nu_{e} \rightarrow \nu_{\mu}}-P_{\bar{\nu}_{e} \rightarrow \bar{\nu}_{\mu}}=J \cos \left( \pm \delta-\frac{\Delta_{31} L}{2}\right) \sin \left(\frac{\Delta_{21} L}{2}\right) \sin \left(\frac{\Delta_{31} L}{2}\right)
$$

where

$$
J=\cos \theta_{13} \sin 2 \theta_{13} \sin 2 \theta_{12} \sin 2 \theta_{23}
$$

is the Jarlskog invariant [107], $L$ the neutrino baseline, i.e. the distance between the neutrino production and detection points, and $\Delta_{a b}$ a factor proportional to the sign and magnitude of the mass splitting. From these equations, it is clear how the relatively large value of $\theta_{13}$ is a happy accident necessary not to completely suppress the sensitivity to $\mathrm{CP}$ violation. The equations also show how the sensitivity to $\delta$ is tied to the measurement of the least precisely measured mixing angle, $\theta_{23}$ (via the 
$\sin 2 \theta_{23}$ term) and to another unknown quantity, the neutrino "mass hierarchy" (via the $\Delta_{a b}$ terms). The precise determination of $\theta_{23}$ is often referred as to "the octant problem". Current experimental results [12,59] are consistent with $\theta_{23}=45^{\circ}$, which would imply maximal mixing between $\nu_{\mu}-\nu_{\tau}$, hinting to an intriguing new symmetry. Therefore, a precise measurement of $\theta_{23}$ is of great interest for theoretical models of quark-lepton universality [53, 102, 117], whose quark and lepton mixing matrices are proportional to the deviation of $\theta_{23}$ from $45^{\circ}$.

Neutrino mass hierarchy. The "mass hierarchy" problem refers to the unknown ordering of the value of absolute mass of the neutrino mass eigenstates. Current oscillation experiments are sensitive only to the magnitude of the mass splitting, and not directly to its sign. In a framework where the lightest neutrino mass (arbitrarily) corresponds to the first eigenstate $m_{1}$, it is unknown whether $m_{2}-m_{1}<m_{3}-m_{1}$ (Normal Hierarchy) or $m_{2}-m_{1}>m_{3}-m_{1}$ (Inverted Hierarchy). The mass hierarchy affects not only the sensitivity to $\mathrm{CP}$ violation searches in long baseline oscillation experiments, but also the sensitivity to determine whether neutrinos are Majorana particles in neutrinoless double beta decay experiments.

Majorana or Dirac? Evidence of neutrino oscillations demands the introduction of a mechanism which can give mass to the neutrinos. This mechanism should possibly also explain why neutrino masses are at least six orders of magnitude lower than the electron mass (the second lightest SM fermion). In a description of neutrinos as Dirac 4-component spinors, the neutrino field acquires mass via the Higgs mechanism as any other fermion of the SM. In this case, the neutrino mass is given by $m_{a}=\frac{y_{a}^{\nu} v}{\sqrt{2}}$, where $v$ is the Higgs VEV and $y_{a}^{\nu}$ is the Yukawa coupling between the Higgs and the neutrino. The smallness of neutrino masses can only be pinned on a tiny Yukawa coupling which is not justified by the theory.

In 1937, Majorana demonstrated that the introduction of a two-component spinor is sufficient to describe a massive fermion [110]. The Dirac equations of motion for the 
chiral fields (equations 1.5 and 1.6 hold true in the case of a two-component spinor under the assumption that the chiral components $\psi_{R}$ and $\psi_{L}$ are correlated through the charge conjugation matrix $\mathcal{C}, \psi_{R}=\mathcal{C} \bar{\psi}_{L}$. Therefore the theory is applicable only to neutral fermions. Neutrinos are the only neutral elementary particles in the SM - the only possible Majorana particle candidate. This theory constructs a neutrino Majorana mass term $\mathcal{L}_{5}$ of the following form in the Higgs unitary gauge

$$
\mathcal{L}_{5}=\frac{1}{2} \frac{g v^{2}}{\mathcal{M}} \nu_{L}^{T} \mathcal{C}^{\dagger} \nu_{L}
$$

where $g$ is the coupling coefficient, $v$ the Higgs VEV, and $\mathcal{M}$ a constant with the dimension of the mass proportional to the scale of new physics. The $\mathcal{L}_{5}$ term would introduce a non-renormalizable term in the lagrangian, since it has dimensions of energy to the fifth power. This is not allowed in the SM lagrangian; however, the existence of such terms is plausible if we consider the SM as an effective theory at low energy, manifestation of the symmetry breaking of a more general theory at higher energy, e.g. a Grand Unified Theory (GUT), and not the definitive theory. The mass term in Equation 1.12 implies the neutrino mass to be $m=\frac{g v^{2}}{\mathcal{M}}$. The coupling coefficient can be of the order of any other fermion's coupling coefficient, since the smallness of neutrino masses is achieved by the large value of the new physics mass scale alone. This vanilla formulation is the conceptual basis for many flavors of seesaw mechanism [122], which we will not discuss here in any detail. However, it is fascinating how the puzzle of the neutrino mass hints to the existence of a deeper and more complete theory.

From a kinematic point of view, Dirac and Majorana neutrinos satisfy the same energy-momentum dispersion relationship. Thus, it is impossible to discern the neutrino nature through kinematic effects such as neutrino oscillations. Neutrinoless double beta decay $(0 \nu \beta \beta)$ searches are the most promising way to understand the 
nature of the neutrino and are therefore a subject of great theoretical and experimental interest. Observation of the lepton number violating process $0 \nu \beta \beta$ would imply neutrinos have a Majorana component. Depending on the mass hierarchy, the theory also predicts $0 \nu \beta \beta$ exclusion regions and confirmation of the sole Dirac component for neutrinos 81 .

\subsubsection{Towards a more fundamental theory: GUTs}

Despite its highly predictive power, a number of conceptual issues arise in the SM which disfavor it to be a good candidate for a fundamental theory.

The SM does not include a suitable dark matter candidate and a mechanism that accounts for the baryon asymmetry of the universe. Additionally, up to a total of 25 parameters remain seemingly arbitrary and need to be fitted to data: 3 gauge couplings, 9 charged fermion masses, 3 mixing angles and one CP phase in the CKM matrix, the Higgs mass and quartic coupling, $\theta_{Q C D}, 3$ neutrino mixing angles, 1 Dirac phase and, eventually, 2 Majorana phases.

From a group theory perspective, the SM has a rather complex group structure, where a gauge group is formed with the direct product of other three groups as shown in Equation 1.1. Drawing a parallel with the electroweak symmetry breaking mechanism, where the $S U(2)_{L} \otimes U(1)_{Y}$ is recovered from $U(1)_{E M}$, an interesting line of simplification for the SM group structure would be to devise a similar mechanism where $S U(3)_{C} \otimes S U(2)_{L} \otimes U(1)_{Y}$ is recovered from an hypothetical larger group. Just as the electroweak unification becomes evident at energies higher than the Higgs VEV, a direct manifestation of Grand Unification Theories (GUTs) would occur at even higher energies.

As the smallness of neutrino masses suggests the existence of a higher mass scale, another, even stronger, hint for Grand Unification comes from the slope of running 
of the coupling constants. The coupling constants for the electromagnetic, weak and strong interactions in the SM vary as a function of the interaction energy as shown in Figure 1.2; they do not exactly meet under the current experimental constraints, but their trend is interesting enough to push for the construction of theories where perfect unification is achieved through the addition of new particles.

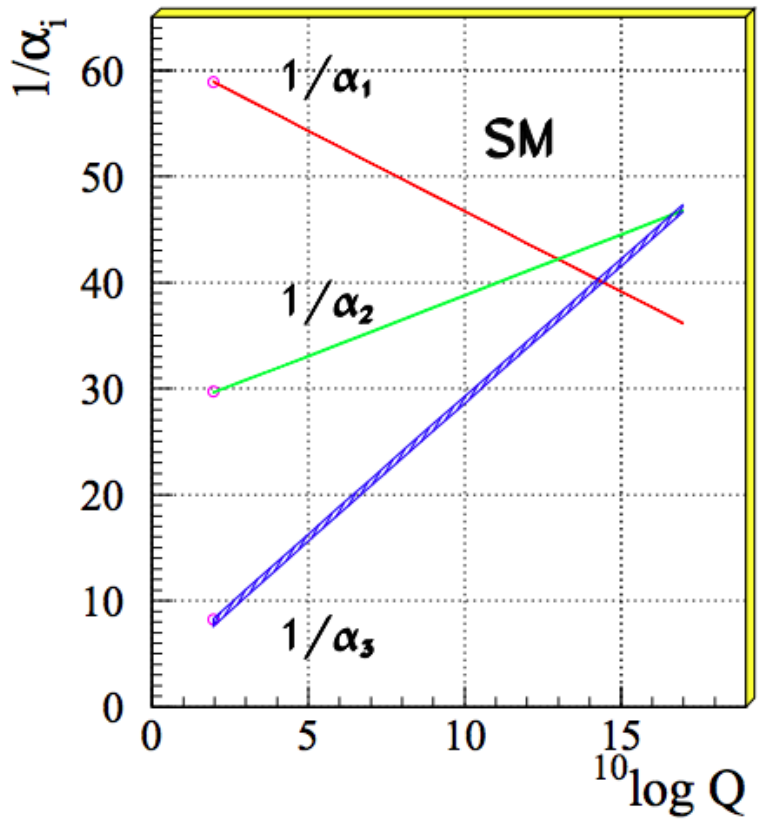

Figure 1.2: Evolution of the inverse of the three coupling constants in the Standard Model as a function of the momentum transferred, [109].

$\mathbf{S U}(5)$. The smallest simple group containing $S U(3)_{C} \otimes S U(2)_{L} \otimes U(1)_{Y}$ is $\mathrm{SU}(5)$, as shown first by Georgi and Glashow in [52]. Quarks and leptons in this group fit the $\overline{5}$ and 10 representations. The representation for left-handed fermions are the following

$$
\begin{aligned}
\overline{5} & =\left(\nu_{e}, e^{-}\right)_{L}+\bar{d}_{L} \\
10 & =e_{L}^{+}+\bar{u}_{L}+(u, d)_{L},
\end{aligned}
$$


while the boson structure gains a new couple of super heavy bosons $(\mathrm{X}, \mathrm{Y})$

$$
24=\underbrace{(8,1)}_{\text {gluons }}+\underbrace{(1,3)+(1,1)}_{\mathrm{W}^{ \pm}, \mathrm{Z}, \gamma}+\underbrace{(3,2)+(\overline{3}, 2)}_{\mathrm{X}, \mathrm{Y} \text { bosons }} .
$$

Nice features such as charge quantization and the identity between the positron and proton charge value come directly from the group structure. The new super heavy bosons are colored and form a weak doublet. They are the mediator of the interaction that turns quarks into leptons, leading to predict the existence of processes that violate baryon number, such as $p \rightarrow \pi^{0}+e^{+}$(see Figure 1.9, right). The prediction for proton decay lifetime, $\tau_{p} \sim \frac{M_{X}^{4}}{m_{p}^{5}} \sim 10^{30 \pm 1.5}$ years, is unfortunately experimentally disproven by IMB and Super-Kamiokande [13,98].

SO(10). More complicated group structures, such as $\mathrm{SO}(10)$ are still viable candidates for GUT. $\mathrm{SO}(10)$ includes the same type of $X$ and $Y$ bosons as $\mathrm{SU}(5)$. Right-handed massive neutrinos are embedded in the construction of the irreducible representation of $\mathrm{SO}(10)$. Different patterns of $\mathrm{SO}(10)$ symmetry breaking to recover the SM are possible and lead to different predictions for the proton decay lifetime; some of these predictions are not excluded by the experiments $[42]$.

SUSY GUTs. Supersymmetry theories allow for another family of GUTs. In SUSY, every fundamental particle in the SM has a "superpartner", identical in each quantum number except for the spin-statistics: the fermion supersymmetric partners are bosons and vice versa. Collider experiments (mainly LHC) constrain the mass of the supersymmetric partners to be very heavy. The SU(5), SU(10) groups with a SUSY twist are the basic groups for SUSY GUTs. From the phenomenology point of view, SUSY models tend to push the proton decay life time higher by a factor of four, they solve the "hierarchy problem", and they also predict new channels for the proton decay. In particular they predict the presence of kaons in the final product, with a dominant mode of $p \rightarrow K^{+} \bar{\nu}$. Predictions of the proton decay lifetime depend 
on the chosen SUSY model; again, some of the predictions are not excluded by the experiments $56,69,90$.

\subsection{Motivations for Hadronic Cross Sections in Ar- gon}

Critical challenges await the next decade of high energy physics at the intensity frontier. Following the recommendation of the latest Particle Physics Project Prioritization Panel [85], the US is dedicating substantial resources to the development of a short- and long- baseline neutrino program to address many of open questions in neutrino physics today. This program pivots on the Liquid Argon Time Projection Chamber (LArTPC) detector technology which will be described in Chapter 2 .

The main goals of these research programs include:

- the assessment of the existence of sterile neutrinos via the study of accelerator neutrinos on a short baseline (SBN),

- the determination of the sign of $\Delta m_{13}^{2}\left(\right.$ or $\left.\Delta m_{23}^{2}\right)$, i.e., the neutrino mass hierarchy via the study of accelerator neutrinos on a long baseline (DUNE),

- the determination of the octant, i.e., whether $\theta_{23}$ is maximal, via the study of accelerator neutrinos on a long baseline (DUNE),

- the determination of the status of CP symmetry in the lepton sector, via the study of accelerator neutrinos on a long baseline (DUNE),

- the search for observables predicted by GUTs, such as proton decay via the study of non accelerator physics in massive underground detectors (DUNE). 


\subsubsection{Pion-Argon Total Hadronic Cross Section}

This section outlines the importance of the pion-argon total hadronic cross section in the context of the current and upcoming liquid argon neutrino experiments, SBN and DUNE. We describe the signal signature and historic measurements of pion-nucleus cross sections, as well as the implementation of these cross sections in the current version of the simulation package used by LArIAT.

\section{$\pi^{-}$Ar Cross Section in the Context of Neutrino Searches}

As outlined in 1.2.3, neutrino experiments use the products of neutrino interactions to identify the energy and flavor of the incoming neutrino. Pions are a common product of neutrino interactions, especially in resonant scattering, DIS and coherent pion production. For neutrino experiments in argon, there are two main reasons why understanding pion hadronic interactions with argon is important: to model the behavior of the pion inside the target nucleus and to model the behavior of the pion during its propagation inside the detector medium.

Assumptions on the nuclear modeling and on the interaction of hadrons inside the nucleus performed at the level of the neutrino event generator bridge the measurement of the products of a neutrino interaction to the reconstruction of the neutrino energy and flavor. Thus, understanding pion hadronic interactions with the nucleus is particularly important to model correctly resonant, DIS and coherent pion production in neutrino interactions. For example, in case of resonant scattering,

$$
\nu_{l}+N \rightarrow l+\Delta / N^{*} \rightarrow l+\pi+N^{\prime},
$$

the $\Delta$ and $N^{*}$ and excited states will decay hadronically in matters of $\sim 10^{-24} \mathrm{~s}$ inside the nucleus, producing pions which which will have many chances to re-interact as they exit the target medium. The decay modes for the lower mass $\Delta(1232)$ and 
$N^{*}(1440)$ are listed in table 1.3 .

\begin{tabular}{|l|l|l|}
\hline Resonance & Decay Mode & Lifetime (s) \\
\hline & & \\
& $\Delta^{++}($uuu $) \rightarrow p \pi^{+}$ & \\
& $\Delta^{+}($uud $) \rightarrow n \pi^{+}$ & \\
$\Delta(1232) 3 / 2^{+}$ & $\Delta^{+}($uud $) \rightarrow p \pi^{0}$ & $\sim 5.6 \times 10^{-24}$ \\
& $\Delta^{0}\left(\right.$ udd $\rightarrow n \pi^{0}$ & \\
& $\Delta^{0}($ udd $) \rightarrow p \pi^{-}$ & \\
& $\Delta^{-}($ddd $) \rightarrow n \pi^{-}$ & \\
\hline & & \\
$N^{*}(1440) 1 / 2^{+}$ & $N^{*} \rightarrow N \pi$ & $\sim 2.2 \times 10^{-24}$ \\
& $N^{*} \rightarrow N \pi \pi$ & \\
\hline
\end{tabular}

Table 1.3: Main decay modes of the lightest Delta resonance and Nucleon excited state.

The key elements of neutrino event generators for resonance and DIS events are the nuclear model and the hadron treatment (both production and transportation). We illustrate here the conceptual basis of the GENIE Neutrino Generator [30] as an example, since GENIE is one the most popular event generators for liquid argon experiments. For example, the nuclear model used by GENIE for all processes is a Relativistic Fermi Gas (RFG) model modified to incorporate nucleon-nucleon correlations [17]. This means that the initial momentum and binding energy of the struck nucleon is determined by assuming nucleons inside the nucleus are quasi-free, acting independently in the mean field of the nucleus. For A > 20 such as argon, the 2-parameter Woods-Saxon shell model for density function is used. The GENIE module INTRANUKE [35] is used to simulate final-state interactions (FSI) which model hadron re-interactions inside the nucleus. This module places the outgoing particles in the nucleus and propagates them using the "hA model". In the INTRANUKE hA model, hadrons can undergo at most one FSI per event. When possible, external hadron-nucleus scattering data are used to tune INTRANUKE. Since no data are available for argon, GENIE uses an interpolation of data from heavier and lighter 
nuclei for the pion-argon cross section leading to resultant uncertainties in the INTRANUKE module of the order of $10-20 \%$.

Once the pion has left the target nucleus, the pion-argon hadronic cross section also plays an important role in the pion transportation inside the argon medium: processes such as pion absorption or pion charge exchange can greatly modify the topology of a neutrino interaction in the detector and lead to significant modifications in the event classification. Being able to reconstruct the details of pions inside the detector is an imperative for modern liquid argon neutrino experiments to achieve the design resolution for their key physics measurements.

\section{$\pi^{-}$Ar Hadronic Interaction: Signal Signatures}

Strong hadronic interaction models [46, 120] predict the pion interaction processes with argon in the $[100-1200] \mathrm{MeV}$ energy range. The total hadronic $\pi^{-}$-Ar interaction cross section is defined as the one related to the single process driven only by the strong interaction which is dominant in the considered energy range. In measuring the "total" cross section, we include both the elastic and reaction channels, regardless of the final state,

$$
\sigma_{\text {Tot }}=\sigma_{\text {Elastic }}+\sigma_{\text {Reaction }}
$$

the reaction channel is further characterized by several exclusive channels with defined topologies,

$$
\sigma_{\text {Reaction }}=\sigma_{\text {Inelastic }}+\sigma_{\text {abs }}+\sigma_{\text {chex }}+\sigma_{\text {mprod }}
$$

A summary of the pion final states in order of pion multiplicity for the reaction channel is given in table 1.4. Figure 1.3 shows examples of the topologies due to the pion-argon hadronic interaction as they appear in the LArIAT data: elastic and inelastic scattering, pion absorption with emission of protons, charge exchange and production of pions. 

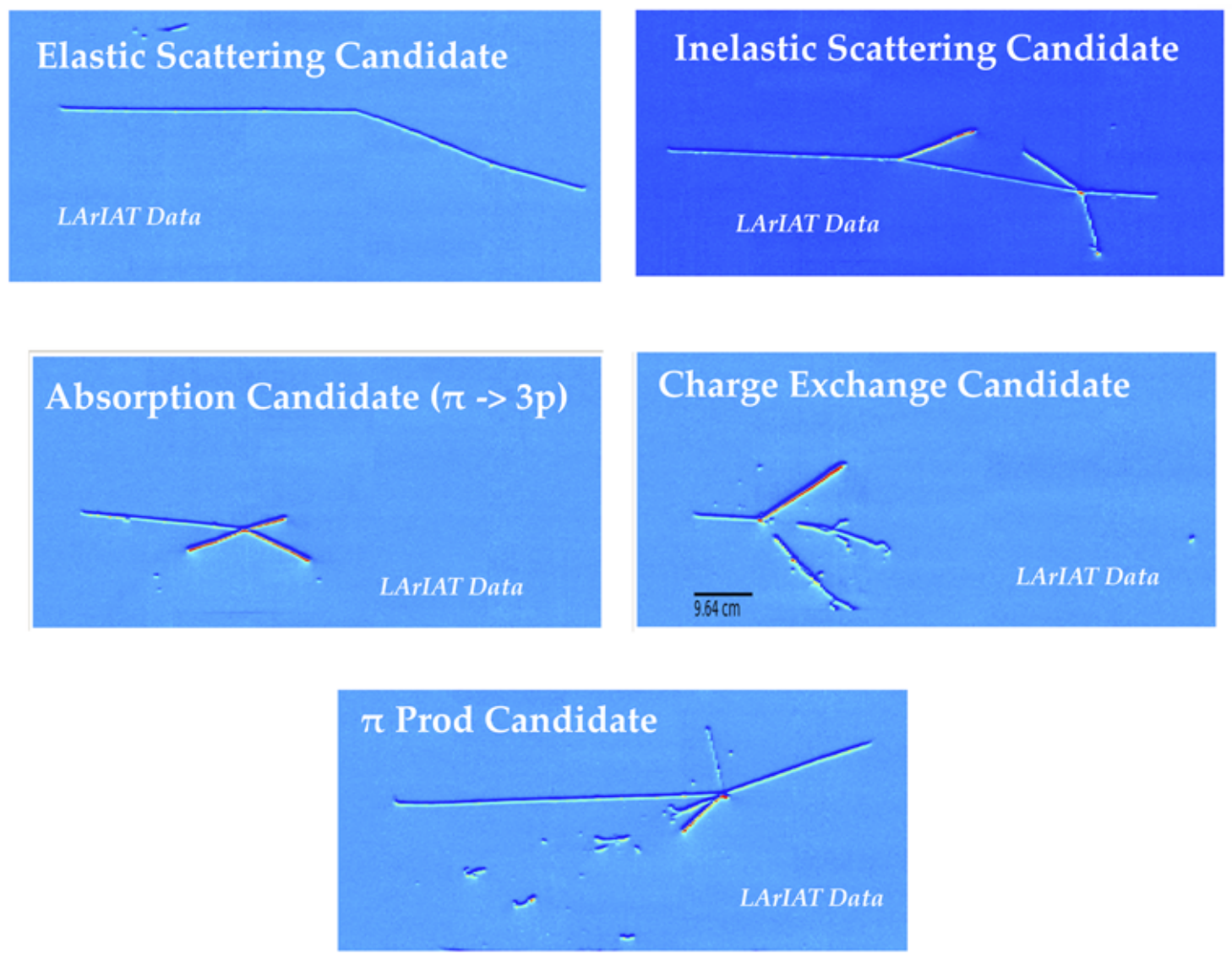

Figure 1.3: Pion-Argon interaction topologies due to elastic scattering, inelastic scattering, pion absorption with emission of protons, charge exchange and production of pions as seen in LArIAT data (induction plane only). 
Pion capture and pion decay at rest dominate the cross section under $100 \mathrm{MeV}$. We define pion capture as the process determining the formation of a pionic atom and the subsequent pion's end of life. Stopping negative pions can form pionic argon, where the negative pion plays the role of an orbital electron. Since the pion mass is two orders of magnitude greater than the electron mass, the spatial wave form of the pion will overlap more with the nucleus compared to the electron case. After the electromagnetic formation of the pionic atom, the pion will get quickly absorbed by the nucleus, which is put in an excited state. The nucleus then de-excites with the emission of low energy nucleons and photons. Pion capture is dominant compared to pion decay, the other important process for very low energy pions. The decay of a pion is governed by the weak force; the pion decay life time is $\tau_{\pi}=2.6 \times 10^{-8} \mathrm{~s}$ and the main decay mode is $\pi^{-} \rightarrow \mu^{-}+\bar{\nu}_{\mu}$ (BR 99.98\%). Since pion capture can be considered an electromagnetic process and pion decay is a weak process, this energy region is purposely excluded from the hadronic cross section measurement.

\section{Previous measurements: Lighter and Heavier Nuclei}

Many experiments with pion beams have studied the hadronic interaction of pions on light and heavy materials, such as $\mathrm{He}, \mathrm{Li}, \mathrm{C}, \mathrm{Fe}, \mathrm{Pb}[22]$. However, data on argon are rare: the total differential hadronic cross section has never been measured before on argon. Simulation packages such as Geant4 base their pion transportation for argon on data from lighter and heavier nuclei: the goal of LArIAT's dedicated measurement on argon is to bridge this gap in data, thus reducing the uncertainties related to pion interactions in argon in both neutrino event generators and in simulation packages of pion transportation.

The shape of the pion-nucleus interaction cross section in the energy range considered shows the distinct features indicating the presence of a resonance. In fact, the mean free path of a pion of kinetic energy between 100 and $400 \mathrm{MeV}$ is much shorter 


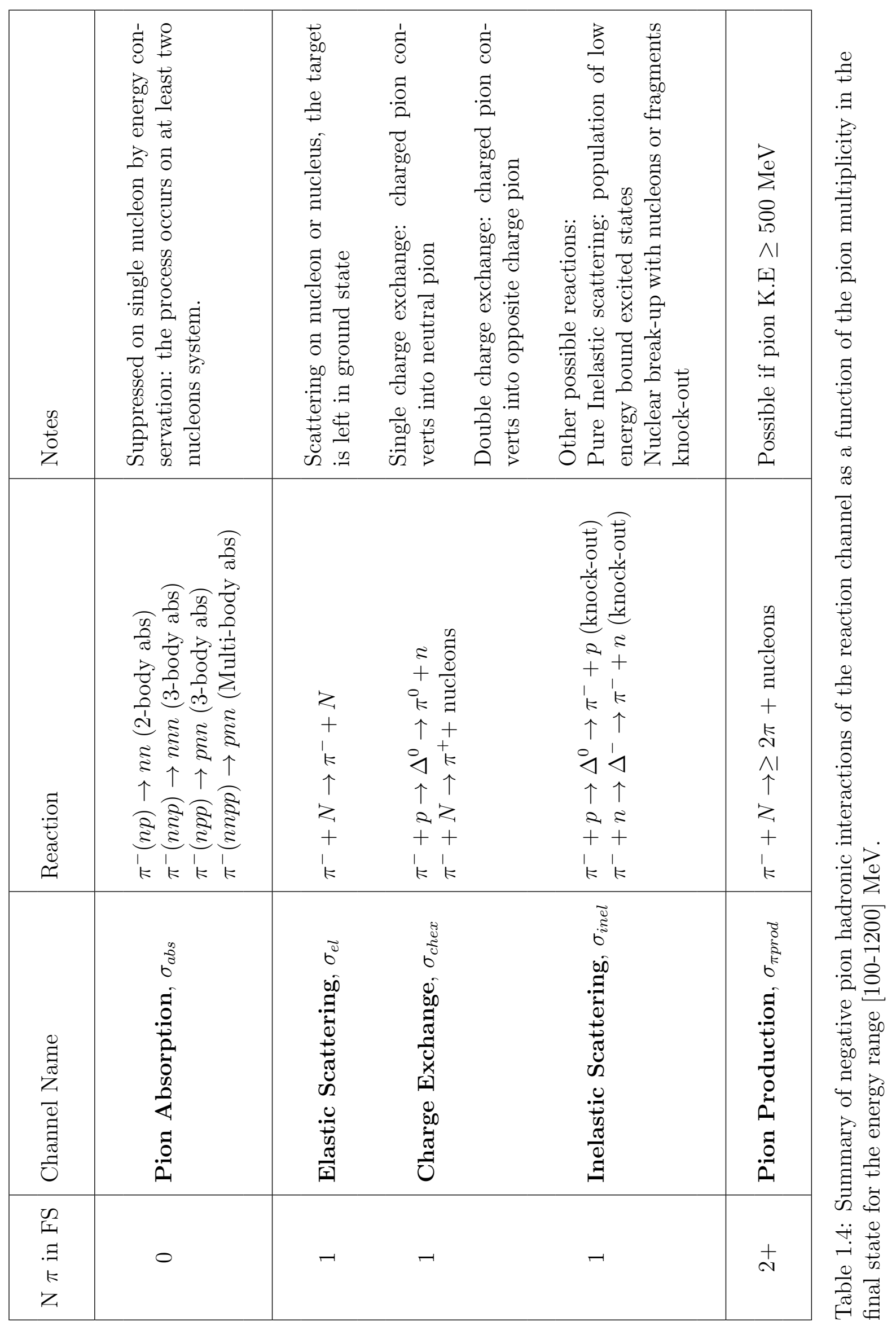


than the average distance between nucleons (which is of the order of $1 \mathrm{fm}$ ). Therefore, the pion interacts with surface nucleons. A $\Delta$ resonance is often produced in the interaction, which subsequently decays inside the nucleus. Experimental results on several nuclei as reported in [22] are shown in Figure 1.4 ; it is interesting to notice here how the shape of the $\Delta$ resonance becomes less pronounced as a function of the mass number of the target nucleus. Pion interactions with heavier nuclei also shift the peak of the resonance to lower energy; this effect is due to kinematic considerations and to the difference in propagation of the $\Delta$ inside the nucleus. Multiple scattering effects modify the resonance width, which is larger than the natural-decay width. As an example of a fairly well studied target, Figure 1.5 reports the negative pion cross section on Carbon for the elastic and reaction ${ }^{2}$ channels, and their sum 47].

\section{Negative Pion Interaction Cross Section in Simulation Packages}

LArIAT uses Geant4 as the default simulation package. In particular, pion (and kaon) transportation is achieved through the Geant4 FTFP_BERT physics list. In this physics list, Geant4 uses the Bertini cascade model [41] to simulate the products of the pion-nucleus interaction as well as secondary hadronic re-interactions inside the target nucleus (intra-nuclear cascade). The target nucleus is represented as a continuous gas where the nuclear potential follows concentrical shells whose depths approximate the Woods-Saxon shape. The CERN-HERA compilations [88, 89] of hadron-nucleon interaction data is the database used for the decision making process after the cascade is invoked. The cross section model determines if the pion interacts, the eventual type of interaction and the interaction multiplicity. For hadron projectiles with energy less than $20 \mathrm{GeV}$, Geant4 reports the uncertainty on the cross section model to be about the size of the error bars on the data used, or about $10 \%$, increasing to $20-30 \%$ in energy regions where data are sparse.

2. This paper calls "inelastic interaction" what we refer as to "reaction channel". 


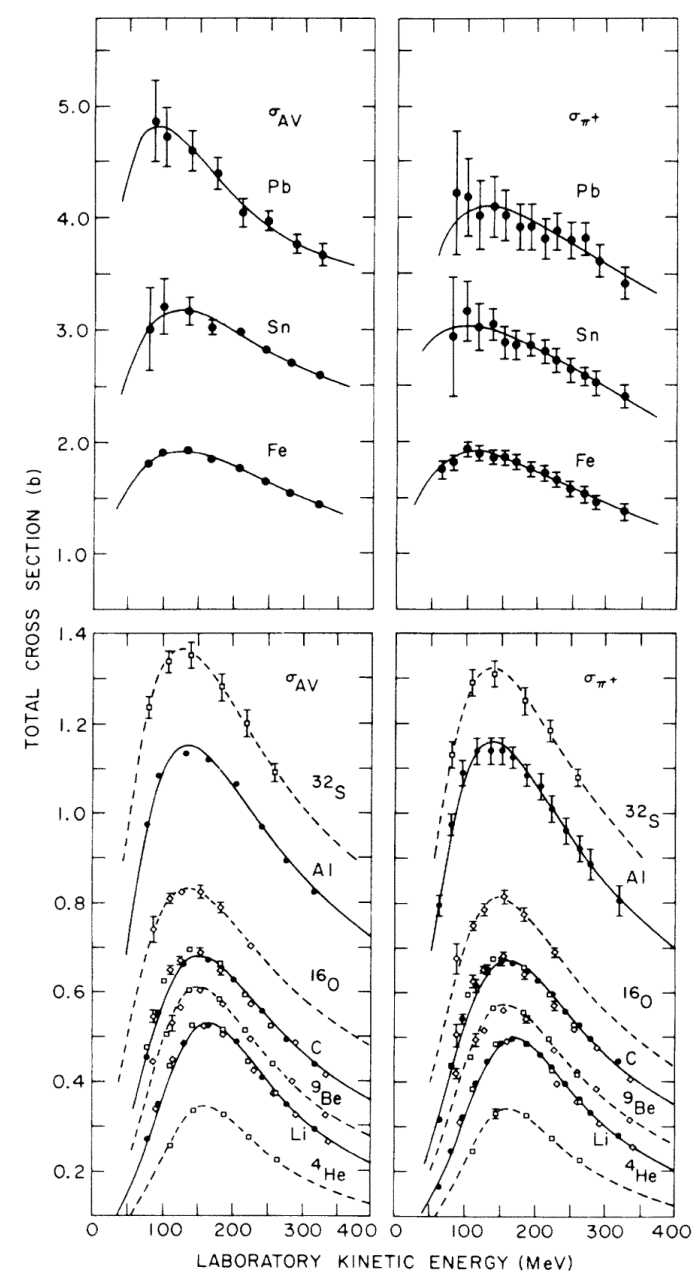

Figure 1.4: Pion-nucleus total cross sections: $\sigma_{\pi^{+}}$for positive pions (right) and $\sigma_{A V}$ (left) for the average between positive and negative pions $\sigma_{A V}=\frac{\sigma_{\pi^{+}+\sigma_{\pi^{-}}}}{2}$ in the $\Delta$ resonance region. The error bars include estimates of systematic uncertainties. The curves are the results of fits to the data assuming a Breit-Wigner shape. This summary plot is reported in [22] and uses data from [24,38]. 


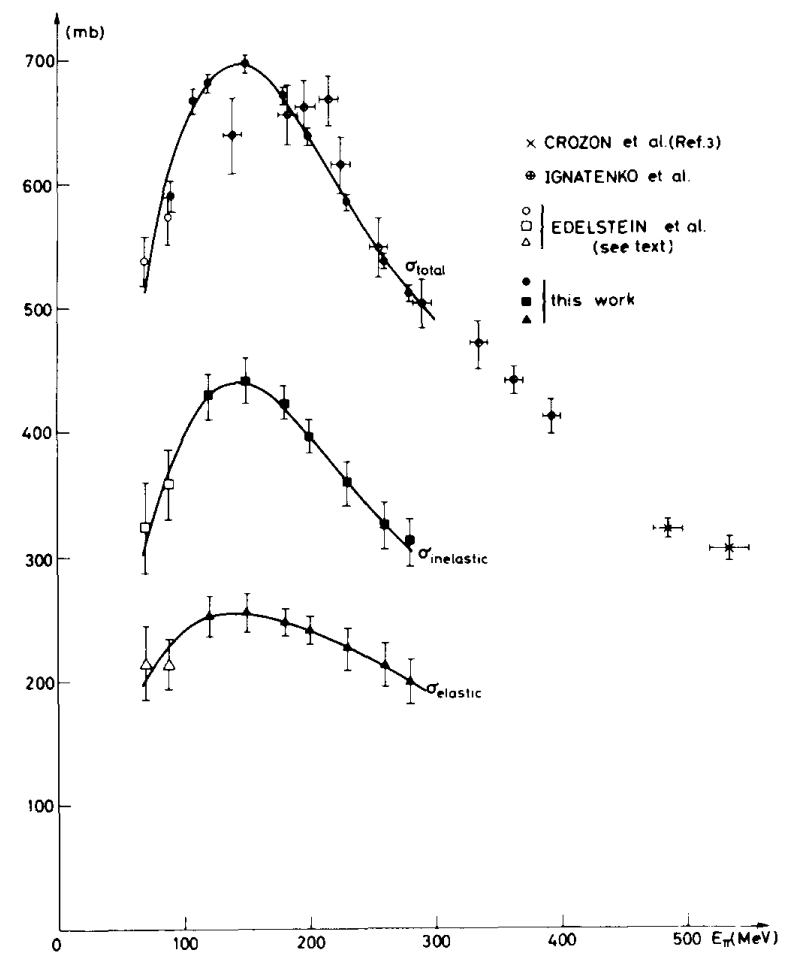

Figure 1.5: Negative pion nucleus total, elastic and reaction cross sections on ${ }^{12} \mathrm{C}$ as from [47].

The relevance of the GENIE generator for neutrino physics and its basic working principles have been outlined earlier in this section. Given GENIE's modularity, information on hadron-nucleus interactions can be extracted from the INTRANUKE module and directly compared against the Geant4 predictions. The work in [113 reviews the current status of negative and positive pion simulation in Geant4 and GENIE for ${ }^{12} \mathrm{C},{ }^{56} \mathrm{Fe}$, and ${ }^{40} \mathrm{Ca}$. From that work, we report the results for ${ }^{12} \mathrm{C}$ in Figure 1.6 as it allows a direct comparison between Geant4, GENIE and pion rescattering data. Geant4 predictions for $\pi^{-}$on Carbon are in good agreement with data over the entire spectrum, while GENIE predictions seem to show some features at around $500 \mathrm{MeV}$ and $900 \mathrm{MeV}$, maybe due to higher resonances in the hA model. From the same work, we also report the negative pion cross section on ${ }^{40} \mathrm{Ca}$ in Figure 1.7. since this is the nuclear medium closest to argon. The predictions from both Geant4 and GENIE agree with data in the high energy region; the Geant4 and GENIE 


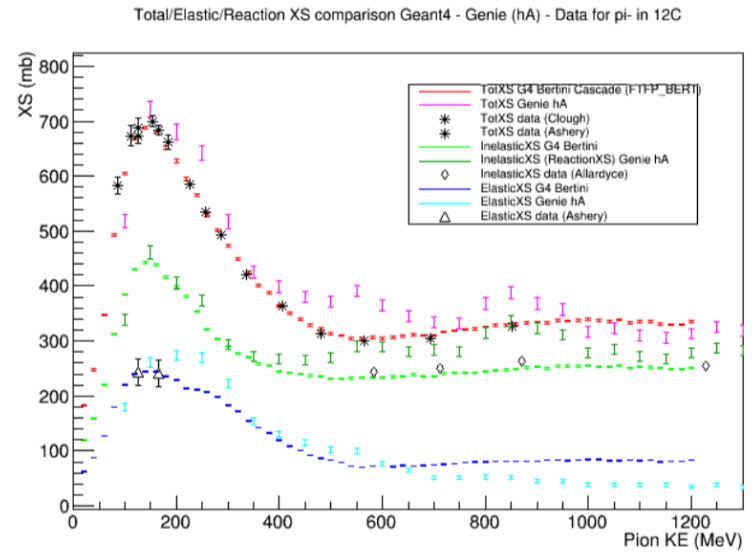

Figure 1.6: Total, elastic and reaction cross section for $\pi^{-}$on ${ }^{12} \mathrm{C}$. Comparison between results from Geant4 simulation (Bertini cascade model), GENIE simulation (hA model), and experimental data [24, 27, 39, 119].

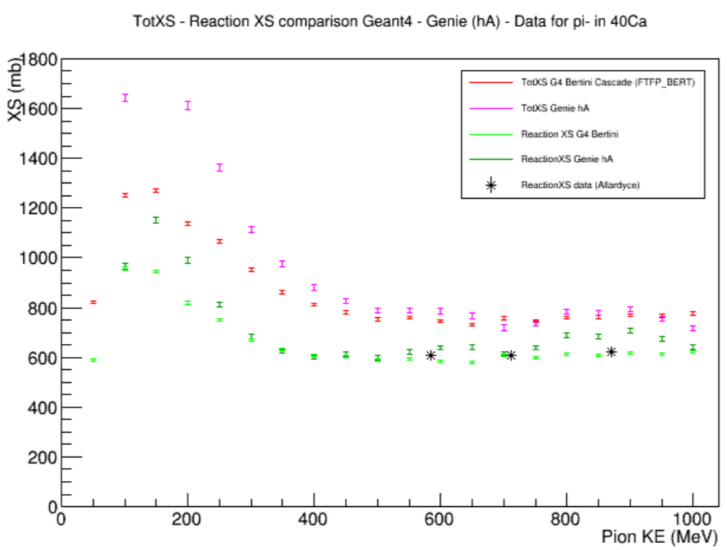

Figure 1.7: Total, elastic and reaction cross section for $\pi^{-}$on ${ }^{40} \mathrm{Ca}$. Comparison between results from Geant4 simulation (Bertini cascade model), GENIE simulation (hA model), and experimental data 27$]$.

predictions diverge in the resonance region, where data are not available. These few examples highlight how cross section data for the specific nucleus considered in the neutrino experiments is fundamental to inform the Monte Carlo simulation.

For the LArIAT simulation of the MC sample used in the $\pi^{-}$argon total hadronic cross section measurement we use the Geant4 Bertini Cascade model, whose predictions for the total, elastic and reaction hadronic cross sections are shown in Figure 1.8 . 


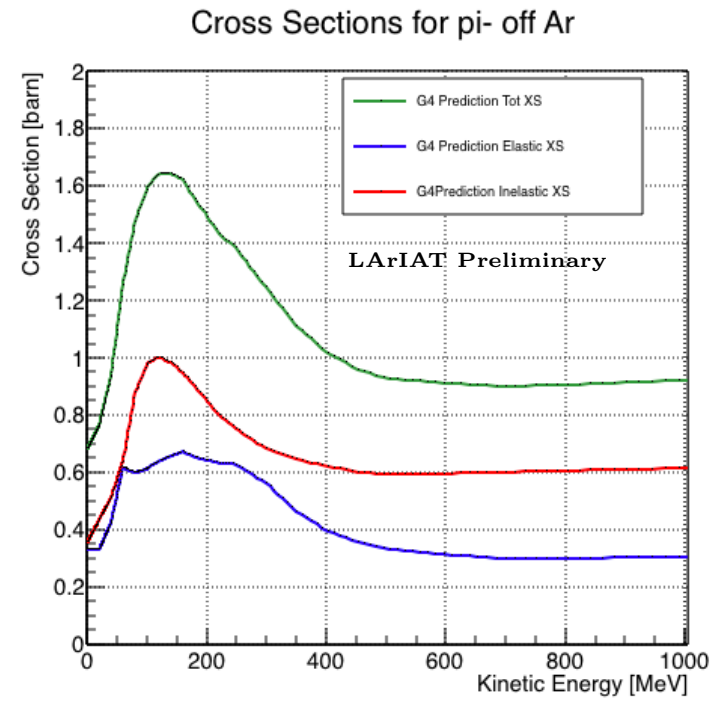

Figure 1.8: Total, elastic and reaction hadronic cross section for $\pi^{-}$-argon implemented in Geant4 10.01.p3 using FTBF_BERT physics list. 


\subsubsection{Kaon-Argon Total Hadronic Cross Section}

This section outlines the importance of the kaon-argon total hadronic cross section. We start by discussing the measurement in the context of nucleon decay searches. We then describe the signal signature and historical measurements of kaon-nucleus cross section, as well as the implementation of this cross section in the current version of the simulation package used by LArIAT.

\section{$\mathrm{K}^{+}$Ar Cross section in the Context of Nucleon Decay Searches}

Baryon number is accidentally conserved in the Standard Model. Even though no baryon number violation has been experimentally observed thus far, no underlying symmetry in line with the Noether paradigm [112] explains its conservation. As shown in section 1.3.2, almost all Grand Unified Theories predict at some level baryon number violation in the form of nucleon decay on long time-scales. Given the impossibility to reach grand unification energy scales with collider experiments (Energy Scale $>10^{15} \mathrm{GeV}$ ), an indirect proof of GUTs is needed. The experimental observation of nucleon decay may be the only viable way to explore these theories.

In case of nucleon decay discovery, the dominant decay mode may uncover additional information about the GUT type. Supersymmetric GUTs 25, 82, prefer the presence of kaons in the products of the decay, e.g. $p \rightarrow K^{+} \bar{\nu}$ (see fig 1.9 , left). Gauge mediated GUTs, in which new gauge bosons are introduced that allow for the transformation of quarks into leptons, and vice versa, prefer the mode $p \rightarrow e^{+} \pi^{0}$ (see fig 1.9 , right).

LArIAT's tiny active volume makes it impossible for the experiment to place competitive limits on nucleon decay searches. However, LArIAT provides excellent data to characterize kaons in liquid argon for the "LAr golden mode", $p \rightarrow K^{+} \bar{\nu}$. The result of these studies will affect future proton decay searches in LArTPCs. Previous work has been done to assess the potential identification efficiency for different decay 

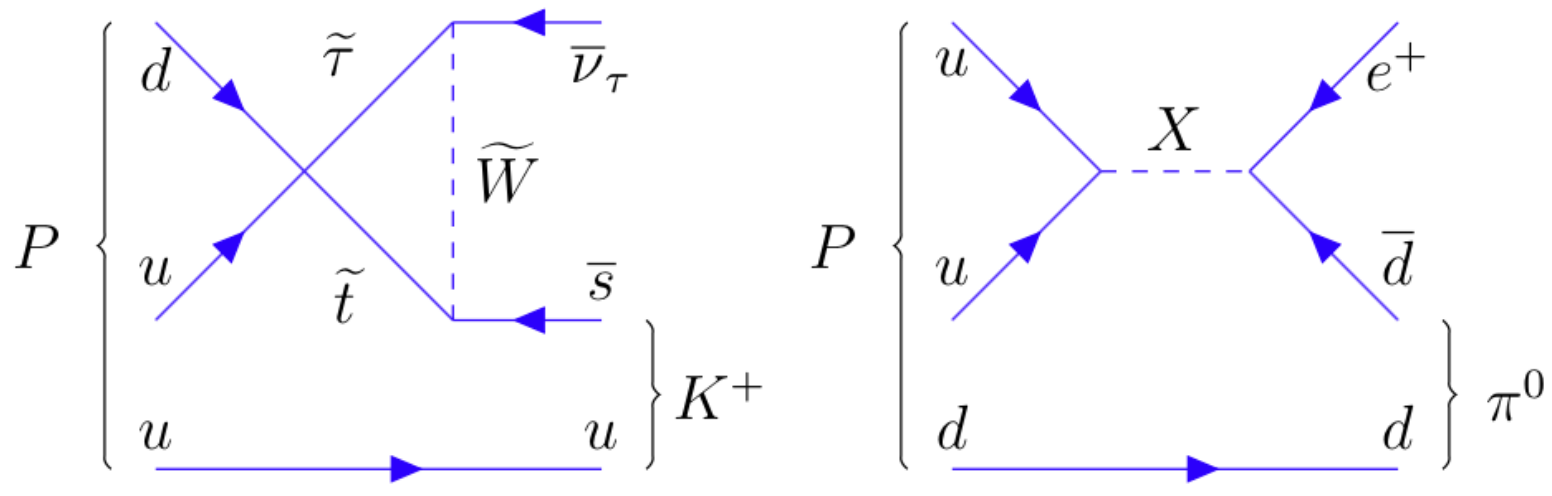

Figure 1.9: Feynman diagrams for proton decay "golden modes": $p \rightarrow K^{+} \bar{\nu}$ for supersymmetric GUTs on the left and $p \rightarrow e^{+} \pi^{0}$ for gauge-mediated GUTs on the right.

modes in a LArTPC [18], but, as the time of this writing, no study of kaon selection efficiency in LArTPCs has been performed on data. The $\mathrm{K}^{+}-\mathrm{Ar}$ interaction cross section has never been measured before and can affect the possibility of detecting and measuring kaons when produced in a proton decay event. Kaon interactions with argon can distort the kaon energy spectrum as well as change the topology of single kaon events. In a LArTPC, non-interacting kaons appear as straight tracks with a high ionization depositions at the end (Bragg peak). The topology of interacting kaons can be quite different. In case of elastic scattering, a distinct kink will be present in the track. In case of inelastic scattering the Bragg peak will not be present and additional tracks will populate the event. Performing the total hadronic $\mathrm{K}^{+}-\mathrm{Ar}$ cross section measurement on data serves the double purpose of identifying the rate of "unusual" topologies (kinks and additional tracks) and of developing tools for kaon tracking in LAr.

\section{$K^{+}$Ar Hadronic Interaction: Signal Signatures}

The interaction of a mildly relativistic charged kaon with an argon nucleus is determined largely by the strong force. The total hadronic $\mathrm{K}^{+}-\mathrm{Ar}$ interaction cross section 
is defined as the one related to the single (hadronic) process driven only by the strong interaction. In this case, "total" indicates all strong interactions regardless of the final state. This condition purposefully includes both elastic and inelastic (reaction) channels. Indeed, the total cross section section can be then decomposed into

$$
\sigma_{\text {Tot }}=\sigma_{\text {Elastic }}+\sigma_{\text {Reaction }}
$$

Figure 1.10 shows examples of the topologies due to the kaon-argon hadronic interaction as they appear in the LArIAT data: elastic and inelastic scattering.

\section{Elastic Scattering Candidate}

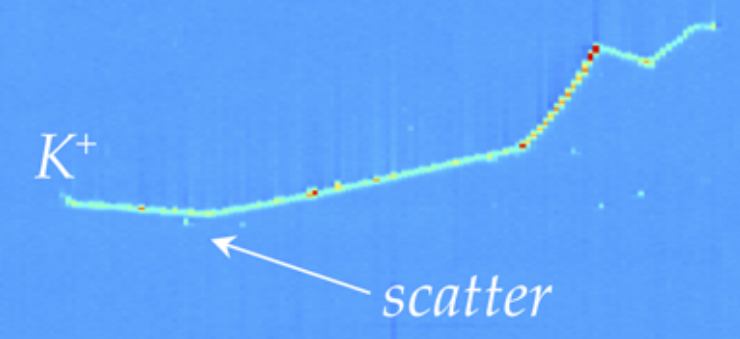

LArIAT Data

\section{Inelastic Scattering Candidate}

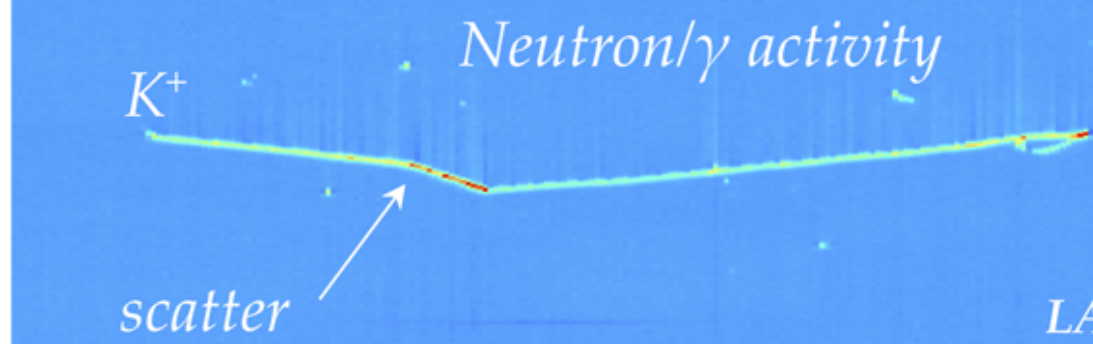

\section{LArIAT Data}

Figure 1.10: Kaon-Argon interaction topologies due to elastic scattering and inelastic scattering as seen in LArIAT data (collection plane only).

For the LArIAT cross section analysis, the kaons considered span a momentum inside the TPC from $100 \mathrm{MeV} / \mathrm{c}$ to $800 \mathrm{MeV} / \mathrm{c}$. In this energy range, the relevant 
K-Nucleon interactions are according to 97 :

$$
\begin{aligned}
K^{+}+N & \rightarrow K^{+}+N \text { (elastic) } \\
K^{+}+n & \rightarrow K^{0}+p \text { (elastic) } \\
K^{+}+N & \rightarrow K+N+\pi \text { (inelastic) } \\
K^{+}+N & \rightarrow K^{*}+N \text { (inelastic). }
\end{aligned}
$$

\section{Previous Measurements: Lighter and Heavier Nuclei}

In general, measurements on kaon cross sections are extremely scarce. The measurement of the kaon interaction cross section would bring the additional benefit of reducing the uncertainties associated with hadron interaction models adopted in MC simulations for argon targets, beneficial for both proton decay studies and kaon production from neutrino interaction studies, where the uncertainties for final state interaction models are big [40].

Figure 1.11 shows a 1997 measurement on several elements as performed by Friedmann et al. [4]. As a reference, this paper measures a $\sigma_{\text {Tot }}$ for $\mathrm{Si}$ of $366.5 \pm 4.8 \mathrm{mb}$ and a $\sigma_{T o t}$ for $\mathrm{Ca}$ of $494.6 \pm 7.7 \mathrm{mb}$ at $488 \mathrm{MeV} / \mathrm{c}$. The cross section for argon is expected to lie in between these two measurements. Additional data on the kaon cross section are provided by Bugg et al. [43]. Bugg performs a measurement of the total $\mathrm{K}^{+}$and $\mathrm{K}^{-}$cross sections on protons and deuterons over the range of 0.6-2.65 $\mathrm{GeV} / \mathrm{c}$, as well as a measurement of the total $\mathrm{K}^{+}$and $\mathrm{K}^{-}$cross sections on carbon for a number of momenta; the results of this paper on carbon are reported in Figure 1.12 . 


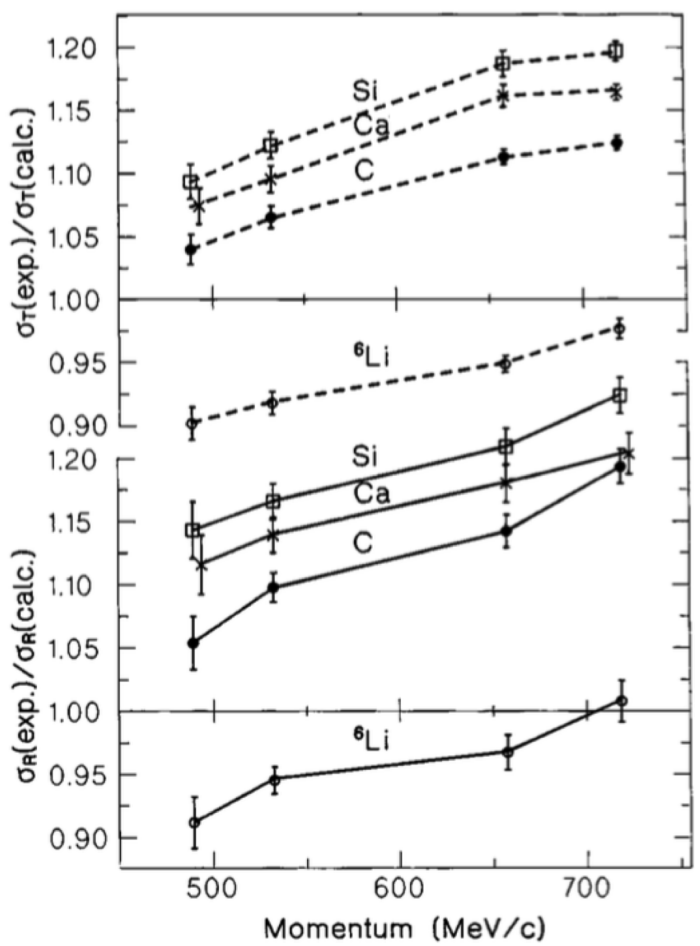

Figure 1.11: Ratios between experimental and calculated cross sections as from 44. Top: Total cross sections.

Bottom: reaction cross sections.

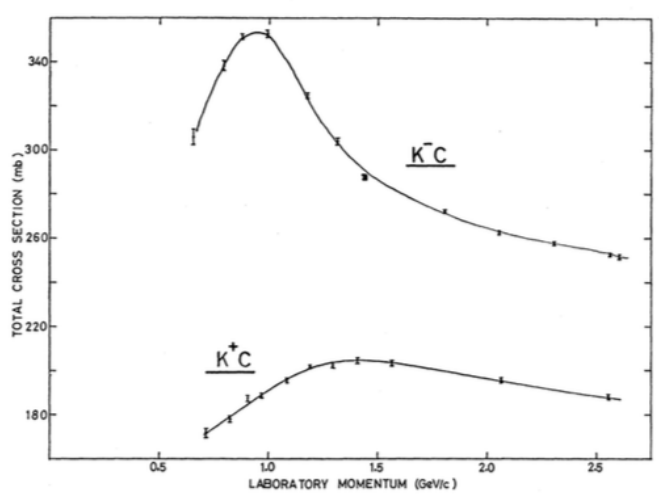

Figure 1.12: Total $\mathrm{K}^{+}$and $\mathrm{K}^{-}$cross sections on carbon as from 43 . 


\section{Kaon Interaction Cross Section for thin target in Geant4}

Since the kaon cross section in argon has never been measured before, simulation packages tune kaon transportation in argon by extrapolation from lighter and heavier nuclei. LArIAT uses the Geant4 suite for particle transportation. Since kaon data on carbon are available, we used it as a metric to evaluate the Geant4 prediction performances. Figure 1.13 shows the total hadronic cross section for carbon implemented in Geant4 10.01.p3 overlaid with the Bugg and Friedman data. Unfortunately, version 10.01.p3 3 of Geant4, which is the version used for the simulation in this work, does not reproduce the data for carbon closely. On one hand, this evidence makes us even more wary when using the Monte Carlo in simulating the kaon-argon interactions. On the other, it further highlights the importance of the kaon measurement. For the LArIAT simulation of the MC sample used in the $\mathrm{K}^{+}$argon total hadronic cross section measurement we use the Geant4 Bertini Cascade model, whose predictions for the total, elastic and reaction hadronic cross sections are show in Figure 1.14 .

3. It should be noted that the latest Geant4 version, 10.03.p3, uses a different parametrization for the kaon cross section and retrieves a better agreement with data. 


\section{$\left(\mathrm{K}^{+}, \mathrm{C}\right)$ Total Hadronic Cross Section}

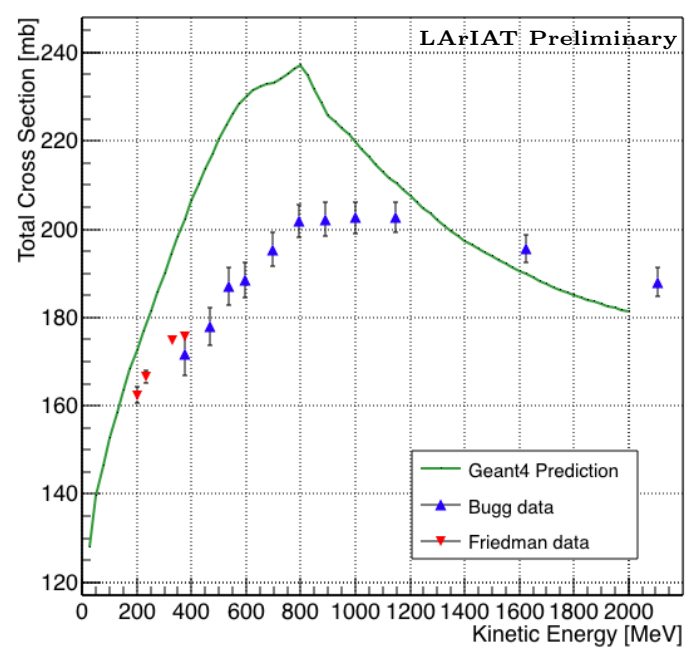

Figure 1.13: Total hadronic cross section for carbon implemented in
$\left(\mathrm{K}^{+}, \mathrm{Ar}\right)$ True Cross Section

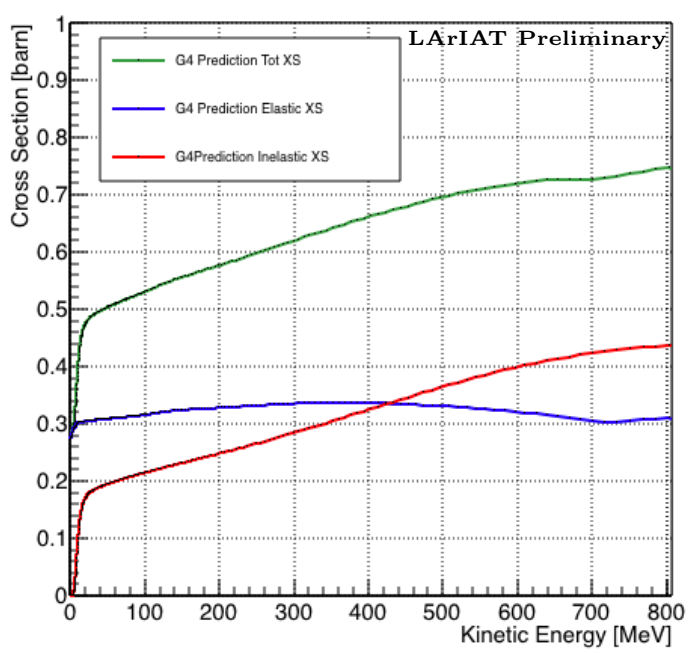

Figure 1.14: Total, elastic and reaction hadronic cross section for $K^{+}$-argon Geant4 10.01.p3 with overlaid with the implemented in Geant4 10.01.p3. Bugg and Frideman data. 


\section{Chapter 2}

\section{Liquid Argon Detectors at the Intensity Frontier}

"Don't you know, honey, Ain't nobody ever gonna love you, the way I try to do?"

- Janis Joplin, 1971 -

In the next few years, LArTPCs will be the tools to answer some of the burning questions in neutrino physics today. This chapter illustrates the operational principles of this detector technology, as well as the scope of the key detectors in the US liquid argon program - SBN, DUNE and LArIAT.

\subsection{The Liquid Argon Time Projection Chamber Technology}

In this section, we outline an extremely brief history of Time Projection Chambers as particle detectors, focusing on their incarnation as argon detectors for neutrino physics. We further describe the working principles of Liquid Argon Time Projection 
Chambers, leading to the description of the event reconstruction in LArTPC.

\subsubsection{TPCs, Neutrinos \& Argon}

David Nygren designed the first Time Projection Chamber (TPC) in the late 1970s [114] for the PEP-4 experiment, a detector apt to study electron-positron collisions at the PEP storage ring at the SLAC National Accelerator Laboratory. From the original design in the seventies - a cylindrical chamber filled with methane gas - the TPC detector concept has seen many incarnations, the employment of several different active media and a variety of different particle physics applications, including, but not limited to the study of electron/positron storage rings (e.g. PEP4, TOPAZ, ALEPH and DELPHI), heavy ions collisions in fixed target and collider experiments (e.g. EOS/HISS and ALICE ), dark matter (ArDM), rare decays and capture (e.g. TRIUMP, MuCap), neutrino detectors and nucleon decay (ICARUS, SBN, DUNE), and neutrino less double beta decay (Next, EXO-200nEXO). A nice review of the history of TPCs and working principles is provided in 105.

Several features of the TPC technology make these detectors a more versatile tool compared to other ionization detectors and explain such a wide popularity. TPCs are the only electronically read detector which deliver simultaneous three-dimensional track information and a measurement of the particle energy loss. Leveraging on both tracking and calorimetry, particle identification (PID) capabilities are enhanced over a wide momentum range.

Historically, the active medium in ionization detectors has been in the gaseous form. Willis and Radeka first proposed the use of liquid-argon ionization chambers as total-absorption detectors in their pioneer work of 1974 [95] Carlo Rubbia imported

this concept to neutrino detection with the ICARUS experiment [118], in 1977. Using nobles elements in the liquid form for neutrino detectors is advantageous for several reasons. The density of liquids is $\sim 1000$ times greater than gases, augmenting the 


\begin{tabular}{|l|c|c|}
\hline Element & LAr & LXe \\
\hline \hline Atomic Number & 18 & 54 \\
Atomic weight A & 40 & 131 \\
Boiling Point Tb at 1 atm & $87.3 \mathrm{~K}$ & $165.0 \mathrm{~K}$ \\
Density & $1.4 \mathrm{~g} / \mathrm{cm}^{3}$ & $3.0 \mathrm{~g} / \mathrm{cm}^{3}$ \\
Radiation length & $14.0 \mathrm{~cm}$ & $2.8 \mathrm{~cm}$ \\
Moliere Radius & $10.0 \mathrm{~cm}$ & $5.7 \mathrm{~cm}$ \\
Work function & $23.6 \mathrm{eV}$ & $15.6 \mathrm{eV}$ \\
Electron Mobility at $E_{\text {field }}=10^{4} \mathrm{~V} / \mathrm{m}$ & $0.047 \mathrm{~m}^{2} / \mathrm{Vs}$ & $0.22 \mathrm{~m} / \mathrm{Vs}$ \\
Average dE/dx MIP & $2.1 \mathrm{MeV} / \mathrm{cm}$ & $3.8 \mathrm{MeV} / \mathrm{cm}$ \\
Average Scintillation Light Yield & $40000 \gamma / \mathrm{MeV}$ & $42000 \gamma / \mathrm{MeV}$ \\
Scintillation $\lambda$ & $128 \mathrm{~nm}$ & $175 \mathrm{~nm}$ \\
\hline
\end{tabular}

Table 2.1: LAr, LXe summary of properties relevant for neutrino detectors.

number of targets for neutrino's interaction in the same volume, in a effort to balance the smallness of neutrino cross section. Since the energy loss of charged particle is proportional to the target material density, as shown in the Bethe-Block equation (eq. 2.1), the increased density reflects into a proportionally higher energy loss, enhancing the calorimetry capability of detectors with a liquid active medium. Additionally, the ionization energy of liquids is smaller than gasses by the order of tens of eV. Thus, at the passage of charged particles, liquids generally produce more ionization electrons than gases for the same deposited energy, forcing the particles to deposit more energy in a shorter range. The downside of using noble liquid elements in experiments is that they require expensive cryogenic systems to cool the gas until it transitions to its liquid form. The properties of liquid argon in comparison liquid xenon - a popular choice for dark matter and neutrinoless double beta decay detectors - are summarized in table 2.1. Albeit xenon would be more desirable than argon given some superior properties such as lower ionization energy and higher density and light yield, argon relative abundance abates the cost of argon compared to xenon, making argon a more viable choice for the construction of ton (and kilo-ton) scale neutrino detectors.

LArTPCs are some times referred as to "electronic" bubble-chambers, for the similarity in the tracking and energy resolution which is coupled with an electronic 
readout of the imaging information in LArTPCs. Compared to these historic detectors however, LArTPC bestow tridimensional tracking, calorimetry and a self triggering mechanism provided by the scintillation light in the liquid argon. An event display of a $\nu_{\mu} \mathrm{CC}$ interaction candidate in the MicroBooNE detector is shown in picture 2.1 to display the level of spatial details these detectors are capable of; the color scale of the image is proportional to the energy deposited, hinting to these calorimetry capabilities of the detectors.

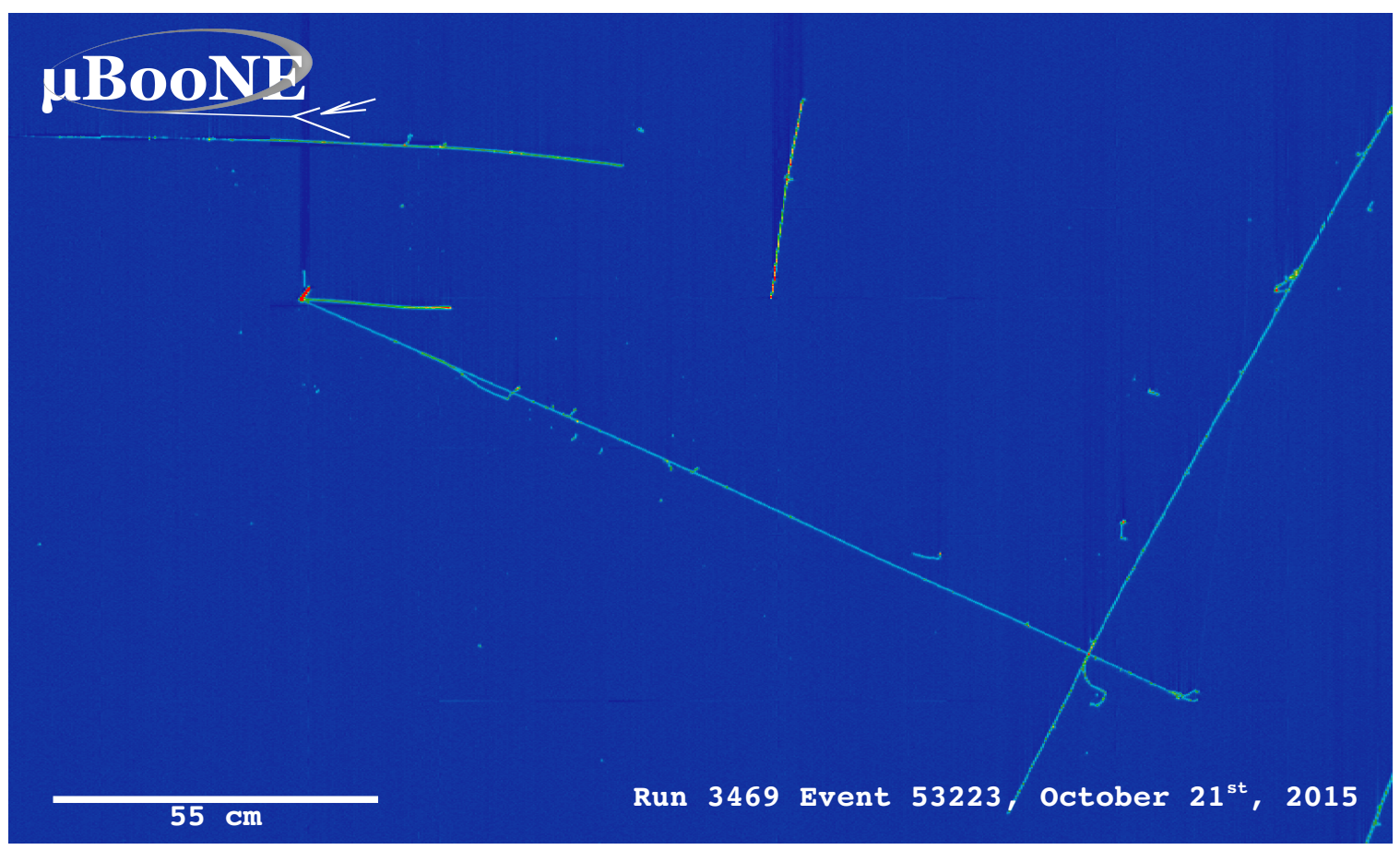

Figure 2.1: Event display of a $\nu_{\mu} \mathrm{CC}$ interaction candidate in the MicroBooNE detector.

\subsubsection{LArTPC: Principles of Operation}

To the bare bones, a LArTPC is a bulk of liquid argon sandwiched in a flat capacitor, equipped with a light collection system, as the cartoon in 2.2 shows. A uniform electric field of the order of $500 \mathrm{~V} / \mathrm{cm}$ is maintained constant between the conductive faces and field shaping rings are used to avoid fringing fields. The anode is sensitive to ionization charge and it is usually made of two or more planes segmented into 


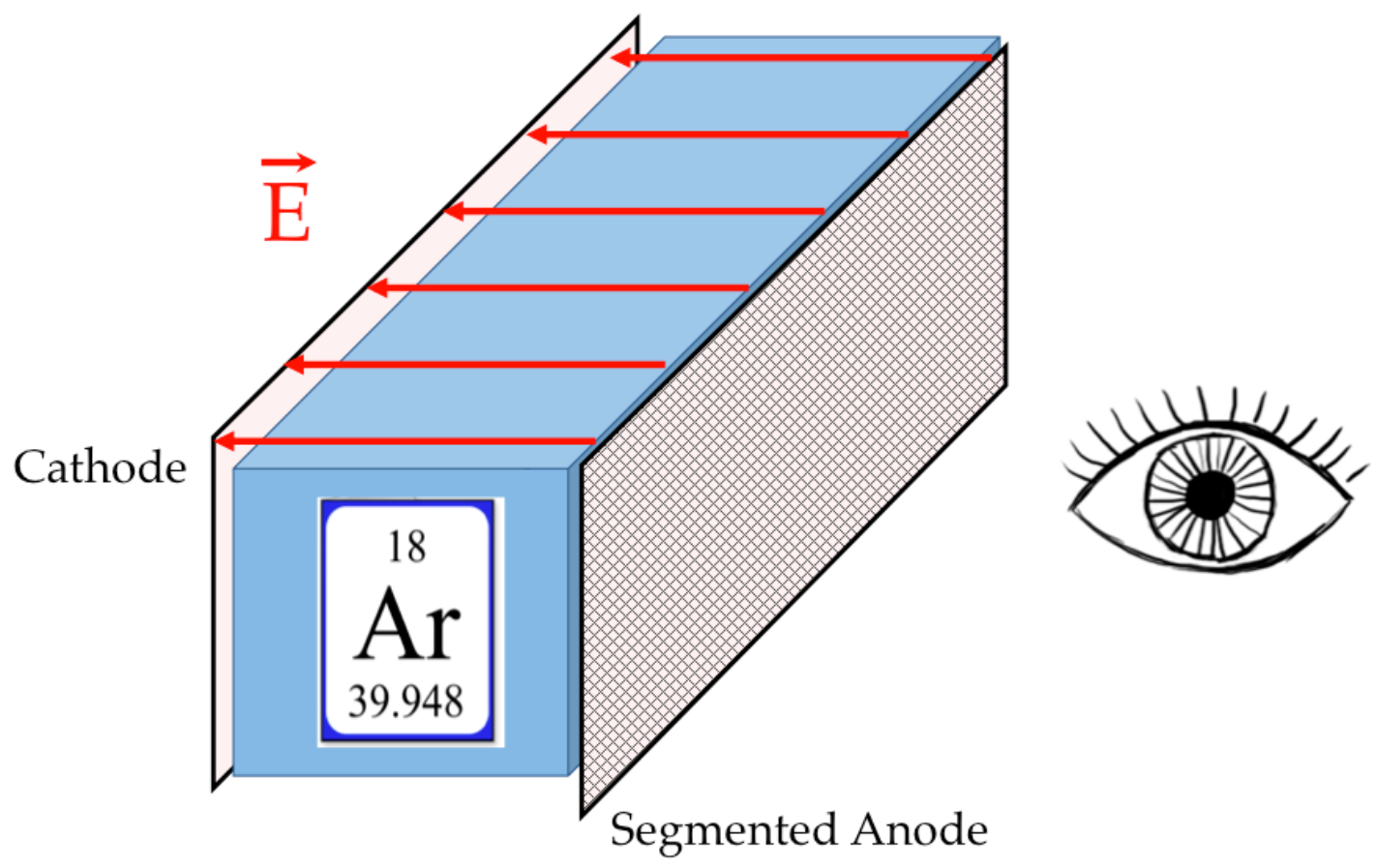

Figure 2.2: A cartoonish sketch of a LArTPC.

several hundreds parallel sense wires a few millimeters apart; different geometries for the anode segmentation are under study [20].

Argon ionization and scintillation are the processes leveraged to detect particles in the LArTPC active volume. When a ionizing radiation traverses the argon active volume it leaves a trail of ionization electrons along its trajectory and it excites the argon producing scintillation light - details on the production and detection of ionization charge and scintillation light are provided in 2.1.4. The optical detector sees the argon scintillation light in matters of nanoseconds. This flash of light determines the start time of an event in the chamber, $t_{0}$. The uniform electric field drifts the ionization electrons from the production point towards the anode in order of hundreds of microseconds or more depending on the chamber dimensions ${ }^{1}$, The anode sense

1. The ionized argon also drifts, but in the opposite directions compared to the electrons. Since the drift time is proportional to the particle mass, the ions' drift time is much longer than the electrons'. Ionized argon is collected on the cathode which is not instrumented, so it is not used to infer information about the interactions in the chamber. 
wires see either an induced current by the drifting ionization charge (on induction planes) or an injection of such charge (collection plane). An appropriate choice of the voltage bias on each wire plane assures ideal charge transparency, so that all the ionization charge is collected on the collection plane and none on the induction planes.

The arrival time of the charge on the anode sense wires is used to measure the position of the original ionizing radiation in the drift direction. In fact, since the constant electric field implies that the drift velocity is also constant, the position of the original ionization is simply given by the multiplication of the drift velocity by the drift time, where the "drift time" is the difference between $t_{0}$ and the charge arrival time on the wire planes. The spatial resolution on this dimension is limited by the time resolution of the electronics or by longitudinal diffusion of the electrons. The spatial information on the different wire planes maps a bi-dimensional projection of the interaction pattern in the plane perpendicular to the drift direction. The spacial resolution on this dimension is limited by the transverse electron diffusion in argon and by the grain of the anode segmentation, i.e. the spacing between the wires in the sense planes 86]. The off-line combination of the 2-D information on the wire planes with the timing information allows for the 3D reconstruction of the event in the chamber.

Since the charge deposited by the ionizing radiation is proportional to the deposited energy and the charge collected on the sense plane is a function of the deposited charge, LArTPCs allow the measurement of the energy deposit in the active volume. Effects due to the presence of free charge and impurities in the active volume, such as a finite electron lifetime, recombination and space charge, complicate the relationship between deposited and collected charge affecting the measurement of the particle's energy, as described in the next section. 


\subsubsection{Liquid Argon: Ionization Charge}

The mean rate of energy loss by moderately relativistic elementary charge particles heavier than electrons is well described by the modified Bethe-Bloch [36] equation

$$
-\frac{d E}{d x}=K z^{2} \frac{Z}{A} \varrho \frac{1}{\beta^{2}}\left[\frac{1}{2} \ln \frac{2 m_{e} c^{2} \beta^{2} \gamma^{2} T_{\max }}{I^{2}}-\beta^{2}-\frac{\delta}{2}\right]
$$

where $z$ is the number of unit charge of the ionizing radiation, $Z, A$ and $\varrho$ are the atomic number, mass number and density of the medium, $m_{e}$ is the electron mass, $\gamma=\frac{\beta}{\sqrt{1-\beta^{2}}}$ is the Lorentz factor of the ionizing radiation, $T_{\max }$ is the maximum kinetic energy which can be imparted to a free electron in a single collision, $I$ is the mean excitation energy on $\mathrm{eV}, \delta$ is the density correction and $K=0.307075 \mathrm{MeV} \mathrm{g}^{-1} \mathrm{~cm}^{2}$ is a numerical conversion factor. The Bethe-Bloch treats the energy loss by an ionizing radiation via quantum-mechanical collisions producing ionization or an excitation in the medium as an uniform and continuous process. The density correction terms becomes relevant for incident particle with high energy, where screening effects due to the polarization of the medium by high energy particles occur.

Excitation and ionization of the detector medium occur in similar amounts. Since the ionizing collisions occur randomly, we can parametrize their number $k$ in a segment of length $s$ along the track with a Poissonian function

$$
P(k)=\frac{s^{k}}{k ! \lambda^{k}} e^{-s / \lambda},
$$

where $\lambda=1 / N_{e} \sigma_{i}$, with $N_{e}$ being the electron density of $\sigma_{i}$ the ionization crosssection per electron. About $66 \%$ of the ionizing collisions in argon produce only a single electron/ion pair 105]; in the other cases, the transferred kinetic energy is enough for the primary electron to liberate one or more secondary electrons, which usually stay close to the original pair. Occasionally, electrons can receive enough 
energy to be ejected with high energy, forming a so-called " $\delta$-ray" : a detectable short track off the particle trajectory, as shown in figure 2.3 . The average number of $\delta$-ray with energy $\mathrm{E}>\mathrm{E}_{0}$ per $\mathrm{cm}$ follows the empirical form

$$
P\left(E>E_{0}\right) \sim \frac{y}{\beta^{2} E_{0}}
$$

where $y$ is an empirical factor depending on the medium (0.114 for gaseous Ar), and $\beta$ is $v / c$.

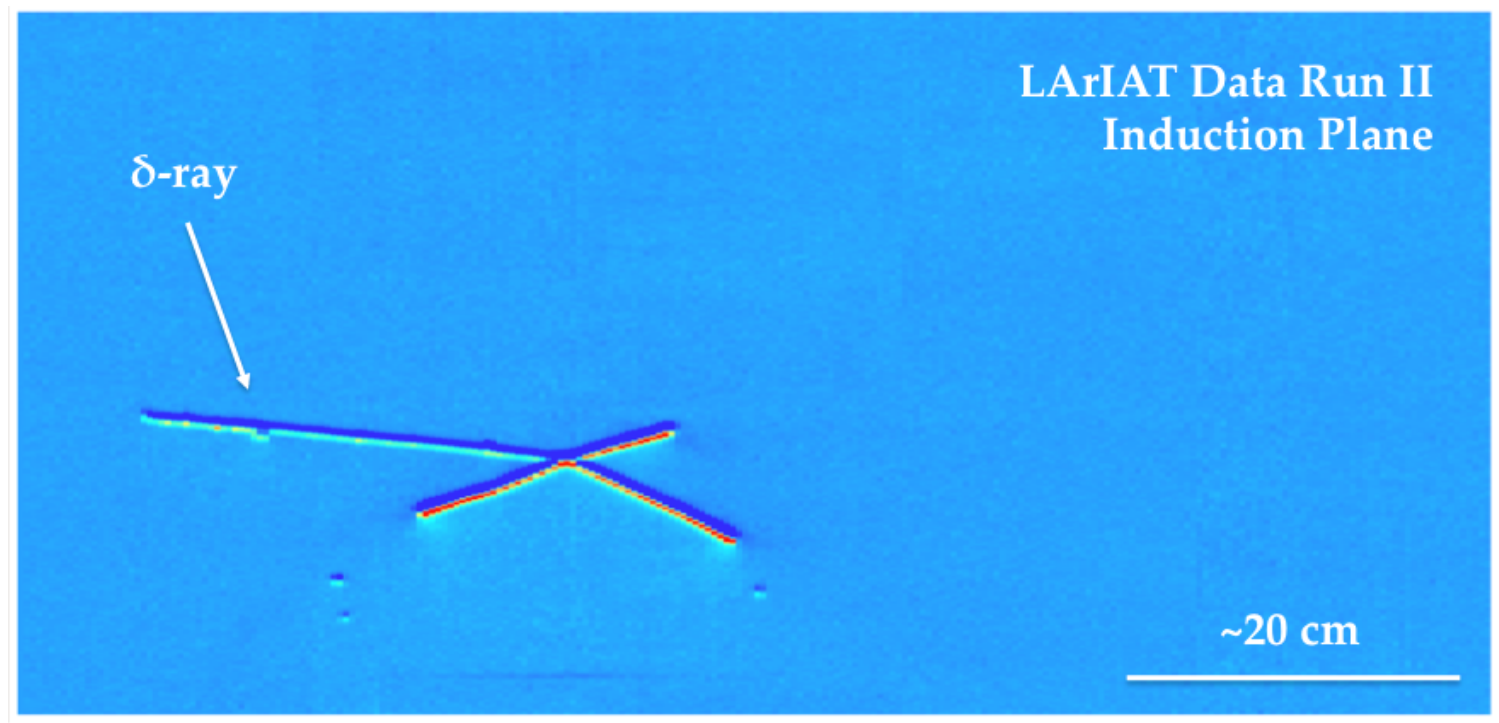

Figure 2.3: Event display for a LArIAT pion absorption candidate on the induction plane, with highlighted delta ray.

\section{Purity \& Electron Life Time}

The presence of electronegative contaminants in liquid argon, such as oxygen $\mathrm{O}_{2}$ and water $\mathrm{H}_{2} \mathrm{O}$, is particularly pernicious, since these molecules quench the charge produced by the ionizing radiation. Thus, amount of charge per unit of length $d Q / d x$ collected on the collection plane depends on the charge's production point in the detector: ionization produced close to the cathode will see more impurities along its journey to the collection plane than ionization produced close to the anode, resulting 
in greater attenuation of its charge. As a result, the amount of charge collected on the sense wires as a function of the traveled distance follows an exponential decay trend. The traveled distance is generally measured in terms of drift time and the characteristic time constant of the exponential decay is called electron lifetime $\tau_{e}$. Figure 2.4 shows the typical life time for LArIAT data. The procedure to measure the electron lifetime in LArIAT is outlined in [73]. LArIAT small drift distance (47 $\mathrm{cm}$ ) allows for a relatively short electron life time. The life time for bigger detectors such as MicroBooNE, whose drift distance is $2.6 \mathrm{~m}$, needs to be of the order of tens of milliseconds to allow a charge collection usable for physics analyses. Energy reconstruction in LArTPC applies a correction for the finite lifetime to calibrate the detector calorimetric response; details for LArIAT are provided in Section C.

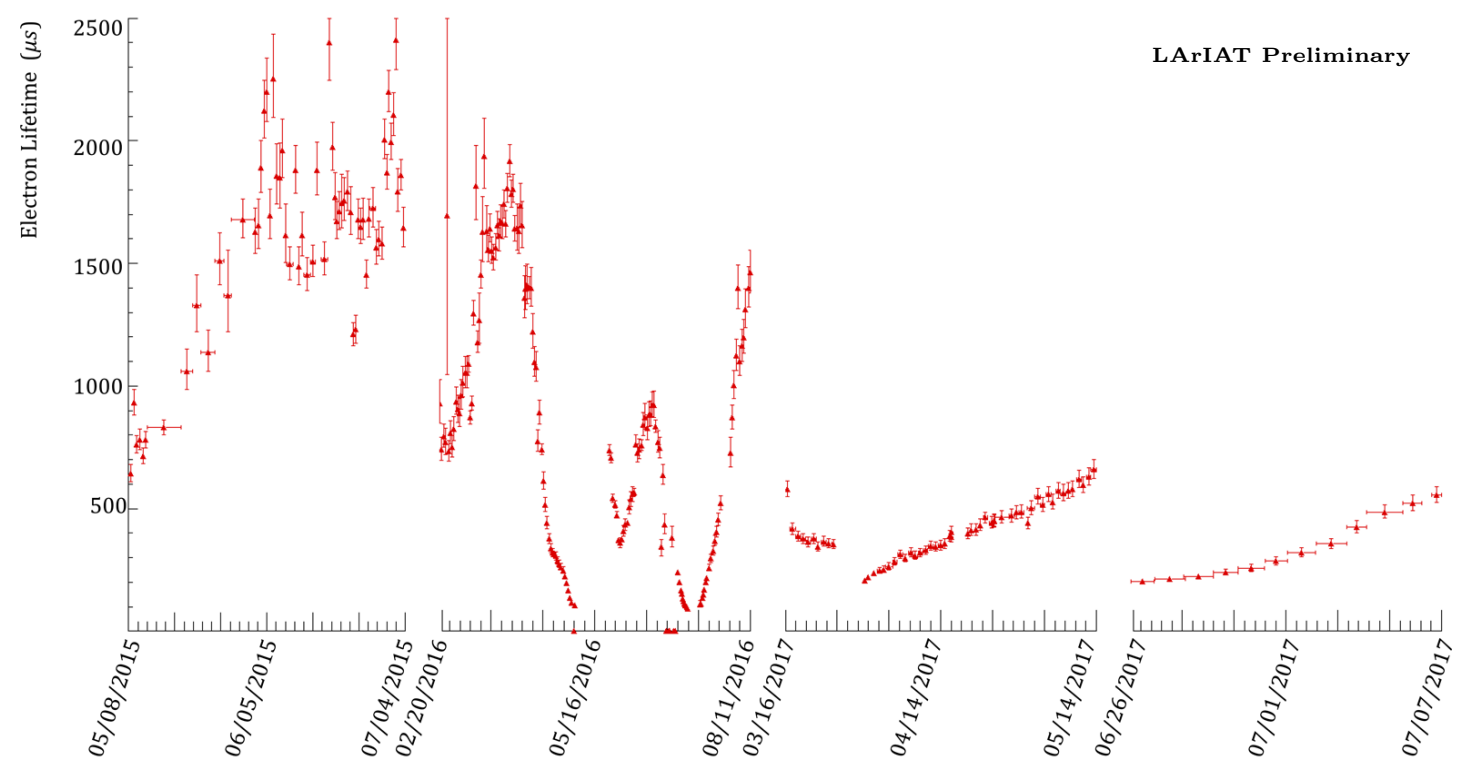

Figure 2.4: Electron lifetime during the LArIAT run period [1]. 
LArTPCs use hermetically sealed and leak-checked vessels to abate the leakage and diffusion of contaminants into the system. The liquid argon filling of the volume occurs after the vessel is evacuated or purged with gaseous argon [62] to reduce remaining gases in the volume. Even so, the construction of a pure tank of argon is unviable, as several sources of impurity remain. In particular, impurities can come from the raw argon supply, the argon filtration system and from the outgassing from internal surfaces. Outgassing is a continuous diffusive process producing contaminants, especially water, even after the vessel is sealed, particularly from materials in the ullage region ${ }^{2}$, Since research-grade argon comes from the industrial distillation of air, the impurities with the highest concentration are nitrogen, oxygen and water, generally maintained under the 1 part per million level by the vendor. Even so, a higher level of purity is necessary to achieve a free electron life time usable in meter scale detectors. Thus, argon is constantly filtered in the cryogenic system, which reduce the oxygen and water contamination to less than 100 parts per trillion. The filtration system depends on the size and drift distance of the experiment and, for experiments on several meters scale, it includes an argon recirculation system [75].

\section{Recombination Effect}

After production, ionization electrons thermalize with the surrounding medium and may recombine with nearby ions. Recombination might occur either between the electron and the parent ion through Coulomb attraction, as described in the geminate theory [115], or thanks to the collective charge density of electrons and ions from multiple ionizations in a cylindrical volume surrounding the particle trajectory, as described in the columnar model [106]. Consideration on the average electron-ion distance and the average ion-ion distance for argon show that the probability of

2. While the liquid argon low temperature reduces outgassing in the liquid, this process remains significant for absorptive material (such as plastic) above the surface of the liquid phase. 
geminate recombination is low; thus recombination in argon is mainly due to collective effects [72. Since protons, kaons and stopping particles present a higher ionization compared to MIPs, recombination effects are more prominent when considering the reconstruction of energy deposited by these particles.

Theoretical descriptions of recombination based on the Birks model and the Box model are provided in [4] and [57], respectively. The Birks model assumes a gaussian spatial distribution around the particle trajectory during the entire recombination phase and identical charge mobility for ions and electrons. The Box model also assumes that electron diffusion and ion mobility are negligible in liquid argon during recombination. In these models, the fraction of ionization electrons surviving recombination is a function of the number of ion-electron pairs per unit length, the electric field, the average ion-electron separation distance after thermalization and the angle of the particle with respect to the direction of the electric field - plus the diffusion coefficient in the Birks model. Given the stringent assumptions, it is perhaps not surprising that these models are in accordance to data only in specific regimes: the Birks model is generally used to describe recombination for low $\mathrm{dE} / \mathrm{dx}$, the Box model for high $\mathrm{dE} / \mathrm{dX}$. In LArTPC, the ICARUS and ArgoNeut experiments have measured recombination in [80] and [72] respectively. Since LArIAT uses the refurbished ArgoNeut TPC and cryostat at the same electric field, LArIAT currently corrects for recombination using the ArgoNeut measured recombination parameters in [72].

\section{Space Charge Effect}

Slow-moving positive argon ions created during ionization can build-up in LArTPC, causing the distortion of the electric field within the detector. This effect, called "space charge effect" leads to a displacement in the reconstructed position of the signal ionization electrons. In surface LArTPCs the space charge effect is primarily due to the rate of ionization produced by cosmic rays which is slowly drifting in the 
chamber at all times. Surface LArTPC of the size of several meters are expected to be modestly impacted from the space charge effect, where charge build-up create anisotropy of the electric field magnitude of the order of $5 \%$ at a drift field of 500 $\mathrm{V} / \mathrm{cm}$ [11]. The smallness of the LArIAT drift volume and its relatively high electric field are such that the effect of space charge is expected to be negligible.

\subsubsection{Liquid Argon: Scintillation Light}

Liquid argon emits scintillation light at the passage of charged particles. LArTPCs leverage this property to determine when the ionization charge begins to drift towards the anode plane.

\section{Scintillation Process}

Scintillation light in argon peaks in the ultraviolet at a $128 \mathrm{~nm}$, shown in comparison to Xenon and Kypton in Figure 2.5, from [45]. The light yield collected by the optical detector depends on the argon purity, the electric field, the $\mathrm{dE} / \mathrm{dx}$ and particle type, averaging at the tens of thousands of photons per $\mathrm{MeV}$.

The de-excitation of Rydberg dimers in the argon is responsible for the scintillation light. Rydberg dimers exist in two states: singlets and a triplets. The time constant for the singlet radiative decay is $6 \mathrm{~ns}$, resulting in a prompt component for the scintillation light. The decay of the triplet is delayed by intersystem crossing, producing a slow component with a time constant of $\sim 1500$ ns. "Self-trapped exciton luminescence" and "recombination luminescence" are the two processes responsible for the creation of the Rydberg dimers [108]. In the first process, a charged particle excites an argon atom which becomes self-trapped in the surrounding bulk of argon, forming a dimer; the dimer is in the singlet state $65 \%$ of the times and in the triplet state $35 \%$ of the times. In case of recombination luminescence, the charged particle transfers enough energy to ionize the argon. The argon ion forms a charged argon dimer state, 


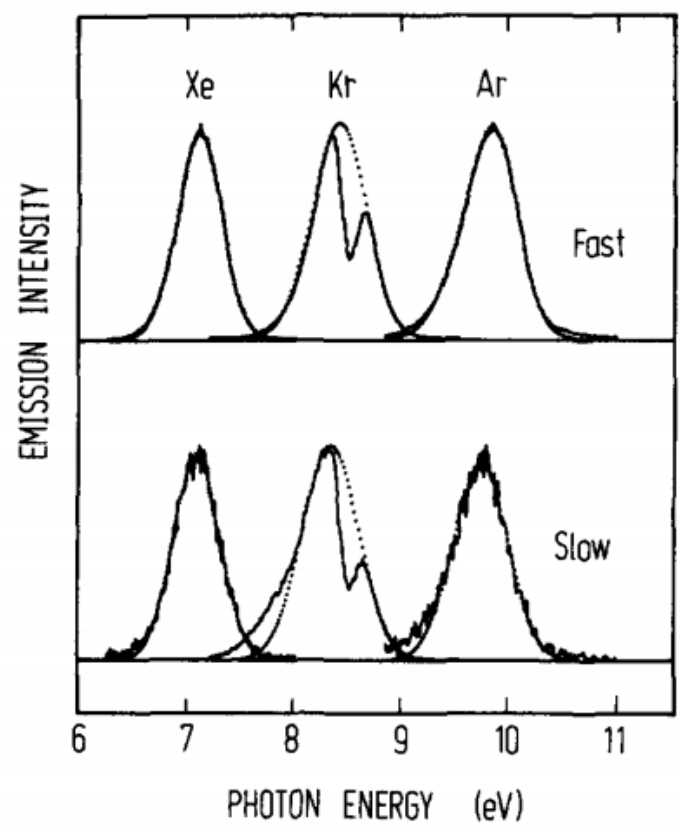

Figure 2.5: Emission spectra of the fast and slow emission components in Xenon, Kypton and Argon according to [45]. The dotted lines correspond to the Gaussian fits.

which quickly recombines with the thermalized free electron cloud. Excimer states are produced in the recombination, roughly half in the singlet and half in the triplet state. The light yield dependency on the electric field, on the $\mathrm{dE} / \mathrm{dx}$ and particle type derives from the role of free charge in the recombination luminescence process. The spacial separation between the argon ions and the free electron cloud depends on the electric field. On one hand, a strong electric field diminishes the recombination probability, leading to a smaller light yield; on the other, it increases the free charge drifting towards the anode plane. Hence, the amount of measurable charge and light anti-correlates as a function of the electric field. Ionizing particles in the argon modify the local density of both free electrons and ions depending on their $\mathrm{dE} / \mathrm{dx}$. Since the recombination rate is proportional to the square of the local ionization density, highly ionizing particles boost recombination and the subsequent light yield compared to MIPs. The possibility to leverage this dependency for pulseshape-based particle identification has been shown in 65, 93. 


\section{Effects Modifying the Light Yield}

The production mechanism through emission from bound excimer states implies that argon is transparent to its own scintillation light. In fact, the photons emitted from these metastable states are not energetic enough to re-excite the argon bulk, greatly suppressing absorption mechanisms. In a LArTPC however, several processes modify the light yield in between the location where light is produced and the optical detector. In a hypothetical pure tank of argon, Rayleigh scattering would be the most important processes modifying the light yield. Rayleigh scattering changes the path of light propagation in argon, prolonging the time between light production and detection. The scattering length has been measured to be $66 \mathrm{~cm} \mathrm{[66],} \mathrm{shorter} \mathrm{than} \mathrm{the} \mathrm{theoretical}$ prediction of $\sim 90 \mathrm{~cm}[79]$; this value is short enough to be relevant for the current size of LArTPCs detectors. In fact, Rayleigh scattering worsens the resolution on $t_{0}$, the start time for charge drifting, and alters the light directionality, complicating the matching between light and charge coming from the same object in case of multiple charged particles in the detector.

Traces of impurities in argon such as oxygen, water and nitrogen also affect the light yield, mainly via absorption and quenching mechanisms. Absorption occurs as the interaction of a $128 \mathrm{~nm}$ photon directly with the impurity dissolved in the liquid argon. Differently, quenching occurs as the interaction of an argon excimer and an impurity, where the excimer transfers its excitation to the impurity and dissociates non-radiatively. Given this mechanism, it is evident how quenching is both a function of the impurity concentrations and the excimer lifetime. Since the triplet states live much longer than the singlet states, quenching occurs mainly on triplet states, affecting primarily the slow component of the light, reducing the scintillation yield and a shortening of the scintillation time constants.

The stringent constraints for the electron life time limit the presence of oxygen and water to such a low level that both absorption and quenching on these impurity is not 
expected to be significant. Contrarily, the nitrogen level is not bound by the electron life time constraints - nitrogen being an inert gas, expensive to filter. Thus, nitrogen is often present at the level provided by the vendor. The effects of nitrogen on argon scintillation light have been studied in the WArP R\&D program and at several test stands. The quenching process induced by nitrogen in liquid Ar has been measured to be proportional to the nitrogen concentration, with a rate constant of $\sim 0.11$ $\mu s^{-1} \mathrm{ppm}^{-1}$; appreciable decreasing in lifetime and relative amplitude of the slow component have been shown for contamination as high as a few ppm of nitrogen [70]. For a nitrogen concentration of 2 parts per million, typical of the current generation of LArTPC, the attenuation length due to nitrogen has been measured to be $\sim 30$ meters [26].

\section{Wavelength Shifting of LAr Scintillation Light}

Liquid argon scintillation light is invisible for most optical detectors deployed in a LArTPC, such as cryogenic PMTs and SiPMs, since a wavelength of $128 \mathrm{~nm}$ is generally too short to be absorbed from most in glasses, polymers and semiconductor materials. Research on prototype SiPMs absorbing directly VUV light and their deployment in noble gasses experiment is ongoing but not mature 67]. Thus, experiments need to shift the wavelength of scintillation light to be able to detect it. Albeit deployed in different ways, neutrinos and dark matter experiments commonly use 1,1,4,4-tetraphenyl-butadiene (TPB) to shift the scintillation light. TPB absorbs the vacuum ultraviolet (VUV) light and emits in the visible at $\sim 425 \mathrm{~nm}$ 94, with a ratio of visible photon emitted per VUV photon absorbed of $\sim 1.2: 1$ [92].

Neutrino experiments typically coat their optical detector system evaporating a layer of TPB either directly on the PMTs glass surface or on acrylic plates mounted in front of the PMTs [76]; this technique allows the fast detection light coming directly from the neutrino interaction. Dark matter experiments typically evaporate TPB on 
reflective foils mounted on the inside walls of the sensitive volume and detect the light after it has been reflected; this technique leads to a higher and more uniform light yield, though scattering effects for both the visible and VUV light augment the propagation time and hinder directionality information [33]. In order to take advantage of both these techniques, hybrid systems with PMT coating and foils are being considered for the next generation of large neutrino detectors.

\subsubsection{Signal Processing and Event Reconstruction}

In this section we illustrate the processing and reconstruction chain of the TPC signals, from the pulses on the sense wire to the construction of three dimensional objects with associated calorimetry. Different experiments can chose different software packages for their off line signal processing and event reconstruction, but a popular choice for US based LArTPCs is LArSoft [8]. Based on the Art framework [34], LArSoft is an event-based toolkit to perform simulation, analysis and reconstruction of LArTPCs events.

LArTPC signal processing develops in several consecutive stages that we summarize here in the following categories: Deconvolution, Hit Reconstruction, 2D Clustering, 3D Tracking, Calorimetry Reconstruction. A visualization of the signal processing workflow is shown in figure 2.6 .

Deconvolution. Induction and collection planes have different field responses, given the different nature of the signals on these planes: the wires on the induction planes see the inductive signal of the drifting charge, while the wires on the collection

planes see the current derived from the charge entering the conductor. Thus, signals on the induction plane are bi-polar pulse and signal on the collection plane are unipolar pulses, see Figure 2.6 panel a). The first step in signal processing is deconvolution, 

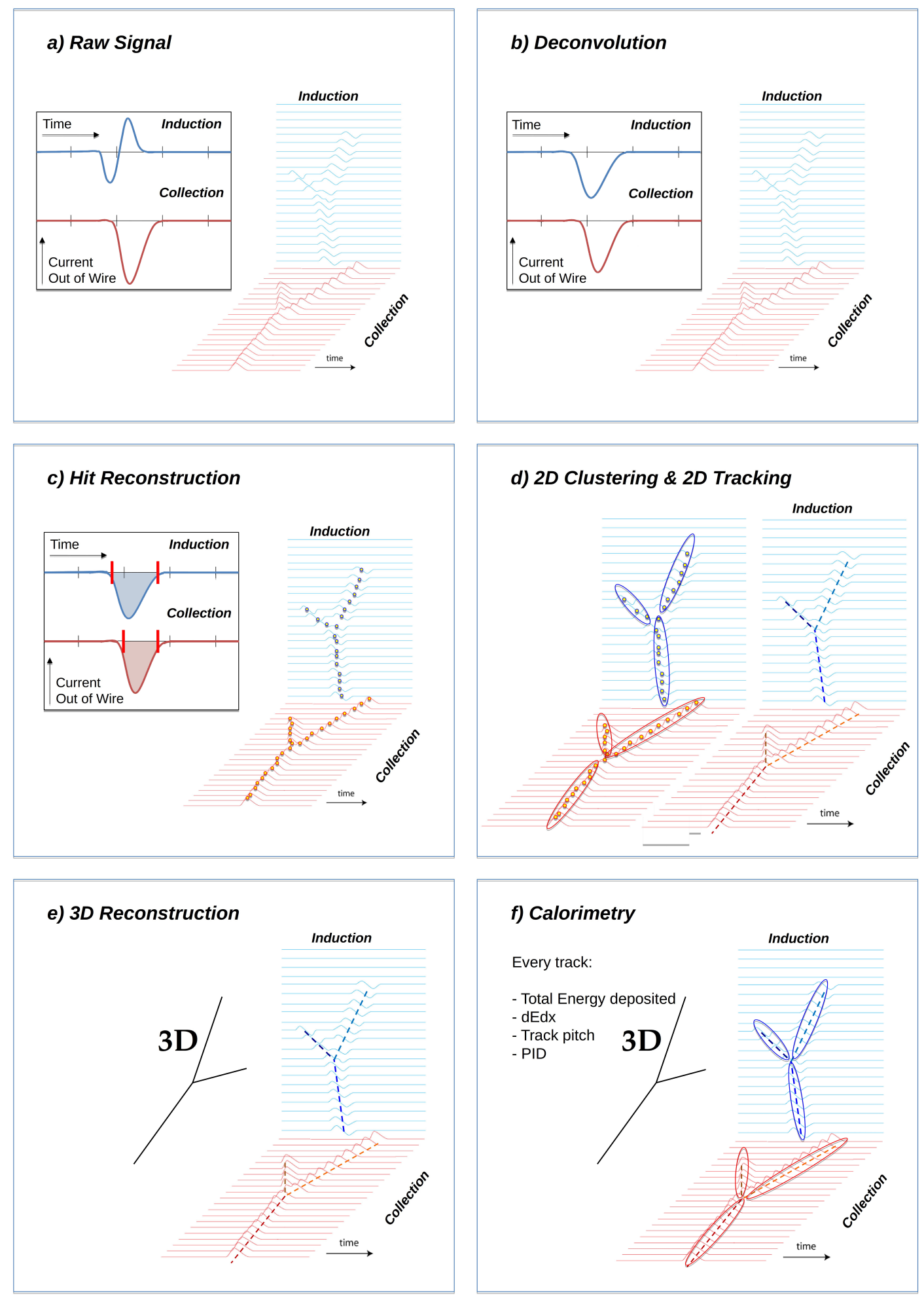

Figure 2.6: A scheme of a typical signal processing workflow in LArSoft. 
that is a series of off-line algorithms geared towards undoing the detector effects. The result of the deconvolution step is the production of a comparable set waveforms on all planes presenting unipolar, approximately gaussian-like pulses (Figure 2.6 panel b). Signal from all planes are treated on equal footage beyond this point. Some LArTPC apply noise filtering in the frequency domain just after the deconvolution to clean up wire cross talk. Since signals from the LArIAT TPC are extremely clean, noise filtering is not necessary.

Hit Reconstruction. The second stage of the signal processing is the reconstruction of hits, indicating an energy deposition in the detector. A peak finder scans the deconvolved TPC waveforms for each wire on the whole readout time looking for spikes above the waveform's baseline. It then fits these peaks with gaussian shapes and stores the fit parameters such as the quality of the fit, the peak time, height and area under the gaussian fit. The information resulting from this process on a single spike form a single reconstructed "hit", see Figure 2.6 panel c). The next steps in the event reconstruction chain will then decide which hits to use according to their goodness of fit. It is important to notice how the height and width of the hit depend on the topology of the event: for example, a particle running parallel to the wire planes will leave a series of narrow individual hits, one on each consecutive wire, while a particle traveling towards the planes will leave long, wide hits on very few wires. The height of the hits and their integral is proportional to the charge collected on the wire, so it depends on the particle type.

The event reconstruction chain uses collection of hits to form more complex objects associated with the particles in the detector. The development of different approaches to accomplish this task is an extremely hot topic in LArTPC event reconstruction which spans from more traditional approaches such as line-clustering [3] to the use of 
machine learning tools $[74]$. Generally speaking, the scope of hit clustering and event reconstruction is to provide shower-like or track like-objects with an associated energy reconstruction. This is because different particles have different topology in the detector - electrons and photon create electromagnetic showers, resulting in shower-like topologies, while muons and hadrons leave track-like signals. For the scope of this thesis, we will describe only LArIAT's approach to track reconstruction even if we recognize the breath of LArTPC event reconstruction is much wider. We are interested in the reconstruction of pions and kaons in the active volume, whose topology is track-like.

2D Clustering Reconstruction. The LArIAT reconstruction of track-like objects starts by clustering hits on the collection and induction planes separately with the use of the TrajCluster clustering package [2]. TrajCluster looks for a collection of hits in the wire-time 2D space which can be described with a line-like 2D trajectory. TrajCluster reconstructs trajectories by adding trajectory points to the leading edge of the trajectory while stepping through the 2D space of hits. Several factors determine whether a hit is added to the trajectory, including but not limited to

1. the goodness of the fit of the single hit,

2. the charge of the hit compared to the average charge and RMS of the hits already forming the trajectory,

3. the goodness of trajectory fit with and without the hit addition,

4. the angle between the two lines formed by the collection of hits before and after the considered hit in the trajectory.

The final product of this reconstruction stage is the collection of bidimensional clusters on each wire plane, see Figure 2.6 panel d). 
3D Tracking. The 3D tracking set of algorithms uses clusters close in time on the induction and collection planes as starting point to form a 3D track. Firstly, it constructs a tentative 3D trajectory using the edges of the clusters. Then, it projects back the tentative trajectory on to the planes and adjusts the parameters of the 3D track fit such that they minimize the distance between the fit projections and the track hits in all wire planes simultaneously. Tridimensional tracking can use multiple clusters in one plane, but it can never break them into smaller groups of hits. This algorithm was first developed for the ICARUS collaboration [63]. The final product of this reconstruction stage is the formation of tridimensional objects in the TPC active volume, see Figure 2.6 panel e).

Calorimetry. The last step in the event reconstruction chain is to assign calorimetric information to the track (or shower) objects. Calorimetry is performed separately on the different planes. A multi-step procedure is needed to retrieve the energy deposited in the TPC from the charge seen by the wires. For each hit associated with the track object, the calorimetry algorithms calculate the charge seen on every wire using the area underneath the gaussian fit; then, they correct this raw charge by the electron life time, the electronic noise on the considered wire and the recombination effect. Lastly an overall calibration of the energy, explained in detail in section C, is applied and the calorimetric information for the given track is assigned. Even if calorimetry is done in 2D, it benefits from the 3D tracking information; typical objects available after the calorimetric reconstruction are the total energy deposited by the particle and its stopping power $d E / d x$ at each "track pitch", i.e. at each segment between two 3D points projected on the wire plane. 


\subsection{The Intensity Frontier Program}

This section highlights the role of Liquid Argon Time Projection Chambers at the Intensity frontier. In particular, we show the prospects for the exploration of neutrino physics (Section 2.2.1) and GUT models (Section 2.2.2) in current and forthcoming LAr experiments. In Section 2.2.3, we introduce LArIAT and its role in the Intensity Frontier panorama.

\subsubsection{Prospects for LArTPCs in Neutrino Physics: SBN and DUNE}

The ArgoNeut experiment 29] together with the LAr R\&D experiments TallBo and the Yale TPC initiated the US LArTPC neutrino program. Following the success of the ArgoNeut small TPC on the NuMI beam, a wide program of LArTPCs on neutrino beams has flourished. The construction of LArTPCs as near and far detectors at different baseline allows for the exploration of some of the fundamental questions in neutrino physics today illustrated in section 1.3.1.

The Short-Baseline Neutrino (SBN) [64] program at Fermilab is tasked with con-

clusively addressing the nature of the "LSND and MiniBooNE anomalies" 15, 16, 32, resolving the mystery of sterile neutrinos at the $\mathrm{eV}^{2}$ scale. The SBN program is comprised of three surface LArTPCs positioned on the Booster Neutrino Beam at different distances from the neutrino production in order to fully exploit the $\mathrm{L} / \mathrm{E}$ dependence of the oscillation pattern: SBND (110 m from the decay pipe), MicroBooNE $(470 \mathrm{~m})$, and ICARUS $(600 \mathrm{~m})$. Within the oscillation context, the choice of the LArTPC technology for the SBN detectors changes the set of systematics with respect to LSND and MiniBooNE, whose detection techniques were both based on Cherenkov light. In particular, LArTPCs provide excellent electron/photon separation [77] lacking in Cherenkov detectors which can be leveraged to reduce the photon 
background from neutral current interactions in $\nu_{e}$ searches. MicroBooNE [75], the first detector of the SBN program to be fully operational, started its first neutrino run in October 2015. MicroBooNE is a 85 ton active volume LArTPC, single drift chamber with TPC dimensions of $2.6 \mathrm{~m}$ (drift) x $2.3 \mathrm{~m}$ (heigh) x $10.4 \mathrm{~m}$ (depth). MicroBooNE is positioned at a the same energy and very similar baseline on the Booster neutrino beam as MiniBooNE; MicroBooNE has the scope to directly cross check the MiniBooNE oscillation measurement. In case MicroBooNE confirms the presence of the "low energy excess" (LEE) anomaly, SBND and ICARUS will confirm the LEE is due to oscillations (length dependent). In case MicroBooNE does not confirm the LEE, the SBN program will fully address the entire allowed parameter space. SBND and ICARUS are both dual drift chambers, whose active volume is respectively 112 ton and 600 ton. ICARUS is scheduled to become operational by the end of 2018 and SBND shortly after. Besides the oscillation analysis, the second main goals of SBN is to perform an extensive campaign of neutrino cross section measurements in argon. Given the importance of nuclear effects in (relatively) heavy materials, as discussed in section 1.2.3, both the oscillation analysis of the SBN program and the measurements of neutrino properties in DUNE will benefit from such a campaign.

On a different neutrino beam and baseline, the DUNE experiment, née LBNE 28], is the flagship experiment on the medium-long term of US-based neutrino physics, scheduled to start data taking in 2026. Shooting neutrinos from Fermilab for 800 miles to the SURF laboratory in South Dakota, DUNE is tasked with preforming conclusive measurements of $\mathrm{CP}$ violation in the lepton sector, the neutrino mass ordering and the $\theta_{23}$ octant. The DUNE far detector is comprised of four $10 \mathrm{kton}$ fiducial volume LArTPCs, roughly of dimensions of $19 \mathrm{~m}$ (horizontally) x $18 \mathrm{~m}$ (vertically) x $66 \mathrm{~m}$ (depth). 


\subsubsection{Prospects for LArTPCs in GUT Physics: DUNE}

The experimental exploration of a manifestation of Grand Unified Theory is possible in DUNE thanks to its sheer mass. In particular, proton decay searches are a capital topic of DUNE's wide non-accelerator physics program. The key elements for a rare decay experiment are: massive active volume, long exposure, high identification efficiency and low background. Figure 2.7 shows the current best experimental limits on nucleon decay lifetime over branching ratio (dots). Historically, the dominant technology used in these searches has been water Cherenkov detectors: all the best experimental limits on every decay mode are indeed set by Super-Kamiokande [58, 91. As shown in section 1.3.2, different family of GUTs predict the proton to decay in different modes. In particular, SUSY flavored GUTs prefer the presence of kaons in the decay products, e.g. $p \rightarrow K^{+} \bar{\nu}$. It is particularly important to notice that

the kaon energy for the proton decay mode $p \rightarrow K^{+} \bar{\nu}$ is under Cherenkov threshold in water. Thus, Super-Kamiokande set the limit on the lifetime for the $p \rightarrow K^{+} \bar{\nu}$ mode by relying on photons from nuclear de-excitation and on the muon tagging in the kaon decay leptonic mode. For this reason, an attractive alternative approach to identifying nucleon decay is the use of a LArTPCs, where the kaon is directly visible in the detector. According to [28], DUNE will have an active volume large enough, have sufficient shielding from the surface, and will run for lengths of time sufficient to compete with Hyper-K, opening up the opportunity for the discovery of nucleon decay.

\subsubsection{Enabling the next generation of discoveries: LArIAT}

LArIAT, a small LArTPC in a test beam, is designed to perform an extensive physics campaign centered on charged particle cross section measurements while characterizing the detector performance for future LArTPCs. Since LArTPCs represent the most advanced experiments for physics at the Intensity Frontier, their complex technology 


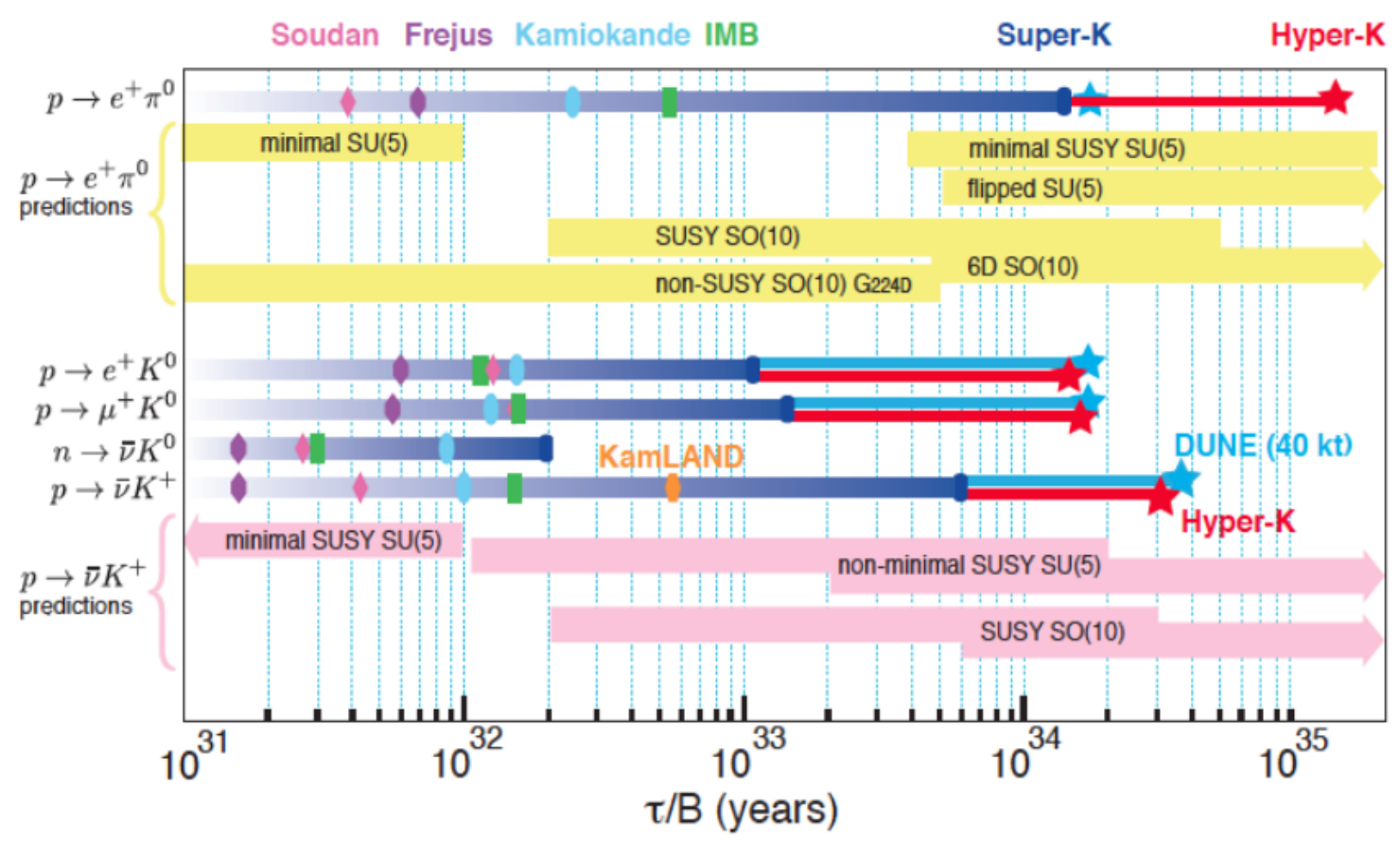

Figure 2.7: Proton decay lifetime limits from passed and future experiments.

needs a thorough calibration and dedicated measurements of some key quantities to achieve the precision required for the next generation of discoveries. LArIAT's goal is to provide such calibration and dedicated measurements. The LArIAT LArTPC is deployed in a dedicated calibration test beamline at Fermilab. We use the LArIAT beamline to characterize the charge particles before they enter the TPC: the particle type and initial momentum is known from beamline information. The precise calorimetric energy reconstruction of the LArTPC technology enables the measurement of the total differential cross section for tagged hadrons. The Pion-Nucleus and KaonNucleus total hadronic interaction cross section have never been measured before in argon and they are a fundamental step to shed light on light meson interaction in nuclei per se, while providing a key input to neutrino physics and proton decay studies in future LArTPC experiments like SBN and DUNE.

In order to showcase LArIAT's utility to SBN and DUNE, we illustrate briefly two comparisons as examples: one regarding neutrino interactions and the second 
regarding proton decay studies.

The left side of figure 2.8 shows the distribution of products in momentum spectrum and particle type as simulated in a $\nu_{e} \mathrm{CC}$ interaction in DUNE (according to [14]); the range of these distribution is to compare with the momentum distribution of light particles in the LArIAT beamline - shown on the right side of figure 2.8. The momentum spectrum in the LArIAT beamline for electrons, muons and pions - the most abundant particles produced in a $\nu_{e} \mathrm{CC}$ interaction - covers a wide range of the expected momentum distribution in a neutrino event.

The signature of a proton decay event in the "LAr golden mode" is the presence of a single kaon of about $400 \mathrm{MeV}$ in the detector; the momentum spectrum of the kaon pre and post FSI in such an event as simulated by GENIE is shown on the left side of figure 2.9, The right side of figure 2.9 shows the momentum spectrum of kaons in the LArIAT beamline. Kaons arriving to the LArIAT TPC are ideal for proton decay studies, since their momentum in the beamline is just above the typical momentum for kaons in a proton decay event: the majority of LArIAT kaons slow down in the TPC enough to enter the desired momentum window.
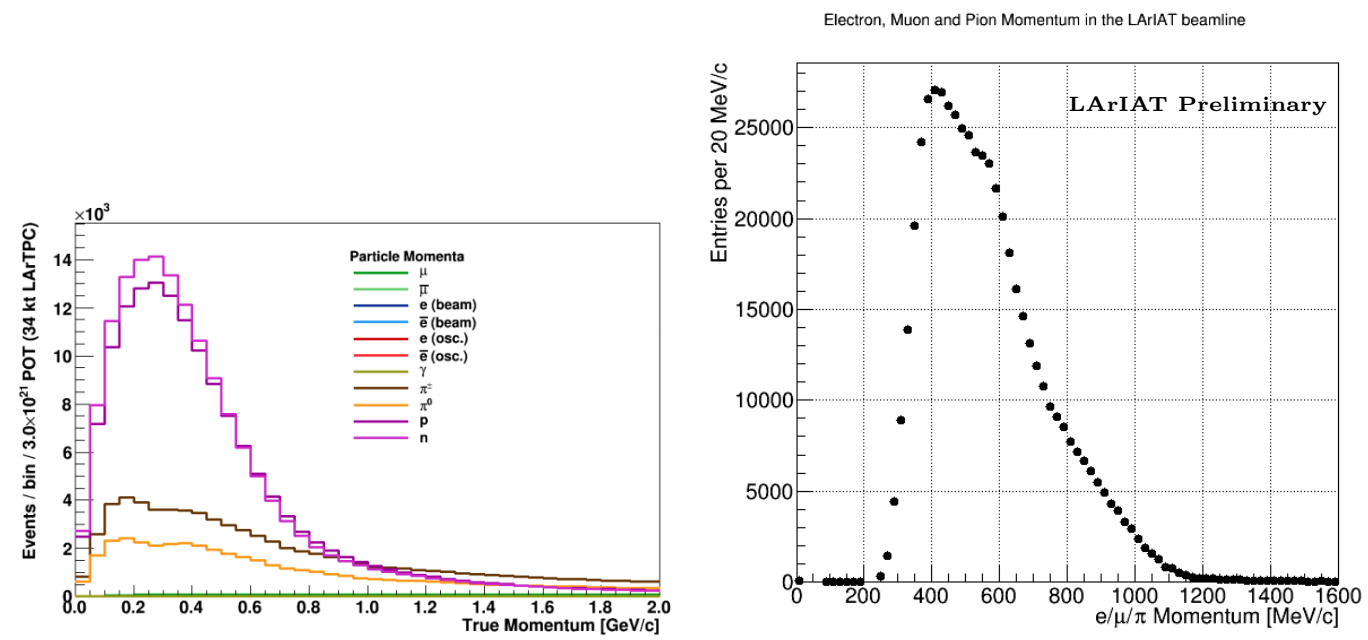

Figure 2.8: Left. Simulation of the products of a $\nu_{e} \mathrm{CC}$ interaction in DUNE, both in particles type and momentum.

Right. Momentum spectrum for low mass particles $(e, \mu, \pi)$ in the LArIAT beamline, negative tune, Run II, Picky Tracks see section 3.2.2. 

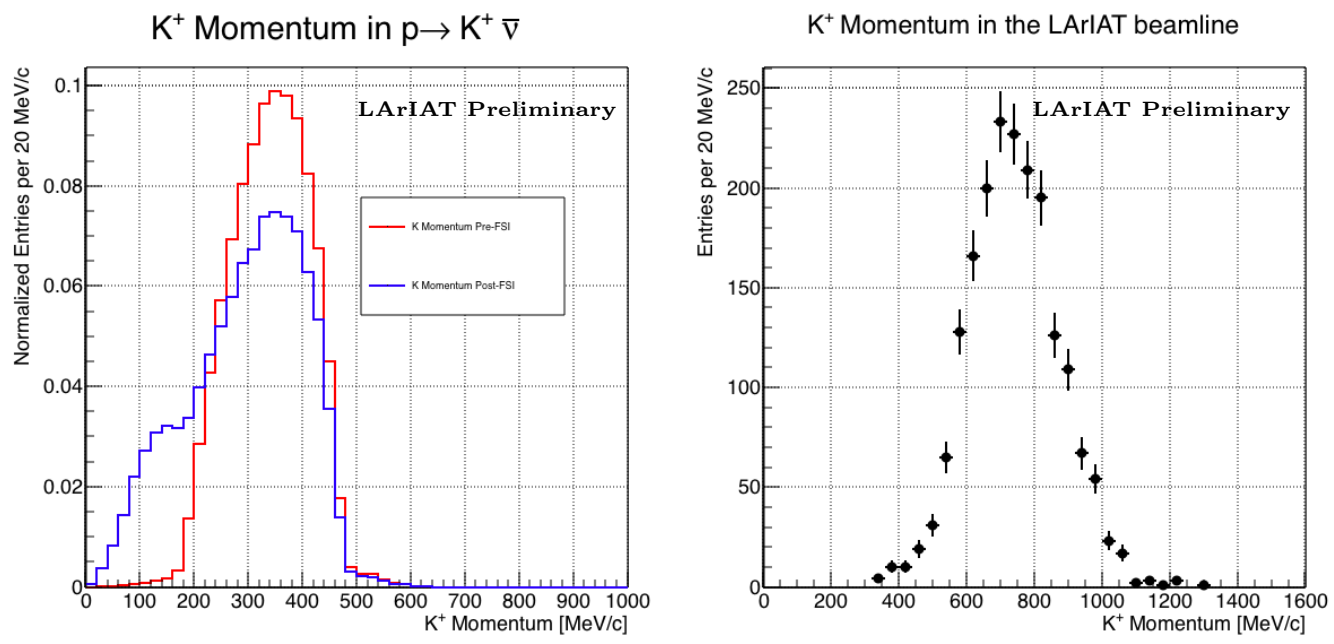

Figure 2.9: Left. Momentum of the kaon outgoing a proton decay $p \rightarrow K^{+} \bar{\nu}$ event as simulated by the Genie 2.8.10 event generator in argon. The red line represents the kaon momentum distribution before undergoing the simulated final state interaction inside the argon nucleus, while the blue line represents the momentum distribution after FSI.

Right. Positive Kaon momentum spectrum in the LArIAT beamline, positive tune, Run II, Picky Tracks see section 3.2.2. 


\section{Chapter 3}

\section{LArIAT: Liquid Argon In A}

\section{Testbeam}

"But, hey we need to be somewhat foolish..."

- Agnes Obel, 2010 -

In this chapter, we describe the LArIAT experimental setup. We start by illustrating the journey of the charged particles in the Fermilab accelerator complex, from the gaseous thermal hydrogen at the Fermilab ion source to the delivery of the LArIAT tertiary beam at MC7. We then describe the LArIAT beamline detectors, the LArTPC, the DAQ and the monitoring system.

\subsection{The Particles' Path to LArIAT}

LArIAT's particle history begins in the Fermilab accelerator complex with a beam of protons. The process of proton acceleration develops in gradual stages (see picture 3.1): gaseous hydrogen is ionized in order to form $\mathrm{H}^{-}$ions; these ions are boosted to $750 \mathrm{keV}$ by a Cockroft-Walton accelerator and injected into the linear accelerator (Linac) that increases their energy up to $400 \mathrm{MeV}$; then, $\mathrm{H}^{-}$ions pass through a 
carbon foil and lose the two electrons; the resulting protons are then injected into a rapid cycling synchrotron, called the Booster; at this stage, protons reach $8 \mathrm{GeV}$ of energy and are compacted into bunches; the next stage of acceleration is the Main Injector, a synchrotron which accelerates the bunches up to $120 \mathrm{GeV}$; in the Main Injector, several bunches are merged into one and are ready for delivery.

The Fermilab accelerator complex works in supercycles of 60 seconds in duration. A $120 \mathrm{GeV}$ primary proton beam with variable intensity is extracted in four-second "spills" and sent to the Meson Center beam line.

LArIAT's home at Fermilab is the Fermilab Test Beam Facility (FTBF), where the experiment characterizes a beam of charged particles in the Meson Center beam line. At FTBF, the primary beam is focused onto a tungsten target to create LArIAT's secondary beam. The secondary beamline is set such that the composition of the secondary particle beam is mainly positive pions. The momentum peak of the secondary beam was fixed at $64 \mathrm{GeV} / \mathrm{c}$ for the LArIAT data considered in this work, although the beam is tunable in momentum between $8-80 \mathrm{GeV} / \mathrm{c}$; this configuration of the secondary beamline assured a stable beam delivery at the LArIAT experimental hall.

The secondary beam impinges then on a copper target within a steel collimator inside the LArIAT experimental hall (MC7) to create the LArIAT tertiary beam, (shown in Fig. 3.2). The steel collimator selects particles produced with a $13^{\circ}$ production angle. The particles are then bent by roughly $10^{\circ}$ through a pair of dipole magnets. By configuring the field intensity of the magnets we allow the particles of LArIAT's tertiary beam to span a momentum range from 0.2 to $1.4 \mathrm{GeV} / \mathrm{c}$. The polarity of the magnet is also configurable and determines the sign of the beamline particles which are focused on the LArTPC. If the magnet polarity is positive the tertiary beam composition is mostly pions and protons with a small fraction of electrons, muons, and kaons. It is the job of the LArIAT beamline equipment to select the 


\section{Fermilab Accelerator Complex}

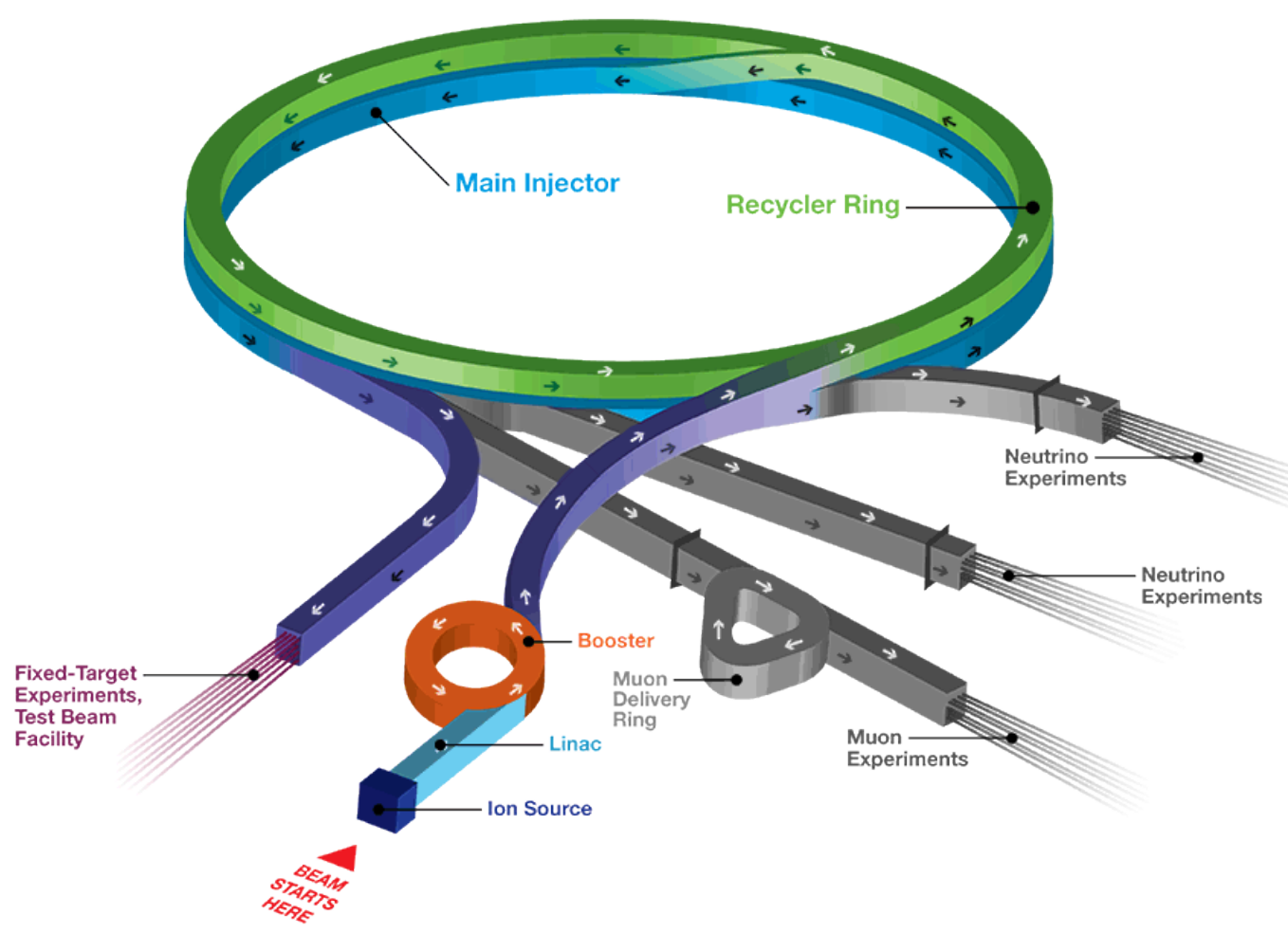

Figure 3.1: Layout of Fermilab Accelerator complex.

particles polarity, to perform particle identification in the beamline and to measure the momentum of the tertiary beam particles before they get to the LArTPC. The LArIAT detectors are described in the following paragraphs.

\subsection{LArIAT Tertiary Beam Instrumentation}

The instrumentation of the LArIAT tertiary beam and the TPC components have changed several times during the three years of LArIAT data taking. The following paragraphs describe the components operational during "Run II", the data taking period relevant to the hadron cross section measurements considered in this thesis.

The key components of the tertiary beamline instrumentation for the hadron cross section analyses are the two bending magnets, a set of four wire chambers (WCs) 


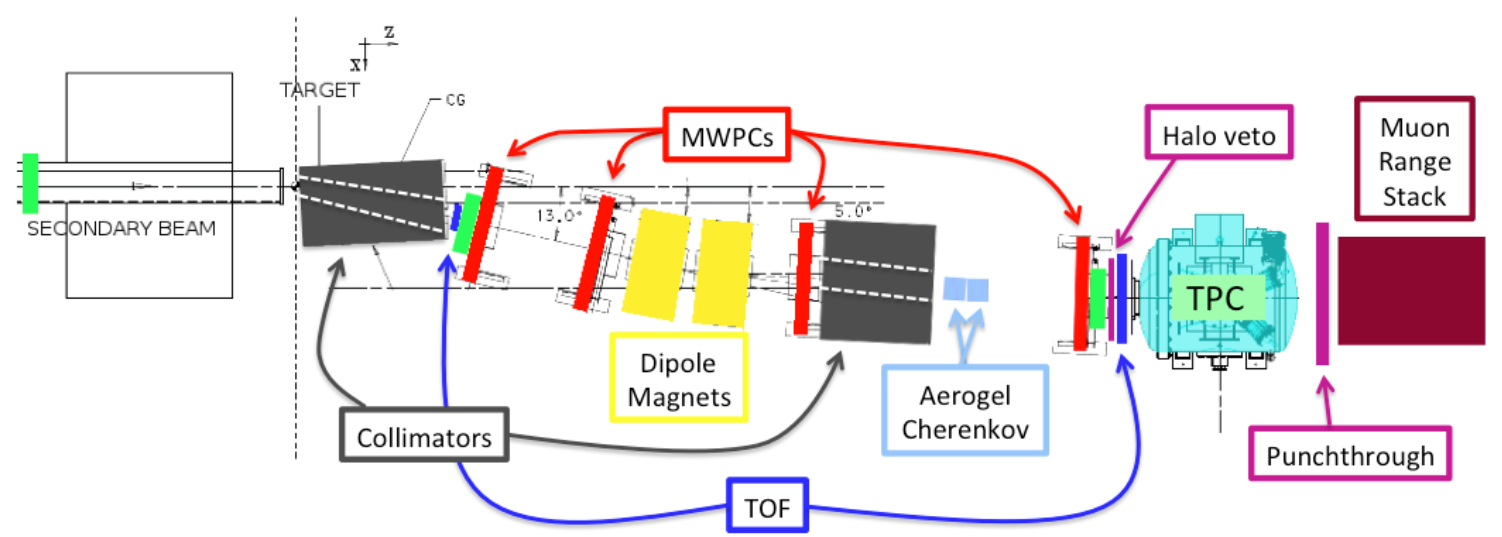

Figure 3.2: Bird's eye view of the LArIAT tertiary beamline. In grey: upstream and downstream collimators; in yellow: bending magnets; in red: multi wire proportional chambers; in blue: time of flight; in green: liquid argon TPC volume; in maroon: muon range statck.

and two time-of-flight scintillating paddles (TOF) and, of course, the LArTPC. The magnets determine the polarity of the particles in the tertiary beam; the combination of magnets and wire chambers determines the particles' momenta, which is used to determine the particle species in conjunction with the TOF. A muon range stack downstream from the TPC and two sets of cosmic paddles configured as a telescope surrounding the TPC are also used for calibration purposes. A couple of Aerogel Cherenkov counters, which we will not describe here as they are not used in the hadron cross section measurements, completes the beamline instrumentation.

\subsubsection{Bending Magnets}

LArIAT uses a pair of identical Fermilab type "NDB" electromagnets, recycled from the Tevatron's anti-proton ring, in a similar configuration used for the MINERvA T977 test beam calibration [61]. The magnets are a fundamental piece of the LArIAT beamline equipment, as they are used for the selection of the particle polarity and for the momentum measurement before the LArTPC. The sign of the current in the magnets allows us to select either positively or negatively charged particles; the value 
of the magnetic field is used in the momentum determination and in the subsequent particle identification.

We describe here the characteristics and response of one magnet, as the second one has a similar response, given its identical shape and history. Each magnet is a box with a rectangular aperture gap in the center to allow for the particle passage. The magnet aperture measures $14.22 \mathrm{~cm}$ in height, $31.75 \mathrm{~cm}$ in width, and $46.67 \mathrm{~cm}$ in length. Since the wire chambers aperture $\left(\sim 12.8 \mathrm{~cm}^{2}\right)$ is smaller than the magnet aperture, only the central part of the magnet gap is utilized. The field is extremely uniform over this limited aperture and was measured with two hall probes, both calibrated with nuclear magnetic resonance probes. The probes measured the excitation curve shown in Figure 3.3 .

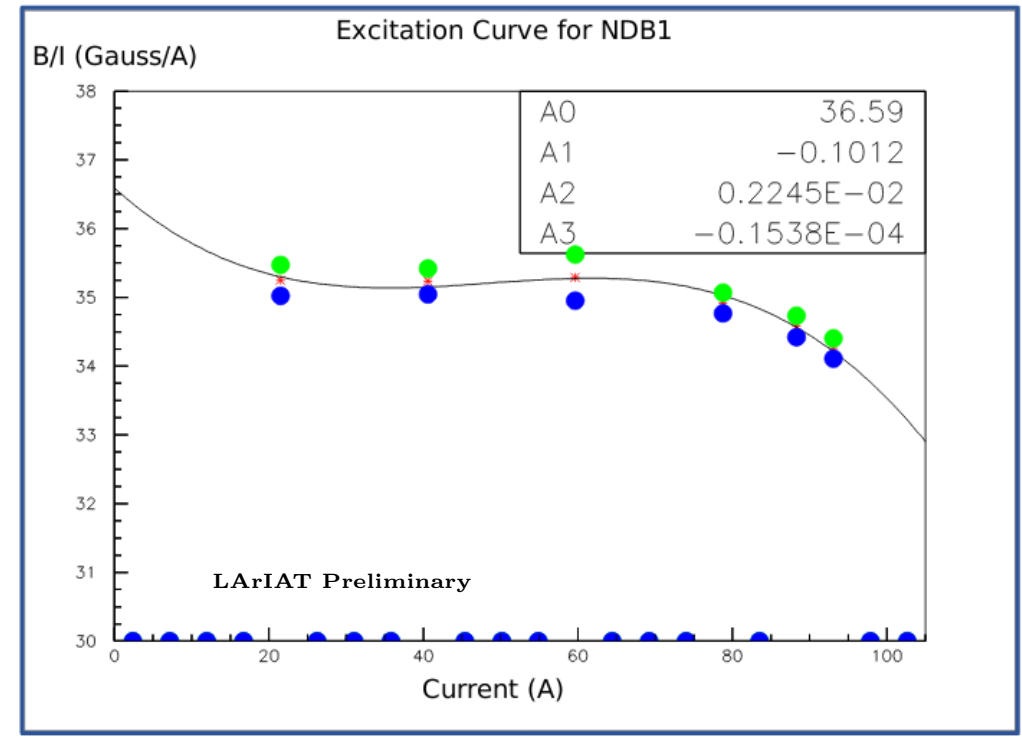

Figure 3.3: Magnetic field over current as a function of the current, for one NDB magnet (excitation curve). The data were collected using two hall probes (blue and green). We fit the readings with a cubic function (black) to average of measurements (red) given in the legend [11].

The current through the magnets at a given time is identical in both magnets. For the Run II data taking period, the current settings explored were $60 \mathrm{~A}$ ( $\mathrm{B} \sim 0.21$ $\mathrm{T})$ and $100 \mathrm{~A}(\mathrm{~B} \sim 0.35 \mathrm{~T})$ in both polarities. Albeit advantageous to enrich the tertiary beam composition with high mass particles such as kaons, we never pushed 
the magnets current over $100 \mathrm{~A}$, so to not overheat them. During operation, we operated an air and water cooling system on the magnets and we remotely monitored the magnet temperatures.

\subsubsection{Multi-Wire Proportional Chambers}

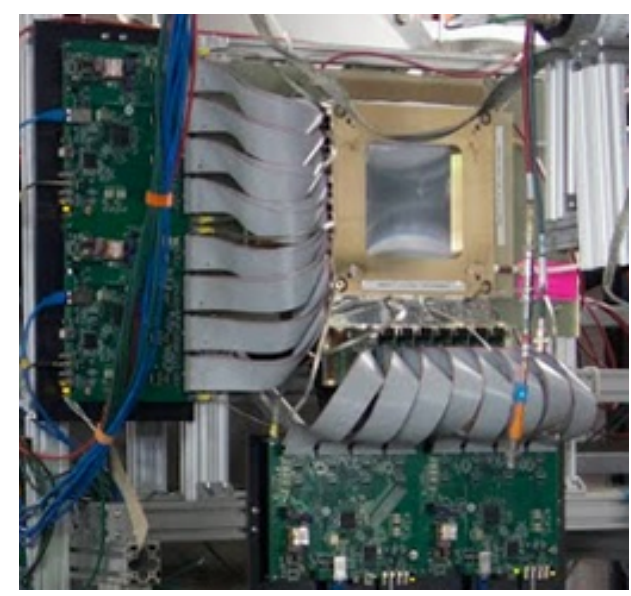

Figure 3.4: One of the four Multi-Wire Proportional Chambers (WC) used in the LArIAT tertiary beamline and related read-out electronics.

LArIAT uses four multi-wire proportional chambers, or wire chambers (WC) for short, two upstream and two downstream from the bending magnets. The geometry of one chamber is shown in Figure 3.4 the WC effective aperture is a square of $12.8 \mathrm{~cm}$ perpendicular to the beam direction. Inside the chamber, the 128 horizontal and 128 vertical wires strung at a distance of $1 \mathrm{~mm}$ from each other in a mixture of $85 \%$ Argon and $15 \%$ isobutane gas. The WC operating voltage is between $2400 \mathrm{~V}$ and $2500 \mathrm{~V}$. The LArIAT wire chambers are an upgraded version of the Fenker Chambers [96, where an extra grounding improves the signal to noise ratio of the electronic readout.

Two ASDQ chips [49] mounted on a mother board plugged into the chamber serve as front end amplifier/discriminator. The chips are connected to a multi-hit TDC [83] which provides a fast OR output used as first level trigger. The TDC time resolution is $1.18 \mathrm{~ns} /$ bin and can accept 2 edges per 9 ns. The maximum event rate acceptable by the chamber system is $1 \mathrm{MHz}$. A full spill of data occurring once per supercycle 
is stored on the TDC board memory at once and read out by a specially designed controller. We use LVDS cables to carry both power and data between the controller and the TDCs and from the controller to the rest of the DAQ.

\section{Multi-Wire Proportional Chambers functionality}

We use the wire chamber system together with the bending magnets to measure the particle's momentum.

In the simplest scenario, only one hit on each and every of the four wire chambers is recorded during a single readout of the detector systems. Thus, we use the hit positions in the two wire chambers upstream of the magnets to form a trajectory before the bend, and the hit positions in the two wire chambers downstream of the magnets to form a trajectory after the bend. We use the angles in the XZ plane between the upstream and downstream trajectories to calculate the $Z$ component of the momentum as follows:

$$
P_{z}=\frac{B_{e f f} L_{e f f}}{3.3\left(\sin \left(\theta_{D S}\right)-\sin \left(\theta_{U S}\right)\right)},
$$

where $B_{\text {eff }}$ is the effective maximum field in a square field approximation, $L_{e f f}$ is the effective length of both magnets (twice the effective length of one magnet), $\theta_{U S}$ is the angle off the $z$ axis of the upstream trajectory, $\theta_{D S}$ is the angle off the $z$ axis of the downstream trajectory and $3.3 c^{-1}$ is the conversion factor from $[\mathrm{T} \cdot \mathrm{m}]$ to $[\mathrm{MeV} / \mathrm{c}]$. By using the hit positions on the third and fourth wire chamber, we estimate the azimuthal and polar angles of the particle trajectory, and we are able to calculate the other components of the momentum.

The presence of multiple hits in a single wire chamber or the absence of hits in one (or more) wire chambers can complicate this simple scenario. The first complication is due to beam pile up, while the latter is due to wire chamber inefficiency. In the case of multiple hits on a single WC, at most one wire chamber track is reconstructed 
per event. Since the magnets bend particles only in the $\mathrm{X}$ direction, we assume the particle trajectory to be roughly constant in the YZ plane, thus we keep the combination of hits which fit best with a straight line. It is still possible to reconstruct the particle's momentum even if the information is missing in either of the two middle wire chambers (WC2 or WC3), by constraining the particle trajectory to cross the plane in between the magnets.

Events satisfying the simplest scenario of one single hit in each of the four wire chambers form the "Picky Track" sample. We construct another, higher statistics sample, where we loosen the requirements on single hit and wire chamber efficiency: the "High Yield" sample. For LArIAT Run II, the High Yield sample is about three times the Picky Tracks statistics. We assume an uncertainty of $2 \%$ for four-point WC track, momentum uncertainty as reported for the same beamline in 61].

\subsubsection{Time-of-Flight System}

Two scintillator paddles, one upstream of the first set of WCs and one downstream of the second set of WCs form LArIAT time-of-flight (TOF) detector system.

The upstream paddle is made of a $10 \times 6 \times 1 \mathrm{~cm}$ scintillator piece, read out by two PMTs mounted on the beam left side which collect the light from light guides mounted on all four edges of the scintillator. The downstream paddle is a $14 \mathrm{x} 14 \mathrm{x}$ $1 \mathrm{~cm}$ scintillator piece read out by two PMTs on the opposite ends of the scintillator, as shown in figure 3.5 . The relatively thin width in the beamline direction minimizes energy loss of beam particles traveling through the scintillator material.

The CAEN 1751 digitizer is used to digitize the TOF PMTs signals at a sampling rate of $1 \mathrm{GHz}$. The 12 bit samples are stored in a circular memory buffer. At trigger time, data from the TOF PMTs are recorded to output in a 28.7 us windows starting approximately $8.4 \mu$ s before the trigger time. 


\section{TOF functionality}

The TOF signals rise time (10-90\%) is $4 \mathrm{~ns}$ and a full width, half-maximum of $9 \mathrm{~ns}$ consistent in time. The signal amplitudes from the upstream TOF and downstream TOF are slightly different: $200 \mathrm{mV}$ for the upstream PMTs but only $50 \mathrm{mV}$ for downstream PMTs. The time of the pulses was calculated utilizing an oversampled template derived from the data itself. We take the pulse pedestal from samples far from the pulse and subtract it from the pulse amplitude. We then vertically stretch a template to match the pedestal-subtracted pulse amplitude and we move

it horizontally to find the time. With this technique, we find a pulse time-pickoff resolution better than 100 ps. The pulse pile up is not a significant problem given the TOF timing resolution and the rate of the particle beam. Leveraging on the pulses width uniformity of any given PMT, we flag events where two pulses overlap as closely in time as 4 ns with a $90 \%$ efficiency according to simulation.

We combine the pulses from the two PMTs on each paddle to determine the particles' arrival time by averaging the time measured from the single PMT, so to minimize errors due to optical path differences in the scintillator. However, a time spread of approximately $300 \mathrm{ps}$ is present in both the upstream and downstream detectors, likely due to transit time jitter in the PMTs themselves.

\subsubsection{Punch-Through and Muon Range Stack Instruments}

The punch-through and the muon range stack (MuRS) detectors are located downstream of the TPC. These detectors provide a sample of TPC crossing tracks without relying on TPC information and can be used to improve particle ID for muons and pions with momentum higher than $450 \mathrm{MeV} / \mathrm{c}$.

The punch-through is simple sheet of scintillator material, read out by two PMTs. The MuRS is a segmented block of steel with four slots instrumented with scintillation bars. The four steel layers in front of each instrumented slot are $2 \mathrm{~cm}, 2 \mathrm{~cm}, 14 \mathrm{~cm}$ 


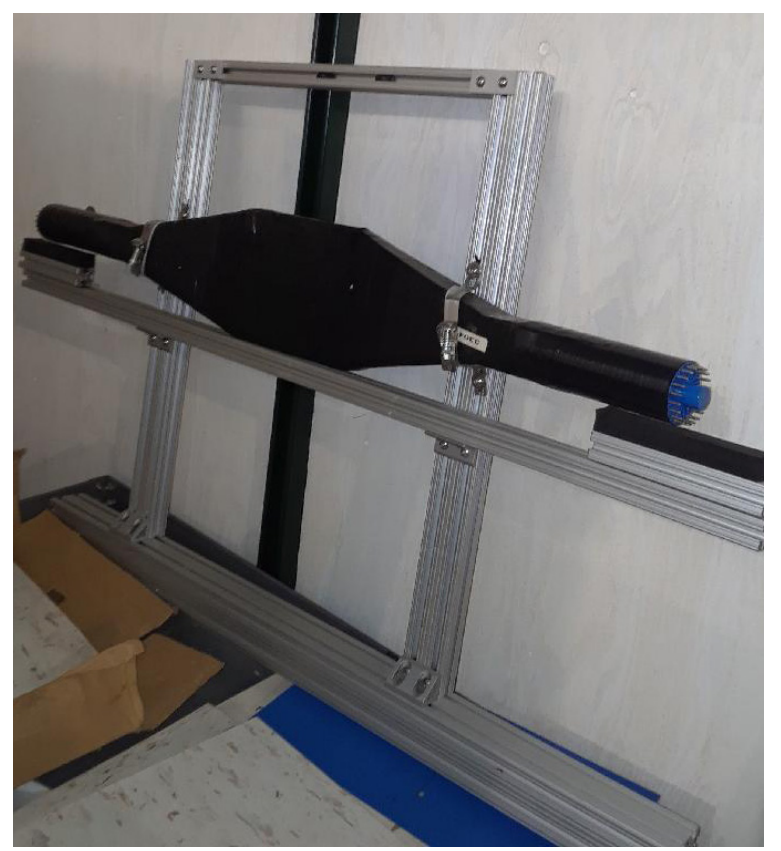

Figure 3.5: Image of the down stream time of flight paddle, PMTs and relative support structure before mounting.

and $16 \mathrm{~cm}$ deep in the beam direction. Each instrumented slot is equipped with four scintillation bars each, positioned horizontally in the direction orthogonal to the beam. Each scintillator is read out by one PMT.

The signals from both the punch-thorough and the MuRS PMTs are sent to a NIM discriminator. If the signal crosses the discriminator threshold, it is digitized in the CAEN V1740, same as the TPC. The sampling time of the CAEN V1740 is slow (of the order of $128 \mathrm{~ns}$ ) and that the pulse shape information from the PMT is lost. A Punch-thorough and MuRS signal will then be simply a "hit" at a given time in the beamline event.

It is worth mentioning here the presence of an additional scintillation paddle between WC4 and the downstream paddle of the TOF system, called halo. The halo is a $39 \times 38 \times 1 \mathrm{~cm}^{3}$ paddle with a $6.5 \mathrm{~cm}$ radius hole in the center, whose original function was to reject beam particles slightly offset from the beamline center. Data from this paddle turned out to be unusable, so our data events include both particle going through the halo scintillation material or through the halo hole. 


\subsubsection{LArIAT Cosmic Ray Paddle Detectors}

LArIAT triggers both on beam events and on cosmic rays events. We perform this latter trigger by using two sets of cosmic ray paddle detectors (a.k.a. "cosmic towers".) The cosmic towers frame the LArIAT cryostat, as one sits in the downstream left corner and the other sits in the upstream right corner of the cryostat. Two paddle sets of four scintillators pieces each make up each cosmic tower, an upper set and a lower set per tower. Of the four paddles, a couple of two matched paddles stands upright while the a second matched pair lies across the top of the assembly in the top sets (or across the bottom of the assembly in the bottom sets). The horizontal couple is used as a veto for particles traveling from inside the TPC out. The four signals from the vertical paddles along one of the body diagonals of the TPC are combined in a logical "AND". This allows to select track due to cosmic muons at the ground level crossing the TPC along one of its diagonals. Cosmic ray muons whose average energy is in the few $\mathrm{GeV}$ range crossing both anode and cathode populate the events triggered this way. This particularly useful sample of tracks can be used for many tasks; for example, we use anode-cathode piercing tracks to cross check

the TPC electric field on data (see Appendix A), to calibrate the charge response of the TPC wires for the full TPC volume and to measure the electron lifetime in the chamber 73.

We retrieved the scintillation paddles from the decommissioning of the CDF detector at Fermilab and we used only the paddles with a counting efficiency greater than $95 \%$ and low noise at working voltage. The measured trigger rate of the whole system is $0.032 \mathrm{~Hz}$, corresponding to $\sim 2$ muons per minute. 


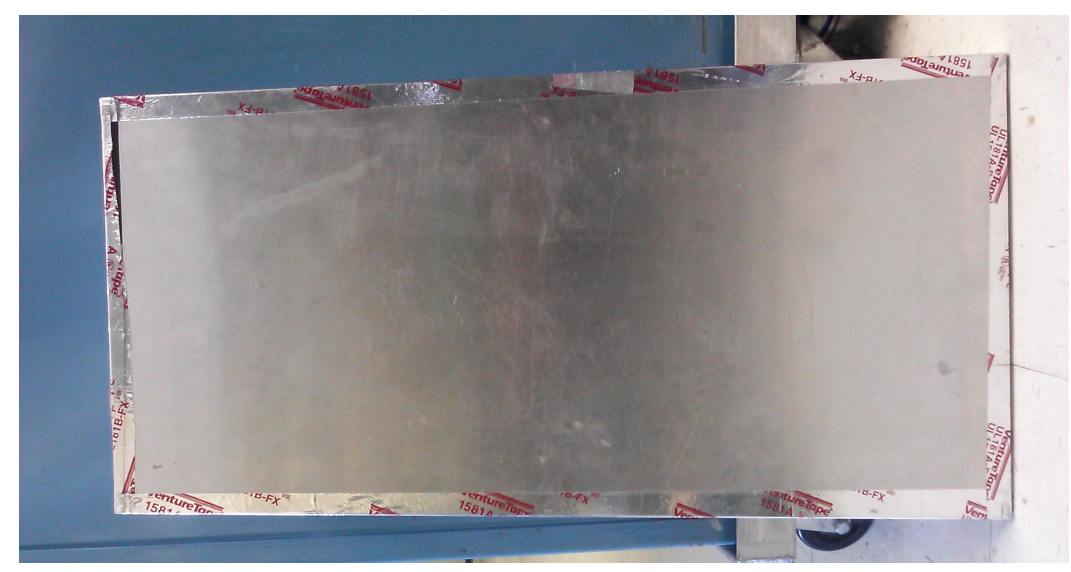

Figure 3.6: Photograph of one of the scintillation counters used in the cosmic towers.

\subsection{In the Cryostat}

The heart of the LArIAT experiment lives in the LArIAT cryostat. In this section, we describe the cryogenic system and the argon purity (Section 3.3.1), the LArIAT TPC (Section 3.3.2 and light collection system 3.3.3.

\subsubsection{Cryogenics and Argon Purity}

LArIAT repurposed the ArgoNeuT cryostat [29] in order to use it in a beam of charged particles, and added a new process piping and a new liquid argon filtration system in FTBF. Inside the LArIAT experimental hall, the cryostat sits in the beam of charged particles with its horizontal main axis oriented parallel to the secondary beam, $3^{\circ}$ off axis from the tertiary beam

Two volumes make up LArIAT cryostat, shown in Figure 3.7 the inner vessel and the outer vessel. Purified liquid argon fills the inner vessel, while the outer volume provides insulation through a vacuum jacket equipped with layers of aluminized mylar superinsulation. The inner vessel is a cylinder of $130 \mathrm{~cm}$ length and $76.2 \mathrm{~cm}$ diameter, containing about $550 \mathrm{~L}$ of LAr, corresponding to a mass of 0.77 ton. We run the signal cables for the LArTPC and the high voltage feedthrough through a "chimney" at the top and mid-length of the cryostat. 

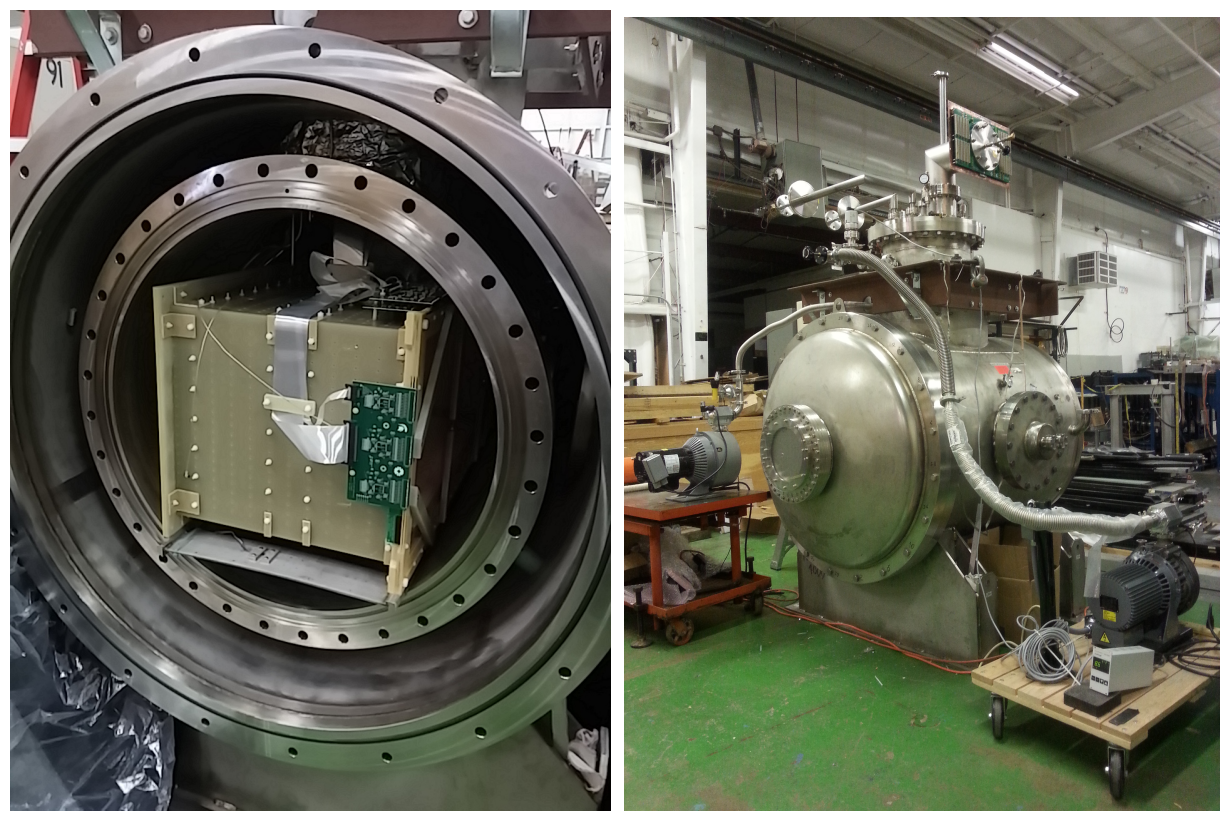

Figure 3.7: Left: the LArIAT TPC in the inner volume of the open cryostat. Right: cryostat fully sealed ready to be transported to FTBF.

Given the different scopes of the ArgoNeuT and LArIAT detectors, we made several modifications to the ArgoNeuT cryostat in order to use it in LArIAT. In particular, the modifications shown in Figure 3.8 were necessary to account for the beam of charged particles entering the TPC and to employ the new FTBF liquid argon purification system. We added a "beam window" on the front outer end cap and an "excluder" on the inner endcap, with the purpose of minimizing the amount of non-instrumented material upstream of the TPC's active volume. The amount of noninstrumented material in front of the TPC for LArIAT corresponds to $\sim 0.3$ electron radiation lengths $\left(X_{0}\right)$, to compare against the $\sim 1.6 X_{0}$ of ArgoNeuT. To allow studies of the scintillation light, we added a side port feedthrough which enables the mounting of the light collection system, as well as the connections for the corresponding signal and high-voltage cables (see Section 3.3.3). We modified the bottom of the cryostat adding Conflat and ISO flange sealing to connect the liquid argon transfer line to the new argon cooling and purification system.

As in any other LArTPC, argon purity is a crucial parameter for LArIAT. Indeed, 

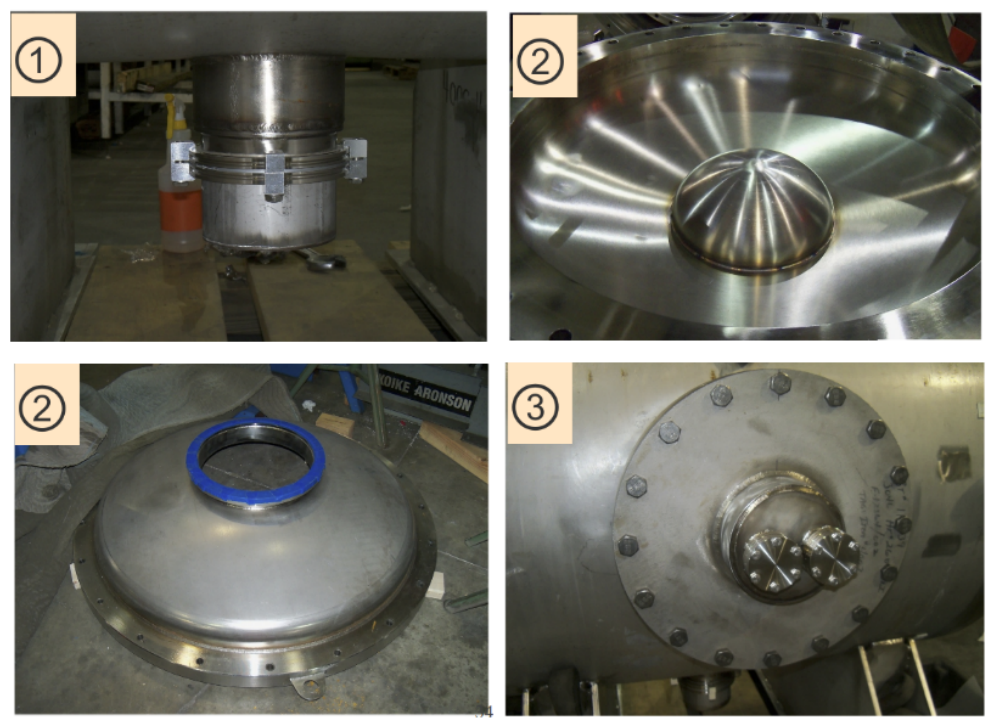

Figure 3.8: Main modifications to the ArgoNeuT cryostat: 1) outlet for connection to the purification system at the bottom of the cryostat; 2) the "beam-window" on the outer endcap and "excluder" which reduces the amount of non-instrumented material before the TPC; 3 ) the side port to host the light collection system.

the presence of contaminants affects both the basic working principles of a LArTPC, as shown in Section 2.1.2 electronegative contaminants such as oxygen and water decrease the number of ionization electrons collected on the wires after drifting through the volume. In addition, contaminants such as Nitrogen decrease the light yield from scintillation light, especially in its slow component. In LArIAT, contaminations should not exceed the level of 0.2 parts per billion (ppb). We achieve this level of purity in several stages. The specifics required for the commercial argon bought for LArIAT are 2 parts per million (ppm) oxygen, 3.5 ppm water, and 10 ppm nitrogen. This argon is monitored with the use of commercial gas analyzer. Argon is stored in a dewar external to LArIAT hall and filtered before filling the TPC. LArIAT uses a filtration system designed for the Liquid Argon Purity Demonstrator (LAPD) [62]: half of a 77 liter filter contains a $4 \mathrm{~A}$ molecular sieve able to remove mainly water, while the other half contains BASF CU-0226 S, a highly dispersed copper oxide impregnated on a high surface area alumina, apt to remove mainly oxygen. A single pass of argon 
in the filter is sufficient to achieve the necessary purity, unless the filter is saturated. In case the filter saturates, the media needs to be regenerated by using heated gas; this happened twice during the Run II period ${ }^{1}$. The electron lifetime during the full LArIAT data taking are shown in Figure 2.4. The filtered argon reaches the inner vessel via a liquid feedthrough which is routed to the bottom of the cryostat. Argon is not recirculated in the system; rather, it boils off and vents to the atmosphere. During data taking, we replenish the argon in the cryostat every 6 hours to keep the TPC high voltage feedthrough and cold electronics always submerged. In fact, we constantly monitor the level, temperature, and pressure of the argon both in the commercial dewar and inside the cryostat during data taking.

\subsubsection{LArTPC: Charge Collection}

The LArIAT Liquid Argon Time Projection Chamber is a rectangular box of dimensions $47 \mathrm{~cm}$ (drift) x $40 \mathrm{~cm}$ (height) x $90 \mathrm{~cm}$ (length), containing 170 liters of Liquid Argon. The LArTPC three major subcomponents are

1) the cathode and field cage,

2) the wire planes,

3) the read-out electronics.

\section{Cathode and field cage}

A G10 plain sheet with copper metallization on one of the 40 x $90 \mathrm{~cm}$ inner surfaces forms the cathode. A high-voltage feedthrough on the top of the LArIAT cryostat delivers the high voltage to the cathode; the purpose of the high voltage system (Figure 3.9) is to drift ionization electrons from the interaction of charged particles

1. We deemed the filter regeneration necessary every time the electron lifetime dropped under 100 us. 
in the liquid argon to the wire planes. The power supply used in this system is a Glassman LX125N16 99] capable of generating up to $-125 \mathrm{kV}$ and $16 \mathrm{~mA}$ of current, but operated at $-23.5 \mathrm{kV}$ during LArIAT Run-II. The power supply is connected via high voltage cables to a series of filter pots before finally reaching the cathode.

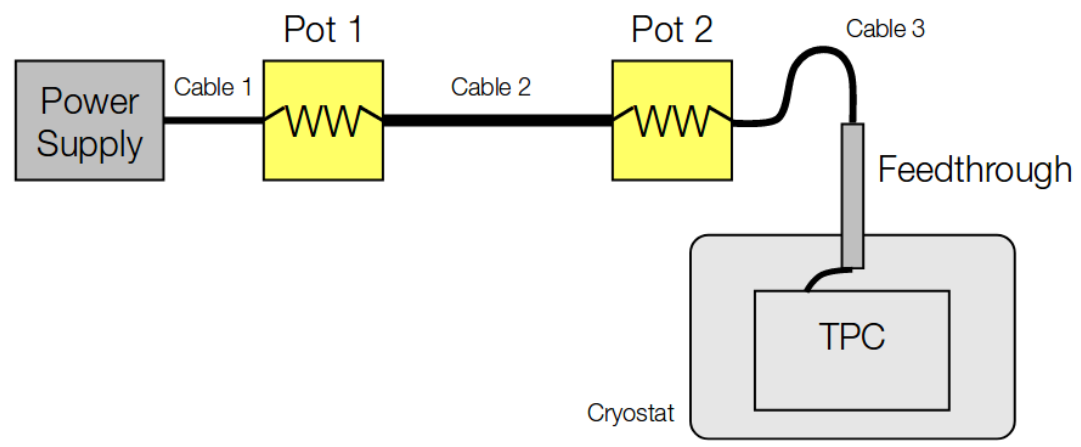

Figure 3.9: Schematic of the LArIAT high voltage system.

The field cage is made of twenty-three parallel copper rings framing the inner walls of the G10 TPC structure. A network of voltage-dividing resistors connected to the field cage rings steps down the high voltage from the cathode to form a uniform electric field. The electric field over the entire TPC drift volume is $486 \mathrm{~V} / \mathrm{cm}$, as measured in appendix A. The maximum drift length, i.e. the distance between cathode and anode planes, is $47 \mathrm{~cm}$.

\section{Wire planes}

LArIAT Run-II has three wire planes separated by $4 \mathrm{~mm}$ spaces: in order of increasing distance from the cathode, they are the shield, the induction and the collection plane. The "wire pitch", i.e., the distance between two adjacent wires in a given plane, is $4 \mathrm{~mm}$. The shield plane counts 225 parallel wires of equal length oriented vertically. This plane is not connected with the read-out electronics; rather it shields the outer planes from extremely long induction signals due to the ionization in the whole drift volume. As the shield plane acts almost like a Faraday cage, the resulting shape of 
signals in the first instrumented plane (induction) is easier to reconstruct. Both the induction and collection planes count 240 parallel wires of different length oriented at $60^{\circ}$ from the vertical with opposite signs. Electrons moving past the induction plane will induce a bipolar pulse on its wires; the drifting electrons will be then collected on the collection plane's wires, forming a unipolar pulse.

The three wire planes and the cathode form three drift volumes, as shown in Figure 3.10. The main drift volume is defined as the region between the cathode plane and the shield plane (C-S). The other two drift regions are those between the shield plane and the induction plane (S-I), and between the induction plane and the collection plane (I-C). The electric field in these regions is chosen to satisfy the charge transparency condition and allow for 100\% transmission of the drifting electrons through the shield and the induction planes; for example, the transparency condition between for the shield plane reads

$$
\frac{E_{S-I}}{E_{C-S}}>\frac{1+\rho}{1-\rho}
$$

where $E_{C-S}$ is the electric field in the region between the cathode and the shield plane, $E_{S-I}$ is the electric field in the region between the shield and the induction plane, and $\rho=\frac{2 \pi r}{D}$ is a factor accounting for the radius of the wires $r(152 \mu m)$ and the gap distance $D(4 \mathrm{~mm})$. An analogous condition can be written for the induction plane.

Table 3.1 provides the default voltages applied to the cathode and the shield, induction, and collection plane.

Table 3.1: Cathode and anode planes default voltages

\begin{tabular}{|l|l|l|l|}
\hline Cathode & Shield & Induction & Collection \\
\hline$-23.17 \mathrm{kV}$ & $-298.8 \mathrm{~V}$ & $-18.5 \mathrm{~V}$ & $338.5 \mathrm{~V}$ \\
\hline
\end{tabular}




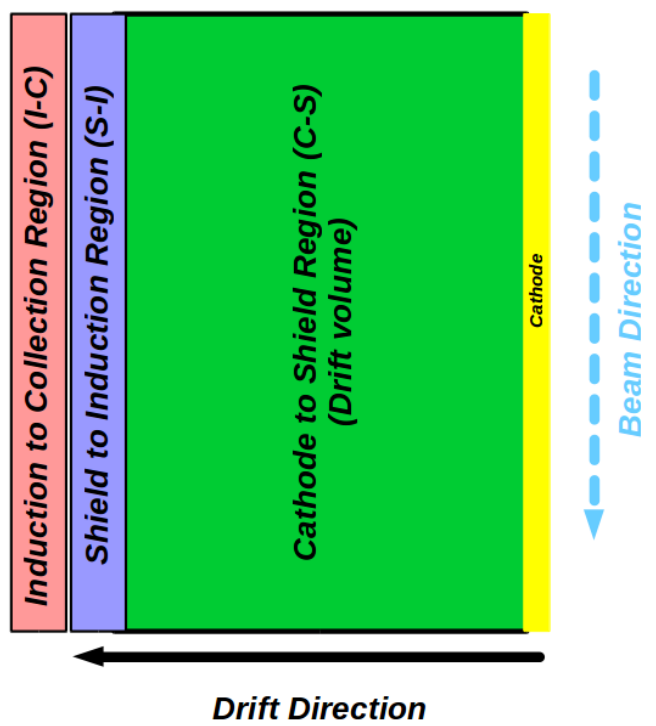

Figure 3.10: Schematic of the three drift regions inside the LArIAT TPC: the main drift volume between the cathode and the shield plane (C-S) in green, the region between the shield plane and the induction plane (S-I) in purple, and the region between the induction plane and the collection plane (I-C) in pink.

\section{Electronics}

Dedicated electronics read the induction and collection plane wires, for a total of 480-channel analog signal path from the TPC wires to the signal digitizers. A digital control system for the TPC-mounted electronics, a power supply, and a distribution system complete the front-end system. Figure 3.11 shows a block diagram of the overall system. The direct readout of the ionization electrons in liquid argon forms typically small signals on the wires, which need amplification in oder to be processed. LArIAT performs the amplification stage directly in cold with amplifiers mounted on the TPC frame inside the liquid argon. The BNL ASICs adopted in LArIAT are designated as LArASIC, version 4-star and are the same used by the MicroBooNE experiment $[76]$. The signal from the ASICs are driven to the other end of the readout chain, to the CAEN V1740 digitizers [6]. The CAEN V1740 has a 12 bit resolution and a maximum input range of $2 \mathrm{VDC}$, resulting in about 180 ADC count for a crossing MIP. 


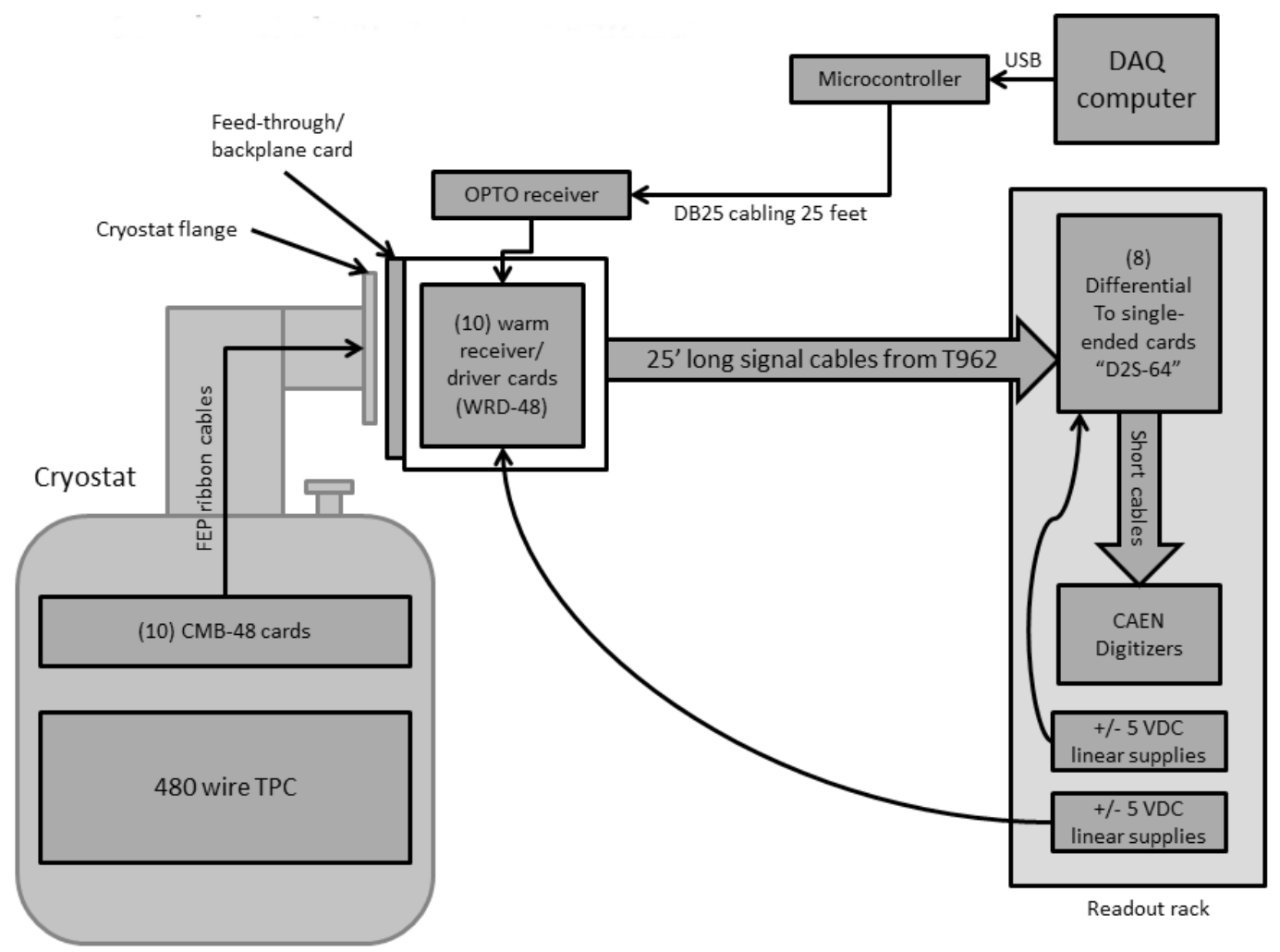

Figure 3.11: Overview of LArIAT Front End electronics. 

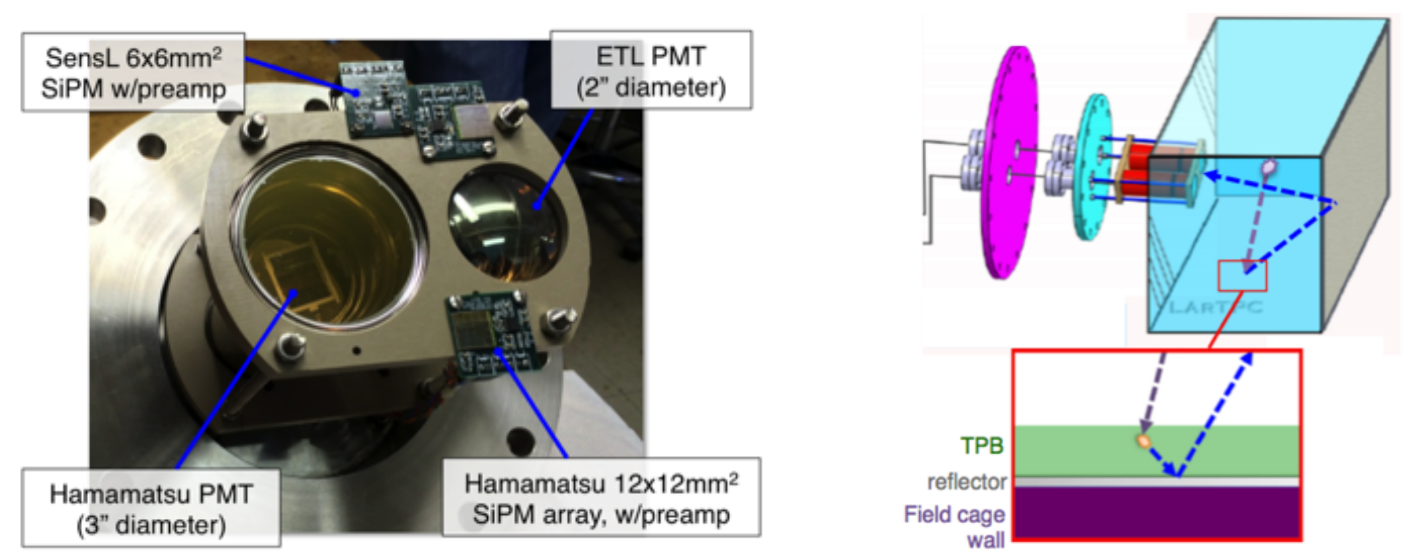

Figure 3.12: LArIAT's photodetector system for observing LAr scintillation light inside the TPC (left), and a simplified schematic of VUV light being wavelengthshifting along the TPB-coated reflecting foils (right).

\subsubsection{LArTPC: Light Collection}

The collection of scintillation photons is the second mechanism of particle detection in argon other than the ionization electrons. Over the course of LArIAT's three years of data taking, the light collection system changed several times. We describe here the light collection system for Run II. Two PMTs, a 3-inch diameter Hamamatsu R-11065 and 2-inch diameter ETL D757KFL [71], as well as three SiPMs arrays (two Hamamatsu S11828-3344M 4x4 arrays and one single-channel SensL MicroFB-60035) are mounted on the PEEK support structure. PEEK screws into an access flange as shown in Figure 3.12 , on the anode side, leaving approximately $5 \mathrm{~cm}$ of clearance from the collection plane.

Liquid argon scintillates in vacuum-ultraviolet (VUV) range at $128 \mathrm{~nm}$; since cryogenic PMTs are not sensitive to VUV wavelengths, we need to shift the light to a range that is visible to the PMTs. In LArIAT, the wavelength shifting is achieved by installing highly-reflective 3M VIKUITI dielectric substrate foils coated with a thin layer of tetraphenyl-butadiene (TPB) on the four unbiased walls of the TPC. The scintillation light interaction with the TPB emits one or more visible photons, which are then reflected into the chamber. Thus, the light yield increases and results in 
higher uniformity of light across the TPC active volume, allowing the possibility of light-based calorimetry, currently under study.

For Run II, we coated the windows of the ETL PMT and the SensL SiPM with a thin layer of TPB. In doing so, some of the VUV scintillation light converts into visible right at the sensor faces, keeping information on the direction of the light source. Information about the light directionality is hindered for the light reflected on foils, as the reflection is uniform in angle.

\subsection{Trigger and DAQ}

The LArIAT DAQ and trigger system governs the read out of all the many subsystems forming LArIAT. The CAEN V1495 module [5] and its user-programmable FPGA are the core of this system. Every $10 \mathrm{~ns}$, this module checks for matches between sixteen logical inputs and user-defined patterns in the trigger menu; if it finds a match for two consecutive clock ticks, that trigger fires.

LArIAT receives three logic signals from the Fermilab accelerator complex related to the beam timing which we use as input triggers: a pulse just before the beam, a pulse indicating beam-on, and a beam-off pulse.

The beam instruments, the cosmic ray taggers, and the light collection system provide the other NIM-standard logic pulse inputs to the trigger decision. We automatically log the trigger inputs configuration with the rest of the DAQ configuration at the beginning of each run.

Fundamental inputs to the trigger card come from the TOF (see Section 3.2.3) and the wire chambers (see Section 3.2.2), as activity in these systems points to the presence of a charged particle in tertiary beam line. In particular, the discriminated pulses from the TOF PMTs form a NIM logic pulse for the trigger logic. We ask for a coincidence within a $20 \mathrm{~ns}$ window for all the pulses from the PMTs looking at 
the same scintillator block and use a delayed coincidence between the upstream and downstream paddle to inform the trigger decision. In order to form a coincidence between the upstream and downstream paddles, we delay the upstream paddle coincidence by $20 \mathrm{~ns}$ and widen it by $100 \mathrm{~ns}$. The delay and widening are necessary to account for both lightspeed particles and slower particles (high-mass) to travel the $6.5 \mathrm{~m}$ between the upstream and the downstream paddles. For the read out of the wire chambers, we use a total of sixteen multi-hit TDCs [83], four per chamber: two TDC per plane (horizontal and vertical), sixty-four wires per TDC. In each TDC, we keep the logical "OR" for any signal over threshold from the sixty-four wires. We then require a coincidence between the "OR" for the horizontal TDCs and the "OR" for the vertical TDCs: with this logic we make sure that at least one horizontal wire and one vertical wire saw significant signal in one wire chamber. The single logical pulse from each of the four wire chambers feeds into the first four inputs to the V1495 trigger card. We require a coincidence within $20 \mathrm{~ns}$ of at least three logical inputs to form a trigger.

The cosmic towers (see Section 3.2.5) provide another primary input to the trigger, in order to capture long tracks from cosmic muons crossing the TPC. We use NIM modules to require coincidences between one upper and one lower paddle set of any opposite cosmic towers. The OR all the opposite towers' coincidences is fed as an input to the trigger card.

We use the signal from the cryogenic PMTs (see Section 3.3.3) to form several interesting triggers. The coincidence of signals from all the PMT pulses within $\sim 20 \mathrm{~ns}$ is an indication of ionizing radiation in the TPC and forms a trigger input. The coincidence of two subsequent scintillation logic pulses delayed by a maximum of $7 \mu \mathrm{s}$ forms the Michel electron trigger. 


\subsection{Control Systems}

LArIAT is a complex ensemble of systems which needed to be monitored simultaneously during data taking. We performed the monitoring of the systems operations with a slow control system, a DAQ monitoring system and a low level data quality monitoring system described in the following sections.

\section{Slow Control}

We used the Synoptic Java Web Start framework [21] as a real-time display of subsystem conditions. Synoptic provides a Graphical User Interface that talks to the Fermilab Accelerator Control System via the ACNET protocol. Its simple GUI allowed us to change the operating parameters and to graph the trends of several variables of interest for all of the tertiary beam detectors. Among the most important quantities monitored by Synoptic are the level of argon in both the inner vessel and the external dewar, the operating voltages of cathode and wire planes, of the PMTs and SiPMs, and of the four wire chambers, as well as the magnet temperatures. Figure 3.13 shows an example of the monitoring system. LArIAT uses the Accelerator Control NETwork system (ACNET) to monitor the beam conditions of the MCenter beamline. For example, the horizontal and vertical position of the beam at the first two wire chambers (WC1 and $\mathrm{WC} 2$ ) are shown in 3.14 as seen by the shifter during data taking.

\section{DAQ Monitoring}

We monitor the data taking and the run time evolution with the Run Status Webpage (http://lariat-wbm.fnal.gov/lariat/run.html), a webpage updated in real-time. The page displays, among other information, the total number of triggers in the event, the total number of detectors triggered during a beam spill, the trigger patterns, the number of times a particular trigger pattern was satisfied during a beam spill, and 


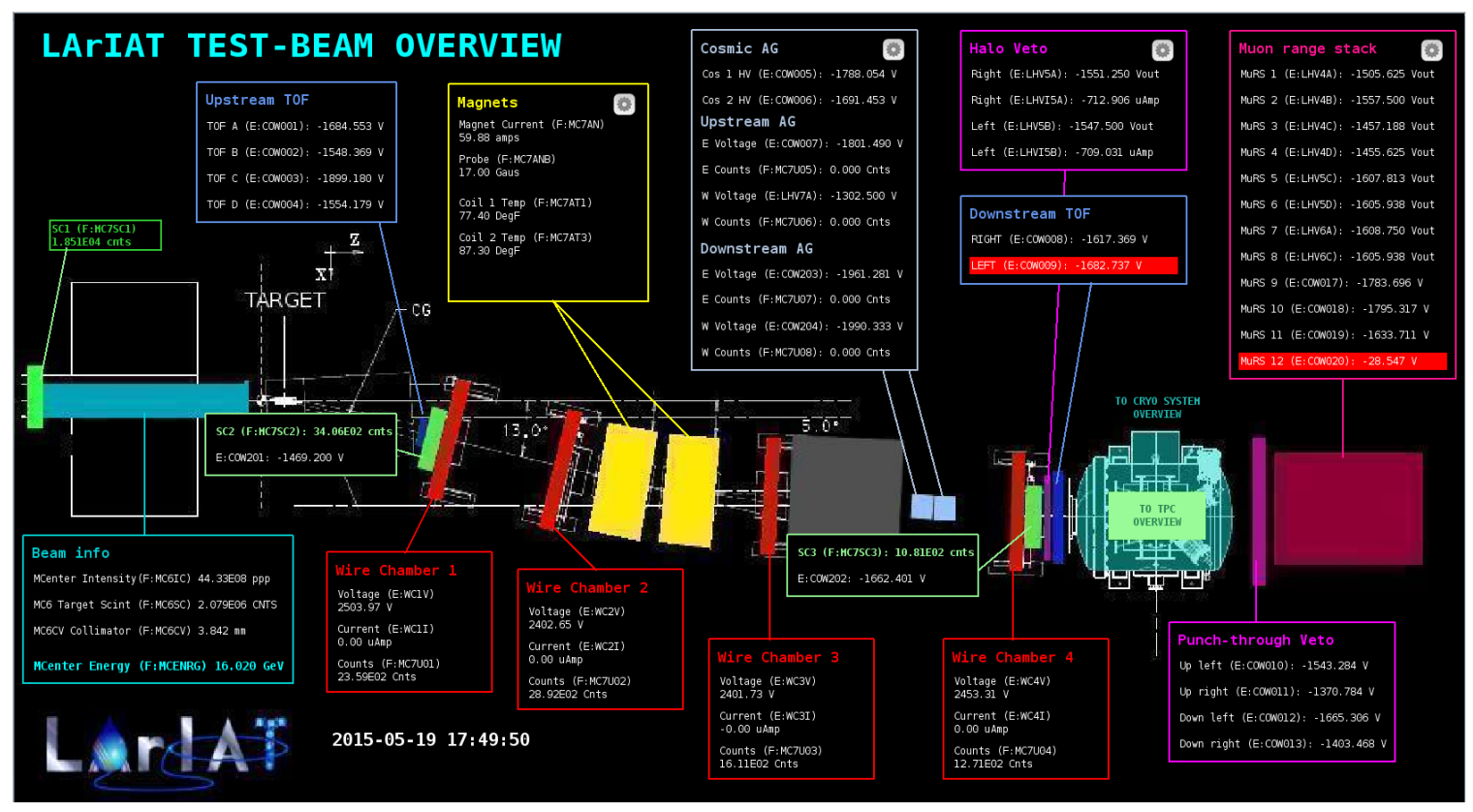

Figure 3.13: Interface of the Synoptic slow control system

the current time relative to the Fermilab accelerator complex supercycle. A screen shot of the page is show in figure 3.15 .

\section{Data Quality Monitoring}

We employ two systems to ensure the quality of our data during data taking: the Near-Real-Time Data Quality Monitoring and the Event Viewer.

The Near-Real-Time Data Quality Monitoring (DQM) is a webpage which receives updates from all the VME boards in the trigger system and displays the results of a quick analysis of the DAQ stream of raw data on a spill-by-spill basis. The DQM allows the shifter to monitor almost in real time (typically with a 2-minute delay) a series of low level-quantities and compare them to past collections of beam spills. Some of the variables monitored in the DQM are the pedestal mean and RMS on CAEN digitizer boards of the TPC wires and PMTs of the beamline detectors, the hit occupancy and timing plots on the wire chambers, and number of data fragments recorded that are used to build a TPC event. Abnormal values for low-level quantity 


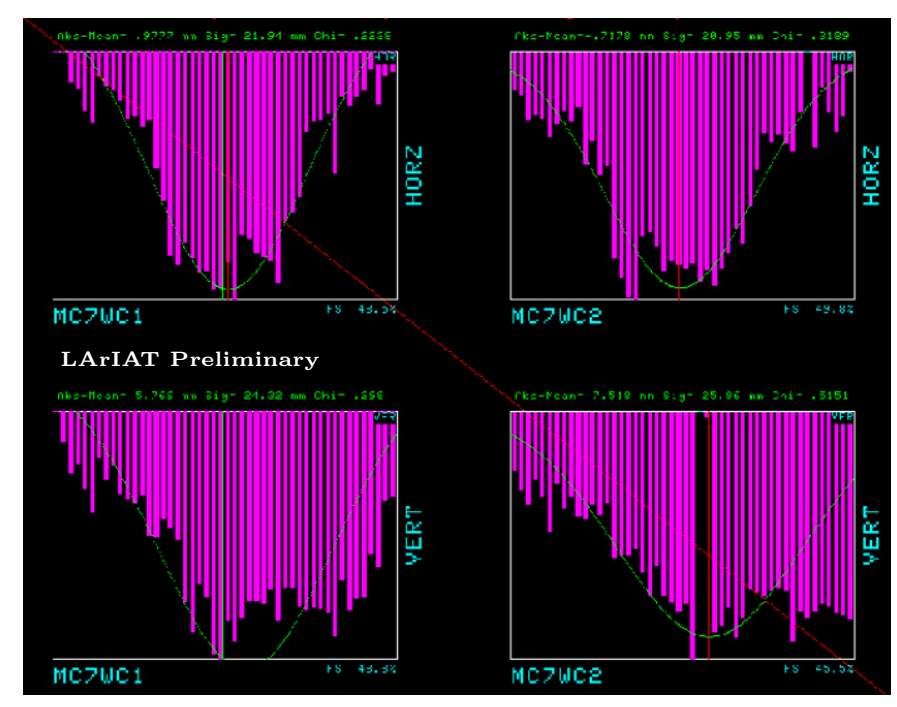

Figure 3.14: Beam position at the upstream wire chambers monitored with ACNET. in the data activates a series of alarms in the DQM; this quick feedback on the DAQ and beam conditions is fundamental to assure a fast debugging of the detector and a very efficient data taking during beam uptime.

The online Event Viewer displays a two dimensional representation (Wire vs Time) of LArIAT TPC events on both the Induction and the Collection planes in near real time. The raw pulses collected by the DAQ on each wire are plotted as a function of drift time, resulting in an image of the TPC event easily readable by the shifter. This tool guarantees a particularly good check of the TPC operation which activate an immediate feedback for troubleshooting a number of issues. For example, it is easy for the shifter to spot high occupancy events and request a reduction of the primary beam intensity, or to spot a decrease of the argon purity which requires the regeneration of filters, or to catch the presence of electronic noise and reboot the ASICs. An example of high occupancy event is shown in 3.16 . 


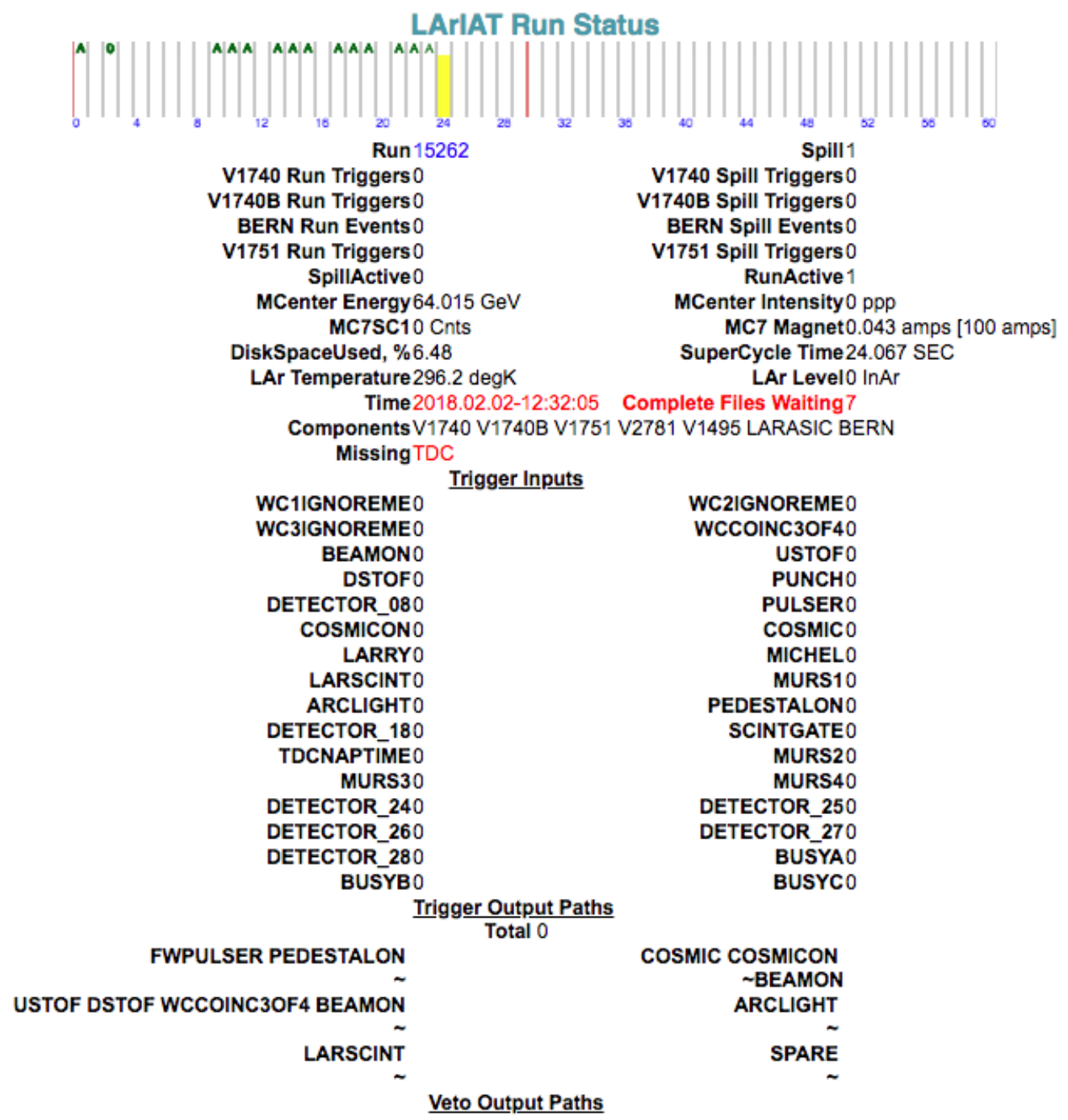

Figure 3.15: Run Status page at LArIAT downtime. At the top the yellow bar displays the current position in the Fermilab supercycle. Interesting information to be monitored by the shifter were the run number and number of spills, time elapsed from data taking (here in red), the energy of the secondary beam and the trigger paths. 


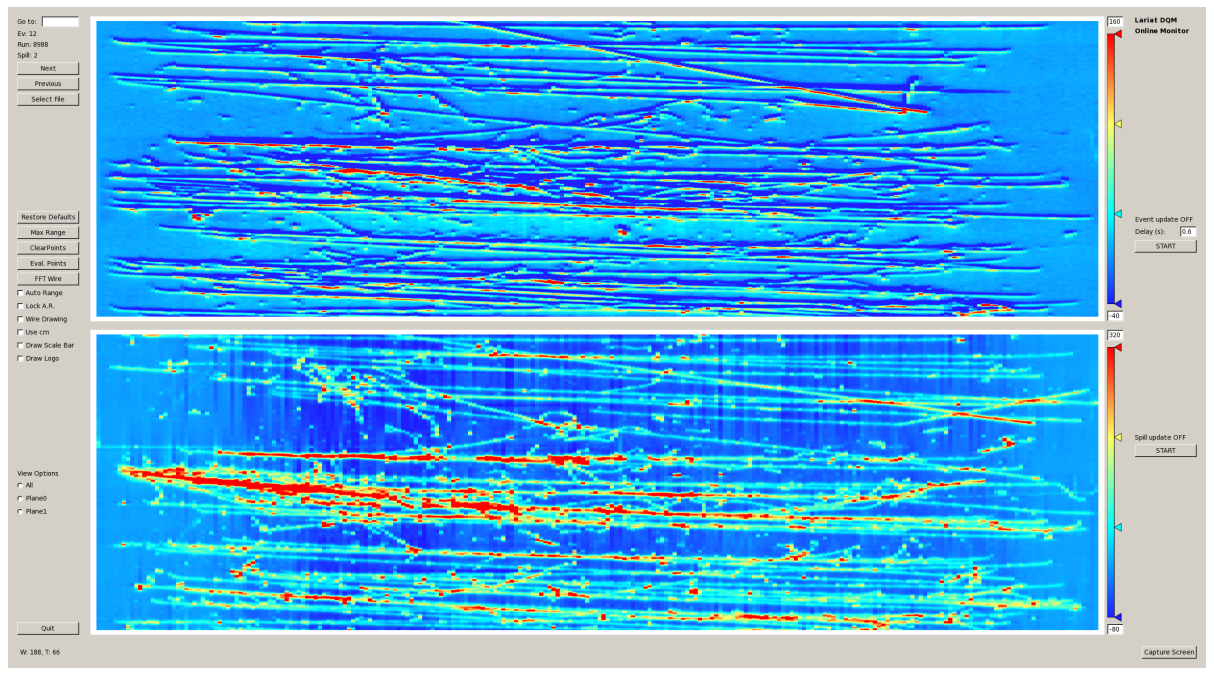

Figure 3.16: High occupancy event display: induction plane (top) and collection plane (bottom). 


\section{Chapter 4}

\section{Total Hadronic Cross Section Measurement Methodology}

"Like a lemon to the lime and the bubble to the bee"

- Eazy-E, 1993 -

This chapter describes the general procedure employed to measure total hadronic interaction cross sections on argon in LArIAT. Albeit with small differences, both the $\left(\pi^{-}, \mathrm{Ar}\right)$ and $\left(\mathrm{K}^{+}, \mathrm{Ar}\right)$ total hadronic cross section measurements rely on the same procedure. We start by selecting the particle of interest using a combination of beamline detectors and TPC information (Section 4.1). We then perform a handshake between the beamline information and the TPC tracking to assure the selection of the correct TPC track (Section 4.2) associated to the corresponding beam particle. We then apply the "thin slice" method to measure the "raw" hadronic cross section (Section 4.3). A series of corrections are then evaluated and applied to obtain the final cross section (Section 4.3.3.

At the end of this chapter, we show a sanity check of the methodology by applying the thin slice method employing only MC truth information and retrieving the expected MC cross section for pions and kaons (Section 4.4). 


\subsection{Event Selection}

The measurement of the $\left(\pi^{-}, \mathrm{Ar}\right)$ and $\left(\mathrm{K}^{+}, \mathrm{Ar}\right)$ total hadronic cross section in LArIAT starts by selecting the pool of pion or kaon candidates and measuring their momentum before they enter the LAr volume. This is done through the series of selections on beamline and TPC information described in the next sections. The summary of the event selection in data is reported in Table 4.1 .

\subsubsection{Selection of Beamline Events}

We leverage the beamline particle identification and momentum measurement before entering the TPC as an input to evaluate the kinetic energy for the hadrons used in the cross sections measurements. To this end, we select the LArIAT data to keep only events whose wire chamber and time of flight information is registered (line 1 in in Table 4.1). Additionally, we perform a check of the plausibility of the trajectory inside the beamline detectors: given the position of the hits in the four wire chambers, we make sure the particle's trajectory does not cross any impenetrable material such as the collimator and the magnets steel (line 2 in in Table 4.1).

\begin{tabular}{|l|c|c|}
\hline & Run-II Neg Pol & Run-II Pos Pol \\
\hline 1. Events Reconstructed in Beamline & 158396 & 260810 \\
\hline 2. Events with Plausible Trajectory & 147468 & 240954 \\
\hline 3. Beamline $\pi^{-} / \mu^{-} / e^{-}$Candidate & 138481 & N.A. \\
\hline 4. Beamline $K^{+}$Candidate & N.A & 2837 \\
\hline 5. Events Surviving Pile Up Filter & 108929 & 2389 \\
\hline 6. Events with WC2TPC Match & 41757 & 1081 \\
\hline 7. Events Surviving Shower Filter & 40841 & N.A. \\
\hline 8. Available Events For Cross Section & 40841 & 1081 \\
\hline
\end{tabular}

Table 4.1: Number of data events for Run-II Negative and Positive polarity 


\subsubsection{Particle Identification in the Beamline}

In data, the main tool to establish the identity of the hadron of interest is the LArIAT tertiary beamline, in its function of mass spectrometer. We combine the measurement of the time of flight, TOF, and the beamline momentum, $p_{\text {Beam }}$, to reconstruct the invariant mass of the particles in the beamline, $m_{\text {Beam }}$, as follows

$$
m_{\text {Beam }}=\frac{p_{\text {Beam }}}{c} \sqrt{\left(\frac{T O F * c}{l}\right)^{2}-1}
$$

where $c$ is the speed of light and $l$ is the length of the particle's trajectory between the time of flight paddles.

Figure 4.1 shows the mass distribution for the Run II negative polarity runs on the left and positive polarity runs on the right. We perform the classification of events into the different samples as follows:

- $\pi / \mu / e:$ mass $<350 \mathrm{MeV} / \mathrm{c}^{2}$

- kaon: $350 \mathrm{MeV}<\operatorname{mass}<650 \mathrm{MeV} / \mathrm{c}^{2}$

- proton: $650 \mathrm{MeV}<$ mass $<3000 \mathrm{MeV} / \mathrm{c}^{2}$.

Lines 3 and 4 in in Table 4.1 show the number of negative $\pi / \mu / e$ and positive $K$ candidates which pass the mass selection for LArIAT Run-II data.

\subsubsection{TPC Selection: Halo Mitigation}

The secondary beam impinging on LArIAT secondary target produces a plethora of particles which propagates downstream. The presence of upstream and downstream collimators greatly abates the number of particles traveling down the LArIAT tertiary beamline. However, it is possible that more than one particle sneaks into the LArTPC during its readout time: the TPC readout is triggered by the particle firing the series 
Run-II Negative Mass

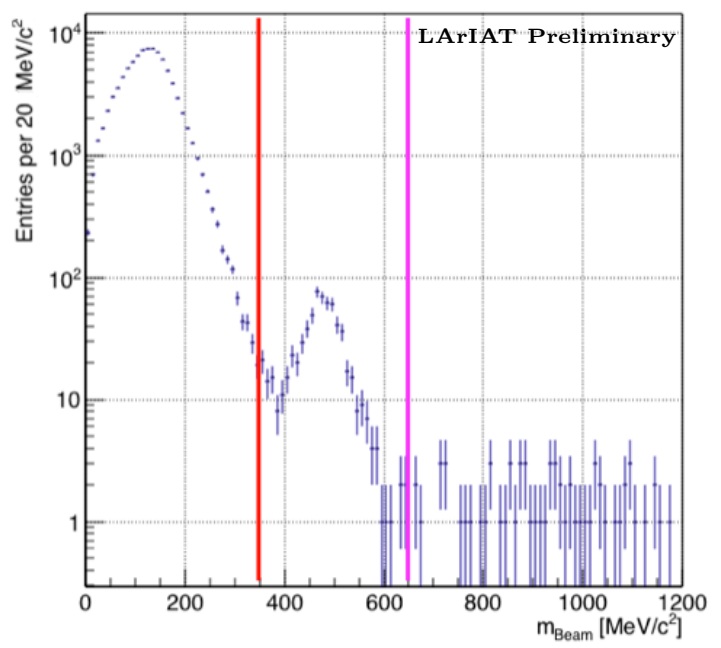

Run-II Positive Mass

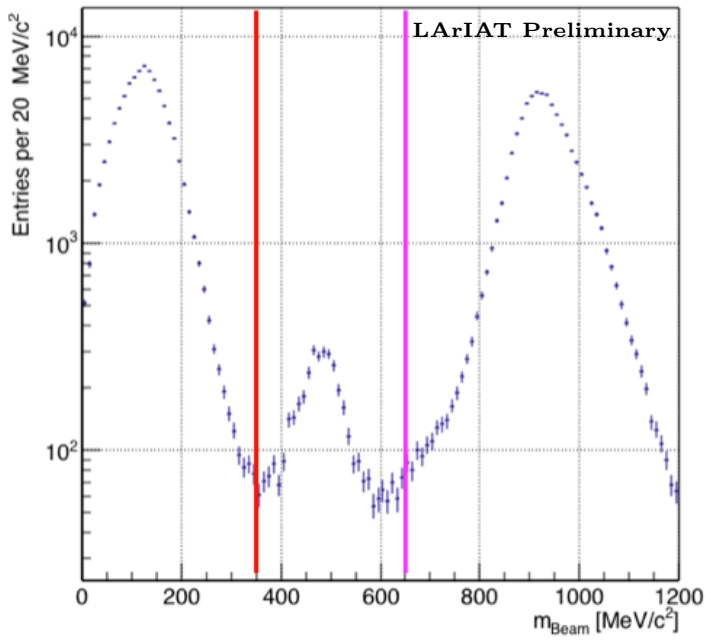

Figure 4.1: Distribution of the beamline mass as calculated according to equation 4.1 for the Run-II events reconstructed in the beamline, negative polarity runs on the left and positive polarity runs on the right. The classification of the events into $\pi^{ \pm} / \mu^{ \pm} / e^{ \pm}, \mathrm{K}^{ \pm}$, or (anti)proton is based on these distributions, whose selection cut are represented by the vertical colored lines.

of beamline detectors along our tertiary beamline, but particles from the beam halo might also be present in the TPC at the same time. We call "pile up" the additional traces in the TPC. We adjusted the primary beam intensity between LArIAT Run I and Run II to reduce the presence of events with high pile up particles in the data sample. For the cross section analyses, we remove events with more than 4 tracks in the first $14 \mathrm{~cm}$ upstream portion of the TPC from the sample (line 5 in in Table 4.1).

\subsubsection{TPC Selection: Shower Removal}

In the case of the $\left(\pi^{-}, \mathrm{Ar}\right)$ cross section, the resolution of beamline mass spectrometer is not sufficient to select a beam of pure pions. In fact, muons which are close in mass to the pions and relativistic electrons survive the selection on the beamline mass. It is important to notice that the composition of the negative polarity beam is mostly pions, as will be discussed in section 5.2.1. Still, we devise a selection on 

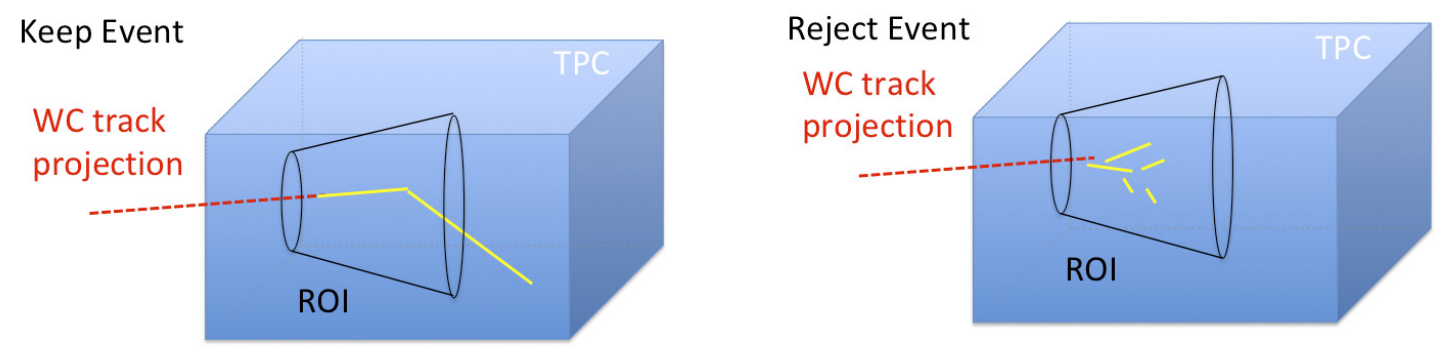

Figure 4.2: Visual rendering of the shower filter. The ROI is a cut cone, with a small radius of $4 \mathrm{~cm}$, a big radius of $10 \mathrm{~cm}$ and an height of $42 \mathrm{~cm}$ (corresponding to 3 radiation lengths for electrons in Argon).

the TPC information to mitigate the presence of electrons in the sample used for the pion cross section. The selection relies on the different topologies of a pion and an electron event when propagating in liquid argon: while the former will trace a track inside the TPC active volume, the latter will tend to "shower", i.e. interact with the medium, producing bremsstrahlung photons which pair convert into several short tracks. In order to remove the shower topology, we create a region of interest (ROI) around the TPC track corresponding to the beamline particle. We look for short tracks contained in the ROI, as depicted in Figure 4.2 if more then 5 tracks shorter than $10 \mathrm{~cm}$ are in the ROI, we reject the event. Line 7 in Table 4.1 shows the number of events surviving this selection; that table also shows that this selection is applied after the beamline event is matched to TPC particle (discussed in the next section). This match already lowers the presence of electrons in the sample, which is further reduced by the shower filter.

\subsection{Beamline and TPC Handshake: the Wire Cham- ber to TPC Match}

For each event passing the selection on its beamline information, we need to identify the track inside the TPC corresponding to the particle which triggered the beamline 

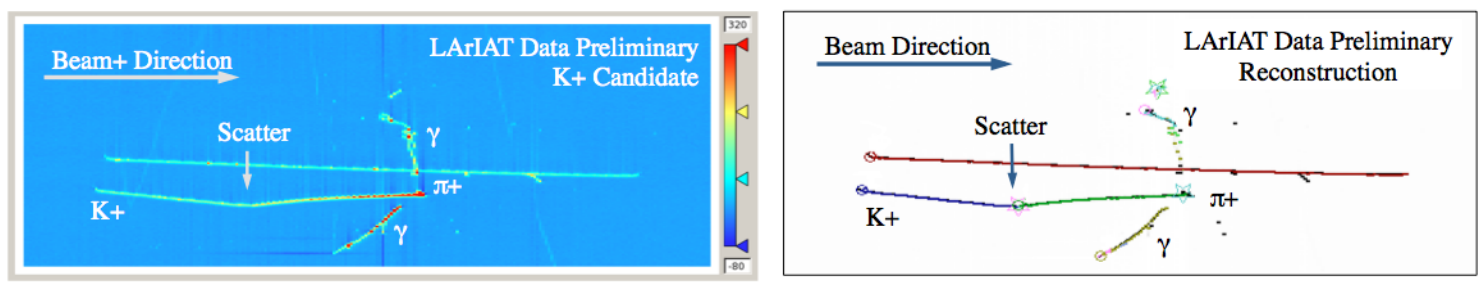

Figure 4.3: Kaon candidate event: on the right, event display showing raw quantities; on the left, event display showing reconstructed tracks. In the reconstructed event display, different colors represent different track objects. A kink is visible in the kaon ionization, signature of a hadronic interaction: the tracking correctly stops at the kink position and two tracks are formed. An additional pile-up track is so present in the event (top track in red).

detectors, a procedure we refer to as "WC to TPC match" (WC2TPC for short). In general, the TPC tracking algorithm can reconstruct more than one track in the event, partially due to the fact that hadrons interact in the chamber and partially because of pile up particles during the triggered TPC readout time, as shown in Figure 4.3 .

We attempt to uniquely match one wire chamber track (see Section 3.2.2) to one and only one reconstructed TPC track. In order to determine if a match is present, we apply a geometrical selection on the relative position of the wire chamber and TPC tracks. We start by considering only TPC tracks whose first point is in the first $2 \mathrm{~cm}$ upstream portion of the TPC for the match. We project the wire chamber track to the TPC front face where we define the coordinates of the projected point as $x_{F F}$ and $y_{F F}$. For each considered TPC track, we define $\Delta \mathrm{X}$ as the difference between the $x$ position of the most upstream point of the TPC track and $x_{F F} . \Delta Y$ is defined analogously. We define the radius difference, $\Delta R$, as $\Delta R=\sqrt{\Delta X^{2}+\Delta Y^{2}}$, see Figure 4.4. We define as $\alpha$ the angle between the incident WC track and the TPC track in the plane that contains them. If $\Delta \mathrm{R}<4 \mathrm{~cm}, \alpha<8^{\circ}$, a match between WC-track and TPC track is found. We describe how we determine the value for the radius and angular selection in Appendix B. We discard events with multiple WC2TPC matches. We use only those TPC tracks that are matched to WC tracks in the cross section calculation. Line 6 in Table 4.1 shows the number of events where a unique WC2TPC 

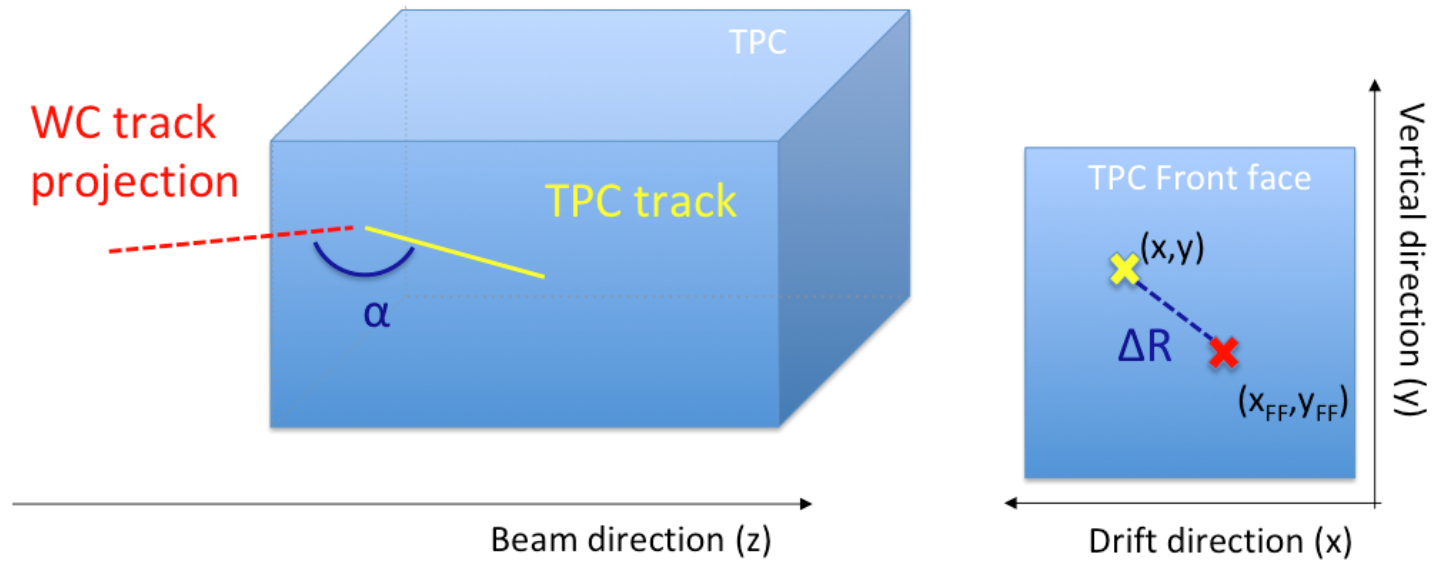

Figure 4.4: Visual rendering of the wire chamber to TPC match.

match was found.

In $\mathrm{MC}$, we mimic the matching between the WC and the TPC track by constructing an artificial WC track using truth information at wire chamber four. We then apply the same WC to TPC matching algorithm as in data.

\subsection{The Thin Slice Method}

Once we have selected the 40841 beamline pion candidates and the 1081 beamline kaon candidates, and we have identified the TPC corresponding track, we apply the thin slice method to measure the cross section, as the following sections describe.

\subsubsection{Cross Sections on Thin Target}

Cross section measurements on a thin target have been the bread and butter of nuclear and particle experimentalists since the Geiger-Marsden experiments [51]. At their core, this type of experiments consists in shooting a beam of particles with a known flux on a thin slab of material and recording the outgoing flux.

In general even in the case of thin target, the target is not a single particle, but rather a slab of material containing many diffusion centers. The so-called "thin 
target" approximation assumes that the target centers are uniformly distributed in the material and that the target is thin compared to the projectile interaction length, so that no center of interaction sits in front of another. In this approximation, the ratio between the number of particles interacting in the target $N_{\text {Int }}$ and the number of incident particles $N_{\text {Inc }}$ on the target estimates the interaction probability $P_{\text {Interacting }}$, which is the complementary to one of the survival probability $P_{\text {Survival }}$. Equation 4.2

$$
P_{\text {Survival }}=1-P_{\text {Interacting }}=1-\frac{N_{\text {Int }}}{N_{\text {Inc }}}=e^{-\sigma_{\text {TOT }} n \delta X}
$$

describes the probability for a particle to survive the thin target. This formula relates the interaction probability to the total hadronic cross section $\left(\sigma_{T O T}\right)$, the density of the target centers $(n)^{1}$ and the thickness of the target along the incident hadron direction $(\delta X)$. If the target is thin compared to the interaction length of the process considered, we can Taylor expand the exponential function in equation 4.2 and find a simple proportionality relationship between the cross section and the number of incident and interacting particles, as shown in equation 4.3 .

$$
1-\frac{N_{\text {Int }}}{N_{\text {Inc }}}=1-\sigma_{T O T} n \delta X+O\left(\delta X^{2}\right)
$$

Solving for the cross section, we find:

$$
\sigma_{T O T}=\frac{1}{n \delta X} \frac{N_{\text {Int }}}{N_{\text {Inc }}}
$$

\subsubsection{Not-so-Thin Target: Slicing the Liquid Argon Volume}

The interaction length of pions and kaons in liquid argon is expected to be of the order of $50 \mathrm{~cm}$ for pions and $100 \mathrm{~cm}$ for kaons. Thus, the LArIAT TPC, with its 90

1. The scattering center density in the target, $n$, relates to the argon density $\rho$, the Avogadro number $N_{A}$ and the argon molar mass $m_{A}$ as $n=\frac{\rho N_{A}}{m_{A}}$. 


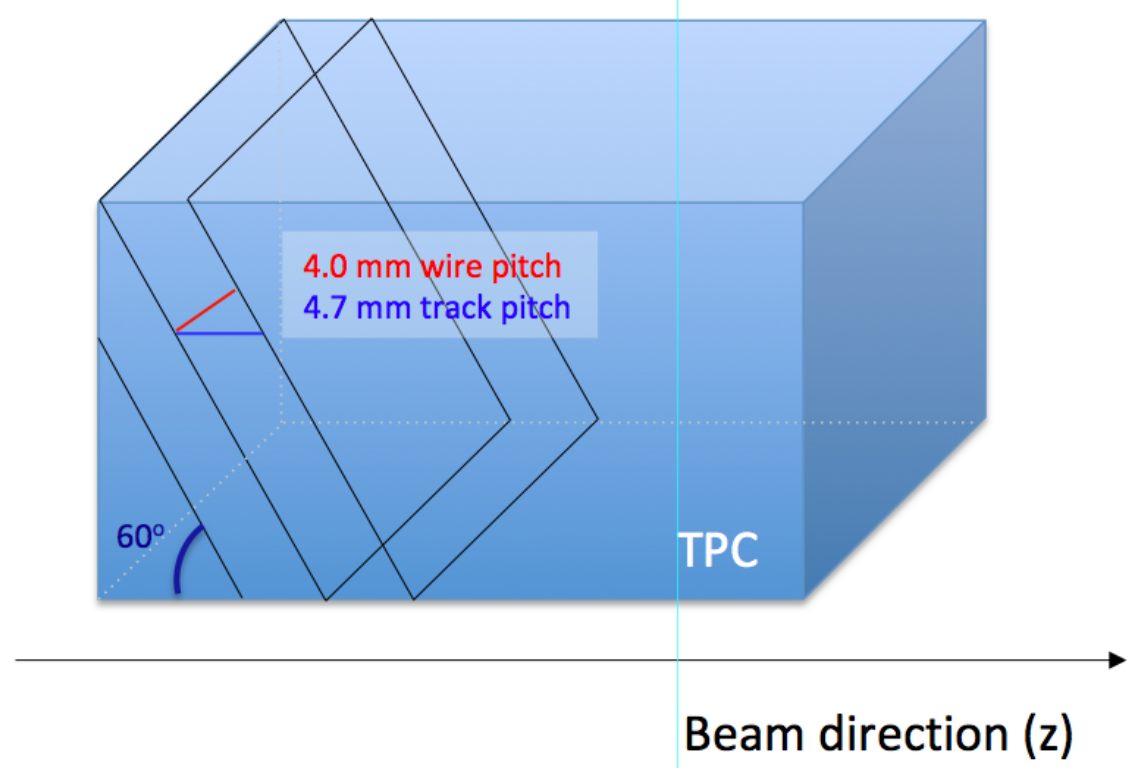

Figure 4.5: Representation of sliced LAr Volume.

$\mathrm{cm}$ of length, is not a thin target. However, the granularity of the LArIAT LArTPC detector allows us to treat the argon volume as a sequence of many adjacent thin targets.

As described in Chapter 3, LArIAT induction and collection planes consist of 240 wires each at $4 \mathrm{~mm}$ spacing. The wires are oriented at $+/-60^{\circ}$ from the vertical direction, while the beam direction is oriented 3 degrees off the $z$ axis in the $X Z$ plane. The collection wires collect signals proportional to the energy deposited by the hadron along its path in a $\delta X=4 \mathrm{~mm} /\left(\sin \left(60^{\circ}\right) \cos \left(3^{\circ}\right)\right) \approx 4.7 \mathrm{~mm}$ slab of liquid argon. Thus, one can think to slice the TPC into many thin targets of $\delta X=4.7 \mathrm{~mm}$ thickness along the direction of the incident particle, making a measurement at each wire along the path, as sketched in Figure 4.5.

Considering each slice $j$ a "thin target", we can apply the cross section calculation from Equation 4.4 iteratively, evaluating the kinetic energy of the hadron as it enters each slice, $E_{j}^{k i n}$. For each WC2TPC matched particle, the energy of the hadron entering the TPC is known thanks to the momentum and mass determination by the tertiary beamline, 


$$
E_{\text {FrontFace }}^{k i n}=\sqrt{p_{\text {Beam }}^{2}-m_{\text {Beam }}^{2}}-m_{\text {Beam }}-E_{\text {loss }},
$$

where $E_{\text {loss }}$ is a correction for the kinetic energy loss in the uninstrumented material between the beamline and the TPC front face. While propagating through the target, the kinetic energy of the hadron at each slab is determined by subtracting the energy deposited by the particle in the previous slabs. For example, at the $j^{\text {th }}$ slab of a track, the kinetic energy will be

$$
E_{j}^{k i n}=E_{\text {FrontFace }}^{k i n}-\sum_{i<j} E_{\text {Dep }, i},
$$

where $E_{\mathrm{Dep}, i}$ is the energy deposited at each argon slice before the $j^{\text {th }}$ point as measured by the calorimetry associated with the tracking.

If the particle enters a slice, it contributes to the $N_{\mathrm{Inc}}\left(E^{k i n}\right)$ distribution in the energy bin corresponding to its kinetic energy in that slice. Within the slice, the hadron may or may not interact. If it interacts in the slice, it contributes also to the $N_{\text {Int }}\left(E^{k i n}\right)$ distribution in the appropriate energy bin; this occurrence corresponds to the end of the hadron tracking. If the hadron does not interact, it will enter the next slice and the interaction evaluation starts again. The process is applied to all the hadrons in the sample; the cross section as a function of kinetic energy, $\sigma_{T O T}\left(E^{k i n}\right)$ is then evaluated to be proportional to the ratio $\frac{N_{\operatorname{Int}}\left(E^{k i n}\right)}{N_{\operatorname{Inc}}\left(E^{k i n}\right)}-$ bin by bin ratio.

Our goal is to measure the total interaction cross section, independently from the topology of the interaction. Thus, we determine that a hadron interacted simply by requiring that the last point of the $\mathrm{WC} 2 \mathrm{TPC}$ matched track lies in a slice within the fiducial volume, whose boundaries are defined in Table 4.2. If the TPC track ends within the fiducial volume, its last point will be the interaction point; if the track crosses the boundaries of the fiducial volume, the track will be considered "through going" and no interaction point will be found. The only points of the hadronic 


\begin{tabular}{|l|r|r|}
\hline & $\min$ & $\max$ \\
\hline$X$ & $1 \mathrm{~cm}$ & $46 \mathrm{~cm}$ \\
\hline$Y$ & $-15 \mathrm{~cm}$ & $15 \mathrm{~cm}$ \\
\hline$Z$ & $0 \mathrm{~cm}$ & $86 \mathrm{~cm}$ \\
\hline
\end{tabular}

Table 4.2: Fiducial volume boundaries used to determine cross section interaction point.

candidate track considered to fill the $N_{\text {Int }}$ and $N_{\text {Inc }}$ distributions are the ones contained in the fiducial volume.

A notable background pertinent only to the $N_{\text {Int }}$ distribution are cases in which the hadrons decays inside the TPC. In those cases in fact, the tracking ends inside the TPC but the interaction is not hadronic. The handling of decay background is treated in a slightly different way for the pion and kaon section, details can be found in sections 5.3 and 7.1 respectively.

\subsubsection{Corrections to the Raw Cross Section}

Equation 4.4 is a prescription for measuring the cross section in case of a pure beam of the hadron of interest and $100 \%$ efficiency in the determination of the interaction point. For example, if LArIAT had a beam of pure pions and were $100 \%$ efficient in determining the interaction point within the TPC, the pion cross section as a function of kinetic energy (estimated at the central value of the energy bin $E_{i}$ ) would be given by

$$
\sigma_{T O T}^{\pi^{-}}\left(E_{i}\right)=\frac{1}{n \delta X} \frac{N_{\mathrm{Int}}^{\pi^{-}}\left(E_{i}\right)}{N_{\mathrm{Inc}}^{\pi^{-}}\left(E_{i}\right)}
$$

Unfortunately, this is not the case. In fact, the selection used to isolate pions in the LArIAT beam allows for the presence of some muons and electrons as background, while the kaon selection allows for a small contamination of protons (see Section 5.2.1). Also, the LArTPC tracking algorithm is not $100 \%$ efficient in deter- 
mining the interaction point. This inefficiency occurs in two fashions: i) the tracking algorithm does not stop at the interaction point and continues adding hits from a particle past it (this happens especially in the case of shallow elastic scattering), ii) the tracking stops prematurely. These two cases have different consequences on the population of the interacting and incident distributions. In the first case, the interacting histogram will be underpopulated and the incident histogram might be overpopulated. In case of premature end of tracking, the interacting histogram will be overpopulated at energies greater than the eventual interaction, while the incident histogram will be underpopulated. Given the importance of tracking for the cross section measurements, we report an optimization to maximize the identification of the interaction point in Appendix B.

Therefore, we apply two corrections evaluated on MC in order to extract the final cross section from LArIAT data: i) a background subtraction and ii) a correction for reconstruction effects. Still using the pion case as example, we estimate the pion cross section in each energy bin changing Equation 4.7 into

$$
\sigma_{T O T}^{\pi^{-}}\left(E_{i}\right)=\frac{1}{n \delta X} \frac{N_{\mathrm{Int}}^{\pi^{-}}\left(E_{i}\right)}{N_{\mathrm{Inc}}^{\pi^{-}}\left(E_{i}\right)}=\frac{1}{n \delta X} \frac{\epsilon^{\mathrm{Inc}}\left(E_{i}\right)\left[N_{\mathrm{Int}}^{\mathrm{TOT}}\left(E_{i}\right)-B_{\mathrm{Int}}\left(E_{i}\right)\right]}{\epsilon^{\mathrm{Int}}\left(E_{i}\right)\left[N_{\mathrm{Inc}}^{\mathrm{TOT}}\left(E_{i}\right)-B_{\mathrm{Inc}}\left(E_{i}\right)\right]}
$$

where $N_{\text {Int }}^{\mathrm{TOT}}\left(E_{i}\right)$ and $N_{\text {Incident }}^{\mathrm{TOT}}\left(E_{i}\right)$ is the measured content of the interacting and incident histograms for events that pass the event selection, $B_{\text {Int }}\left(E_{i}\right)$ and $B_{\text {Inc }}\left(E_{i}\right)$ represent the contributions from the background to the interacting and incident histograms respectively, and $\epsilon^{\mathrm{Int}}\left(E_{i}\right)$ and $\epsilon^{\mathrm{Inc}}\left(E_{i}\right)$ are the corrections for reconstruction effects.

As we will show in Section 5.3 , the background subtraction for the interacting and incident histograms can be translated into corresponding relative pion content factors $C_{\mathrm{Int}}^{\pi M C}\left(E_{i}\right)$ and $C_{\mathrm{Inc}}^{\pi M C}\left(E_{i}\right)$ and the cross section re-written as follows 


$$
\sigma_{T O T}^{\pi^{-}}\left(E_{i}\right)=\frac{1}{n \delta X} \frac{\epsilon^{\mathrm{Inc}}\left(E_{i}\right) C_{\mathrm{Int}}^{\pi M C}\left(E_{i}\right) N_{\mathrm{Int}}^{\mathrm{TOT}}\left(E_{i}\right)}{\epsilon^{\mathrm{Int}}\left(E_{i}\right) C_{\mathrm{Inc}}^{\pi M C}\left(E_{i}\right) N_{\mathrm{Inc}}^{\mathrm{TOT}}\left(E_{i}\right)} .
$$

\subsection{Procedure testing with MC truth quantities}

The $\left(\pi^{-}, \mathrm{Ar}\right)$ and $\left(\mathrm{K}^{+}, \mathrm{Ar}\right)$ total hadronic cross section implemented in Geant4 can be used as a tool to validate the measurement methodology. We describe here a closure test done on Monte Carlo to prove that the methodology of slicing the TPC retrieves the underlying cross section distribution implemented in Geant4 within the MC statistical uncertainty.

For pions and kaons in the considered energy range, the Geant4 inelastic model adopted is "BertiniCascade"; the pion elastic cross sections are tabulated from Chips, while the kaon elastic cross sections are tabulated on Gheisha and Chips.

For the validation test, we fire a sample of pions and a sample of kaons inside the LArIAT TPC active volume using the Data Driven Monte Carlo, a procedure described in Section 5.2.2. We apply the thin-sliced method using only true quantities to calculate the hadron kinetic energy at each slab in order to decouple reconstruction effects from possible issues with the methodology. For each slab of $4.7 \mathrm{~mm}$ length along the path of the hadron, we integrate the true energy deposition as given by the Geant4 transport model. Then, we recursively subtracted it from the hadron kinetic energy at the TPC front face to evaluate the kinetic energy at each slab until the true interaction point is reached. Since the MC is a pure beam of the hadron of interest and truth information is used to retrieve the interaction point, no background correction or reconstruction effects correction is applied. Doing so, we obtain the true interacting and incident distributions for the considered hadron, whose ratio leads to the true MC cross section as a function of the hadron kinetic energy.

Figure 4.6 shows the total hadronic cross section for argon implemented in Geant4 
Cross Sections for pi- off $\mathrm{Ar}$

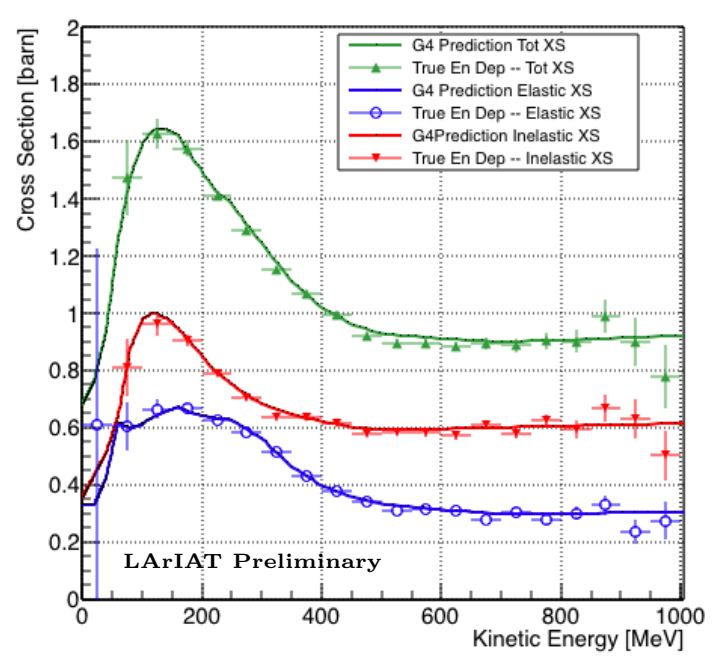

$\mathrm{K}^{+} \mathrm{Ar}$ Cross Section

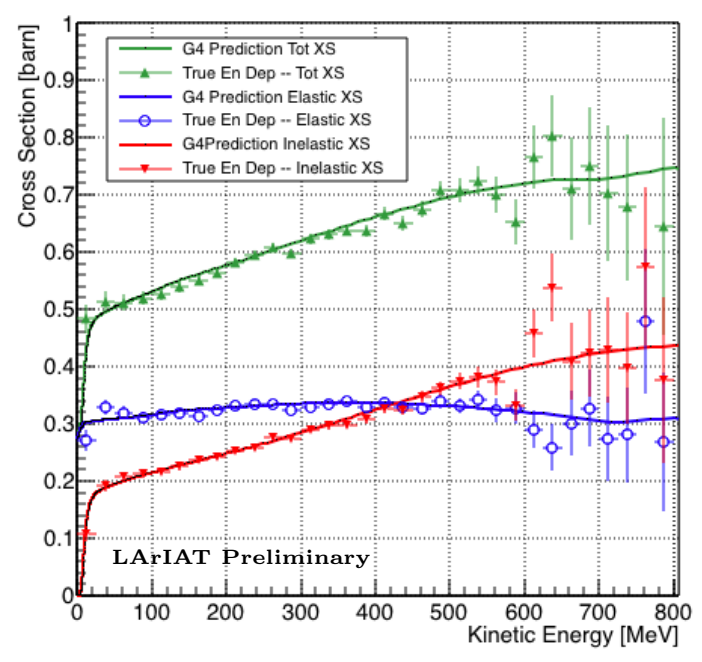

Figure 4.6: Hadronic cross sections for $\left(\pi^{-}, \mathrm{Ar}\right)$ on the left and $\left(\mathrm{K}^{+}, \mathrm{Ar}\right)$ on the right as implemented in Geant4 10.03.p1 (solid lines) overlaid the true MC cross section as obtained with the sliced TPC method (markers). The total cross section is shown in green, the elastic cross section in blue and the inelastic cross section in red.

10.03.p1 (solid lines) overlaid with the true MC cross section as obtained with the sliced TPC method (markers) for pions on the left and kaons on the right; the total cross section is shown in green. For completeness, we also report the contributions from the elastic cross section (in blue) and the inelastic cross section (in red), available at the MC level. The nice agreement with the Geant4 distribution and the cross section obtained with the sliced TPC method gives us confidence in the validity of the methodology. 


\section{Chapter 5}

\section{Data and MC preparation for the Cross Section Measurements}

"Il dolce non lo mangi mai, ma qualche volta ti rifai. Abbracciami" - Pietro Ciampi, 1971 -

This chapter describes the work done on the data and Monte Carlo samples in preparation for the cross section analyses. This entails the choice of the datasets and the production of the information needed to construct the Monte Carlo Simulation (Section 5.1), the construction and use of said Monte Carlo simulation (Section 5.2 , the study of backgrounds for the pion cross section (Section 5.3), the study of the energy loss between WC4 and TPC (Section 5.4), the study of the tracking in the TPC (Section 5.5), and study of the calorimetry response (Section 5.6).

\subsection{Cross Section Analyses Data Sets}

We choose LArIAT Run-II as the data period for the $\left(\pi^{-}, \mathrm{Ar}\right)$ and $\left(\mathrm{K}^{+}, \mathrm{Ar}\right)$ total hadronic cross section analyses. Data taking for the this period started on 03/15/2016 
and ended on $07 / 31 / 2016$. Since we are interested in beamline and TPC information, we ask basic requirements on the operational status of the time of flight counters, wire chambers and TPC to form the good run list for this period, which we informally call "lovely runs".

The subset of lovely runs chosen for the $\left(\pi^{-}, \mathrm{Ar}\right)$ total hadronic cross section analysis includes only the $-60 \mathrm{~A}$ and $-100 \mathrm{~A}$ magnet configurations in negative polarity, even if LArIAT explored several other beamline configurations during Run-II. The $60 \mathrm{~A}$ and -100A combined data set accounts for approximately $90 \%$ of the total Run-II negative polarity runs. The choice of these two main beamline settings limits the need for the production of many different MC sets and related corrections, still maintaining a high number of events.

Similarly, the subset of lovely runs chosen for the $\left(\mathrm{K}^{+}, \mathrm{Ar}\right)$ total hadronic cross section analysis includes only the $+60 \mathrm{~A}$ and $+100 \mathrm{~A}$ magnet configurations in positive polarity. It should be noted that kaons are extremely rare in the $+60 \mathrm{~A}$ sample, thus the data sample for the $\left(\mathrm{K}^{+}, \mathrm{Ar}\right)$ cross section after the mass selection is about $90 \%$ +100 A runs, as shown in Table 5.1.

For these first measurements that make use of both the LArIAT beamline and TPC information, we choose strict requirements on the reconstruction of the WC tracks, the so-called "Picky Track" sample (see Section 3.2.2), where we require a single hit in each and every wire chamber detector to reconstruct the WC track. This choice presents two advantages: the uncertainty on the momentum reconstruction for the "Picky Tracks" sample is smaller compared to the "High Yield" sample, and the comparison with the beamline MC results is straightforward. A possible future update and cross check of these analysis would be the use of the High Yield sample, where the statistics is about three times higher.

The breakdown of beamline events as a function of the magnets settings is shown in Table 5.1. The choice of the data sets determines the production of beamline MC 
and serves as basis for the production of Data Driven MC, as shown in the next sections.

\subsection{Construction of a Monte Carlo Simulation for LArIAT}

For the simulation of LArIAT events and for the simulation of the datasets' particle make up, we use a combination of two MC generators: the G4Beamline Monte Carlo and the Data Driven single particle Monte Carlo (DDMC). We use the G4Beamline MC to simulate the particle transport in the LArIAT tertiary beamline and calculate the particle composition of the beam just after the last Wire Chamber (WC4). We use the DDMC to simulate the particles after WC4 along the beamline, close to the beam window in the LAr cryostat and in the TPC.

\subsubsection{G4Beamline}

G4Beamline simulates the beam collision at the LArIAT secondary target, the energy deposited by the particles in the LArIAT beamline detectors, and the action of the LArIAT magnets, effectively accounting for particle transport through the beamline from the LArIAT target until "Big Disk", a fictional, void detector located just before the LArIAT cryostat. At the moment of this writing, G4Beamline does not simulate the responses of the beamline detectors. It is possible to interrogate the truth level information of the simulated particles in several points of the geometry. In order

\begin{tabular}{|l|c|c|c|}
\hline & $\mathrm{I}=60 \mathrm{~A}$ & $\mathrm{I}=100 \mathrm{~A}$ & Total \\
\hline Data Events after $\pi / \mu / e$ Mass Selection & 67068 & 71413 & 138481 \\
\hline Data Events after $K$ Mass Selection & 274 & 2563 & 2837 \\
\hline
\end{tabular}

Table 5.1: Number of data events which fit the $\pi / \mu / e$ or $K$ mass hypothesis as a function of magnet settings. 
to ease the handshake between G4Beamline and the DDMC, we ask for the beam composition just after WC4. Since LArIAT data are taken under different beam conditions, we need to simulate separately the beam composition according to the magnets' settings and the secondary pion beam intensity with G4Beamline. For the pion cross section analysis the relevant beam conditions are secondary pion beam energy of $64 \mathrm{GeV}$, negative polarity magnet with current of $100 \mathrm{~A}$ and $60 \mathrm{~A}$. For the kaon cross section analysis the relevant beam conditions is a secondary pion beam energy of $64 \mathrm{GeV}$, positive polarity magnet with current of $100 \mathrm{~A}$.

\section{Beam Composition for Negative Pion Cross Section}

Even if pions are by far the biggest beam component in negative polarity runs, the LArIAT tertiary beam is not a pure pion beam. While useful to discriminate between pions, kaons, and (anti)protons, the beamline detectors are not sensitive enough to discriminate among the lighter particles in the beam: electrons, muons and pions fall under the same mass hypothesis. Thus, we need to assess the contamination from beamline particles other than pions in the event selections used for the pion cross section analysis and correct for this background. The first step of this process is assessing the percentage of electrons and muons in the $\pi / \mu / e$ beamline candidates via the G4Beamline MC, as we deem the percentage of kaons and antiprotons negligible after the mass selection $(<1 \% 0)$. Since the beamline composition is a function of the magnet settings, we simulate separately events for magnet current of $-60 \mathrm{~A}$ and -100A. Figure 5.1 shows the momentum predictions from G4Beamline overlaid with data for the $60 \mathrm{~A}$ runs (left) and for the 100A runs (right). The predictions for electrons, muons and pions have been staggered and their sum is area normalized to data. Albeit not perfect, these plots show a reasonable agreement in momentum shapes between data and MC. We attribute the difference in shape (longer tail in data) to a two approximations performed in the MC. Firstly, G4Beamline lacks the 
Beamline Composition -- $64 \mathrm{GeV} \pi$-60A Magnet Current

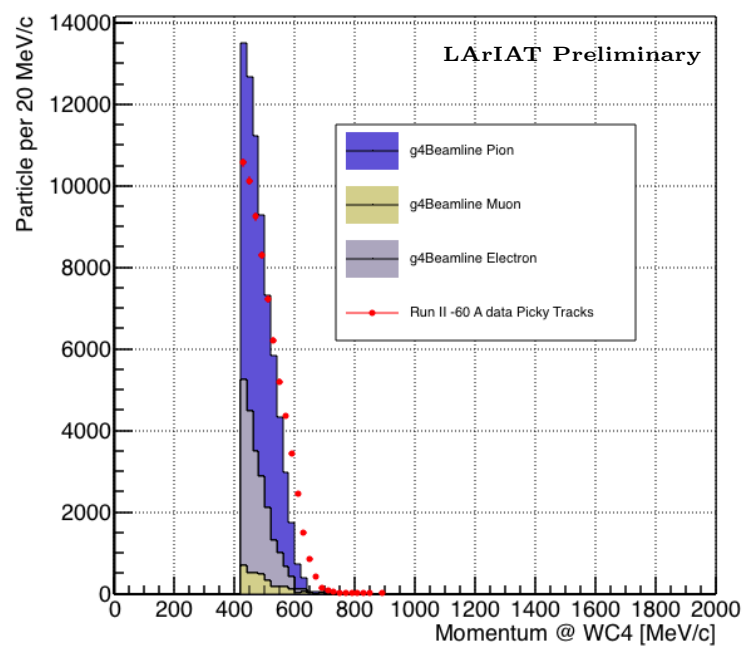

Beamline Composition -- $64 \mathrm{GeV} \pi^{\prime}-100 \mathrm{~A}$ Magnet Current

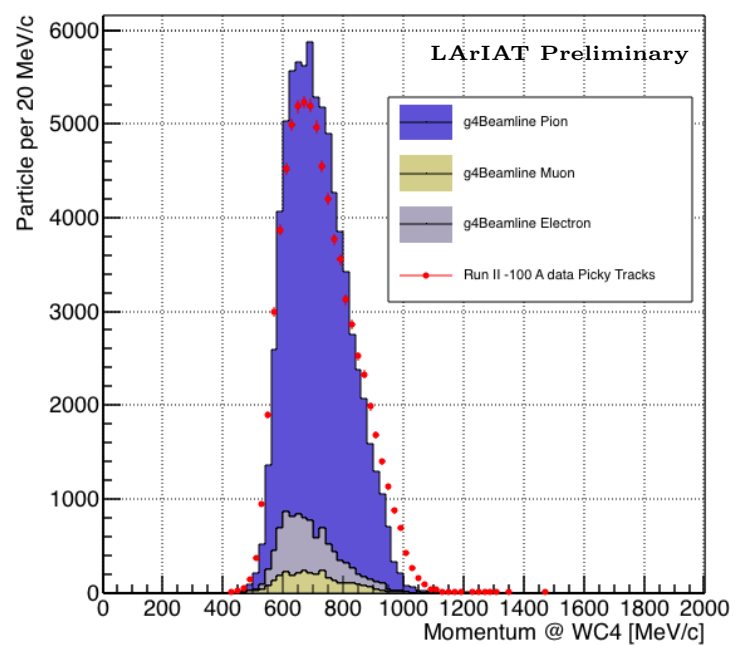

Figure 5.1: Beam composition for the $-60 \mathrm{~A}$ runs (left) and -100A runs (right). The solid blue plot represents the simulated pion content, the yellow plot represents the simulated muon content and the grey plot represents the simulated electron content. The plots are area normalized to the number of data events, shown in red.

\begin{tabular}{|l|c|c|}
\hline & $\mathrm{I}=-60 \mathrm{~A}$ & $\mathrm{I}=-100 \mathrm{~A}$ \\
\hline G4Pions & $68.8 \%$ & $87.4 \%$ \\
\hline G4Muons & $4.6 \%$ & $3.7 \%$ \\
\hline G4Electrons & $26.6 \%$ & $8.9 \%$ \\
\hline
\end{tabular}

Table 5.2: Simulated beamline composition per magnet settings

simulation of the WC efficiency which is momentum dependent and leads to enhance the number events in the center of the momentum distribution. Secondly, G4Beamline stop tracking pions and their products if they decay after $\mathrm{WC1}$; in data, pion decays in flight can still create a tigger if the produced muon travels through the beamline detectors. In the pion cross section analysis, these differences between data and the G4Beamline prediction are accounted for as a systematic uncertainty related to the beam composition (see Section 6.2.1).

Table 5.2 shows the beam composition per magnet setting after the mass selection according to the G4Beamline simulation.

The estimated beam composition is used as a basis to estimate the background contamination in the $\left(\pi^{-}, \mathrm{Ar}\right)$ cross section measurement, whose full treatment is 
described in Section 5.3 .

\section{Beam Composition for Positive Kaon Cross Section}

In the positive polarity runs, the tertiary beam composition is mainly pions and protons. The left side of Figure 5.2 shows the predictions for the momentum spectra for the 100A positive runs according to G4Beamline (solid colors) overlaid with data (black points). Since the LArIAT beamline detectors can discriminate between kaons and other particles, we do not rely on the G4Beamline simulation to estimate the beamline contamination in the pool of kaon candidates (as in the case of the pion cross section), but rather we use a data drive approach. The basic idea of this data driven approach is to estimate the bleed over from high and low mass peaks under the kaon peak by fitting the tails of the $\pi / \mu / e$ and proton mass distributions, as shown in Figure 5.2 right side. Since the shape of the tails is unknown, the estimate is done multiple times varying the range and shape for reasonable functions. For example, to estimate the proton content under the kaon peak, we start by fitting the left tail of the proton mass distribution with a gaussian function between $650 \mathrm{MeV} / \mathrm{c}^{2}$ and $750 \mathrm{MeV} / \mathrm{c}^{2}$. We extend the fit function under the kaon peak and integrate the extended fit function between $350-650 \mathrm{MeV} / \mathrm{c}^{2}$. We integrate the mass histogram in the same range and calculate the proton contamination as the ratio between the two integrals. We repeat this procedure for several fit shapes (gaussian, linear and exponential functions) and tail ranges. Finally, we calculate the contamination as the weighted average of single estimates, where the weights are calculated to be the 1./|1- $\chi^{2} \mid$ of the tail fits. The procedure is repeated for lighter particles mass peak independently. With 12 iterations of this method we find a proton contamination of $5.0 \pm 2.0 \%$ and a contamination from the lighter particles of $0.2 \pm 0.5 \%$. The estimate of the proton background is currently not used in the kaon cross section analysis, but it is a fundamental step to retrieve the true kaon cross section which 

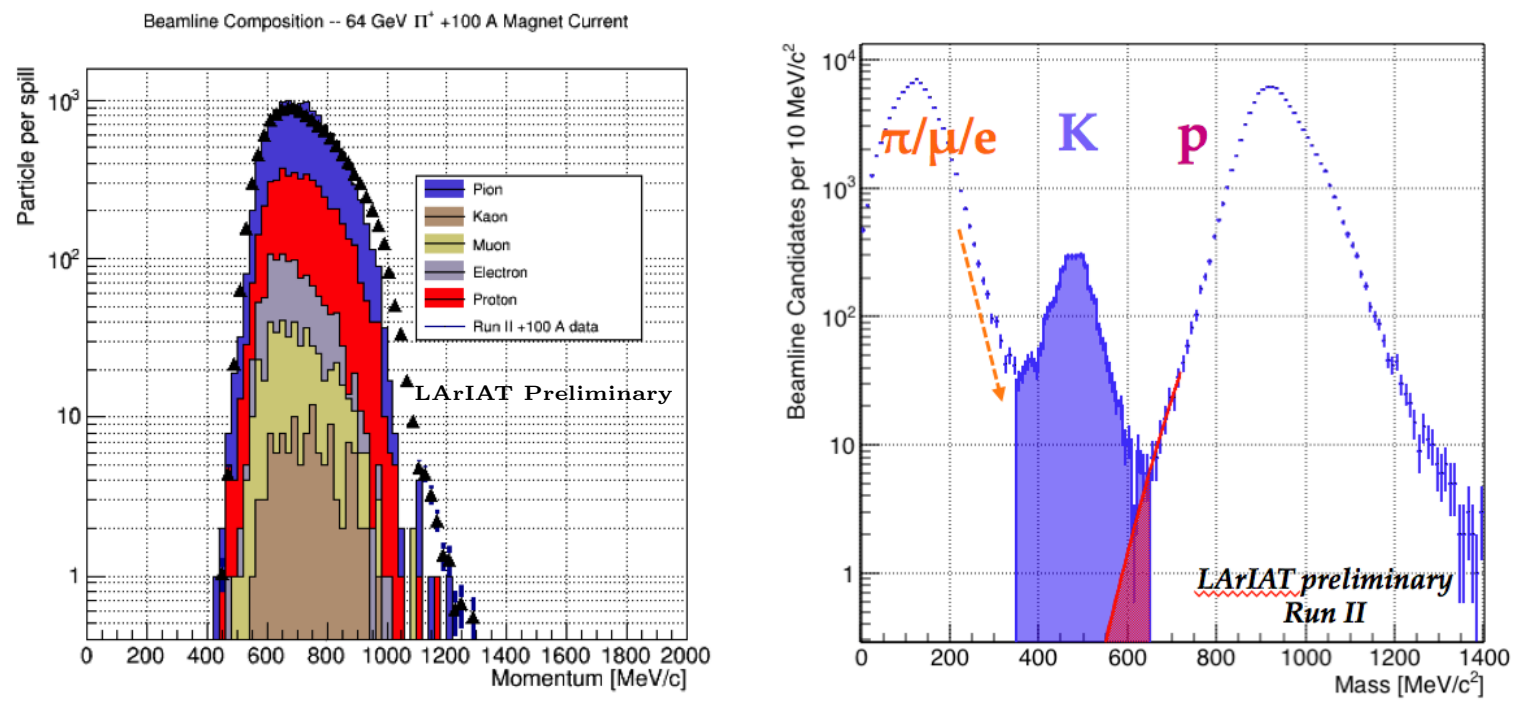

Figure 5.2: Left: Beam composition for the +100A runs after WC4 (no mass selection applied). The solid colors represent the contributions from the G4Beamline simulated particles: blue plot represents the simulated pion content, the yellow plot represents the simulated muon content and the grey plot represents the simulated positron content, the red the proton content and the mustard the kaon content. The plots are area normalized to the number of data events, shown in black. Right: Mass distribution for the Run-II positive runs, where the area under the kaon mass peak is highlighted in purple. The area under the extension of a possible fit for the proton tail is highlighted in red.

will be implemented in the further development of the analysis.

\subsubsection{Data Driven MC}

The Data Driven single particle Monte Carlo (DDMC) is a single particle gun which simulates the particle transport from WC4 into the TPC leveraging on the beamline data information. The DDMC uses the data momentum and position at WC4 to derive the event generation: a general sketch of the DDMC workflow is shown in Figure 5.3 .

When producing a DDMC sample, beamline data from a particular running period and/or running condition are selected first. For example, data for the negative $60 \mathrm{~A}$ runs and for the negative 100A runs inform the event generation stage of two different DDMC samples. Figure 5.4 schematically shows the data quantities of in- 
terest leveraged from data: the momentum $\left(P_{x}, P_{y}, P_{z}\right)$ and position $(X, Y)$ at WC4. For each data event, we obtain the particle position $(X, Y)$ at WC4 directly from the data measurement; we calculate the components of the momentum using the beamline measurement of the momentum magnitude in conjunction with the hits on WC3 and WC4 to determine the direction of the momentum vector, as described in Section 3.2.2. The momentum and position of the selected data events form a 5-dimensional series of tuples. The DDMC event generator samples from the joint distribution of these five quantities using a 5-dimensional hit-or-miss sampling procedure. This sampling generates $\mathrm{MC}$ events with the same momentum and position distributions as data, with the additional benefit of accounting for the correlations between the $P_{x}, P_{y}, P_{z}, X, Y$ variables. As an example, the results of the DDMC generation compared to data for the kaon $+100 \mathrm{~A}$ sample are shown in figure 5.5 for the $P_{z}, X$ and $Y$ distributions; as expected, MC and data agree within the statistical uncertainty by construction. A LArSoft simulation module then launches single particle MC from $\mathrm{z}=-100 \mathrm{~cm}$ (the location of the WC4) using the generated events. The particles are free to decay and interact in their path from WC4 to the TPC according to the Geant4 simulation.

Using the DDMC technique ensures that the MC and data particles have very similar momentum, position and angular distributions at WC4 and allows us to use the MC sample in several occasions: to estimate the background contamination to the pion cross section (see Section 5.3), to calibrate the energy loss upstream of the TPC (see Section 5.4), and to study the tracking and the calorimetric performance in the LArTPC (Sections 5.5 and 5.6). A small caveat is in order here: the DDMC is a single particle Monte Carlo, which means that the beam pile-up is not simulated.

We generate six samples for the pion cross section measurement: three samples of $\sim 330000$ pions, muons and electrons to simulate the negative $60 \mathrm{~A}$ runs, and three samples of $\sim 340000$ pions, muons and electrons for the negative 100A runs. We 
generate a sample of 195000 kaons for the kaon cross section analysis.

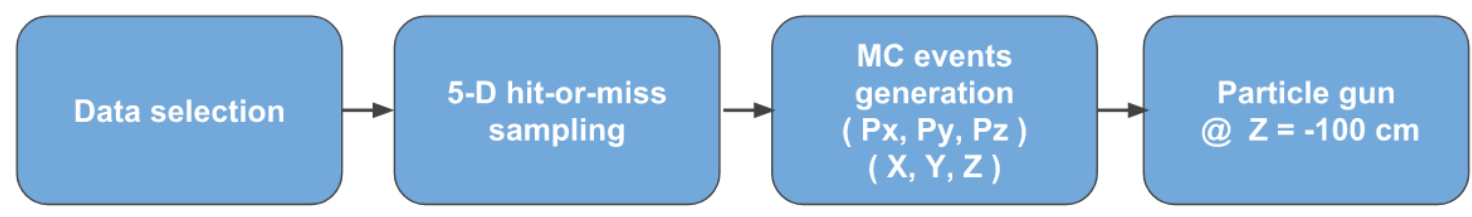

Figure 5.3: Workflow for Data Driven single particle Monte Carlo production.
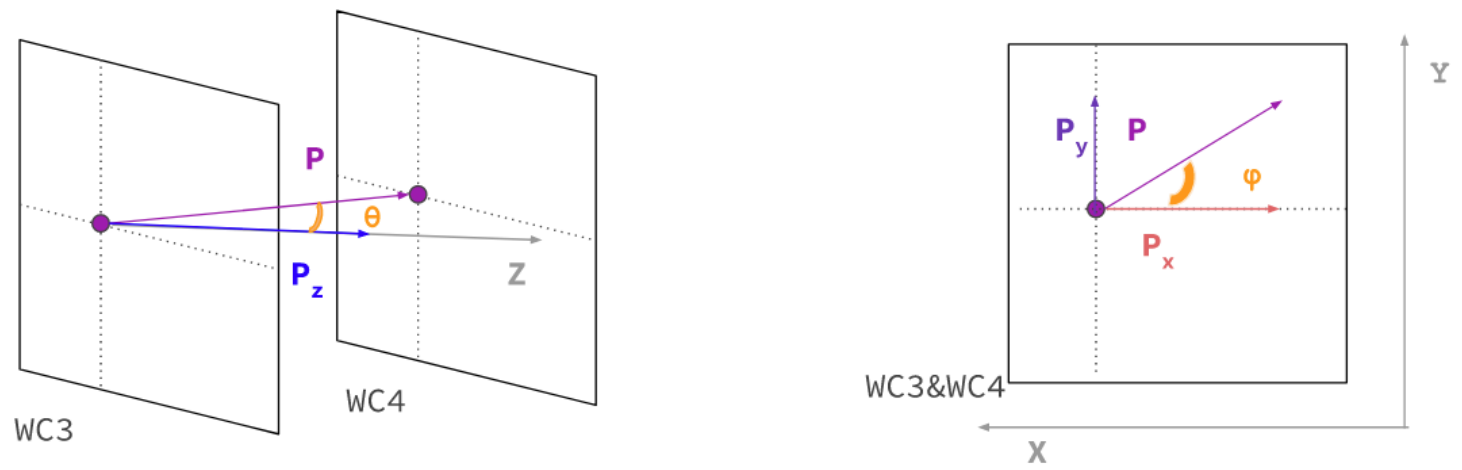

Figure 5.4: Scheme of the quantities of interest for the DDMC event generation: $P_{x}, P_{y}, P_{z}, X, Y$ at WC4.

\subsection{Estimate of Backgrounds in the Pion Cross Section}

We use the beamline simulation and the DDMC simulation to estimate the background in the total hadronic pion cross section. Two categories of background exists for the negative pion cross section measurement: the background related to the pion alternative processes to hadronic interaction inside the LArTPC, discussed in Section 5.3.1 and the background related to the beamline contamination, discussed in Section 5.3 .2 . 

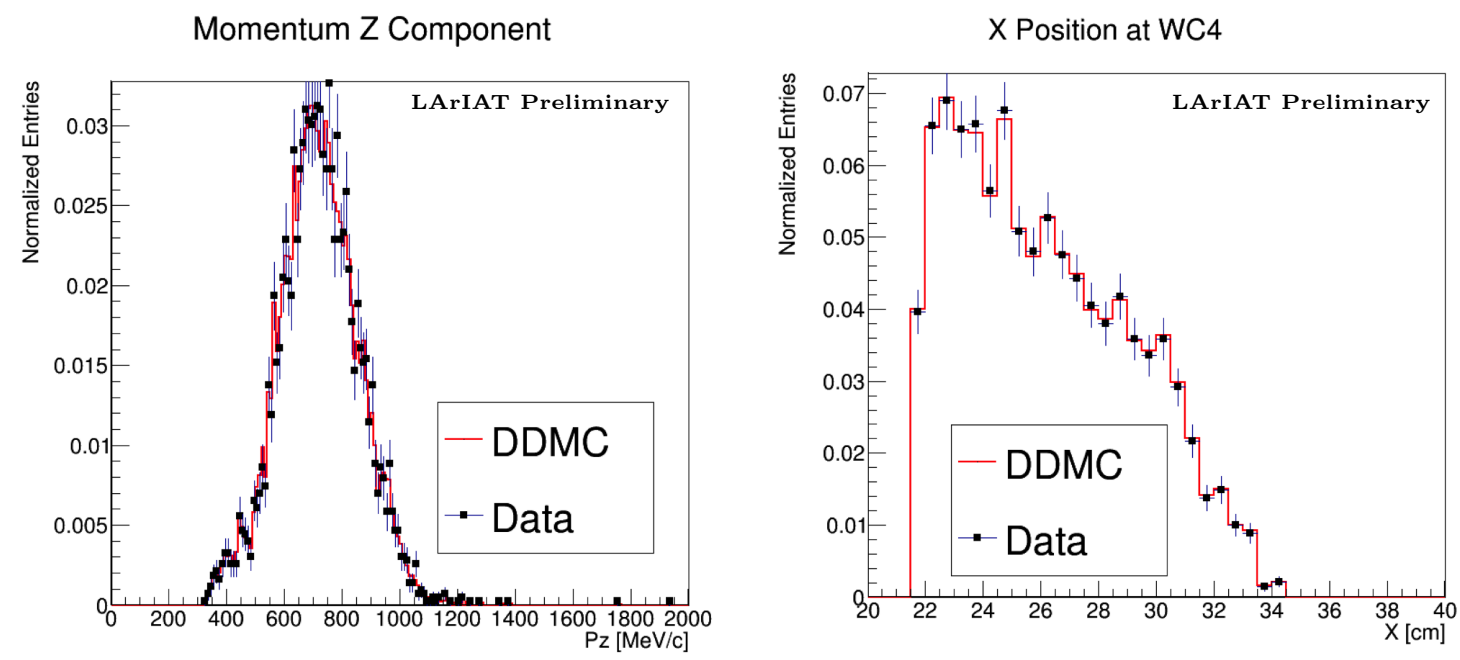

Y Position at WC4

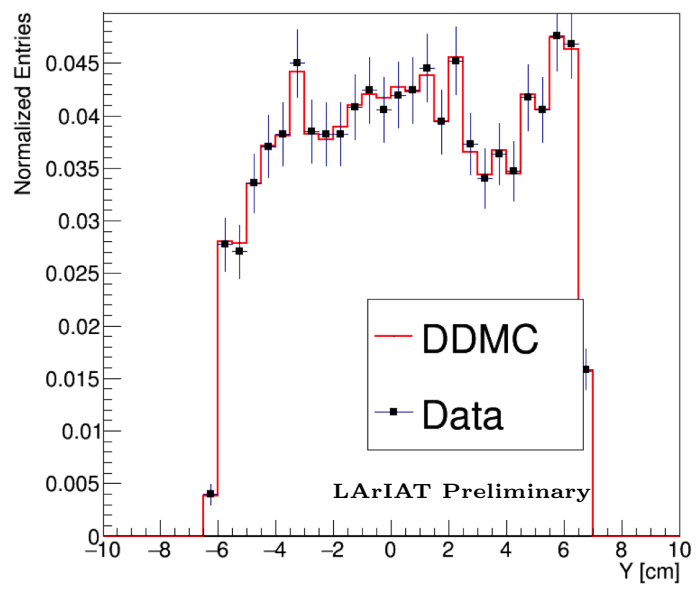

Figure 5.5: Comparison between generated quantities and data distributions for the 100A kaon sample: Z component of the momentum at WC4 (top left), X position at Wire Chamber 4 (top right), Y position at Wire Chamber 4 (bottom). 


\subsubsection{Background from Pion Capture and Decay}

Our goal is to measure the total hadronic cross section for negative pions in argon. Since pion capture can be classified as an electromagnetic process and pion decay is a weak process (as discussed in Section 1.4.1), capture and decay represent alternative processes to the hadronic interaction, resulting therefore into a background for the cross section analysis. We present here a study of capture and decay in Monte Carlo and the solution we adopted to remove their occurrence in the data sample.

For this MC study, we use a sample of MC pions generated according to the $-60 \mathrm{~A}$ beam profile with the DDMC (see Section 5.2.2). It is important to notice that capture occurs predominantly at rest, while decay may occur both in flight and at rest. Thus, we can highly mitigate capture and decay at rest by removing pions whose kinetic energy at the TPC front face is low enough to be completely released by ionization in the TPC, eventually bringing the pion to stop within the fiducial volume. This translates into a beam momentum selection, where we keep only events whose $\mathrm{WC}$ momentum is above a certain threshold. Figure 5.6 shows the true momentum distribution for the primary pions $\mathrm{I}^{1}$ that arrive to the TPC (pink), that capture (green) or decay (blue) inside the TPC, on a linear scale (left) and on a $\log$ scale (right) vertical axis.

In order to choose the selection value for the wire chamber momentum, it is beneficial to estimate the fraction of $\mathrm{MC}$ capture and decay events that survive the momentum selection as a function of the momentum threshold, and compare it with the survival fraction for all the 60A events. This is done in Figure 5.7. We define the survival ratio simply as the number of events surviving the true momentum selection divided by the number of events of that category. We calculate the survival ratio

1. We use here the Geant4 denomination "primary" to indicate that the pion considered does not undergo interactions modifying its energy before getting to the TPC. In fact, not every pion shot from wire chamber four will arrive to the TPC as primary, some will decay or interact before the TPC. 
True Momentum @ WC4 of G4 primaries

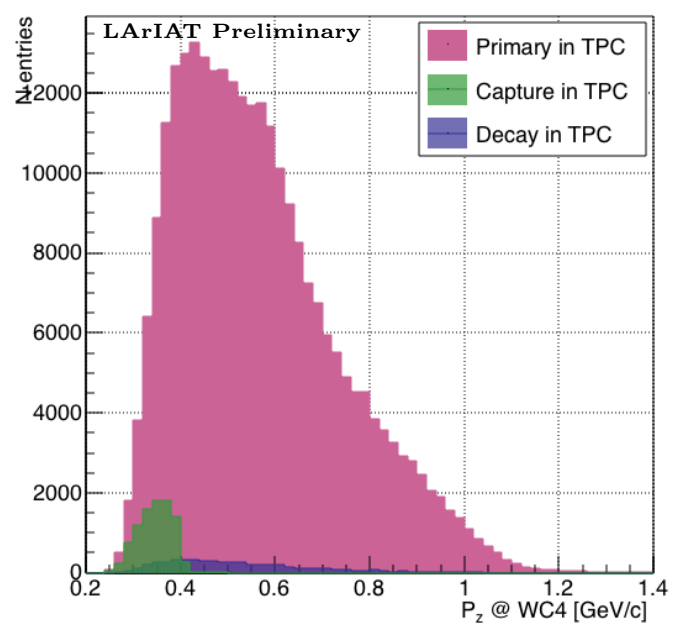

True Momentum @ WC4 of G4 primaries

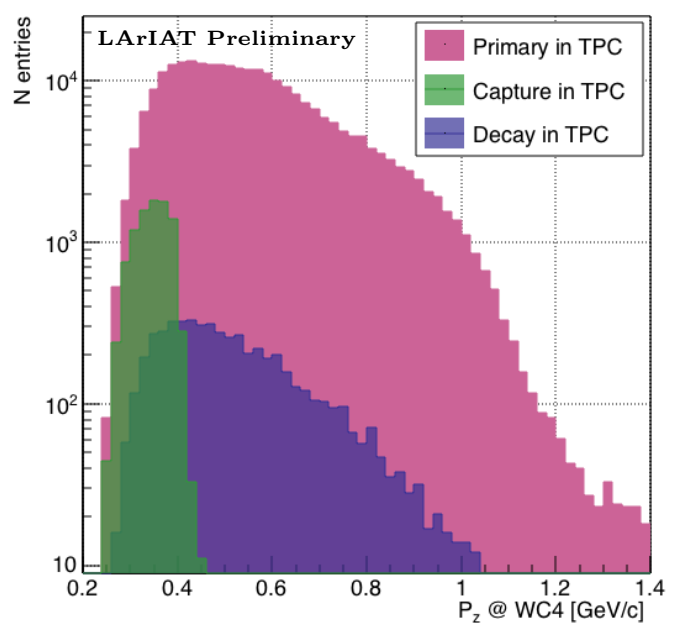

Figure 5.6: MC momentum distribution at wire chamber 4 for every simulated pion arriving in the TPC (pink), ending its life in capture (green) or in decay (blue) in the $\mathrm{TPC}$, linear vertical axis on the left, logarithmic on the right.

separately for the three event categories explained above: total (pink), capture (green) and decay (blue). Selecting pions with momentum greater than $420 \mathrm{MeV} / \mathrm{c}$ removes $\sim 99 \%$ the capture events while maintaining about $80 \%$ of the $60 \mathrm{~A}$ data sample and almost the entire 100A sample. Figure 5.8 shows the ratio of events which end their life in capture (green) or decay (blue) over the total number of events as a as a function of the true MC momentum at WC4. This ratio is slightly dependent on the inelastic cross section implemented in Geant4, as we are able to register a pion capture (or decay) only if it did not interact inelastically in the TPC. We choose a momentum threshold of $420 \mathrm{MeV} / \mathrm{c}$ because the percentage of capture events drops below $1 \%$ and the percentage of decays is never above $2 \%$ for momenta greater than $420 \mathrm{MeV} / \mathrm{c}$. After the momentum selection, we evaluate the contribution of capture and decay to be a negligibly small background to the cross section measurement compared to the background related to the beamline which we will address in the next section. 


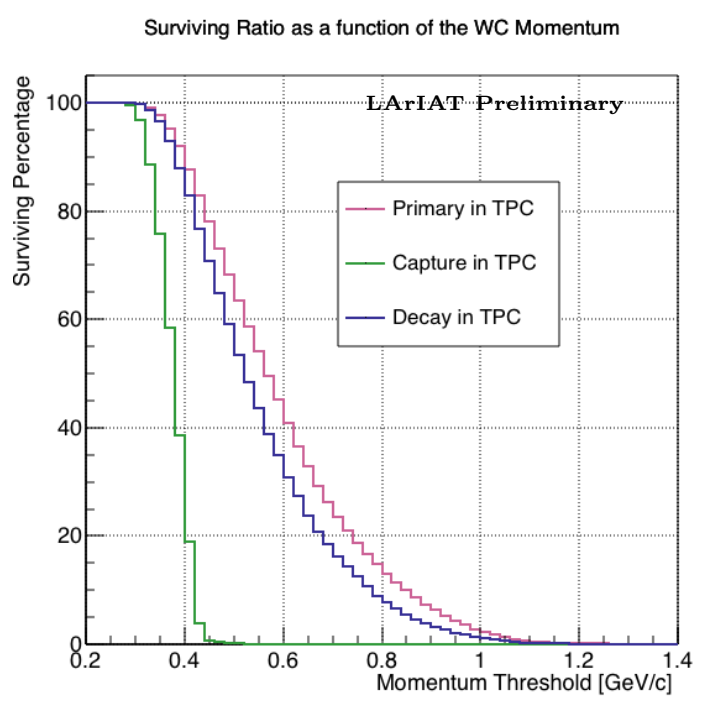

Figure 5.7: Survival ratio as a function of selection threshold on MC momentum at wire chamber four for for every simulated pion arriving in the TPC (pink), capture (green) or in decay (blue).
Event Ratio as function of momentum

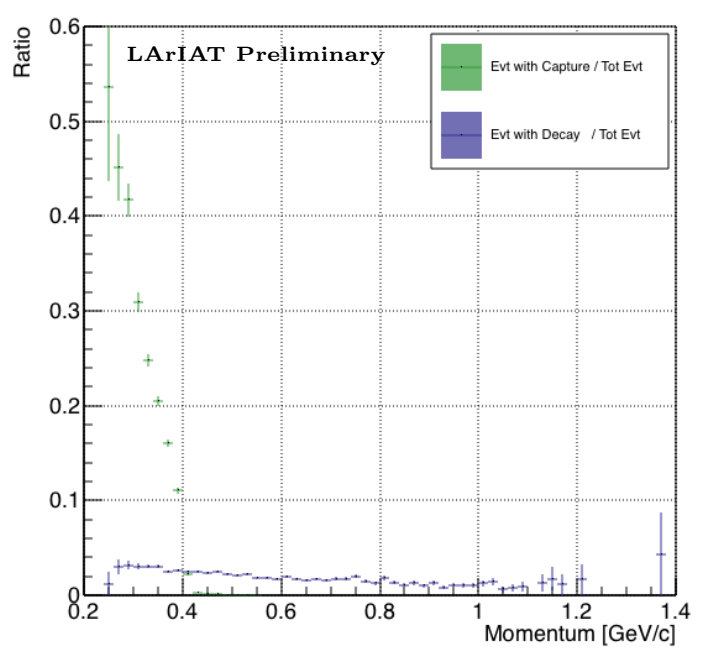

Figure 5.8: Ratio between the capture (green) and decay (blue) events over the total number of events as a as a function of the MC momentum at wire chamber four. 


\subsubsection{Contributions from the Beamline Background}

We define "beamline background" as every TPC track matched to the WC track which is not a primary pion. Potentially, there are 4 different types of beamline background:

1) electrons,

2) muons,

3) secondaries from pion interactions in beamline elements,

4) matched pile up events.

The first step to quantify the effect of the beamline background on the pion cross section is to estimate what percentage of events used in the cross section calculation is not a primary pion. We start by noting that the last type of background, the "matched pile up" events, is a negligible fraction, because of the definition of the WC2TPC match: we deem the probability of a single match with a halo particle in the absence of a beamline particle $2^{2}$ negligibly small. As shown in Section 5.2.1, we use G4Beamline to estimate the percentage of pions, muons and electrons at $\mathrm{WC} 4$, obtaining the composition shown in Table 5.2. The next step is to simulate those pions, muons and electrons from WC4 to the TPC with the DDMC and evaluate their contribution to the cross section. To do so, we start by generating the same number of electrons, muons and pions with the DDMC and we apply the same selection chain applied in data (i.e. track multiplicity rejection, WC2TPC match and shower rejection) on the three samples. The number of events per particle species surviving this selection is shown on Table 5.3. In order to reproduce the data beamline composition, we weight each event of a given particle species according to the estimated content for that

2. Events with multiple WC2TPC matches are always rejected. 


\begin{tabular}{|l|l|l|l|l|l|l|}
\hline & \multicolumn{3}{|c|}{ Magnet Current -60A } & \multicolumn{3}{|c|}{ Magnet Current -100 A } \\
& MC $\pi^{-}$ & MC $\mu^{-}$ & MC $e^{-}$ & MC $\pi^{-}$ & MC $\mu^{-}$ & MC $e^{-}$ \\
\hline & & & & & & \\
Total Initial Events & 334500 & 334500 & 334500 & 344500 & 344500 & 344500 \\
Pass Multiplicity Filter & 330668 & 333420 & 198065 & 326576 & 344208 & 201380 \\
After WC2TPC Selection & 218239 & 296333 & 91139 & 230418 & 300228 & 98834 \\
Pass Shower Filter & 208063 & 288914 & 20293 & 219882 & 293585 & 17780 \\
& & & & & & \\
\hline & & & & & & \\
Selection Survival Rate & $62.2 \%$ & $86.4 \%$ & $6.1 \%$ & $63.8 \%$ & $85.2 \%$ & $5.2 \%$ \\
Beam Composition @WC4 & $68.8 \%$ & $4.6 \%$ & $26.6 \%$ & $87.4 \%$ & $3.7 \%$ & $8.9 \%$ \\
Expected Composition & $88.5 \%$ & $8.2 \%$ & $3.3 \%$ & $94.0 \%$ & $5.3 \%$ & $0.7 \%$ \\
in XS sample & & & & & & \\
\hline
\end{tabular}

Table 5.3: MC selection flow per particle species.

species as found with the G4Beamline simulation. In case of 60A runs, for example, the weights are 0.688 for pions, 0.046 for muons and 0.266 for electrons.

It should be noted that pions may interact hadronically in the steel (cryostat wall) or in the non-instrumented argon upstream to the TPC front face while traveling the length of between WC4 and the TPC. Or, they could decay in flight between WC4 and the TPC. One of the interaction or decay products can leak into the TPC and be matched with the WC track, contributing to the pool of events used for the cross section calculation. We call these occurrences "secondaries" from pion events, with a terminology inspired by Geant4. We estimate the number of secondaries using the DDMC pion sample. The percentage of secondaries is given by the number of matched WC2TPC tracks whose corresponding particle is not flagged as primary by Geant 4 . The secondary particles to primary pion ratio is $4.9 \%$ in the $60 \mathrm{~A}$ sample and $4.3 \%$ in the $100 \mathrm{~A}$ sample.

We evaluate the beamline background contribution to the cross section by producing the interacting and incident histograms for the signal and background events surviving the selection, staggering the contributions for each particle species, as shown in Figure 5.9 for the $-60 \mathrm{~A}$ case and in Figure 5.10 for the $-100 \mathrm{~A}$ case. From those 

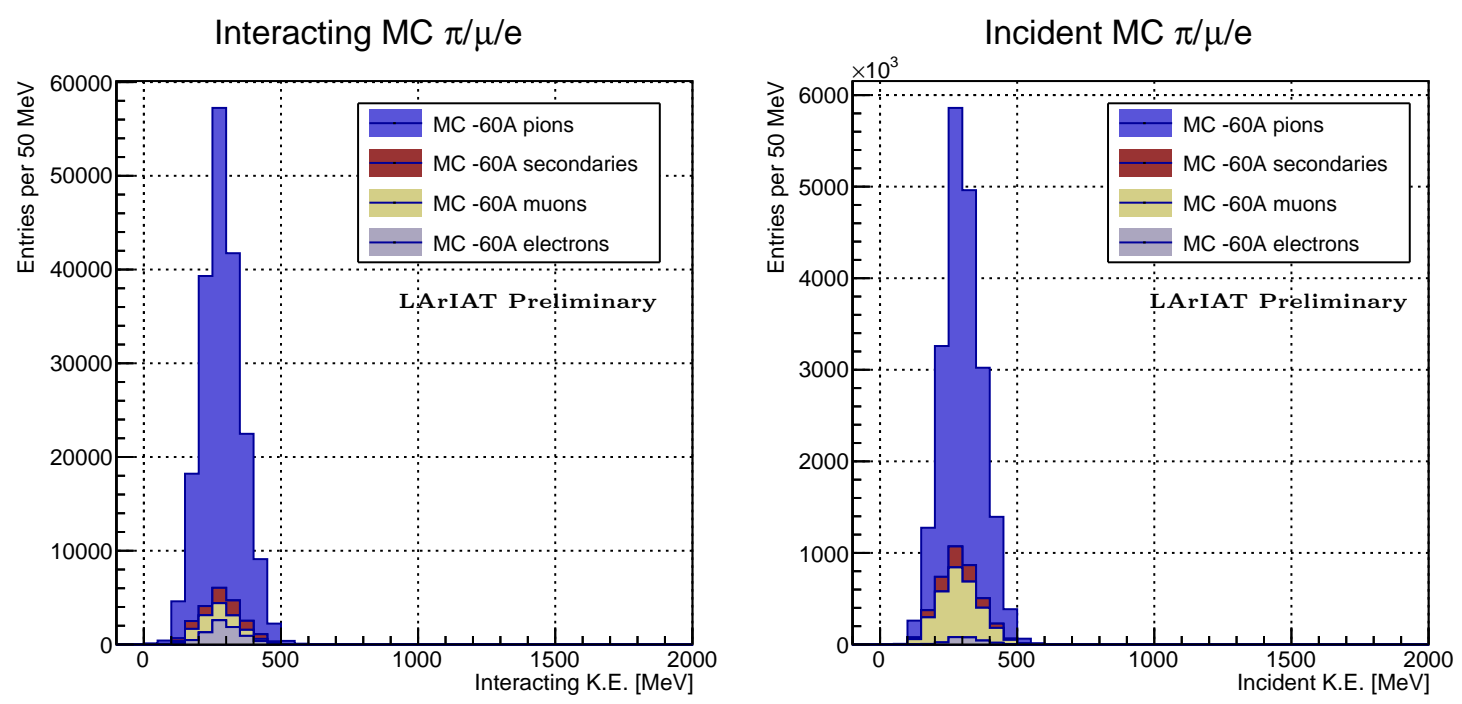

Figure 5.9: Left: staggered contributions to the interacting kinetic energy distribution for electron (grey), muons (yellow) and pion (blue) in the 60A simulation sample. Right: staggered contributions to the incident kinetic energy distribution for electron (grey), muons (yellow) and pion (blue) in the 60A simulation sample.
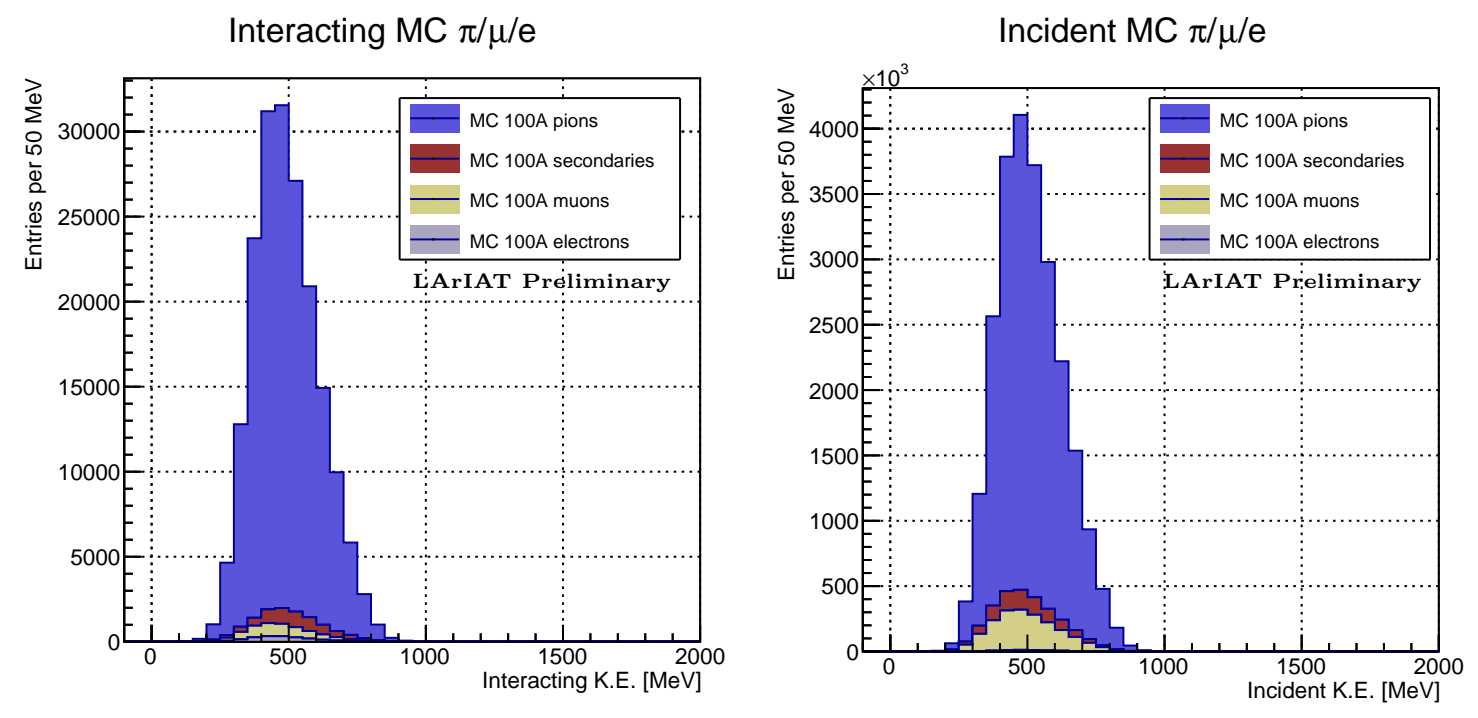

Figure 5.10: Left: staggered contributions to the interacting kinetic energy distribution for electron (grey), muons (yellow) and pion (blue) in the 100A simulation sample. Right: staggered contributions to the incident kinetic energy distribution for electron (grey), muons (yellow) and pion (blue) in the 100A simulation sample. 
histograms, we are able to evaluate the contribution of pions and beamline backgrounds to each bin of the interacting and incident histograms separately and obtain the relative pion content. The relative pion content in each bin for the interacting and incident histograms represents the correction applied to data. We take here the interacting histogram as example, noting that the derivation of the correction for the incident histogram is identical. The number of entries in each bin of the interacting histogram (Figure 5.9 left) is $N_{\text {Int }}^{\text {TOT }}\left(E_{i}\right)$, equal to the sum of the pions and beamline backgrounds flagged as interacting in that bin, namely

$$
N_{\text {Int }}^{\mathrm{TOT}}\left(E_{i}\right)=N_{\mathrm{Int}}^{\pi}\left(E_{i}\right)+\underbrace{N_{\mathrm{Int}}^{\mu}\left(E_{i}\right)+N_{\mathrm{Int}}^{e}\left(E_{i}\right)+N_{\mathrm{Int}}^{\text {Secondary }}\left(E_{i}\right)}_{B_{\mathrm{Int}}\left(E_{i}\right)} .
$$

Thus, the relative pion content to each bin in MC can be calculated as follows

$$
C_{\mathrm{Int}}^{\pi M C}\left(E_{i}\right)=\frac{N_{\mathrm{Int}}^{\pi M C}}{N_{\mathrm{Int}}^{T O T M C}\left(E_{i}\right)}=\frac{N_{\mathrm{Int}}^{T O T M C}\left(E_{i}\right)-B_{\mathrm{Int}}^{M C}\left(E_{i}\right)}{N_{\mathrm{Int}}^{T O T M C}\left(E_{i}\right)} .
$$

In order to evaluate the pion content of each bin in data, we scale the measured bin by the corresponding relative pion content found in $\mathrm{MC}$, as follows

$$
N_{\mathrm{Int}}^{\pi \text { RecoData }}=N_{\mathrm{Int}}^{\text {TOTData }}\left(E_{i}\right)-B_{\mathrm{Int}}^{\text {Data }}\left(E_{i}\right)=C_{\mathrm{Int}}^{\pi M C}\left(E_{i}\right) N_{\mathrm{Int}}^{\text {TOTData }}\left(E_{i}\right) .
$$

The pion content is evaluated separately in the interacting and incident histograms. Their ratio determines a correction to the measured raw cross section. For example, the measured raw cross section of a sample with enhanced muons content will tend to be lower than the raw cross section of a muon free sample. This is because most of the muons will cross the TPC without stopping, thus contributing almost exclusively to the incident histogram, forcing the pion content to be lower in the incident histogram than in the interacting; thus, the correction will tend to enhance the cross section. We present the estimation of $C_{\operatorname{Int}}^{\pi M C}\left(E_{i}\right)$ and $C_{\operatorname{Inc}}^{\pi M C}\left(E_{i}\right)$ and 
their associated systematic uncertainty in chapter discussing the pion cross section measurement (Figure 6.4.

\subsection{Estimate of Energy Loss before the TPC}

The beamline particles travel a path from where their momentum is measured in the beamline until they are tracked again inside the TPC. In the LArIAT geometry, a particle leaving the WC4 will encounter the materials listed in Table 5.4 before being registered again. The energy lost by the particle in this non-instrumented material modifies the particle's kinetic energy and directly affects the cross section measurement, as shown in equation 4.5 .

\begin{tabular}{|l|l|l|}
\hline Material & density $\left[\mathrm{g} / \mathrm{cm}^{3}\right]$ & width $[\mathrm{cm}]$ \\
\hline Fiberglass laminate $(\mathrm{G} 10)$ & 1.7 & 1.28 \\
Liquid Argon & 1.4 & 3.20 \\
Stainless Steel & 7.7 & 0.23 \\
Titanium & 4.5 & 0.04 \\
Air & $1.2 \cdot 10^{-3}$ & 89.43 \\
Plastic Scintillator & 1.03 & $1.20(+1.30)$ \\
\hline
\end{tabular}

Table 5.4: LArIAT material budget from WC4 to the TPC Front Face.

We derive an estimate of the energy loss between the beamline momentum measurement and the TPC $\left(E_{\text {loss }}\right)$ from the pion and kaon DDMC samples, since this quantity is not measurable directly on data. The $E_{\text {loss }}$ distribution for the $60 \mathrm{~A}$ and 100A pion sample is shown in figure 5.11, left and right respectively. The $E_{\text {loss }}$ distribution for the whole kaon sample is shown in figure 5.12. A clear double peaked structure is visible. After a long investigation on the origin of this unexpected shape, we determined that its origin is due to the particles either missing or hitting the HALO paddle (see Section 3.2.4 for the HALO paddle description): a schematic rendering of this occurrence is shown in figure 5.13. The kinematic at WC4 determines the trajectory of a particle and indicates whether or not it will hit the halo paddle, 
whose positioning and central hole size were not sufficiently well defined when the beamline instrumentation was installed. In Figure 5.14, we plot the true horizontal component of the momentum $P_{x}$ versus the true $X$ position at WC4 for pions missing the halo paddle (left) and for pions hitting the halo paddle (right) for the $-60 \mathrm{~A}$ MC simulation runs - analogous plots are obtained with the $-100 \mathrm{~A}$ pion simulation and with the kaon simulation. These distributions can be separated by a line in this position-momentum space. We use a logistic regression [1] as a classifier to find the best separating line, shown in both plots as the red line. We classify as "hitting the halo paddle" all pions whose $P_{x}$ and $X$ are such that

$$
P_{x}+0.02 * X-0.4<0
$$

and as "missing the halo paddle" all pions whose $P_{x}$ and $X$ are such that

$$
P_{x}+0.02 * X-0.4>0,
$$

where the coefficients of the line are empirically found by the logistic regression estimation. Overall, this simple method classifies in the right category (hit or miss) about $86 \%$ of the pion events. In MC, we assign $E_{\text {loss }}=32 \pm 4 \mathrm{MeV}$ for pion events classified as "hitting the halo paddle"; we assign $E_{\text {loss }}=24 \pm 3 \mathrm{MeV}$ for pion events classified as "missing the halo paddle". These values are the average and width of the two Landau distributions underneath the double peaked distribution. We apply the same classifier on data.

A late scan of the simulated geometry showed an excess of $3 \mathrm{~cm}$ of uninstrumented argon compared with the surveyed detector geometry. This excess has an effect on the $E_{\text {loss }}$ : MC particles traverse more uninstrumented material (i.e. loose more energy in the argon before the TPC front face) than data particles. We account for this difference by assigning in data $E_{\text {loss }}=24 \pm 6 \mathrm{MeV}$ for pion events classified as "hitting 
the halo paddle" and $E_{\text {loss }}=17 \pm 6 \mathrm{MeV}$ for pion events classified as "missing the halo paddle", where the uncertainty is derived as the standard deviation of the double peaked distribution.

The summary of the values for used for $E_{\text {Loss }}$ for the pion sample is listed in Table 5.5 with the analogous results for the study on the kaon case.
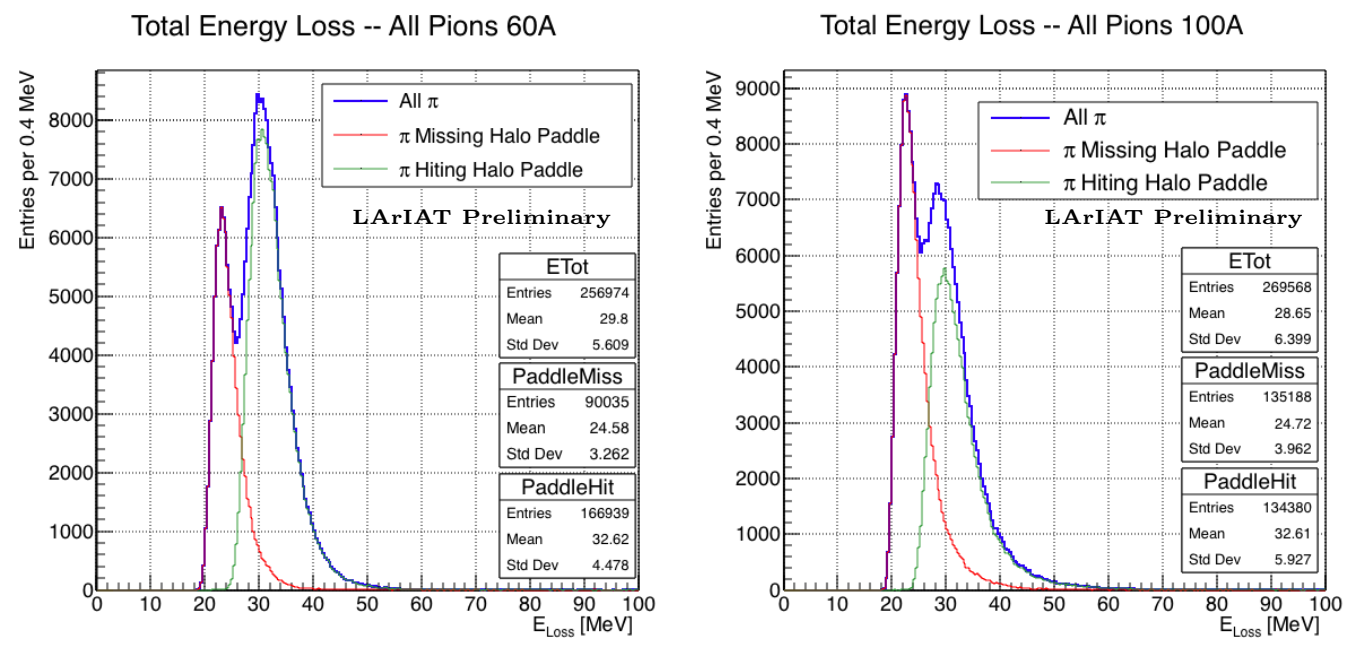

Figure 5.11: True energy loss between $\mathrm{WC} 4$ and the TPC front face according to the MC simulation of negative pions of the $60 \mathrm{~A}$ runs (left) and of the $100 \mathrm{~A}$ runs (right). The distribution for the whole data sample is shown in blue, the distribution for the pions missing the halo is shown in red, and the distribution for the pions hitting the halo is shown in green.

\subsection{Tracking Studies}

The tracking of hadrons in the TPC determines both the beamline to TPC handshake and the identification of the interaction point within the TPC. Thus, it plays

\begin{tabular}{|l|c|c|}
\hline & \multicolumn{2}{|c|}{$\mathrm{E}_{\text {loss }}[\mathrm{MeV}]$} \\
\hline & Hitting Halo & Missing Halo \\
\hline Pion MC & $32 \pm 4$ & $24 \pm 3$ \\
\hline Pion Data & $25 \pm 6$ & $17 \pm 6$ \\
\hline Kaon MC & $38 \pm 6$ & $31 \pm 5$ \\
\hline Kaon Data & $26 \pm 7$ & $22 \pm 7$ \\
\hline
\end{tabular}

Table 5.5: Energy loss for pions and kaons. 


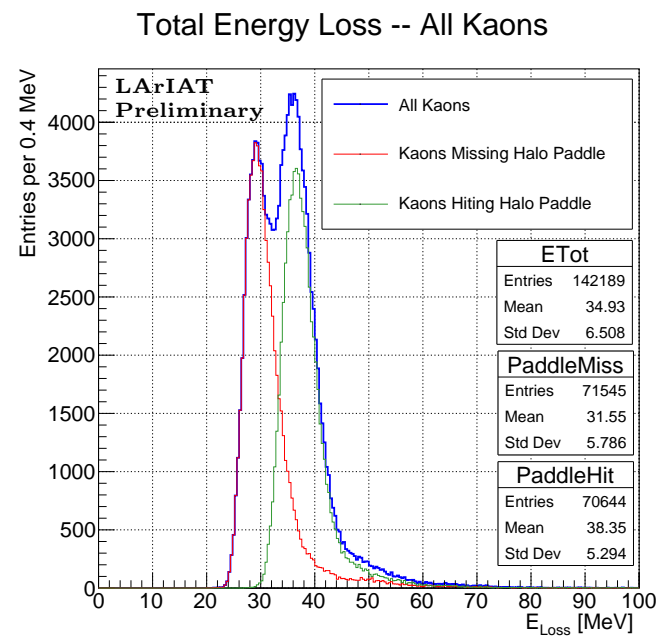

Figure 5.12: True energy loss between WC4 and the TPC front face according to the $\mathrm{MC}$ simulation of positive kaons in the $60 \mathrm{~A}$ and $100 \mathrm{~A}$ combined sample. The distribution for the whole data sample is shown in blue, the distribution for the kaons missing the halo is shown in red, and the distribution for the kaons hitting the halo is shown in green.

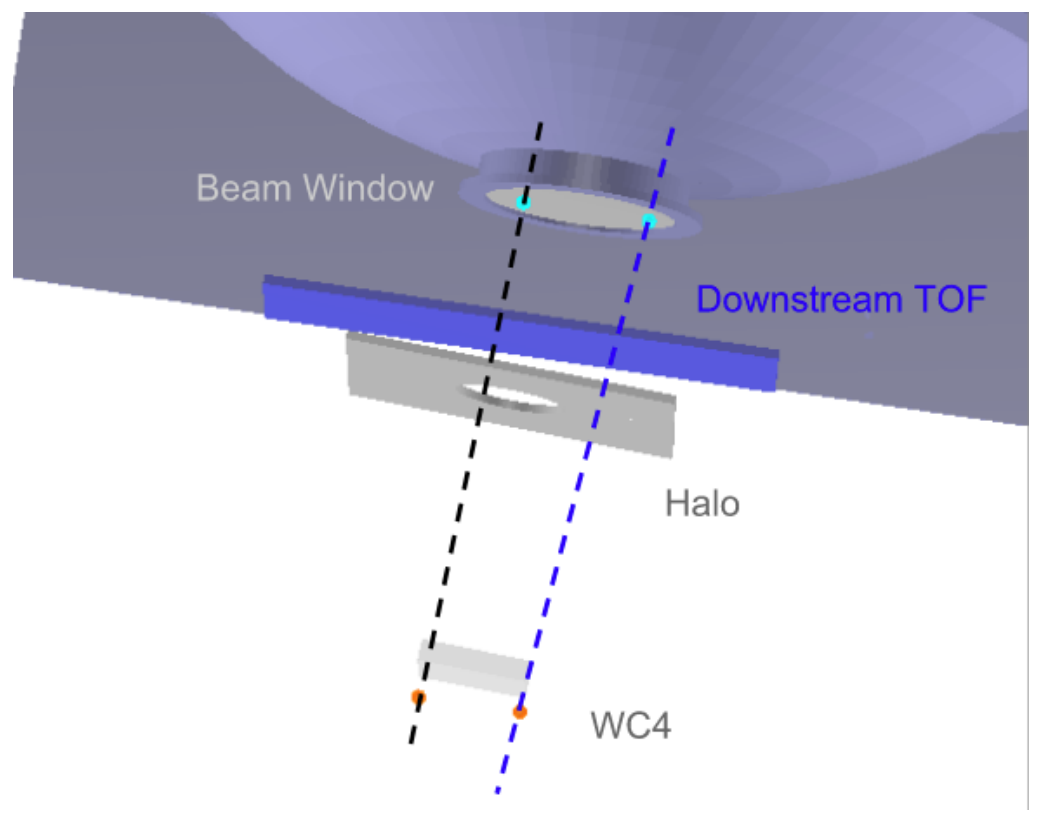

Figure 5.13: Schematic rendering of the particle path between WC4 and the TPC front face. The paddle with the hollow central circle represents the Halo paddle. We illustrate two possible trajectories: in black, a trajectory that misses the paddle and goes through the hole in the Halo, in blue a trajectory that hits the Halo paddle and goes through the scintillation material. 

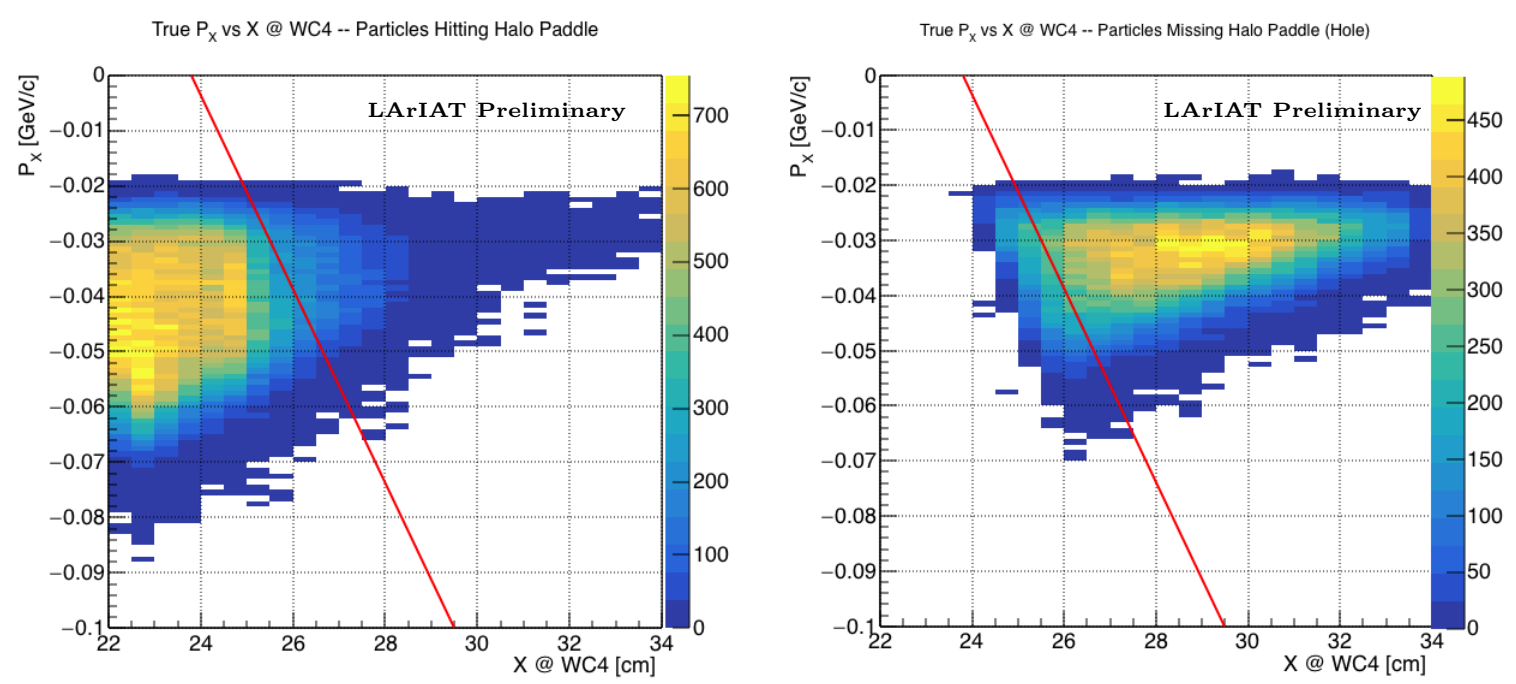

Figure 5.14: Horizontal component of the true momentum vs the horizontal position at WC4 for $\mathrm{MC}$ simulated pions of the 60A runs. The plot on the left shows the distribution for pion that miss the halo paddle and the plot on the right shows the distributions for pions that hit the halo. The form of the classifier is overlaid to both plots (red line).

a fundamental role in the cross section measurements. We performed several studies geared towards the optimization of the LArSoft software package for tracking in the TPC. In particular, we studied a suitable set of parameters for the WC2TPC match and we optimized the clustering algorithm to maximize the efficiency of finding the interaction point on MC. Given the technical nature of these studies, we report them in Appendix B. We only report here the evaluation of the angular resolution of the tracking algorithm and a study of the track pitch in data and MC, due to their important implications on the physics measurement.

\subsubsection{Angular Resolution}

The scope of this study is to understand and compare the tracking performances and the angular resolution of the TPC tracking on data and MC. We use the angular resolution of the tracking to determine the value of smallest angle that the tracking algorithms allow to reconstruct with a non-zero efficiency, effectively determining a 
selection on the distribution of the scattering angle of hadronic interaction entering the cross section measurement.

We start by selecting all the WC2TPC matched tracks used for the cross section analysis. These tracks can contain from a minimum of $33 \mathrm{D}$-space points to a maximum of 240 3D-space points. We fit a straight line to all the 3D-space points associated with the track. For each track we calculate the average distance between each point in space and the fit line as follows

$$
\bar{d}=\frac{\sum_{i}^{N} d_{i}}{N}
$$

where $N$ is the number of 3D-space points of the track and $d_{i}$ is the distance of the $i$-th space point to the line fit. Several tests to compare the goodness of fit between data and MC have been considered. We decided to use $\bar{d}$ for its straightforward interpretation. The $\bar{d}$ distribution for data and MC is shown in Figure 5.17 for pions and in Figure 5.19 for kaons and shows a relatively good agreement between data and MC.

A visual representation of the procedure used to evaluate the angular resolution is shown in Figure 5.15. For each track, we order the space points according to their Z position along the positive beam direction (panel a) and we split them in two sets: the first set contains all the points belonging to the first half of the track and the second set contains all the points belonging the second half of the track. We remove the last four points in the first set and the first four points in the second set, so to have a gap in the middle of the original track (panel b). We fit the first and the second set of points with two lines (panel c). We then calculate the angle between the fit of the first and second half $\alpha$ (panel d). The angle $\alpha$ determines the angular resolution of the tracking. The distributions for data and $\mathrm{MC}$ for $\alpha$ are given in Figure 5.18 for pions and in Figure 5.20 for kaons. The mean of the data and MC angular resolution 
are reported in Table 5.6 for pions and kaons in data and MC.

Interaction angles smaller than the angular resolution are indistinguishable for the reconstruction. Therefore, we assess our ability to measure the cross section to be limited to interaction angles greater than $5.0 \mathrm{deg}$. More accurate studies of the angular resolution as a function of the kinetic energy and track length, albeit interesting, are left for an improvement of the analysis.

As we discussed in Section 1.4.1, several different interaction topologies are included as signal in the total hadronic cross section. The ability to detect a minimum interaction angle and to stop the tracking accordingly mainly effects two interaction channels: the pion elastic interaction (see Table 1.4, second line) and the pion inelastic interaction in case of neutral particle emission (see Table 1.4, fourth line); the overall effect of this limitation is to reduced the cross section measurement to the measurement of the cross section relative to interaction angles greater than a $\sim 5.0$ deg . It is beneficial to take a moment to describe the definition of interaction angle. In case of elastic scattering, the definition is straightforward: the interaction angle is the angle between the incoming and outgoing hadron, i.e.

$$
\theta=\cos ^{-1}\left(\frac{\vec{p}_{\text {incoming }} \cdot \vec{p}_{\text {outgoing }}}{\left|\vec{p}_{\text {incoming }}\right|\left|\vec{p}_{\text {outgoing }}\right|}\right)
$$

In case of the reaction channel, the presence of several topologies requires a more complex definition, as shown in Figure 5.16. We define the scattering angle as the biggest of the angles between the incoming hadron and the visible daughters, where the visible daughters are charged particles that travel more than the average pitch length $(\delta X=47 \mathrm{~mm})$ in the detector (see panel a); in case all the daughters are

\begin{tabular}{|l|c|c|}
\hline & Data & MC \\
\hline Pions & $\bar{\alpha}_{\text {Data }}=(5.0 \pm 4.5) \mathrm{deg}$ & $\bar{\alpha}_{M C}=(4.5 \pm 3.9) \mathrm{deg}$ \\
\hline Kaons & $\bar{\alpha}_{\text {Data }}=(4.3 \pm 3.7) \mathrm{deg}$ & $\bar{\alpha}_{M C}=(4.4 \pm 3.6) \mathrm{deg}$ \\
\hline
\end{tabular}

Table 5.6: Angular resolution for Pion and Kaon tracking in both data and MC. 
invisible, the angle is assigned to be $90 \mathrm{deg}$ (see panel b). We chose this working definition of scattering angle for inelastic scattering keeping in mind how our tracking reconstruction works: the tracking will stop correctly if none of the daughters are visible in the detector and it is likely to stop correctly if multiple visible daughters form an interaction vertex. The only "dangerous" case is the production of one charged daughter plus neutrals, which we can study with this working definition of scattering angle (see panel c).

Once we fix the scattering angle definition, we can study the effects of the angular resolution on the cross section by plotting the true Geant 4 total hadronic cross section for interaction angles greater than a minimum interaction angle. The left side of Figure 5.21 shows the true Geant 4 cross section for interaction angles greater than 0 deg (green), $4.5 \mathrm{deg}$ (red) corresponding to the $\mathrm{MC}$ angular resolution, $5.0 \mathrm{deg}$ (blue) corresponding to the data angular resolution, and 9.0 deg (yellow). As reported in Table 5.6, we find a 0.5 deg difference between the mean of the data and MC angular resolution, which has a negligible impact on the cross section. The right side of Figure 5.21 shows the ratio between the true cross section for interaction angles greater than 5 deg and the true interaction cross section for all angles; the cross section for angles greater than $5^{\circ}$ accounts for more than $80 \%$ than the total cross section in every energy bin.

\subsubsection{Track Pitch}

The granularity of the LArTPC detector leads to the fine-sampling of a hadron inside the argon active volume. This detector granularity translates into the "track pitch" quantity, which is the distance between two consecutive 3D points of the tracking. The track pitch determines the width of the slab $\delta X$ in the cross section Equation 4.7. Figure 5.22 shows the pitch distribution in data (black) and MC (red) for the pion combined sample on the left and the kaon sample on the right. The MC and 


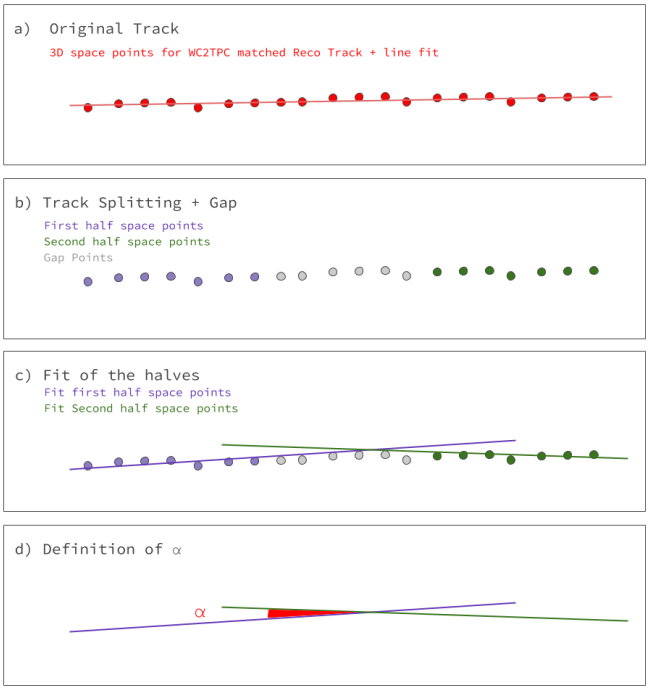

Figure 5.15: A visual representation of the procedure used to evaluate the angular resolution.
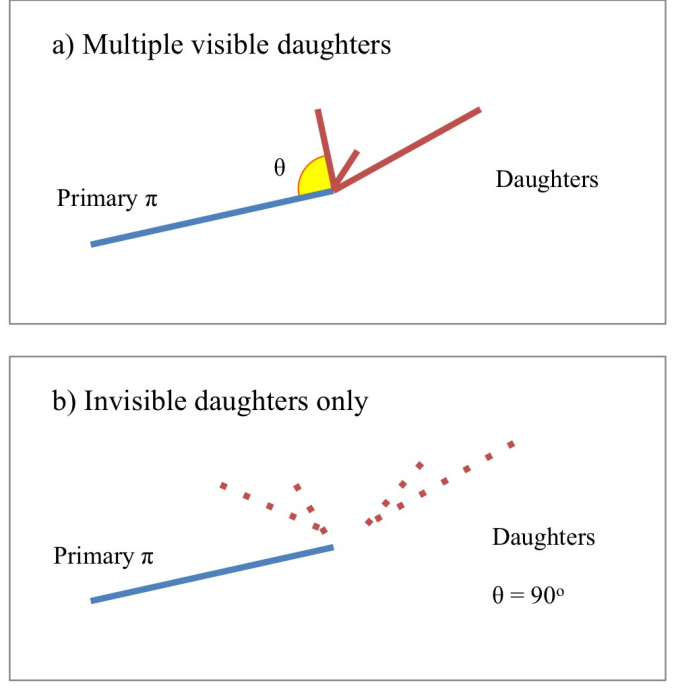

c) Visible and invisible daughters

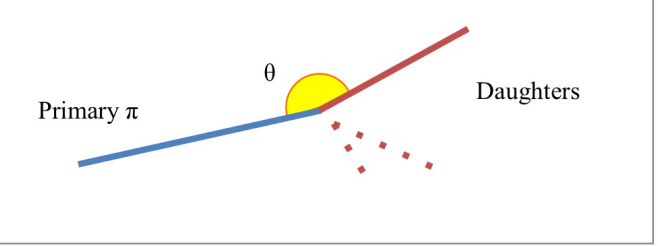

Figure 5.16: A visual representation of the scattering angle definition in case of inelastic scattering.

data distributions agree rather well. Thus, we estimate the slab width to be $\delta X=$ $0.47 \pm 0.03 \mathrm{~cm}$. 


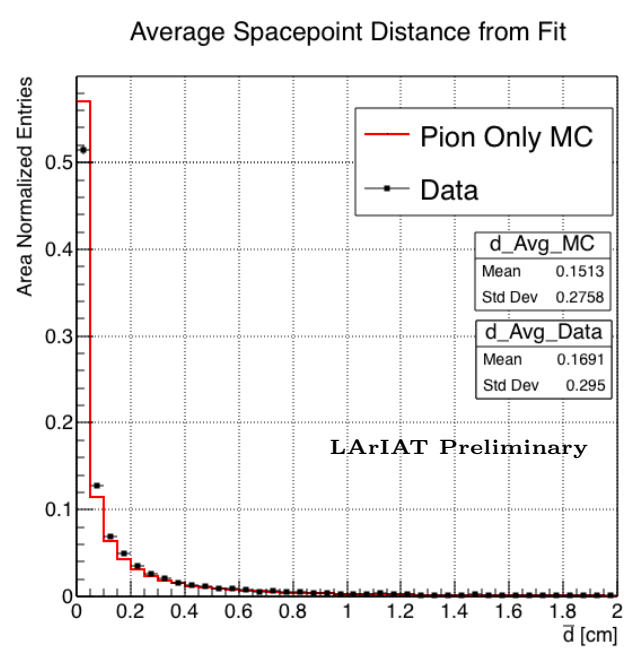

Figure 5.17: Distributions of the average distance between each 3D point in space and the fit line, $\bar{d}$ for the data used in the pion cross section analysis and the pion only DDMC. The distributions are area normalized.

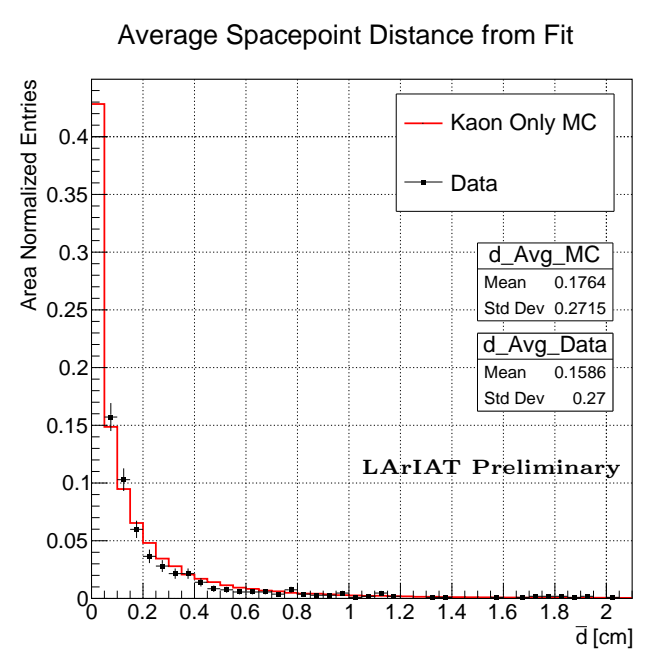

Figure 5.19: Distributions of the average distance between each $3 \mathrm{D}$ point in space and the fit line, $\bar{d}$ for the data used in the kaon cross section analysis and the kaon only DDMC. The distributions are area normalized.

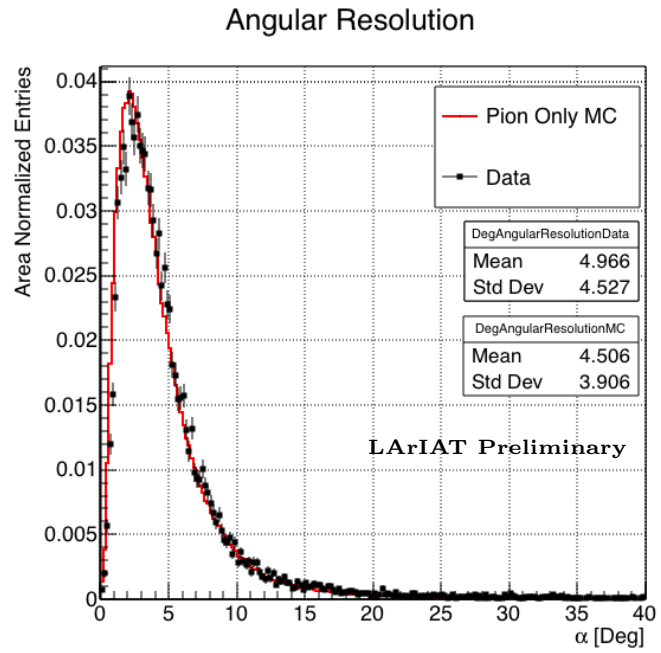

Figure 5.18: Distributions of angular resolution $\alpha$ for data used in the pion cross section analysis and pion only DDMC. The distributions are area normalized.

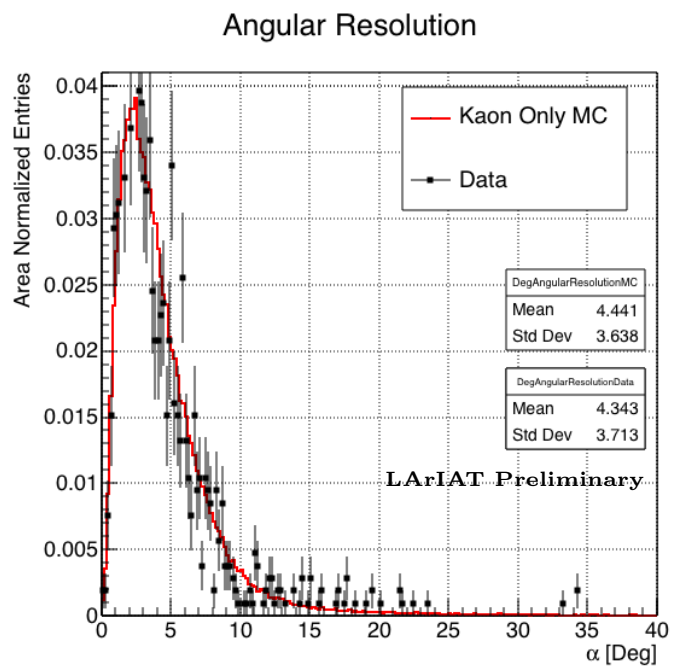

Figure 5.20: Distributions of angular resolution $\alpha$ for data used in the kaon cross section analysis and kaon only DDMC. The distributions are area normalized. 
Geant4 ( $\left.\pi^{-}, \mathrm{Ar}\right)$ True Cross Section

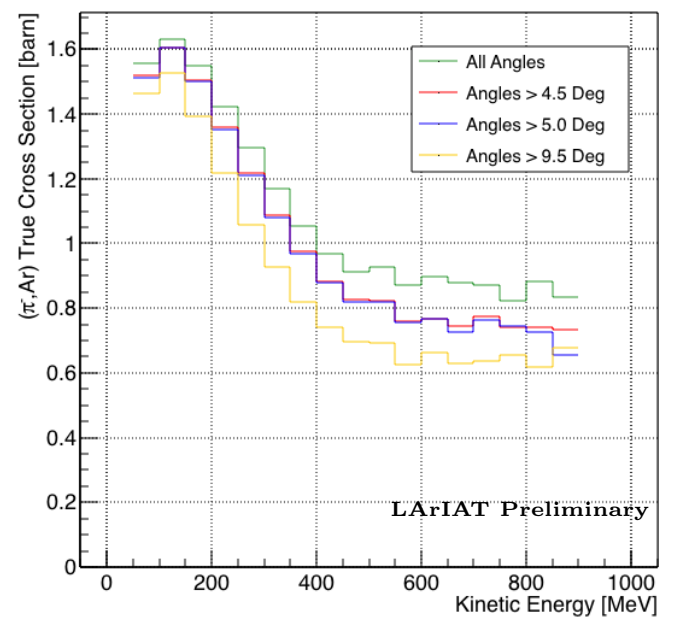

Percentage of Cross Section with Interaction Angle > 5 Deg

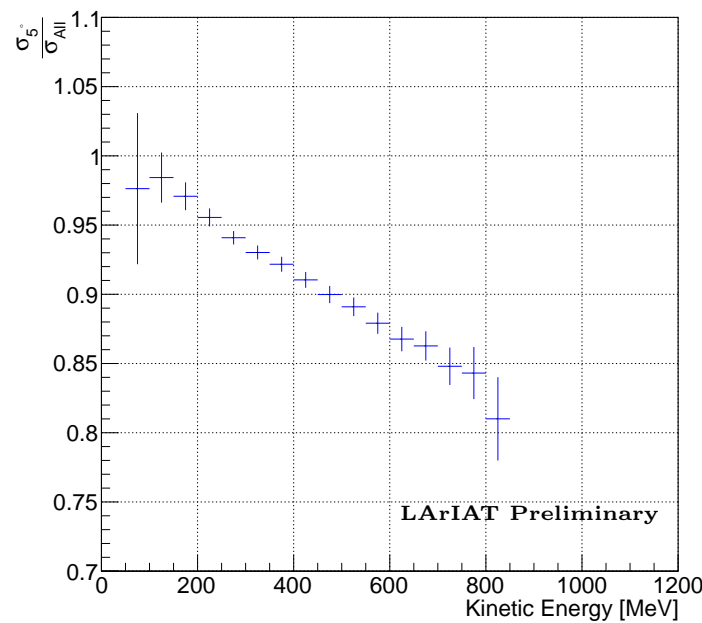

Figure 5.21: Left: True $\left(\pi^{-}, A r\right)$ cross section for interaction angles greater than 0 deg (green), $4.5 \mathrm{deg}$ (red), $5.0 \mathrm{deg}$ (blue) and $9.0 \mathrm{deg}$ (yellow). Right: Ratio between the true cross section for interaction angles greater than $5 \mathrm{deg}$ and the true interaction cross section for all angles.

Track Pitch

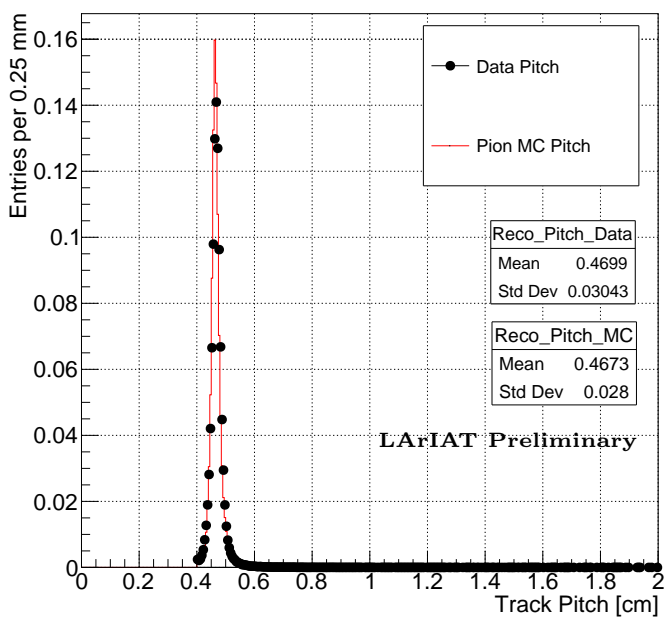

Track Pitch

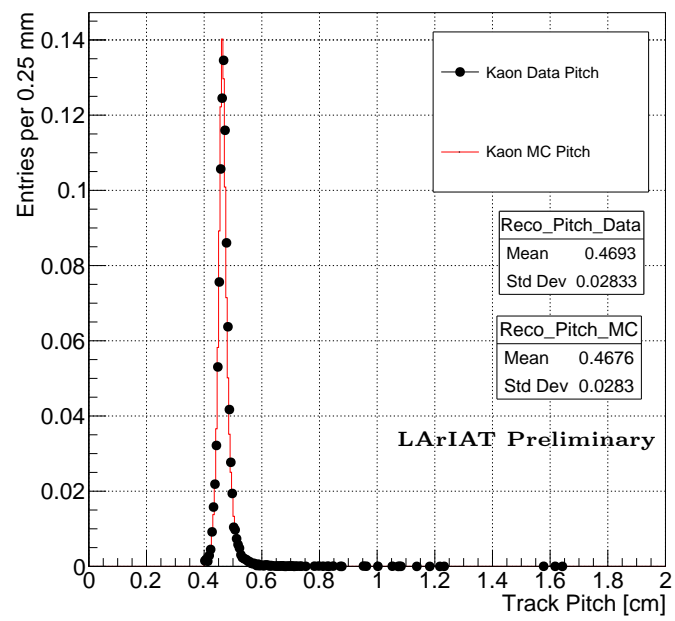

Figure 5.22: Left: Pitch distribution for the pion combined sample 60A and 100A, data displayed in black, MC in red. Right: Pitch distribution for the kaon sample, data displayed in black, $\mathrm{MC}$ in red. 


\subsection{Calorimetry Studies}

The measured kinetic energy of a hadron candidate at each argon slab determines which bins of the interacting and incident histograms a selected event is going to fill. Thus, the energy measurement provided by the LArTPC is fundamental for the cross section analysis. In Appendix C, we describe how we calibrate the TPC calorimetric response. In this section, we describe how we measure the kinetic energy of the hadrons in the TPC.

\subsubsection{Kinetic Energy Measurement}

In this section, we define the measurement on the kinetic energy and determine the related uncertainty. We will propagate this uncertainty into the cross section measurement, as discussed in Section 6.1.2 for the pion cross section and in Section 7.1 for the kaon cross section.

The kinetic energy of a hadron at the $j^{\text {th }}$ slice of argon in the TPC is given by

$$
K E_{j}=\sqrt{p_{\text {Beam }}^{2}+m_{\text {Beam }}^{2}}-m_{\text {Beam }}^{2}-E_{\text {Loss }}-E_{\mathrm{FF}-\mathrm{j}}
$$

where $p_{\text {Beam }}$ is the momentum measured by the beamline detectors, $m_{\text {Beam }}$ is the mass of the hadron as reported in the PDG, $E_{\text {Loss }}$ is the energy loss between the beamline and the TPC, and $E_{\mathrm{FF}-\mathrm{j}}$ is the energy that the hadron deposited from the TPC front face until the $j^{\text {th }}$ slice. The uncertainty on $K E_{j}$ is then given by

$$
\delta K E_{j}=\sqrt{\delta p_{\text {Beam }}^{2}+\delta E_{\text {Loss }}^{2}+\delta E_{\mathrm{dep} \mathrm{FF-j}}^{2}}
$$

where we have dropped the uncertainty on the mass, since it is orders of magnitude smaller than the other uncertainties. We assume the relative uncertainty on $p_{\text {Beam }}$ to be $2 \%$, and the uncertainty on the energy loss upstream to be $7 \mathrm{MeV}$, as calculated 
in Section 5.4. We describe the estimate of the uncertainty on $E_{\mathrm{FF}-\mathrm{j}}$ in the rest of this section.

The energy deposited by the hadron from the TPC front face until the $j^{\text {th }}$ slice is the sum of the measured energy deposited in each previous slabs $E_{i}$, i.e.

$$
E_{\mathrm{FF}-\mathrm{j}}=\sum_{i<j} E_{i}
$$

where $E_{i}$ is measured in each slab as the product of the stopping power, $d E / d X_{i}$, and the track pitch for that point. Since the measurements of the energy deposited in each slab rely on the same global calorimetric procedure and tracking algorithms, we assume conservatively that the measurements of $E_{i}$ are not independent from one another; thus, the uncertainty on $E_{\mathrm{FF}-\mathrm{j}}$ becomes

$$
\delta E_{\mathrm{FF}-\mathrm{j}}=\sum_{i<j} \delta E_{i}=(j-1) \delta E_{i}
$$

where $\delta E_{i}$ is the uncertainty on the energy loss in one slab of argon.

The left side of Figure 5.23 shows the distribution of the energy deposited in each slab of argon, for the 60A negative pion dataset in black and for the pion only MC in blue. The analogous plot for the $-100 \mathrm{~A}$ negative pion data set is show on the right side of Figure 5.23. The distributions are fitted with a Landau displayed in red for data and in teal for MC. The uncertainty on $E_{i}$ is given by the width of the Landau fit to the data. A small systematic uncertainty is given by a $1.0 \%$ difference between the most probable value of the Landau fits in data and MC. 

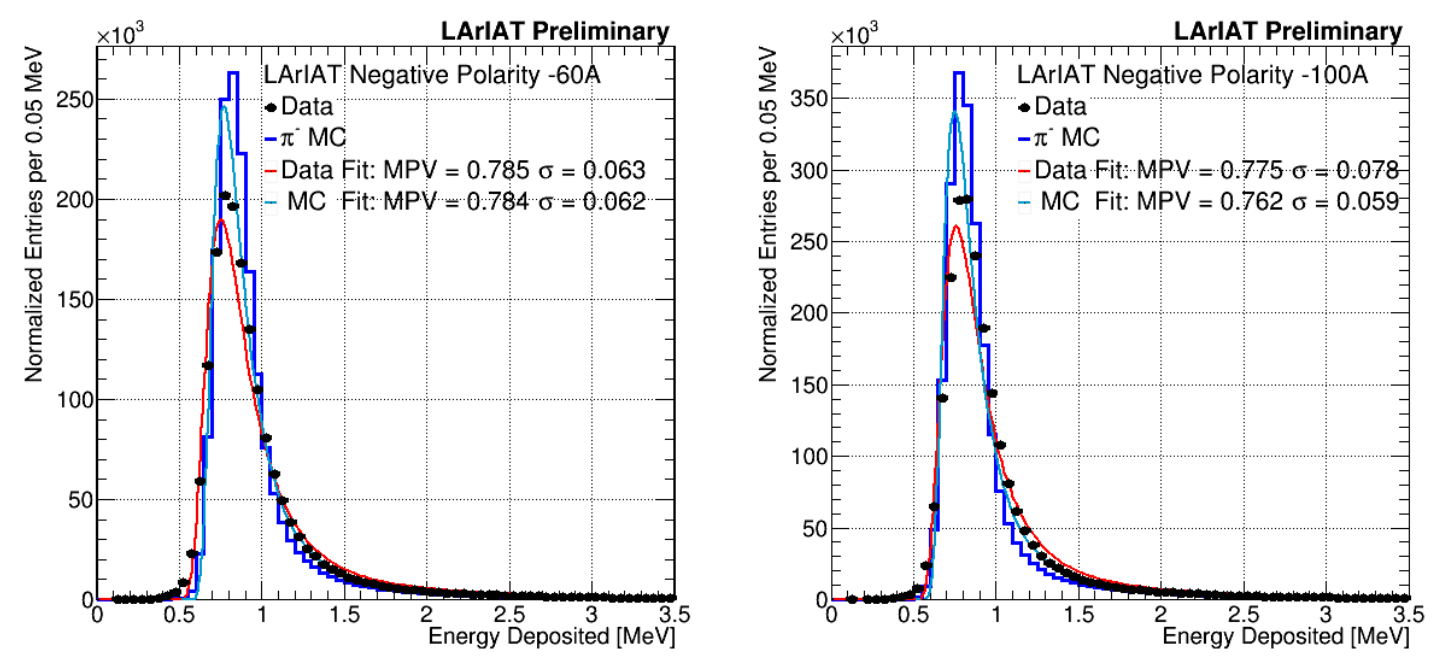

Figure 5.23: Energy deposited $E_{i}$ in a single slab of argon for the pion -60A runs (left) and -100A runs (right). The data is shown in black, the MC in blue. The distributions are fitted with a Landau displayed in red for data and in teal for MC.

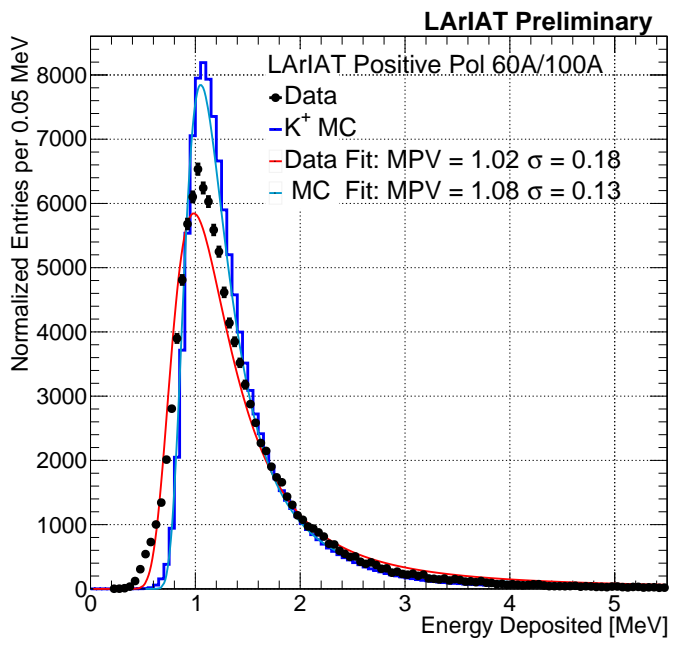

Figure 5.24: Energy deposited $E_{i}$ in a single slab of argon for the kaons of the $+60 \mathrm{~A}$ runs and $+100 \mathrm{~A}$ runs. The data is shown in black, the MC in blue. The distributions are fitted with a Landau displayed in red for data and in teal for MC. 


\section{Chapter 6}

\section{Negative Pion Cross Section}

\section{Measurement}

" $Y$ ella es flama que se eleva, $Y$ es un pájaro a volar.

En la noche que se incendia, estrella de oscuridad que busca entre la tiniebla, la dulce hoguera del beso."

- Lila Downs, 2002 -

In this chapter, we show the results of the thin slice method to measure the $\left(\pi^{-}\right.$Ar) total hadronic cross section. In Section 6.1, we start by measuring the raw cross section, i.e. the cross section obtained exclusively using data reconstruction, without any additional correction. In Section 6.2, we apply the statistical subtraction of the background contributions based on simulation and the correction for reconstruction effects. The final results are presented in Section 6.3.

\subsection{Raw Cross Section}

We measure the raw $\left(\pi^{-}-\mathrm{Ar}\right)$ total hadronic cross section as a function of the kinetic energy in the two chosen data sets, the $-60 \mathrm{~A}$ and $-100 \mathrm{~A}$ negative runs. As we will 
clarify in Section 6.2, the corrections to the raw cross section depend on the beam settings and need to be calculated independently for the two datasets. Thus, we present here the measurements of the raw cross section on the two datasets separately. As stated in Section 4.3.2, the raw cross section is given by the Equation 4.4

$$
\sigma_{T O T}\left(E_{i}\right)=\frac{1}{n \delta X} \frac{N_{\mathrm{Int}}^{\mathrm{TOT}}\left(E_{i}\right)}{N_{\mathrm{Inc}}^{\mathrm{TOT}}\left(E_{i}\right)},
$$

where $N_{\text {Int }}^{\mathrm{TOT}}$ is the measured number of particles interacting at kinetic energy $E_{i}$, $N_{\text {Inc }}^{\mathrm{TOT}}$ is the measured number of particles incident on an argon slice at kinetic energy $E_{i}, n$ is the density of the target centers and $\delta X$ is the thickness of the argon slice. The density of the target centers and the slab thickness are $n=0.021 \cdot 10^{24} \mathrm{~cm}^{-3}$ and $\delta X=0.47 \mathrm{~cm}$, respectively.

Figure 6.1 shows the distribution of $N_{\text {Int }}^{\mathrm{TOT}}$ as a function of the kinetic energy for the $60 \mathrm{~A}$ dataset on the left and for the $100 \mathrm{~A}$ dataset on the right. The data central points are represented by black dots, the statistical uncertainty is shown in black, while the systematic uncertainty is shown in red. Data is displayed over the $N_{\text {Int }}^{\text {TOT }}$ distribution obtained with a MC sample of pions, muon and electrons weighted by the beam composition (additional details on the composition will be provided in Section 6.2.1). The contribution from the simulated pions is shown in blue, the one from secondaries in red, the one from muons in yellow and the ones from electrons in gray. The simulated pion's and backgrounds' contributions are stacked; the sum of the integrals from each particle species is normalized to the integral of the data.

Figure 6.2 shows the distribution of $N_{\text {Inc }}^{\text {TOT }}$ for the $60 \mathrm{~A}$ dataset on the left and for the $100 \mathrm{~A}$ dataset on the right. Data is displayed over MC. The same color scheme and normalization procedure is used for both the interacting and incident histograms.

Figure 6.3 shows the raw cross section for the $60 \mathrm{~A}$ dataset on the left and for the 100A dataset on the right, statistical uncertainty in black and systematic uncertainty 
in red. The raw data cross section is overlaid to the reconstructed cross section for the MC sample, displayed in azure. Since the background contributions and the detector effects for the $60 \mathrm{~A}$ and $100 \mathrm{~A}$ sample are different, it is premature to compare the raw cross sections obtained from the two samples at this point.

We describe the calculation of the statistical uncertainty for the interacting, incident and cross section distributions in Section 6.1.1; we describe the procedure to calculate the corresponding systematics uncertainty on Section 6.1.2.

\subsubsection{Statistical Uncertainty}

The statistical uncertainty for a given kinetic energy bin of the cross section is calculated by error propagation from the statistical uncertainty on $N_{\text {Inc }}^{\mathrm{TOT}}$ and $N_{\text {Int }}^{\mathrm{TOT}}$ correspondent bin. Since the number of incident particles in each energy bin is given by a simple counting, we assume that $N_{\text {Inc }}^{\text {TOT }}$ is distributed as a poissonian with mean and variance equal to $N_{\text {Inc }}^{\text {TOT }}$ in each bin. On the other hand, $N_{\text {Int }}^{\text {TOT }}$ follows a binomial distribution: a particle in a given energy bin might or might not interact. The variance for the binomial is given by

$$
\operatorname{Var}\left[N_{\text {Int }}^{\mathrm{TOT}}\right]=\mathcal{N} P_{\text {Interacting }}\left(1-P_{\text {Interacting }}\right) .
$$

Since the interaction probability $P_{\text {Interacting }}$ is estimated by $\frac{N_{\text {Int }}^{\mathrm{TOT}}}{N_{\text {Inc }}^{\mathrm{DOT}}}$ and the number of tries $\mathcal{N}$ is $N_{\text {Inc }}^{\mathrm{TOT}}$, Equation 6.1 translates into

$$
\operatorname{Var}\left[N_{\text {Int }}^{\mathrm{TOT}}\right]=N_{\text {Inc }}^{\mathrm{TOT}} \frac{N_{\text {Int }}^{\mathrm{TOT}}}{N_{\text {Inc }}^{\mathrm{TOT}}}\left(1-\frac{N_{\text {Int }}^{\mathrm{TOT}}}{N_{\text {Inc }}^{\mathrm{TOT}}}\right)=N_{\text {Int }}^{\mathrm{TOT}}\left(1-\frac{N_{\text {Int }}^{\mathrm{TOT}}}{N_{\text {Inc }}^{\mathrm{TOT}}}\right) .
$$

$N_{\text {Inc }}^{\mathrm{TOT}}$ and $N_{\text {Int }}^{\mathrm{TOT}}$ are not independent. In fact, populating a given bin for the interacting histogram always implies at least populating the same bin in the incident histogram (and possibly other incidents bins at higher energies). Thus, we conserva- 

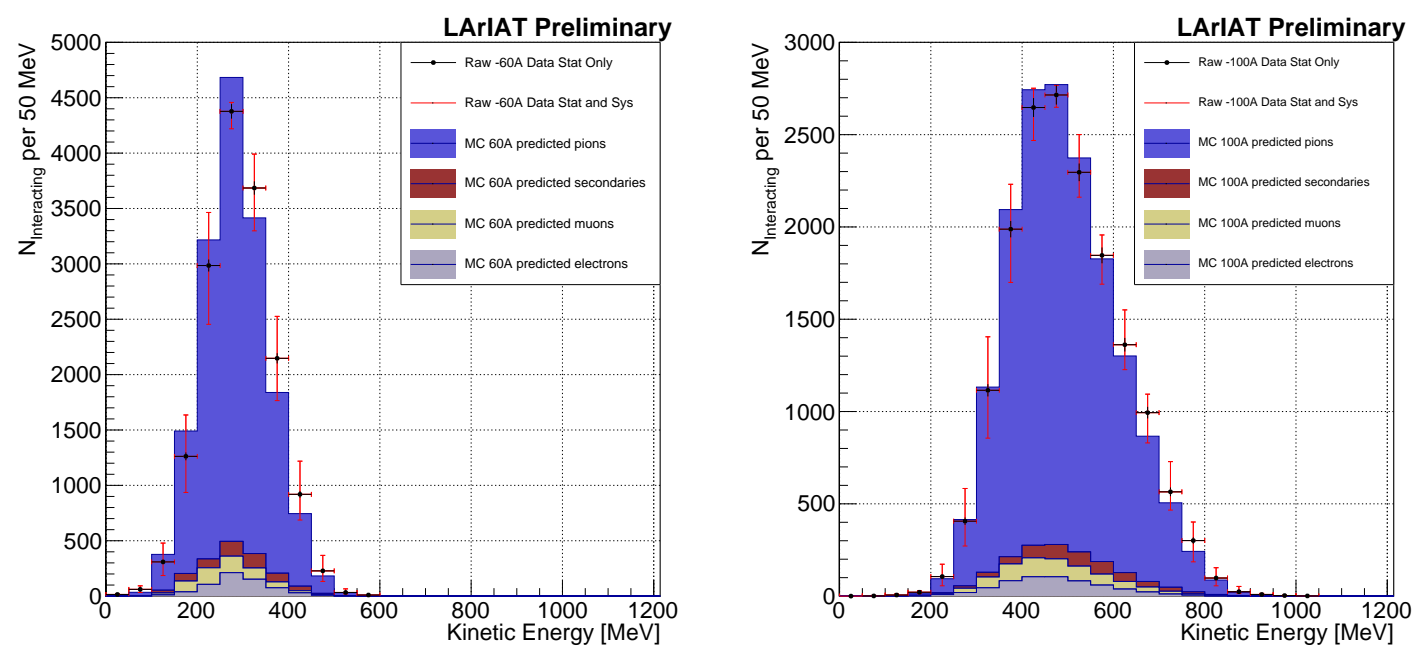

Figure 6.1: Raw number of interacting pion candidates as a function of the reconstructed kinetic energy for the $60 \mathrm{~A}$ runs (left) and for the $100 \mathrm{~A}$ runs (right). The statistical uncertainties are shown in black, the systematic uncertainties in red.
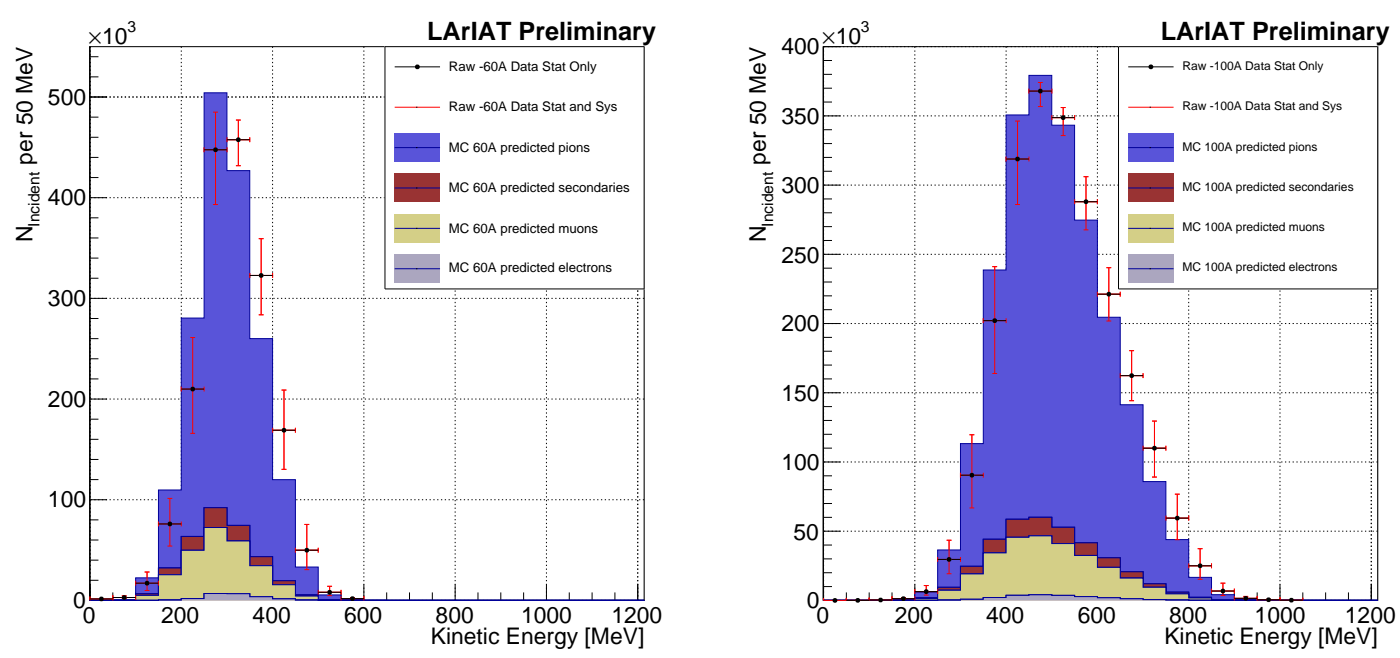

Figure 6.2: Raw number of incident pion candidates as a function of the reconstructed kinetic energy for the 60A runs (left) and for the 100A runs (right). The statistical uncertainty is shown in black, the systematic uncertainties in red. 

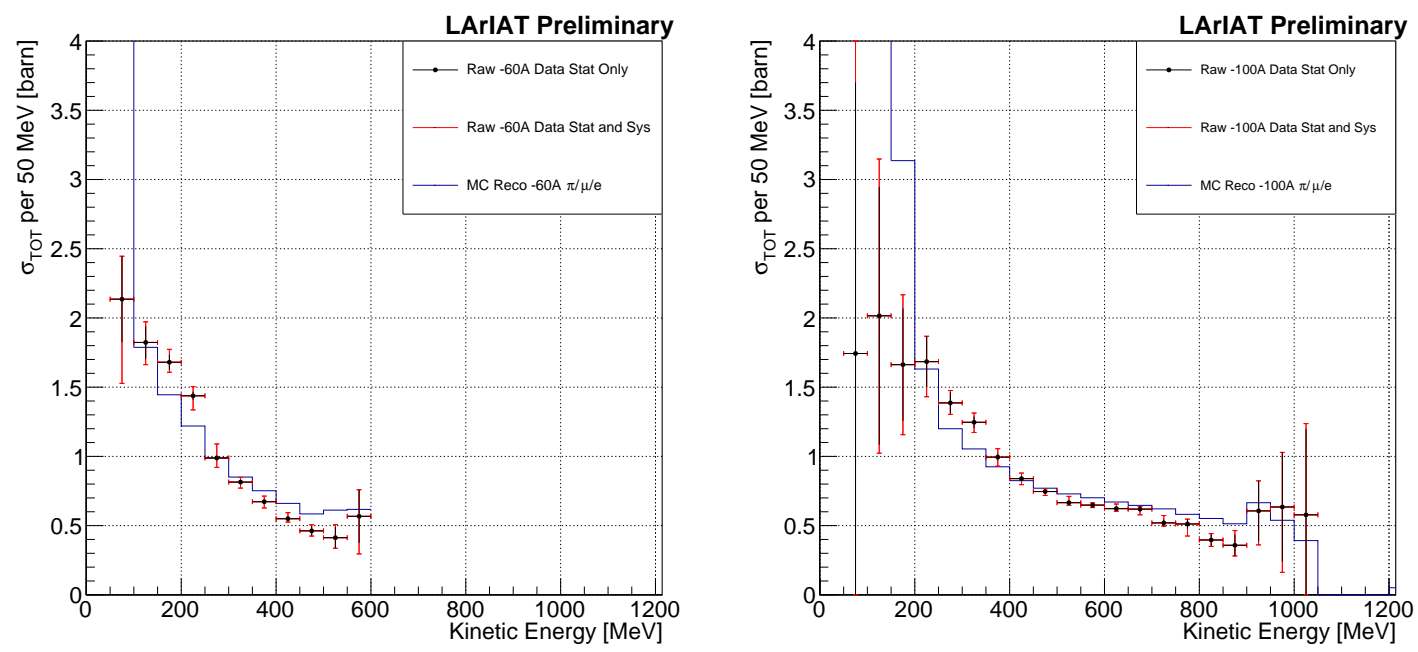

Figure 6.3: Raw $\left(\pi^{-}-\mathrm{Ar}\right)$ total hadronic cross section for the 60A runs (left) and for the 100A runs (right). The statistical uncertainty is shown in black, the systematic uncertainties in red. The raw cross section obtained with a MC mixed sample of pions, muon and electrons in the percentage predicted by G4Beamline is shown in azure.

tively calculate the statistical uncertainty on the cross section as

$$
\frac{\delta \sigma_{T O T}(E)}{\sigma_{T O T}(E)}=\left(\frac{\delta N_{\mathrm{Int}}^{\mathrm{TOT}}}{N_{\mathrm{Int}}^{\mathrm{TOT}}}+\frac{\delta N_{\mathrm{Inc}}^{\mathrm{TOT}}}{N_{\mathrm{Inc}}^{\mathrm{TOT}}}\right)
$$

where:

$$
\begin{array}{r}
\delta N_{\mathrm{Inc}}^{\mathrm{TOT}}=\sqrt{N_{\mathrm{Inc}}^{\mathrm{TOT}}} \\
\delta N_{\mathrm{Int}}^{\mathrm{TOT}}=\sqrt{N_{\mathrm{Int}}^{\mathrm{TOT}}\left(1-\frac{N_{\mathrm{Int}}^{\mathrm{TOT}}}{N_{\mathrm{Inc}}^{\mathrm{TOT}}}\right)} .
\end{array}
$$

\subsubsection{Treatment of Systematics}

The systematic effects considered in the measurement of the raw cross section result from the uncertainty on the track pitch $(\delta X=0.47 \pm 0.03 \mathrm{~cm}$, shown in 5.5.2) and from the propagation of the uncertainty associate with the measured kinetic energy at each argon slab. As shown in Section 5.6.1, the uncertainty on the kinetic energy 
of a pion candidate at the $\mathrm{j}^{\text {th }}$ slab of argon is given by

$$
\begin{aligned}
\delta K E_{j} & =\sqrt{\delta p_{\text {Beam }}^{2}+\delta E_{\text {Loss }}^{2}+\delta E_{\text {dep FF-j }}^{2}} \\
& =\sqrt{\left(2 \% p_{\text {Beam }}\right)^{2}+(6[\mathrm{MeV}])^{2}+(j-1)^{2}(\sim 0.08[\mathrm{MeV}])^{2}}
\end{aligned}
$$

We propagate this uncertainty by varying the energy measurement $K E_{j}$ at each argon slab. We measure $N_{\text {Inc }}^{\text {TOT }}, N_{\text {Int }}^{\text {TOT }}$ and the cross section in three cases: first assigning the measured $K E_{j}$ at each kinetic energy sampling, then assigning $K E_{j}+$ $\delta K E_{j}$, and finally assigning $K E_{j}-\delta K E_{j}$. The difference between the values obtained using the $K E_{j}$ sampling and the maximum and minimum values in each kinetic energy bin determines the systematic uncertainty.

\subsection{Corrections to the Raw Cross Section}

As described in Section 4.3.3, we need to apply a background correction and an efficiency correction in order to derive the pion cross section from the raw cross section. The cross section is given in Equation 4.9,

$$
\sigma_{T O T}^{\pi^{-}}\left(E_{i}\right)=\frac{1}{n \delta X} \frac{\epsilon^{\mathrm{Inc}}\left(E_{i}\right) C_{\mathrm{Int}}^{\pi M C}\left(E_{i}\right) N_{\mathrm{Int}}^{\mathrm{TOT}}\left(E_{i}\right)}{\epsilon^{\mathrm{Int}}\left(E_{i}\right) C_{\mathrm{Inc}}^{\pi M C}\left(E_{i}\right) N_{\mathrm{Inc}}^{\mathrm{TOT}}\left(E_{i}\right)}
$$

Section 6.2.1 describes the evaluation of pion content in the interacting and inci-

dent histograms, $\left(C_{\mathrm{Int}}^{\pi M C}\left(E_{i}\right)\right.$ and $\left.C_{\mathrm{Inc}}^{\pi M C}\left(E_{i}\right)\right)$ and the propagation to the cross section measurement of the relative systematic uncertainties.

Section 6.2 .2 describes the procedure employed to obtain the efficiency corrections $\epsilon^{\mathrm{Int}}\left(E_{i}\right)$ and $\epsilon^{\mathrm{Inc}}\left(E_{i}\right)$ and the propagation to the cross section measurement of the relative uncertainties. 


\subsubsection{Background subtraction}

We use the procedure described in 5.3 .2 to evaluate the relative pion content in the interacting histogram $C_{\operatorname{Int}}^{\pi M C}\left(E_{i}\right)$ and the relative pion content in the incident $C_{\text {Inc }}^{\pi M C}\left(E_{i}\right)$. We start by evaluating the relative pion content assuming the beamline composition simulated by G4Beamline, whose pion, muon and electron percentages per beam setting are reported again in the first line of Table 6.1. The left side of Figure 6.4 shows the MC estimated relative pion content for the interacting histogram as function of kinetic energy for the 60A runs (top) and 100A runs (bottom). The right side of the same figure shows the $\mathrm{MC}$ estimated relative pion content for the incident histogram as function of kinetic energy for the 60A runs (top) and 100A runs (bottom). In Figure 6.4 the central curves displayed in light blue are obtained using the beamline composition as predicted by G4Beamline: these are the correction curves for the relative pion content applied to data in Equation 4.9 .

So, the question now becomes: how well do we know the beamline composition? In absence of additional data constraints, we take a $100 \%$ systematic uncertainty on the electron content, reported in lines 3 and 4 of Table 6.1. The effect of doubling or halving the electron percentage in the beam on the pion relative content is displayed in red in Figure 6.4. We reserve a slightly different treatment for the muon content. Since G4Beamline tracks only particles which cross all the wire chambers, pion events that decay in flight from $\mathrm{WC} 1$ to $\mathrm{WC} 4$ are not recorded by G4Beamline. Pion decays in the beamline could trigger the beamline detectors in data, if the produced muon propagates forward along the beamline. Thus, we take the G4Beamline prediction for muons as a lower bound in the composition: the effect of doubling the muon content (line 2 in Table 6.1) is shown in blue on Figure 6.4. A future study of data from additional beamline detectors such as the Aerogel Chernkov detectors [11] or the muon range stack (see Section 3.2.4) has the potential of a narrowing the systematics uncertainty coming from the beamline compositon. 
We propagate the uncertainty on the beamline composition as a systematic uncertainty to the cross section by varying the beam composition for all the cases listed in Table 6.1 and evaluating the variation of the obtained data cross sections in each bin. This systematic uncertainty is summed in quadrature with the statistical uncertainty and the systematic uncertainty calculated for the raw cross section.

\subsubsection{Correction for Reconstruction Effects}

The interaction point for a particle in the selected sample for the total hadronic cross section analysis is the last point of its track that lies inside the LArTPC fiducial volume. This definition holds regardless the type of the interaction, i.e. if the TPC track ends within the fiducial volume, its last point will be the interaction point, no matter what the products of the interaction look like; conversely, if the track crosses the boundaries of the fiducial volume, the particle will be considered "through going" and no interaction point will be found. Given this definition, it is evident that we rely on the tracking algorithm to discern where the interaction occurred in the TPC and correctly end the tracking. The tracking algorithm has an intrinsic angle resolution as shown in Section 5.5.1, which limits its efficiency, especially in the case of elastic scattering occurring a low angles. Also, as mentioned in Section 4.3.3, the tracking algorithm can prematurely stop, mis-identifying an interaction point. Thus, we need to apply a correction accounting for mis-reconstruction of the interaction point in order to retrieve the final cross section. This correction is evaluated separately for the interacting and incident histograms bin by bin, namely $\epsilon^{\mathrm{int}}\left(E_{i}\right)$ and $\epsilon^{\mathrm{inc}}\left(E_{i}\right)$, and applied in the cross section formula as shown in Equation 4.9 .

\section{Reconstruction Effects Correction: Procedure}

We describe here the procedure to calculate the mis-reconstruction correction taking the interacting distribution as example and noting that the procedure is identical for 
Background Correction, Interacting

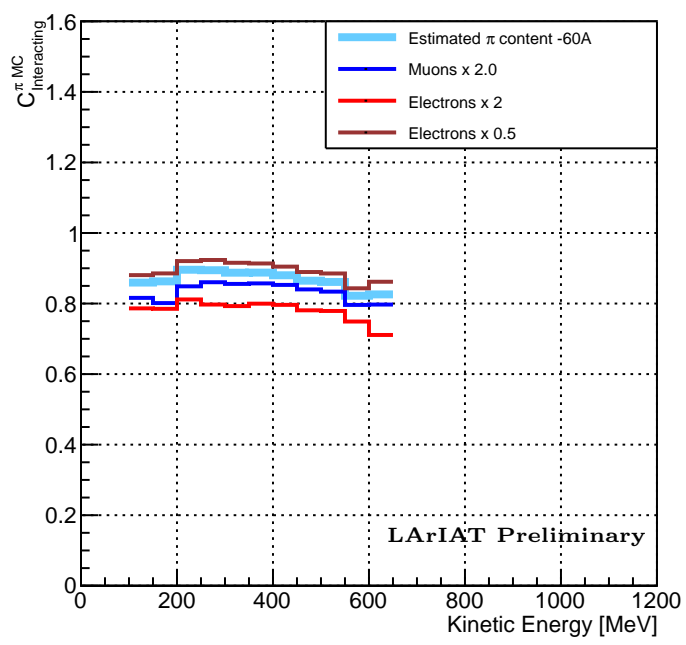

Background Correction, Interacting

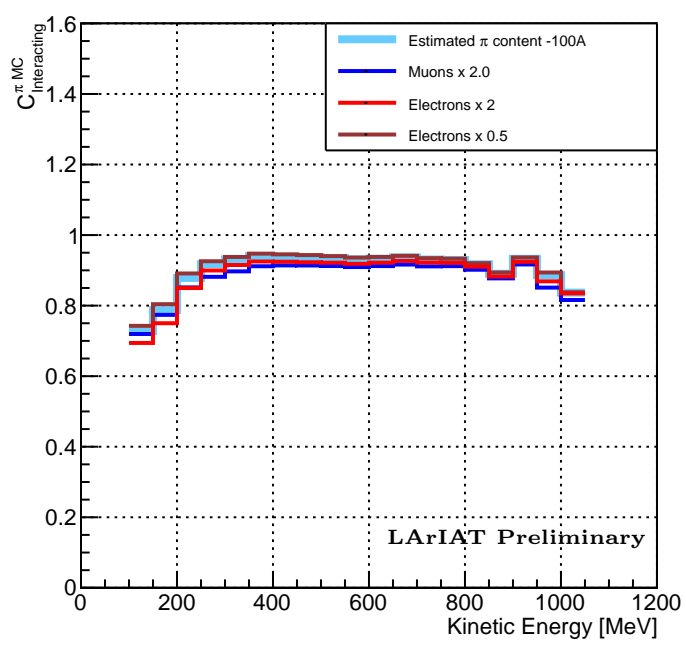

Background Correction, Incident

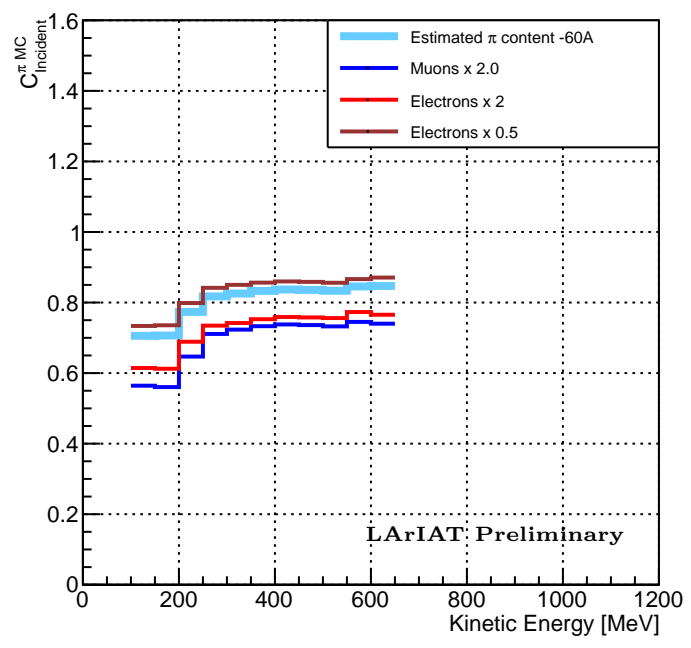

Background Correction, Incident

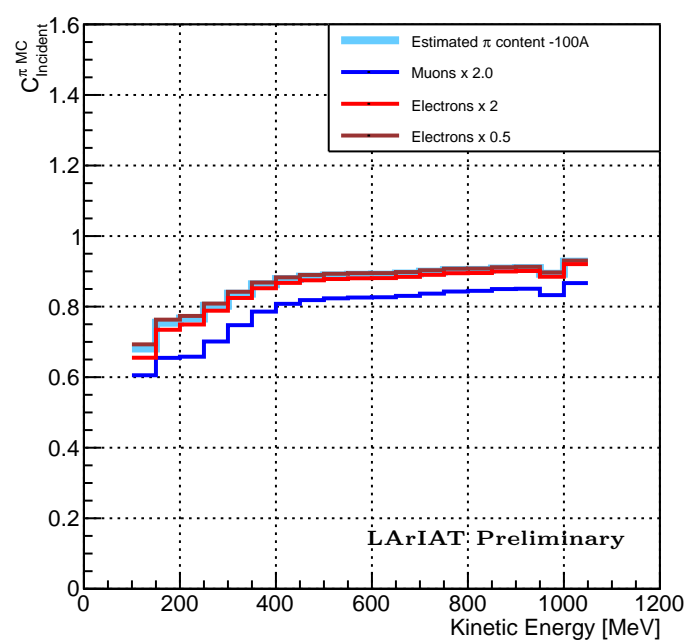

Figure 6.4: Left: $\mathrm{MC}$ estimated relative pion content for interacting histogram a function of kinetic energy for the 60A runs (top) and 100A runs (bottom), predicted background content in azure and muon and electron content variation in blue and red. Right: $\mathrm{MC}$ estimated relative pion content for incident histogram a function of kinetic energy for the 60A runs (top) and 100A (bottom), predicted background content in azure and muon and electron content variation in blue and red 
the incident distribution.

In Section 5.5.1, we estimated the angular resolution for data and $\mathrm{MC}$ to be $\bar{\alpha}_{\text {Data }}=(5.0 \pm 4.5) \mathrm{deg}$ and $\bar{\alpha}_{M C}=(4.5 \pm 3.9) \mathrm{deg}$, respectively. Most interaction angles smaller than the angular resolution will thus be indistinguishable for the reconstruction. Thus, we claim we are able to measure the cross section for interaction angles greater than 5.0 deg. Geant4 simulates interactions at all angles, as shown in figure 6.7. In order to calculate the correction for reconstruction effects, we select events which have an interaction angle greater than a given $\alpha_{\text {res }}$ to construct the true $\mathrm{MC}$ interacting and incident histograms (the denominator of the correction).

We derive the correction $\epsilon^{\mathrm{int}}\left(E_{i}\right)$ on a set of pure pion MC, calculating its value bin by bin as the ratio between the true bin content and the correspondent reconstructed bin content. The true interacting distribution is obtained applying the thin slice method on true $\mathrm{MC}$ energy deposition up to the $\mathrm{MC}$ flagged true interaction point for interaction angles greater than $5^{\circ}$. The reconstructed MC interacting distribution is obtained treating the MC events through the same reconstruction process as data: the interaction point is given by the end of the tracking and its energy is given by the reconstructed calorimetric information. The correction is then applied to in data bin by bin. In formulae, the correction is calculated to be

$$
\epsilon^{\mathrm{Int}}\left(E_{i}\right)=\frac{N_{\text {Interacting }}^{\pi \text { Reco } \mathrm{MC}}\left(E_{i}\right)}{N_{\text {Interacting }}^{\pi \text { True }}\left(E_{i}\right)},
$$

where $N_{\text {Int }}^{\pi}$ True MC $\left(E_{i}\right)$ is the content of the $i$-th bin in the true interacting histogram, and $N_{\mathrm{Int}}^{\pi} \operatorname{Reco\mathrm {MC}}\left(E_{i}\right)$ is the content of the $i$-th bin in the reconstructed interacting histogram. The correction is applied to data as follows

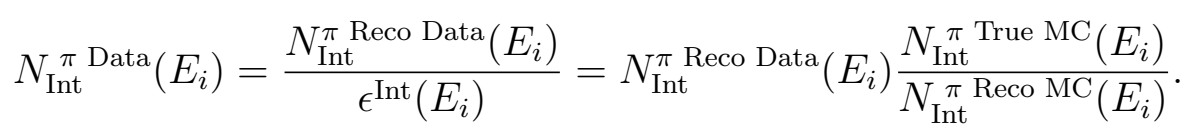


where $N_{\text {Int }}^{\pi}$ Reco Data $\left(E_{i}\right)$ is the background subtracted bin content of the $i$-th bin in for the reconstructed interacting histogram for data, i.e.

$$
N_{\text {Int }}^{\pi \text { Reco Data }}\left(E_{i}\right)=N_{\text {Int }}^{\text {TOT Data }}\left(E_{i}\right)-B_{\text {Int }}^{\text {Data }}\left(E_{i}\right)=C_{\text {Int }}^{\pi \mathrm{MC}}\left(E_{i}\right) N_{\text {Int }}^{\text {TOT Data }}\left(E_{i}\right)
$$

The systematics on this correction is estimated by varying the value of $\alpha_{\text {res }}$ between $0 \mathrm{deg}$ and $5.0 \mathrm{deg}$ and propagating the uncertainty on the cross section.

Figure 6.5 shows $\epsilon^{\operatorname{Int}}\left(E_{i}\right)$ in the left side and $\epsilon^{\mathrm{Inc}}\left(E_{i}\right)$ on the right as a function of the kinetic energy for the 60A runs and their systematic uncertainty. Similarly, Figure 6.6 shows $\epsilon^{\mathrm{Int}}\left(E_{i}\right)$ in the left side and $\epsilon^{\mathrm{Inc}}\left(E_{i}\right)$ on the right as a function of the kinetic energy for the 100A runs and their systematic uncertainty. 


\begin{tabular}{|l|l|l|l|l|l|l|}
\hline & \multicolumn{3}{|c|}{ Magnet Current -60A } & \multicolumn{3}{|c|}{ Magnet Current -100 A } \\
& MC $\pi^{-}$ & MC $\mu^{-}$ & MC $e^{-}$ & MC $\pi^{-}$ & MC $\mu^{-}$ & MC $e^{-}$ \\
\hline Expected Composition & $68.8 \%$ & $4.6 \%$ & $26.6 \%$ & $87.4 \%$ & $3.7 \%$ & $8.9 \%$ \\
Composition $2 \times \mu$ & $64.2 \%$ & $9.2 \%$ & $26.6 \%$ & $83.7 \%$ & $7.4 \%$ & $8.9 \%$ \\
Composition $2 \times e$ & $42.2 \%$ & $4.6 \%$ & $53.2 \%$ & $78.5 \%$ & $3.7 \%$ & $17.8 \%$ \\
Composition $0.5 \times e$ & $82.1 \%$ & $4.6 \%$ & $13.3 \%$ & $91.9 \%$ & $3.7 \%$ & $4.4 \%$ \\
\hline
\end{tabular}

Table 6.1: Beam composition variation for the study of systematics due to beam contamination.
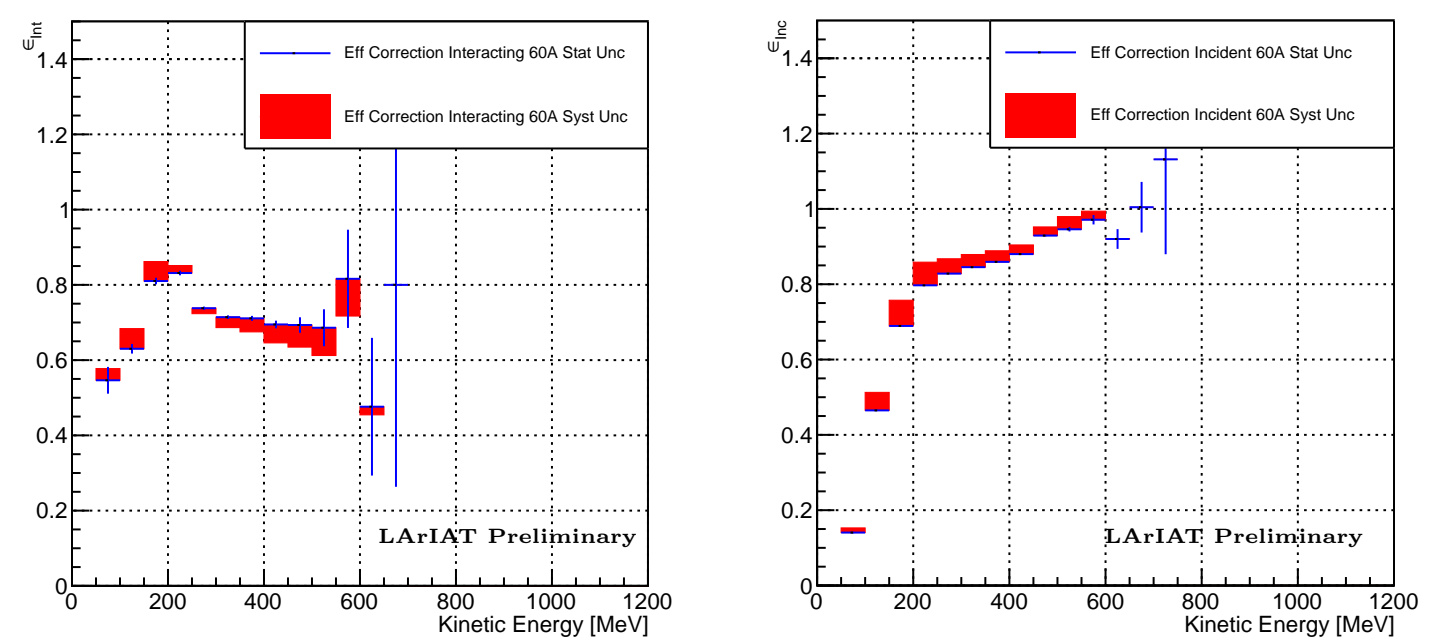

Figure 6.5: Left: Reconstruction effects correction on the 60A interacting histogram, statistical uncertainty in blue, systematic uncertainty in red. Right: Reconstruction effects correction on the $60 \mathrm{~A}$ incident histogram, statistical uncertainty in blue, systematic uncertainty in red. 

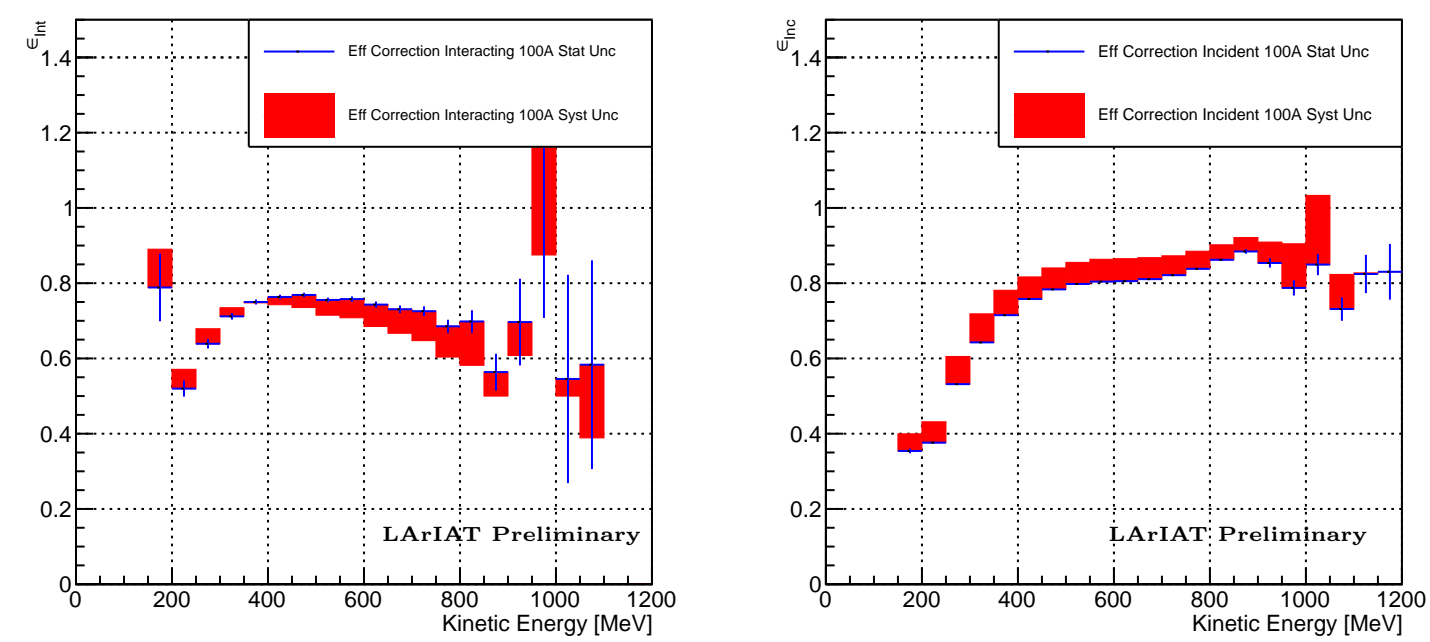

Figure 6.6: Left: Reconstruction effects correction on the 100A interacting histogram, statistical uncertainty in blue, systematic uncertainty in red. Right: Reconstruction effects correction on the $100 \mathrm{~A}$ incident histogram, statistical uncertainty in blue, systematic uncertainty in red.

True Elastic Scattering Angle

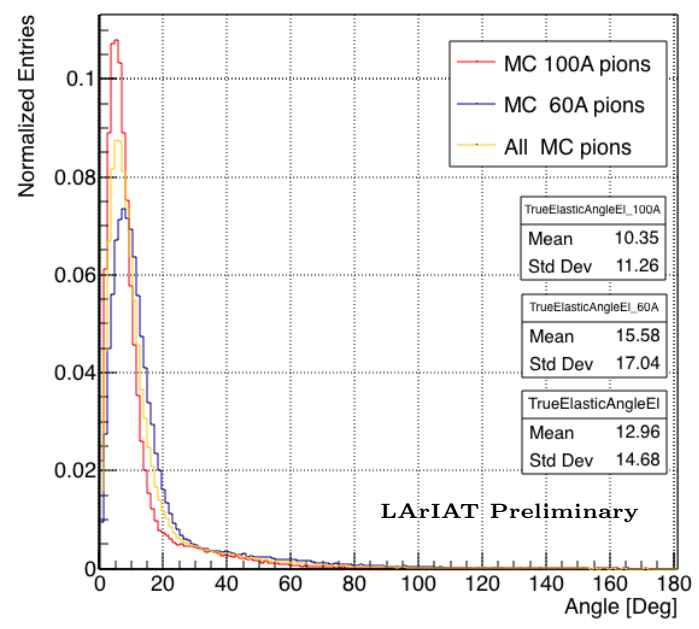

Figure 6.7: Distribution of the true scattering angle for a pion elastic scattering off the argon nucleus as simulated by Geant4. 


\subsection{Results}

Figure 6.8 shows the measurement of the $\left(\pi^{-}-\mathrm{Ar}\right)$ total hadronic cross section,

$$
\sigma_{T O T}^{\pi^{-}}\left(E_{i}\right)=\frac{1}{n \delta X} \frac{N_{\mathrm{Int}}^{\pi^{-}}\left(E_{i}\right)}{N_{\mathrm{Inc}}^{\pi^{-}}\left(E_{i}\right)}=\frac{1}{n \delta X} \frac{\epsilon^{\mathrm{Inc}}\left(E_{i}\right)\left[N_{\mathrm{Int}}^{\mathrm{TOT}}\left(E_{i}\right)-B_{\mathrm{Int}}\left(E_{i}\right)\right]}{\epsilon^{\mathrm{Int}}\left(E_{i}\right)\left[N_{\mathrm{Inc}}^{\mathrm{TOT}}\left(E_{i}\right)-B_{\mathrm{Inc}}\left(E_{i}\right)\right]},
$$

for scattering angles greater than $5^{\circ}$, as the result of the background subtraction and reconstruction effects correction to the raw cross section. The top left plot is the measurement obtained on the 60A data, statistical and systematic uncertainty shown in red. The top right plot is the measurement obtained on the $100 \mathrm{~A}$ data, statistical uncertainty and systematic uncertainty shown in blue. The bottom plot shows the two measurements overlaid. In all three plot, the FTFP_BERT Geant4 prediction for the total hadronic cross section for angle scattering greater than $5^{\circ}$ is displayed in green.

The systematic uncertainty on the cross section is the sum in quadrature of the statistical uncertainty, the systematic uncertainty calculated for the raw cross section, the systematic uncertainty related to the beam composition and the systematic uncertainty related to the reconstruction effects correction.

The cross section measurements in the two datasets agree within the systematic uncertainty, even if the cross section measured in the $60 \mathrm{~A}$ data is lower than the one measured in the $100 \mathrm{~A}$ data in all overlapping bins except for the $200-250 \mathrm{MeV}$ bin. With the exception of the highest energy bins, the systematic uncertainties are the dominant uncertainties for both the $60 \mathrm{~A}$ and $100 \mathrm{~A}$ datasets.

The top panel of Figure 6.9 shows the cross section obtained combining the two datasets. To combine the datasets, we merge the corrected interacting histogram for the $60 \mathrm{~A}$ data with the corrected interacting histogram for the $100 \mathrm{~A}$ data; we apply the same merging with the incident histograms. We then calculate the cross section 
central point, statistical uncertainty and systematic uncertainty relative to the kinetic energy using the merged interacting and incident histograms. The systematic uncertainty relative to the corrections is calculated as the sum in quadrature of the original systematic uncertainties on the cross section measurements on the two separated data sets. Again, with the exception of the highest energy bins (energy greater than 800 $\mathrm{MeV}$ ), the systematic uncertainties are the dominant uncertainties; the lion's share of the systematic uncertainty results from our uncertainty on the beam composition, which we plan to constrain with different beamline data in a future iteration of the analysis.

The bottom panel of Figure 6.9 shows the relative deviation between the measured cross section and the FTFP_BERT Geant4 predicted cross section. A couple of considerations are in order here. On one hand, we note the general agreement between the measured and predicted cross sections. We expected the pion cross section in argon to be well modeled in Geant4, given the vast set of measurements available for pion interaction on different targets - even if no previous data is available on argon. Thus, the data-MC agreement is expected and confirms the validity of the experimental methodology used. On the other hand, this agreement is not perfect. As a matter of fact, its imperfections are rather suggestive: we see a slight shift of the delta resonance at higher energies and a small shape difference, with a steeper drop between the delta region and the flatter part at energies greater than $400 \mathrm{MeV}$. As we showed in Figure 1.4, the shape of the delta resonance changes as a function of the nuclear target; thus it is plausible that the Geant4 model under estimates the cross section for argon in the delta resonance region and overestimates it in the higher energy region. Since we see room for improvement of the systematic uncertainties, we look forward to seeing if this difference in shape will be confirmed by further analyses, leading to a re-tuning of the model. 

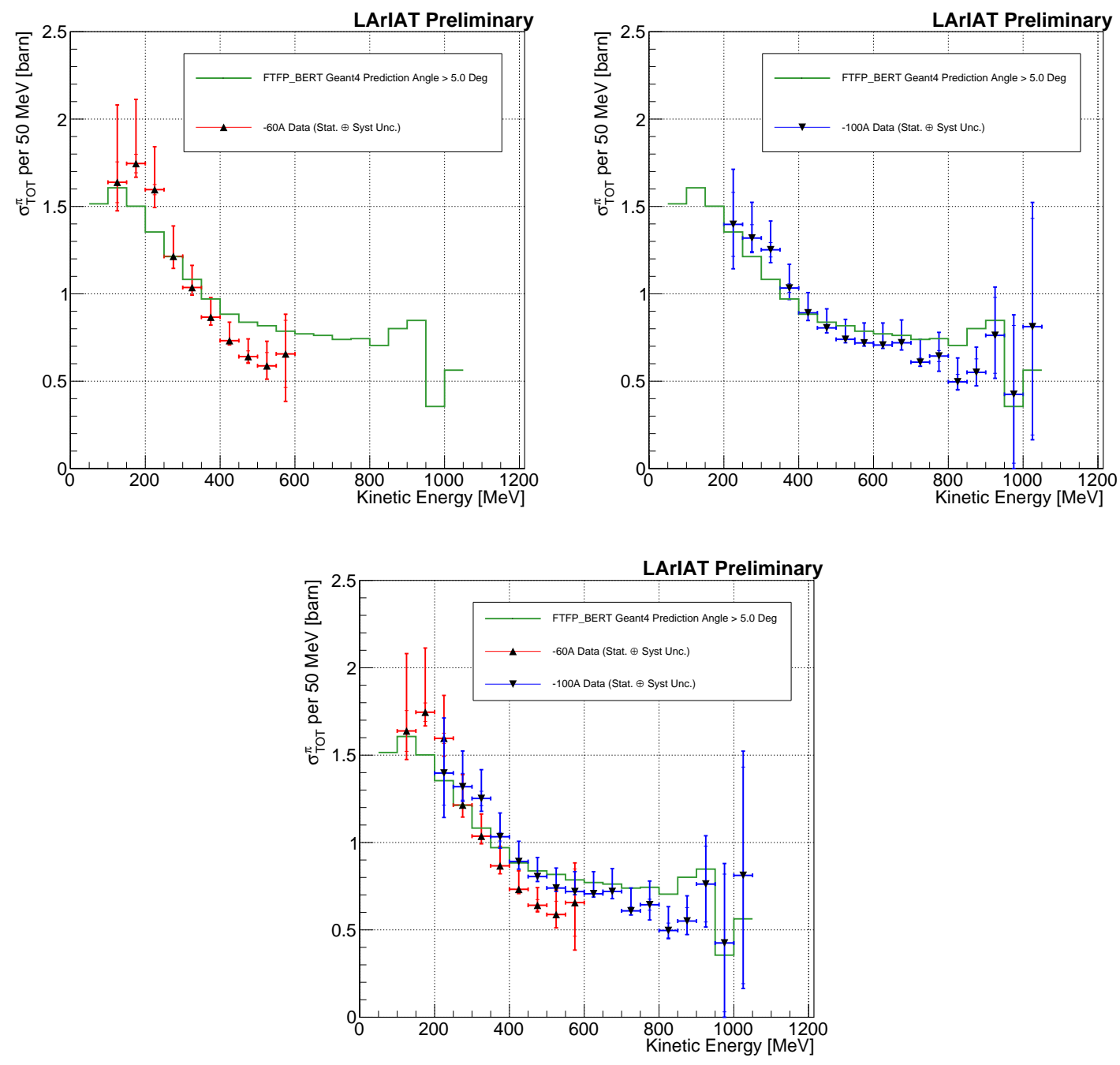

Figure 6.8: Top Left: $\left(\pi^{-}-\mathrm{Ar}\right)$ total hadronic cross section for scattering angles greater than $5^{\circ}$ measured in the $60 \mathrm{~A}$ sample, statistical uncertainty and systematic uncertainty in red. The FTFP_BERT Geant4 prediction for the total hadronic cross section for angle scattering greater than $5^{\circ}$ is displayed in green.

Top Right: $\left(\pi^{-}-\mathrm{Ar}\right)$ total hadronic cross section for scattering angles greater than $5^{\circ}$ measured in the $100 \mathrm{~A}$ sample, statistical uncertainty and systematic uncertainty in blue. The FTFP_BERT Geant4 prediction for the total hadronic cross section for angle scattering greater than $5^{\circ}$ is displayed in green.

Bottom: ( $\left.\pi^{-}-\mathrm{Ar}\right)$ total hadronic cross section measurements in the $60 \mathrm{~A}$ and $100 \mathrm{~A}$ samples overlaid with the FTFP_BERT Geant4 prediction (green). 


\section{LArIAT Preliminary}
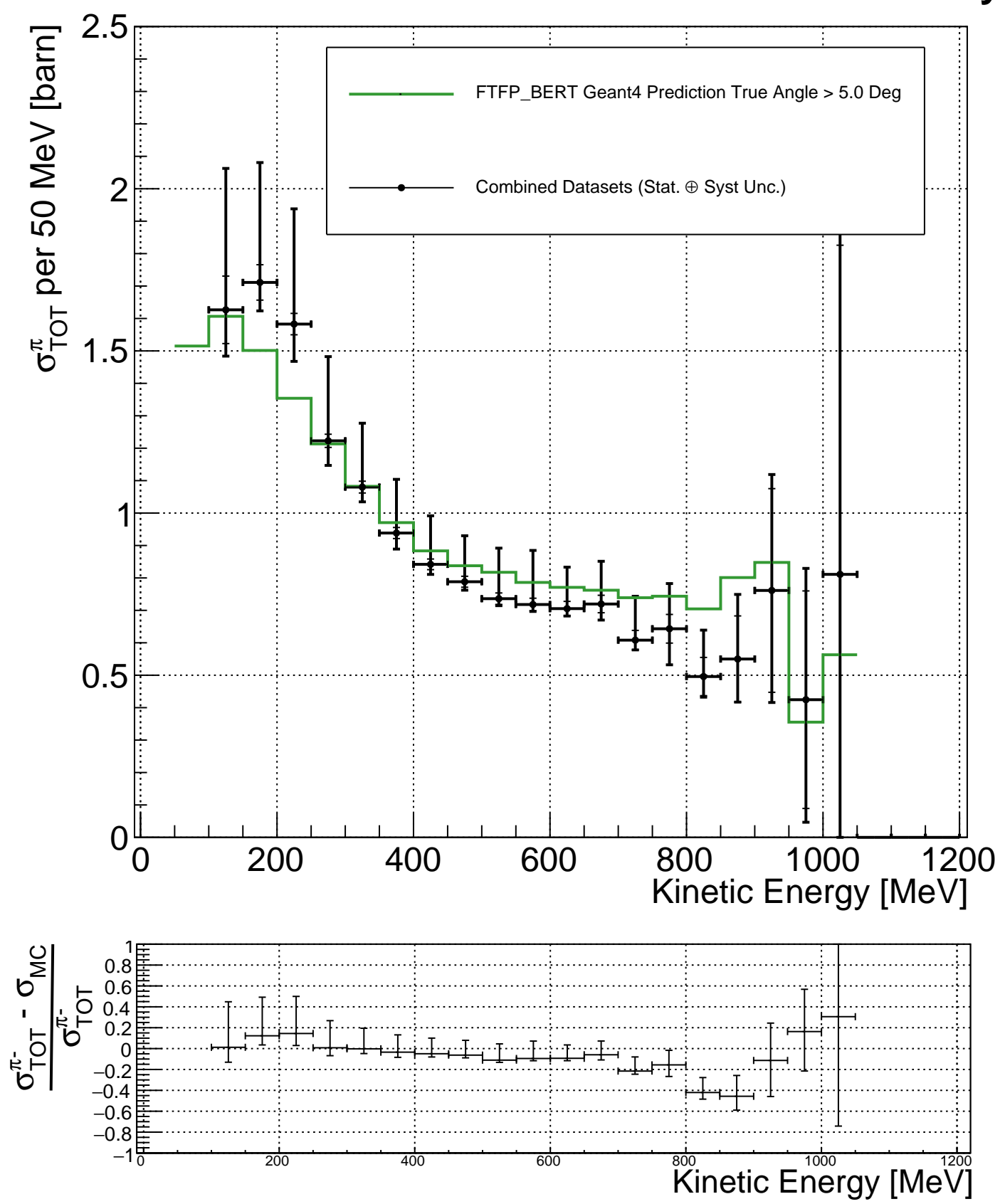

Figure 6.9: Top: $\left(\pi^{-}-\mathrm{Ar}\right)$ total hadronic cross section for scattering angles greater than $5^{\circ}$ measured in the combined sample, statistical uncertainty and systematic uncertainty in black. The Geant4 prediction for the total hadronic cross section for angle scattering greater than $5^{\circ}$ is displayed in green. Bottom: Relative difference between the measured cross section and the Geant4 prediction. 


\title{
Chapter 7
}

\section{Positive Kaon Cross Section}

\section{Measurement}

\author{
"Beat-up little seagull, on a marble stair \\ Tryin' to find the ocean, lookin' everywhere." \\ - Nina Simone, 1978 -
}

In this chapter, we show the results of the thin slice method to measure the $\left(K^{+}-\mathrm{Ar}\right)$ total hadronic cross section. In Section 7.1, we start by measuring the raw cross section. In Section 7.2, we apply a statistical subtraction of the background contributions based on simulation and a correction for reconstruction effects. The final results are presented in Section 7.3 .

\subsection{Raw Cross Section}

We measure the raw $\left(K^{+}-\mathrm{Ar}\right)$ total hadronic cross section as a function of the kinetic energy in the combined $+60 \mathrm{~A}$ and $+100 \mathrm{~A}$ dataset. 
Similar to the pion case, the raw cross section is given by the Equation 4.4

$$
\sigma_{T O T}\left(E_{i}\right)=\frac{1}{n \delta X} \frac{N_{\mathrm{Int}}^{\mathrm{TOT}}\left(E_{i}\right)}{N_{\mathrm{Inc}}^{\mathrm{TOT}}\left(E_{i}\right)},
$$

where $N_{\text {Int }}^{\mathrm{TOT}}$ is the measured number of particles interacting at kinetic energy $E_{i}$, $N_{\text {Inc }}^{\text {TOT }}$ is the measured number of particles incident on an argon slice at kinetic energy $E_{i}, n$ is the density of the target centers and $\delta X$ is the thickness of the argon slice. The density of the target centers and the slab thickness are $n=0.021 \cdot 10^{24} \mathrm{~cm}^{-3}$ and $\delta X=0.47 \pm 0.03 \mathrm{~cm}$, respectively.

As in the case of pions, kaons might decay or interact between WC4 and the TPC front face. Some of the interaction products may be wrongly matched to the WC track, forming the "secondary" particle's background in the kaon sample. We estimate the effect of the contamination of secondaries through the DDMC kaon sample. Figure 7.1 shows the distribution of $N_{\text {Int }}^{\mathrm{TOT}}$ as a function of the kinetic energy. The data central points are represented by black dots, the statistical uncertainty is shown in black, while the systematic uncertainty is shown in red. Data is displayed over the $N_{\text {Int }}^{\text {TOT }}$ distribution obtained with a DDMC sample of kaons shot from WC4. The contribution from the simulated kaons which interact hadronically is shown in pink, the contributions from kaon decay is shown in orange and the one from secondaries in red. The simulated kaon's and secondaries' contributions are stacked; the sum of their integrals is normalized to the integral of the data.

Figure 7.2 shows the distribution of $N_{\mathrm{Inc}}^{\mathrm{TOT}}$. Data is displayed over MC. For the $N_{\text {Inc }}^{\text {TOT }}$ distribution we do not make a distinction between kaons that decay or interact hadronically because any kaon contributes to the flux of incident particles at given kinetic energy independently from its final interaction. The same normalization procedure is used for both the interacting and incident histograms.

Figure 7.3 shows the raw cross section, statistical uncertainty in black and system- 
atic uncertainty in red. The raw data cross section is overlaid to the reconstructed cross section for the MC sample, displayed in azure. We calculate the statistical uncertainty for the interacting, incident and cross section distributions in a similar fashion to the pion case as described in Section 6.1.1.

As in the pion case, the systematic effects considered in the measurement of the raw cross section results from the uncertainty on the pitch $(\delta X=0.47 \pm 0.03 \mathrm{~cm})$ and from the propagation of the uncertainty associate with the measurement of the kinetic energy at each argon slab. For kaons, the uncertainty on the kinetic energy of a candidate at the $\mathrm{j}^{\text {th }}$ slab of argon is given by

$$
\begin{aligned}
\delta K E_{j} & =\sqrt{\delta p_{\text {Beam }}^{2}+\delta E_{\text {Loss }}^{2}+\delta E_{\text {dep FF-j }}^{2}} \\
& =\sqrt{\left(2 \% p_{\text {Beam }}\right)^{2}+(7[\mathrm{MeV}])^{2}+(j-1)^{2}(\sim 0.18[\mathrm{MeV}])^{2}}
\end{aligned}
$$

We propagate this uncertainty by varying the energy measurement $K E_{j}$ at each argon slab. We measure $N_{\text {Inc }}^{\text {TOT }}, N_{\text {Int }}^{\text {TOT }}$ and the cross section in three cases: first assigning the measured $K E_{j}$ at each kinetic energy sampling, then assigning $K E_{j}+$ $\delta K E_{j}$, and finally assigning $K E_{j}-\delta K E_{j}$. The difference between the values obtained using the $K E_{j}$ sampling and the maximum and minimum values in each kinetic energy bin determines the systematic uncertainty.

\subsection{Corrections to the Raw Cross Section}

As described in Section 4.3.3, we need to apply a background correction and a correction for reconstruction effects in order to derive the kaon cross section from the raw cross section. The cross section is given in Equation 4.9 , 


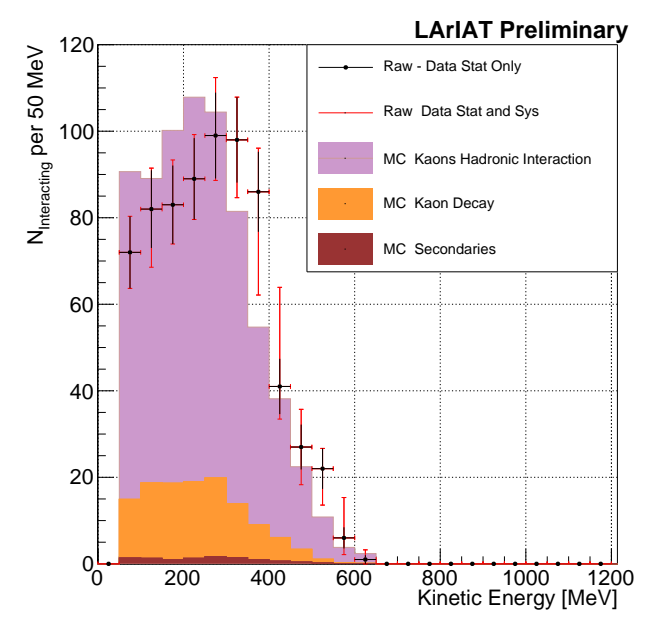

Figure 7.1: Raw number of interacting kaon candidates as a function of the reconstructed kinetic energy. The statistical uncertainties are shown in black, the systematic uncertainties in red.

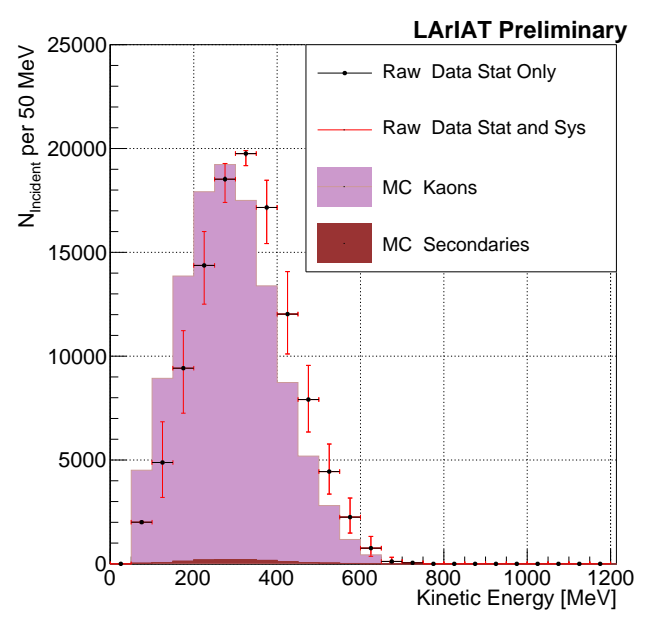

Figure 7.2: Raw number of incident kaon candidates as a function of the reconstructed kinetic energy. The statistical uncertainty is shown in black, the systematic uncertainties in red.

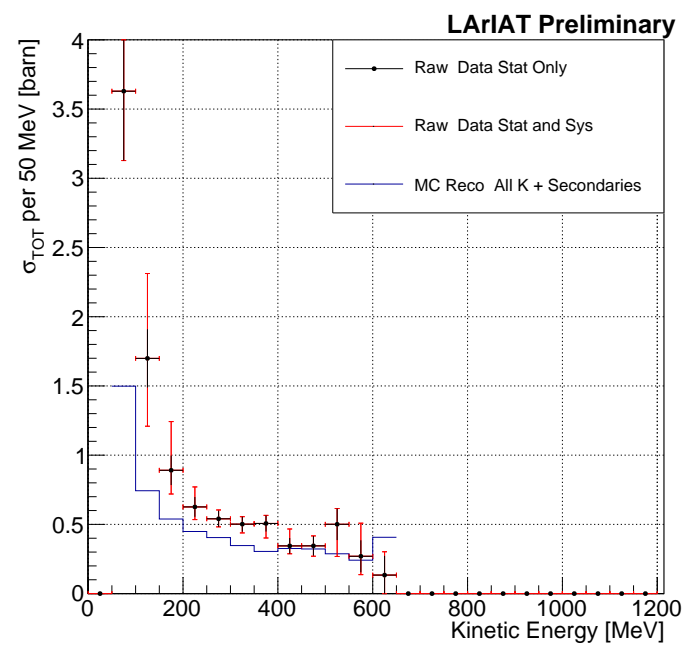

Figure 7.3: Raw $\left(K^{+}-\mathrm{Ar}\right)$ total hadronic cross section. The statistical uncertainty is shown in black, the systematic uncertainties in red. The raw cross section obtained with a MC sample of kaons is shown in azure. For the MC cross section, we include the contributions from secondaries. 


$$
\sigma_{T O T}^{K^{+}}\left(E_{i}\right)=\frac{1}{n \delta X} \frac{\epsilon^{\mathrm{Inc}}\left(E_{i}\right) C_{\mathrm{Int}}^{K M C}\left(E_{i}\right) N_{\mathrm{Int}}^{\mathrm{TOT}}\left(E_{i}\right)}{\epsilon^{\mathrm{Int}}\left(E_{i}\right) C_{\mathrm{Inc}}^{K M C}\left(E_{i}\right) N_{\mathrm{Inc}}^{\mathrm{TOT}}\left(E_{i}\right)} .
$$

Currently, the only background considered for the kaon hadronic cross section comes from the presence of secondaries and decay interaction points. A further development of the analysis will need to account for the presence of a small proton contamination (estimated to be $\sim 5 \%$ in Section 5.2.1). Figure 7.4 shows the relative kaon content suitable for the hadronic cross section for the interacting and incident histograms.

As described in 6.2 .2 for the pion case, we derive the correction on a set of pure kaon $\mathrm{MC}$, calculating its value bin by bin as the ratio between the true bin content and the correspondent reconstructed bin content. The correction is then applied to the relevant bin in data. The correction for recontruction effects is evaluated separately for the interacting and incident histograms, namely $\epsilon^{\mathrm{int}}\left(E_{i}\right)$ and $\epsilon^{\mathrm{inc}}\left(E_{i}\right)$, and propagated to the cross section as shown in Equation 4.9 .

In Section 5.5.1, we estimated the angular resolution for data and $\mathrm{MC}$ to be $\bar{\alpha}_{\text {Data }}=(4.3 \pm 3.7) \mathrm{deg}$ and $\bar{\alpha}_{M C}=(4.4 \pm 3.6) \mathrm{deg}$, respectively. Most interaction angles smaller than the angular resolution will thus be indistinguishable for the reconstruction. Thus, we claim we are able to measure the cross section for interaction angles greater than 4.5 deg. Geant4 simulates interactions at all angles: in order to calculate the correction for reconstruction effects, we select events which have an interaction angle greater than a $\alpha_{\text {res }}$ to construct the true interacting and incident histograms (the denominator of the correction). The systematics on this correction is estimated by varying the value of $\alpha_{\text {res }}$ between 0 deg and $4.5 \mathrm{deg}$ and propagating the uncertainty on the cross section.

Figure 7.5 shows $\epsilon^{\operatorname{Int}}\left(E_{i}\right)$ in the left side and $\epsilon^{\operatorname{Inc}}\left(E_{i}\right)$ on the right as a function of the kinetic energy for the kaon sample and their systematic uncertainty. 

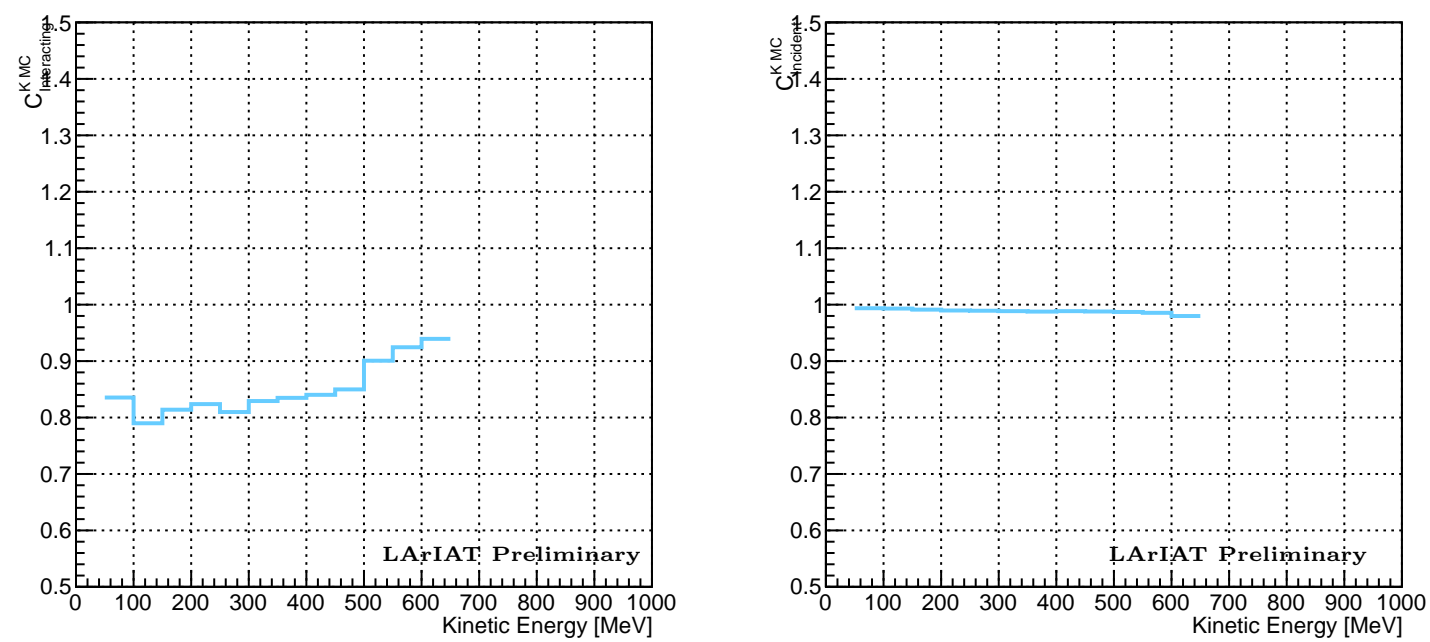

Figure 7.4: Left: MC estimated relative kaon content for kaons interacting hadronically as function of kinetic energy. Right: $\mathrm{MC}$ estimated relative kaon content for incident histogram a function of kinetic energy.
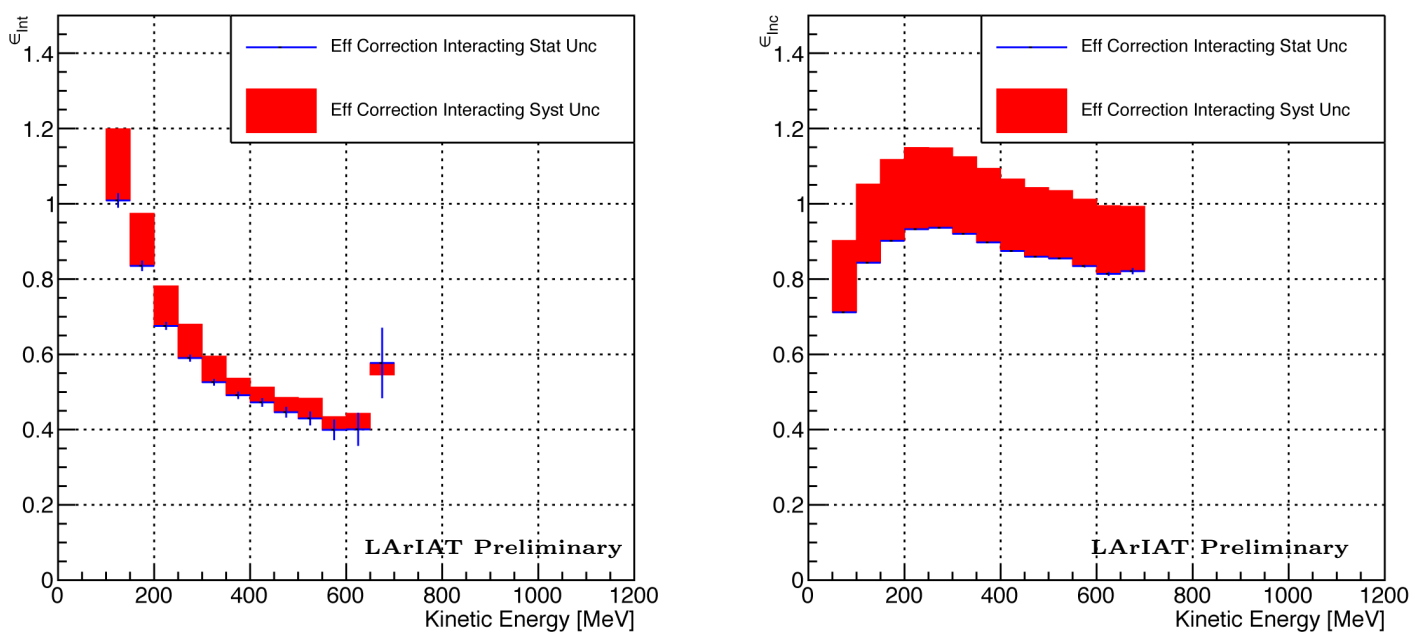

Figure 7.5: Left: Reconstruction effects correction on the interacting histogram, statistical uncertainty in blue, systematic uncertainty in red. Right: Reconstruction effects correction on the incident histogram, statistical uncertainty in blue, systematic uncertainty in red. 


\subsection{Results}

Figure 7.6 show the measurement of the $\left(K^{+}-\mathrm{Ar}\right)$ total hadronic cross section,

$$
\sigma_{T O T}^{K^{+}}\left(E_{i}\right)=\frac{1}{n \delta X} \frac{N_{\mathrm{Int}}^{K^{+}}\left(E_{i}\right)}{N_{\mathrm{Inc}}^{K^{+}}\left(E_{i}\right)}=\frac{1}{n \delta X} \frac{\epsilon^{\mathrm{Inc}}\left(E_{i}\right)\left[N_{\mathrm{Int}}^{\mathrm{TOT}}\left(E_{i}\right)-B_{\mathrm{Int}}\left(E_{i}\right)\right]}{\epsilon^{\mathrm{Int}}\left(E_{i}\right)\left[N_{\mathrm{Inc}}^{\mathrm{TOT}}\left(E_{i}\right)-B_{\mathrm{Inc}}\left(E_{i}\right)\right]}
$$

for scattering angles greater than $5^{\circ}$, as the result of the background subtraction and reconstruction effects correction to the raw cross section. The plot shows the measurement obtained on the full dataset, statistical and systematic uncertainty in black. The Geant4 prediction for the total hadronic cross section for angle scattering greater than $5^{\circ}$ is displayed in green.

The systematic uncertainty on the cross section is the sum in quadrature of the statistical uncertainty, the systematic uncertainty calculated for the raw cross section and the systematic uncertainty related to the reconstruction effects correction. In every bin, the statistical uncertainty is the dominant uncertainty; a future iteration

of the analysis using the "High Yield" sample, which is expected to boost the statistics by a factor of 3 , will reduce the statistical uncertainty.

The measured cross section is mostly in tension with the predicted cross section; in particular it is significantly higher - on the order of $20 \%-30 \%$ higher - in the bins lower than $400 \mathrm{MeV}$. Such difference is unlikely to be accounted for the small proton contamination expected in the data sample. It is worth noticing that the $100 \mathrm{MeV}$ to $400 \mathrm{MeV}$ energy range is the region of interest for the expected kaons coming from a proton decay event. With this result, we will inform a tuning of the Geant4 model for kaon cross section. 


\section{LArIAT Preliminary}
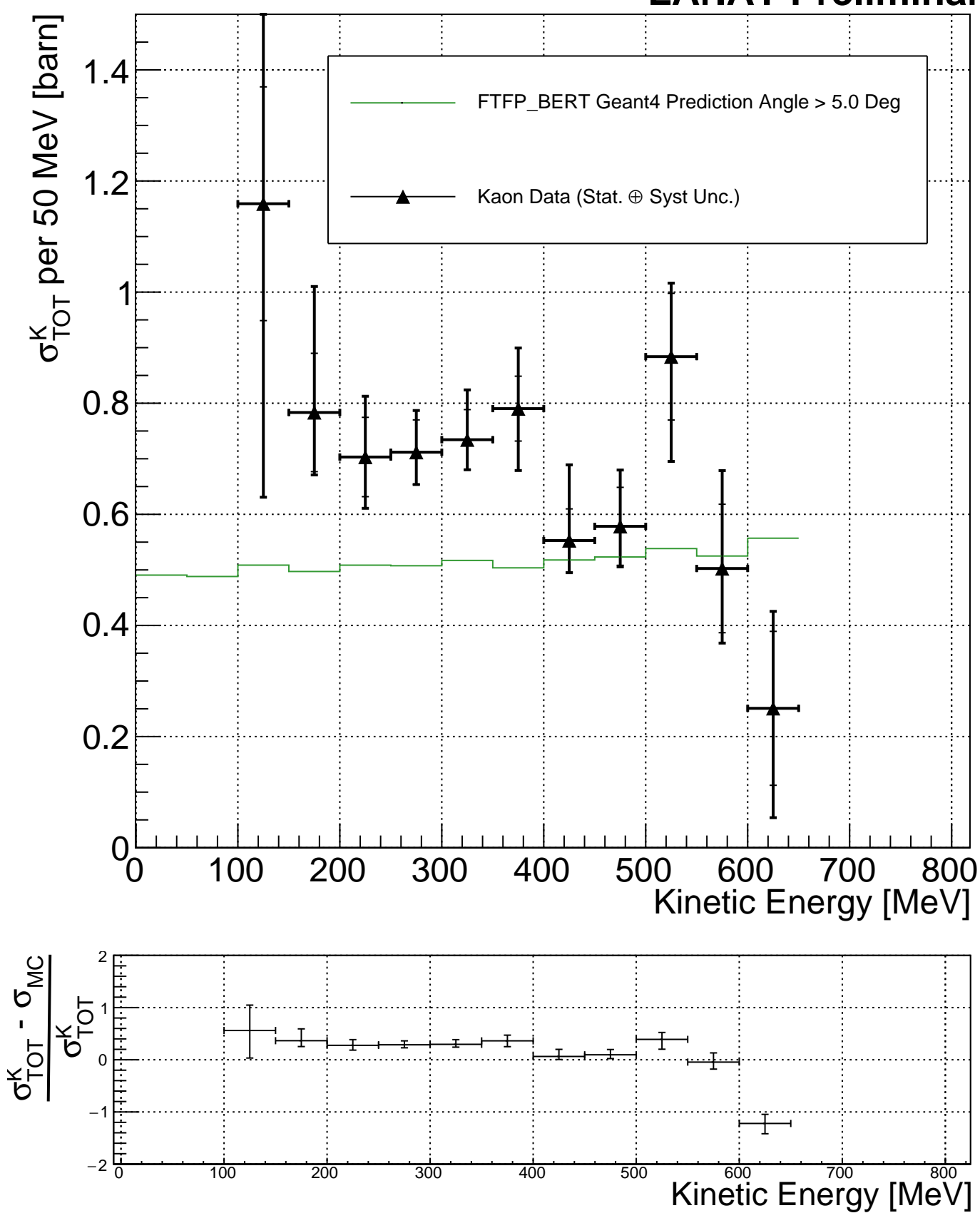

Figure 7.6: Top: $\left(K^{+}-\mathrm{Ar}\right)$ total hadronic cross section for scattering angles greater than $5^{\circ}$ measured in the combined $+60 \mathrm{~A}$ and $+100 \mathrm{~A}$ data samples, statistical uncertainty and systematic uncertainty in black. The Geant4 prediction for the total hadronic cross section for angle scattering greater than $5^{\circ}$ is displayed in green. Bottom: Relative deviation between the measured cross section and the Geant4 prediction. 


\section{Chapter 8}

\section{Conclusions}

In the era of neutrino precision physics, of huge liquid argon detectors and of massive amount of information from LArTPCs, a renewed interest in an ancient measurement arises: the measurement of hadronic interactions with matter. With this work, we presented the first ever $\left(\pi^{-}-\mathrm{Ar}\right)$ and $\left(K^{+}-\mathrm{Ar}\right)$ total hadronic cross section measurements as a function of the hadron kinetic energy. Both the analyses follow a similar workflow and they rely on beam line detector information as well as both calorimetry and tracking in the LArTPC.

For the $\left(\pi^{-}-\mathrm{Ar}\right)$ total hadronic cross section measurement, we start by identifying pion beamline candidates through a series of selections on the beamline and TPC information which maximize the number of pions over other particle species (muons and electrons). We use the LArIAT beamline MC to estimate the beam composition of the selected beamline candidates and we propagate them to the LArTPC constructing a properly weighted sample with the DDMC. We apply the thin slice method on the pion candidates and obtain the raw cross section. From the simulated sample, we obtain two corrections accounting for the beamline background contamination and for reconstruction effects. Finally, we apply the corrections to data and measure the cross section. 
In order to measure $\left(K^{+}-\mathrm{Ar}\right)$ total hadronic argon cross sections, we follow a similar procedure, i.e. we apply the thin slice method on kaon candidates identified in the beamline and obtain the raw cross section. We then apply a MC derived background correction and a correction for detector effects to measure the cross section. The background correction accounts for the presence of secondary particles in both the interacting and incident histograms and for the presence of decay events in the interacting plot.

The final results for the $\left(\pi^{-}-\mathrm{Ar}\right)$ and $\left(K^{+}-\mathrm{Ar}\right)$ total hadronic cross section are shown side by side in the top part of Figure 8.1. The bottom part of the same figure shows the relative deviation from the cross section predicted by Geant4. In the pion case, the measured cross section mostly agrees with the Geant4 prediction. A hint of a difference in shape is present: Geant4 seems to underestimate the cross section in the delta resonance region and overestimate it for energies greater than $400 \mathrm{MeV}$. Since the $\left(\pi^{-}\right.$-Ar) has never been measured before on argon and since previous measurements on different nuclei show that the shape of the delta resonance peak varies as a function of the target nucleus, this hint is an interesting feature of the measurement which LArIAT will explore with a future development of the analysis. The outcome of this measurement will ultimately enable to quantify and reduce the systematic associated with the hadronic interaction models in neutrinoargon interactions.

In the kaon case, the measured cross section is mostly higher than the Geant4 prediction. Contrary to the pion case, previous measurements of kaon interaction on any nuclei are scarce; thus, we do not expect the Geant4 prediction to be as solid in the kaon case as it is in the pion case - as illustrated by Figure 1.13 for kaon cross section on carbon. The nice agreement between the measured cross section and the prediction of the pion case gives us confidence in the methodology, thus confidence in the kaon measurement. With the kaon cross section measurement, we hope to inform 
the next generation of Geant4 model tuning, thus providing a more reliable tool in the simulation for proton decay searches.

The $\left(\pi^{-}-\mathrm{Ar}\right)$ and $\left(K^{+}-\mathrm{Ar}\right)$ total hadronic cross section analyses are the first physics analyses developed by the LArIAT experiment and will serve as a basis for the future cross section measurements of pion and kaon cross sections in the exclusive channels.
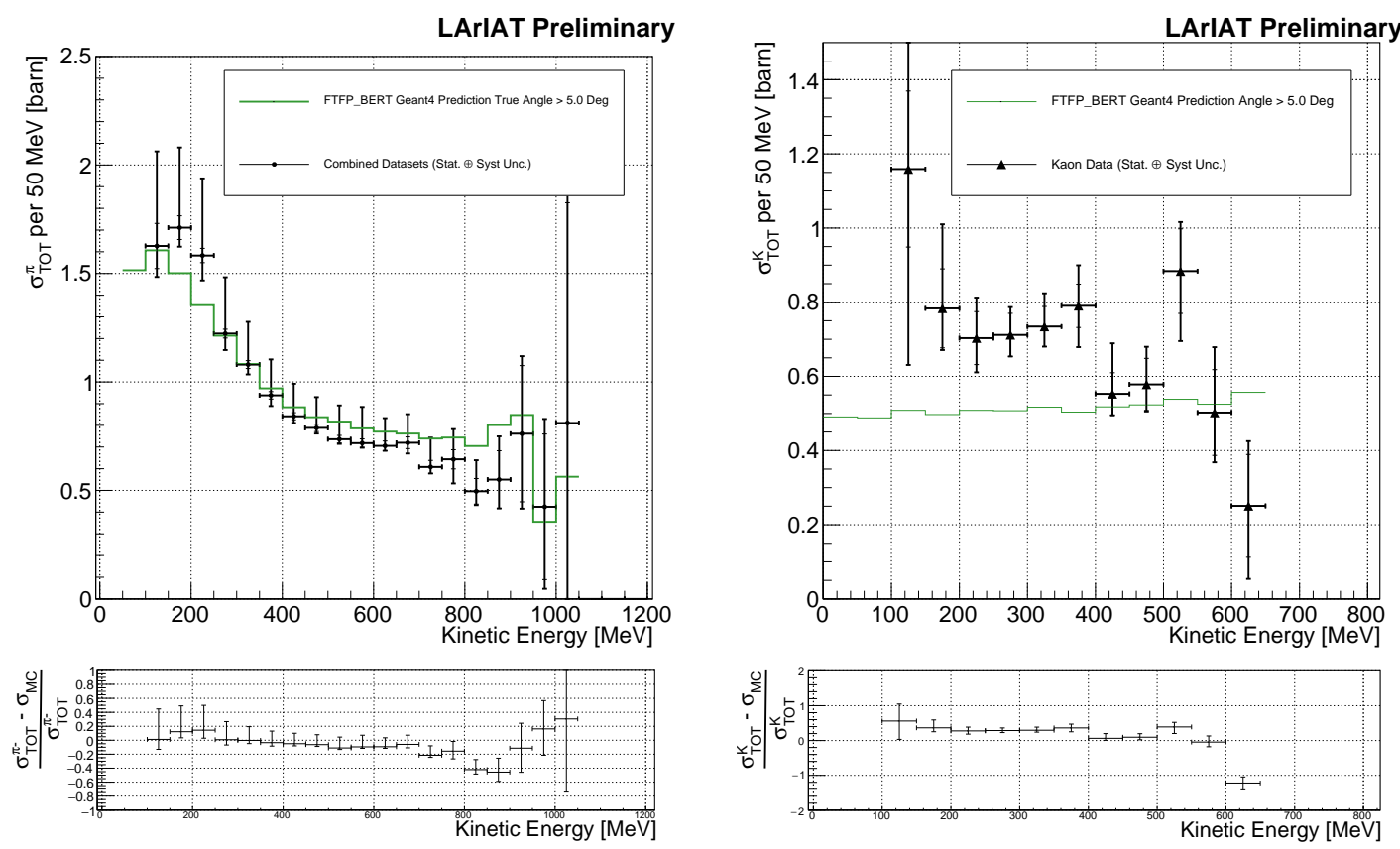

Figure 8.1: Left: $\left(\pi^{-}-\mathrm{Ar}\right)$ total hadronic cross section measurement for scattering angles greater than $5^{\circ}$ measured in the combined sample overlaid with the Geant4 prediction (green). Right: $\left(K^{+}-\mathrm{Ar}\right)$ total hadronic cross section for scattering angles greater than $5^{\circ}$ measured. The respective Geant4 prediction for the total hadronic cross section for angle scattering greater than $5^{\circ}$ is displayed in green. 


\section{Appendix A}

\section{Measurement of LArIAT Electric}

\section{Field}

The electric field of a LArTPC in the drift volume is a fundamental quantity for the proper functionality of this technology, as it affects almost every reconstructed quantity such as the position of hits or their collected charge. Given its importance, we calculate the electric field for LArIAT with a single line diagram from our HV circuit and we cross check the obtained value with a measurement relying only on TPC data.

Before getting into the details of the measurement procedures, it is important to clarify the relationship between the quantities in play. The electric field $\left(E_{\text {field }}\right)$ and the drift velocity $\left(v_{d r i f t}\right)$ are related as follows

$$
v_{\text {drift }}=\mu\left(E_{\text {field }}, T\right) E_{\text {field }},
$$

where $\mu$ is the electron mobility, which depends on the electric field and on the temperature $(\mathrm{T})$. The empirical formula for this dependency is described in 121 and shown in Figure A.1 for several argon temperatures.

The relationship between the drift time $\left(t_{d r i f t}\right)$ and the drift velocity is trivially 


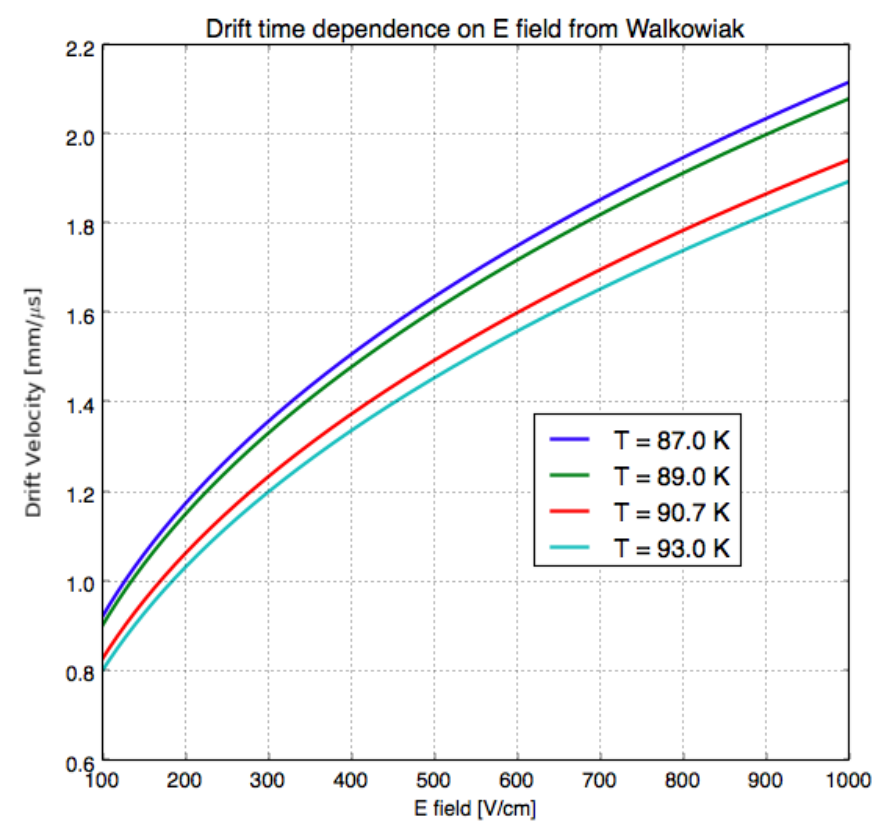

Figure A.1: Drift velocity dependence on electric field for several temperatures. The slope of the line at any one point represents the electron mobility for that given temperature and electric field.

Table A.1: Electric field and drift velocities in LArIAT smaller drift volumes

\begin{tabular}{|l|l|l|}
\hline & Shield-Induction & Induction-Collection \\
\hline $\mathrm{E}_{\text {field }}$ & $700.63 \mathrm{~V} / \mathrm{cm}$ & $892.5 \mathrm{~V} / \mathrm{cm}$ \\
\hline $\mathrm{v}_{\text {drift }}$ & $1.73 \mathrm{~mm} / \mu \mathrm{s}$ & $1.90 \mathrm{~mm} / \mu \mathrm{s}$ \\
\hline $\mathrm{t}_{\text {drift }}$ & $2.31 \mu \mathrm{s}$ & $2.11 \mu \mathrm{s}$ \\
\hline
\end{tabular}

given by

$$
t_{d r i f t}=\Delta x / v_{d r i f t}
$$

where $\Delta x$ is the distance between the edges of the drift region. Table A.1 reports the values of the electric field, drift velocity, and drift times for the TPC's small drift volumes.

With these basic parameters established, we can now move on to calculating the electric field in the main drift region (between the cathode and the shield plane). 


\section{Single line diagram method}

The electric field strength in the LArIAT main drift volume can be determined knowing the voltage applied to the cathode, the voltage applied at the shield plane, and the distance between them. We assume the distance between the cathode and the shield plane to be $470 \mathrm{~mm}$ and any length contraction due to the liquid argon is negligibly small $(\sim 2 \mathrm{~mm})$.

The voltage applied to the cathode can be calculated using Ohm's law and the single line diagram shown in Figure A.2. A set of two of filter pots for emergency power dissipation are positioned between the Glassman power supply and the cathode, one at each end of the feeder cable, each with an internal resistance of $40 \mathrm{M} \Omega$.

Given the TPC resistor chain, the total TPC impedance is $6 \mathrm{G} \Omega$. Since the total resistance on the circuit is driven by the TPC impedance, we expect the resulting current to be

$$
I=V_{P S} / R_{t o t}=-23.5 \mathrm{kV} / 6 \mathrm{G} \Omega \sim 4 \mu \mathrm{A},
$$

which we measure with the Glassman power supply, shown in Figure A.3.

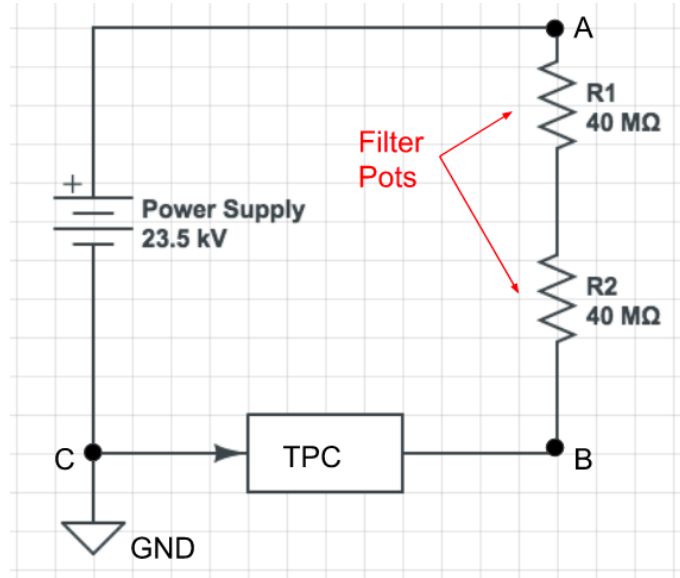

Figure A.2: LArIAT HV simple scheme.

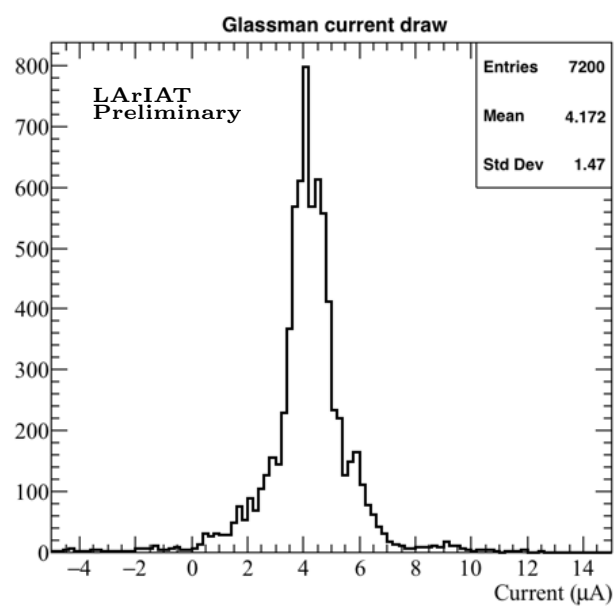

Figure A.3: Current reading from the Glassman between May 25th and May 30th, 2016 (typical Run-II conditions). 
Using this current, the voltage at the cathode is calculated as

$$
V_{B C}=V_{P S}-\left(I \times R_{e q}\right)=-23.5 \mathrm{kV}+(0.00417 \mathrm{~mA} \times 80 \mathrm{M} \Omega)=-23.17 \mathrm{kV},
$$

where $I$ is the current and $R_{e q}$ is the equivalent resistor representing the two filter pots. The electric field is then calculated to be

$$
E_{\text {field }}=\frac{V_{B C}-V_{\text {shield }}}{\Delta x}=486.54 \mathrm{~V} / \mathrm{cm}
$$

\section{E field using cathode-anode piercing tracks}

We devised an independent method to measure the drift time (and consequently drift velocity and electric field) using TPC cathode to anode piercing tracks. We use this method as a cross check to the single line method. The basic idea is simple:

1. Select cosmic ray events with only 1 reconstructed track

2. Reduce the events to the ones containing tracks that cross both anode and cathode

3. Identify the first and last hit of the track

4. Measure the time difference between these two hits $(\Delta t)$.

This method works under the assumptions that the time it takes for a cosmic particle to cross the chamber $(\sim \mathrm{ns})$ is small compared to the charge drift time $(\sim$ hundreds of $\mu \mathrm{s})$.

We choose cosmic events to allow for a high number of anode to cathode piercing tracks (ACP tracks), rejecting beam events where the particles travel almost perpendicularly to drift direction. We select events with only one reconstructed track to maximize the chance of selecting a single crossing muon (no michel electron). We utilize ACP tracks because their hits span the full drift length of the TPC, see Figure 
A.4. allowing us to define where the first and last hit of the tracks are located in space regardless of our assumption of the electric field.

One of the main features of this method is that it doesn't rely on the measurement of the trigger time. Since $\Delta t$ is the time difference between the first and last hit of a track and we assume the charge started drifting at the same time for both hits, the measurement of the absolute beginning of drift time $t_{0}$ is unnecessary. We boost the presence of ACP tracks in the cosmic sample by imposing the following requirements on tracks:

- vertical position (Y) of first and last hits within $\pm 18 \mathrm{~cm}$ from TPC center (avoid Top-Bottom tracks)

- horizontal position (Z) of first and last hits within 2 and $86 \mathrm{~cm}$ from TPC front face (avoid through going tracks)

- track length greater than $48 \mathrm{~cm}$ (more likely to be crossing)

- angle from the drift direction (phi in figure A.5) smaller than 50 deg (more reliable tracking)

- angle from the beam direction (theta in figure A.5) greater than 50 deg (more reliable tracking)

Tracks passing all these selection requirements are used for the $\Delta t$ calculation.

For each track passing our selection, we loop through the associated hits to retrieve the timing information. The analysis is performed separately on hits on the collection plane and induction plane, but leads to consistent results. As an example of the time difference, figures A.6 and A.7 represent the difference in time between the last and first hit of the selected tracks for Run-II Positive Polarity sample on the collection and induction plane respectively. We fit with a Gaussian to the peak of the $\Delta t$ distributions to extract the mean drift time and the uncertainty associated with it. 
The long tail at low $\Delta t$ represents contamination of non-ACP tracks in the track selection. We apply the same procedure to Run-I and Run-II, positive and negative polarity alike.

To convert $\Delta t$ recorded for the hits on the induction plane to the drift time we employ the formula

$$
t_{d r i f t}=\Delta t-t_{S-I}
$$

where $t_{d r i f t}$ is the time the charge takes to drift in the main volume between the cathode and the shield plane and $t_{S-I}$ is the time it takes for the charge to drift from the shield plane to the induction plane. In Table A.1 we calculated the drift velocity in the S-I region, thus we can calculate $t_{S-I}$ as

$$
t_{S-I}=\frac{l_{S-I}}{v_{S-I}}=\frac{4 m m}{1.73 m m / \mu s}
$$

where $l_{S-I}$ is the distance between the shield and induction plane and $v_{S-I}$ is the drift velocity in the same region. A completely analogous procedure is followed for the hits on the collection plane, taking into account the time the charge spent in drifting from shield to induction as well as between the induction and collection plane The value for $\Delta t_{d r i f t}$, the calculated drift velocity $\left(v_{d r i f t}\right)$, and corresponding drift electric field for the various run periods is given in Table A.2 and are consistent with the electric field value calculated with the single line diagram method. 
Delta $\mathbf{t}_{\text {drift }}$, drift $\mathbf{v}$ and $\mathbf{E}$ field with ACP tracks

\begin{tabular}{|l|c|c|c|}
\hline \hline Data Period & $\Delta t_{\text {Drift }}[\mu s]$ & $v_{\text {Drift }}[\mathrm{mm} / \mu \mathrm{s}]$ & $E_{\text {field }}[\mathrm{V} / \mathrm{cm}]$ \\
\hline RunI Positive Polarity Induction & $311.1 \pm 2.4$ & $1.51 \pm 0.01$ & $486.6 \pm 21$ \\
\hline RunI Positive Polarity Collection & $310.9 \pm 2.6$ & $1.51 \pm 0.01$ & $487.2 \pm 21$ \\
\hline RunII Positive Polarity Induction & $315.7 \pm 2.8$ & $1.49 \pm 0.01$ & $467.9 \pm 21$ \\
\hline RunII Positive Polarity Collection & $315.7 \pm 2.7$ & $1.49 \pm 0.01$ & $467.9 \pm 21$ \\
\hline RunII Negative Polarity Induction & $315.9 \pm 2.6$ & $1.49 \pm 0.01$ & $467.1 \pm 21$ \\
\hline RunII Negative Polarity Collection & $315.1 \pm 2.8$ & $1.49 \pm 0.01$ & $470.3 \pm 21$ \\
\hline \hline Average Values & 314.1 & $1.50 \pm 0.01$ & $474.3 \pm 21$ \\
\hline
\end{tabular}

Table A.2: $\Delta t$ for the different data samples used for the Anode-Cathode Piercing tracks study.

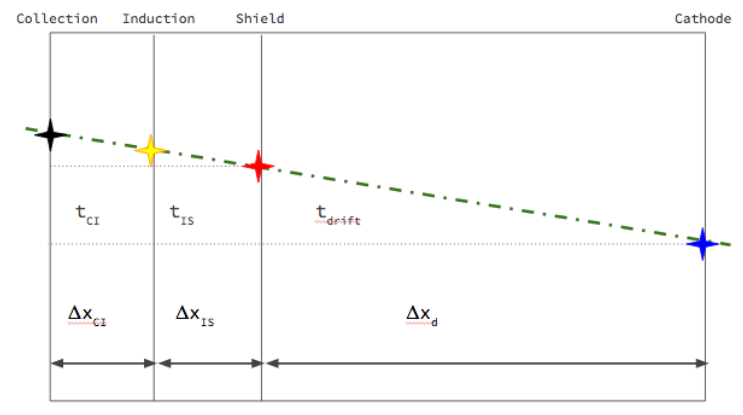

Figure A.4: Pictorial representation of the YX view of the TPC. The distance within the anode planes and between the shield plane and the cathode is purposely out of proportion to illustrate the time difference between hits on collection and induction. An ACP track is shown as an example.

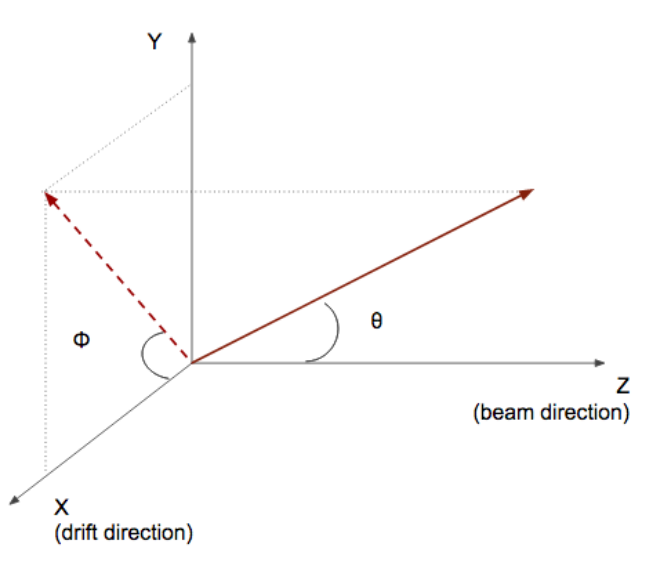

Figure A.5: Angle definition in the context of LArIAT coordinate system. 


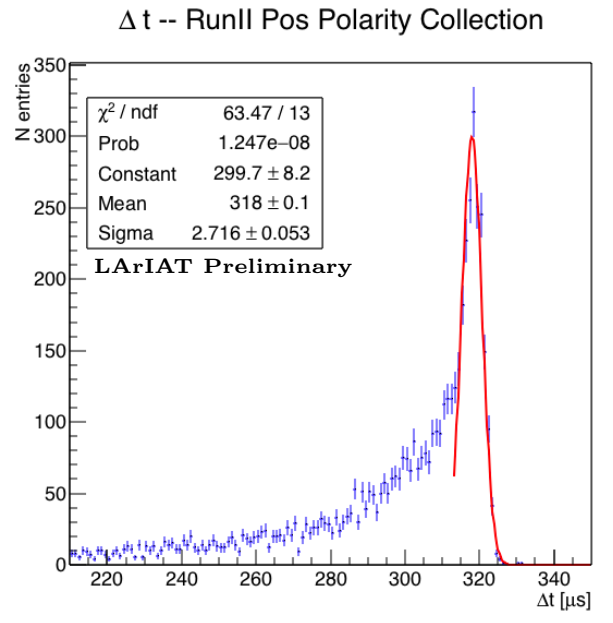

Figure A.6: Collection plane $\Delta \mathrm{t}$ fit for Run II positive polarity ACP data selected tracks.

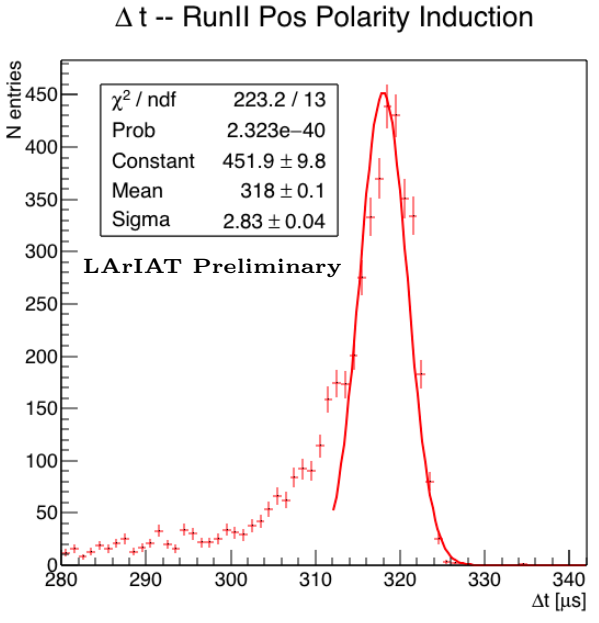

Figure A.7: Induction plane $\Delta \mathrm{t}$ fit for Run II positive polarity ACP data selected tracks. 


\section{Appendix B}

\section{Additional Tracking Studies for LArIAT Cross Section Analyses}

In this section, we describe two studies. The first is a justification of the selection criteria for the beamline handshake with the TPC information. We perform this study to boost the correct identification of the particles in the TPC associated with the beamline information, while maintaining sufficient statistics for the cross section measurement. The second study is an optimization of the tracking algorithm, with the scope of maximizing the identification of the hadronic interaction point inside the TPC. These two studies are related, since the optimization of the tracking is performed on TPC tracks which have been matched to the wire chamber track; in turn, the tracking algorithm for TPC tracks determines the number of reconstructed tracks in each event used to try the matching with the wire chamber track. Starting with a sensible tracking reconstruction, we perform the WC2TPC matching optimization first, then the tracking optimization. The WC2TPC match purity and efficiency are then calculated again with the optimized tracking. 


\section{B.1 Study of WC to TPC Match}

Scope of this study is assessing the performances of the WC2TPC match on Monte Carlo (see Section 4.2) and decide the selection values we will use on data. A word of caution is necessary here. With this study, we want to minimize pathologies associated with the presence of the primary hadron itself, e.g. the incorrect association between the beamline hadron and its decay products inside the TPC. Assessing the contamination from pile-un! albeit related, is beyond the scope of this study.

In MC, we are able to define a correct WC2TPC match using the Geant4 truth information. We are thus able to count how many times the WC tracks is associated with the wrong TPC reconstructed track.

We define a correct match if the all following conditions are met:

- the length of the true primary Geant4 track in the TPC is greater than $2 \mathrm{~cm}$,

- the length of the reconstructed track length is greater than $2 \mathrm{~cm}$,

- the $\mathrm{Z}$ position of the first reconstructed point is within $2 \mathrm{~cm}$ from the TPC front face

- the distance between the reconstructed track and the true entering point is the minimum compared with all the other reconstructed tracks.

In order to count the wrong matches, we consider all the reconstructed tracks whose $\mathrm{Z}$ position of the first reconstructed point lies within $2 \mathrm{~cm}$ from the TPC front face. Events with true length in TPC $<2 \mathrm{~cm}$ are included. Since hadrons are shot $100 \mathrm{~cm}$ upstream from the TPC front face, the following two scenarios are possible from a truth standpoint:

$[\mathrm{Ta}]$ the primary hadron decays or interact strongly before getting to the TPC,

1. We remind the reader that the DDMC is a single particle Monte Carlo, where the beam pile up is not simulated. 
$[\mathrm{Tb}]$ the primary hadron enters the TPC.

As described in Section 4.2, we define a WC2TPC match according to the relative position of the WC and TPC track parametrized with $\Delta R$ and the angle between them, parametrized with $\alpha$. Once we choose the selection values $r_{T}$ and $\alpha_{T}$ to determine a reconstructed $\mathrm{WC} 2 \mathrm{TPC}$ match, the following five scenarios are possible in the truth to reconstruction interplay :

1) only the correct track is matched

2) only one wrong track is matched

3) the correct track and one (or more) wrong tracks are matched

4) multiple wrong tracks matched.

5) no reconstructed tracks are matched

Since we keep only events with one and only one match, we discard cases 3 ), 4) and 5) from the events used in the cross section measurement. For each set of $r_{T}$ and $\alpha_{T}$ selection value, we define purity and efficiency of the selection as follows:

$$
\begin{gathered}
\text { Efficiency }=\frac{\text { Number of events correctly matched }}{\text { Number of events with primary in TPC }}, \\
\text { Purity }=\frac{\text { Number of events correctly matched }}{\text { Total number of matched events }} .
\end{gathered}
$$

Figure B.1 shows the efficiency (left) and purity (right) for WC2TPC match as a function of the radius, $r_{T}$, and angle, $\alpha_{T}$, selection value. It is apparent how both efficiency and purity are fairly flat as a function of the radius selection value at a given angle. This is not surprising. Since we are studying a single particle gun Monte Carlo sample, the wrong matches can occur only for mis-tracking of the primary or 
for association with decay products; decay products will tend to be produced at large angles compared to the primary, but could be fairly close to the in $x$ and $y$ projection of the primary. The radius cut would play a key role in removing pile up events.

For LArIAT cross section measurements, we generally prefer purity over efficiency, since a sample of particles of a pure species will lead to a better measurement. Obviously, purity should be balanced with a sensible efficiency to avoid rejecting the whole sample.

We choose $\left(\alpha_{T}, r_{T}\right)=(8 \mathrm{deg}, 4 \mathrm{~cm})$ and get a $\mathrm{MC} 85 \%$ efficiency and $98 \%$ purity for the kaon sample and a MC 95\% efficiency and 90\% purity for the pion sample.
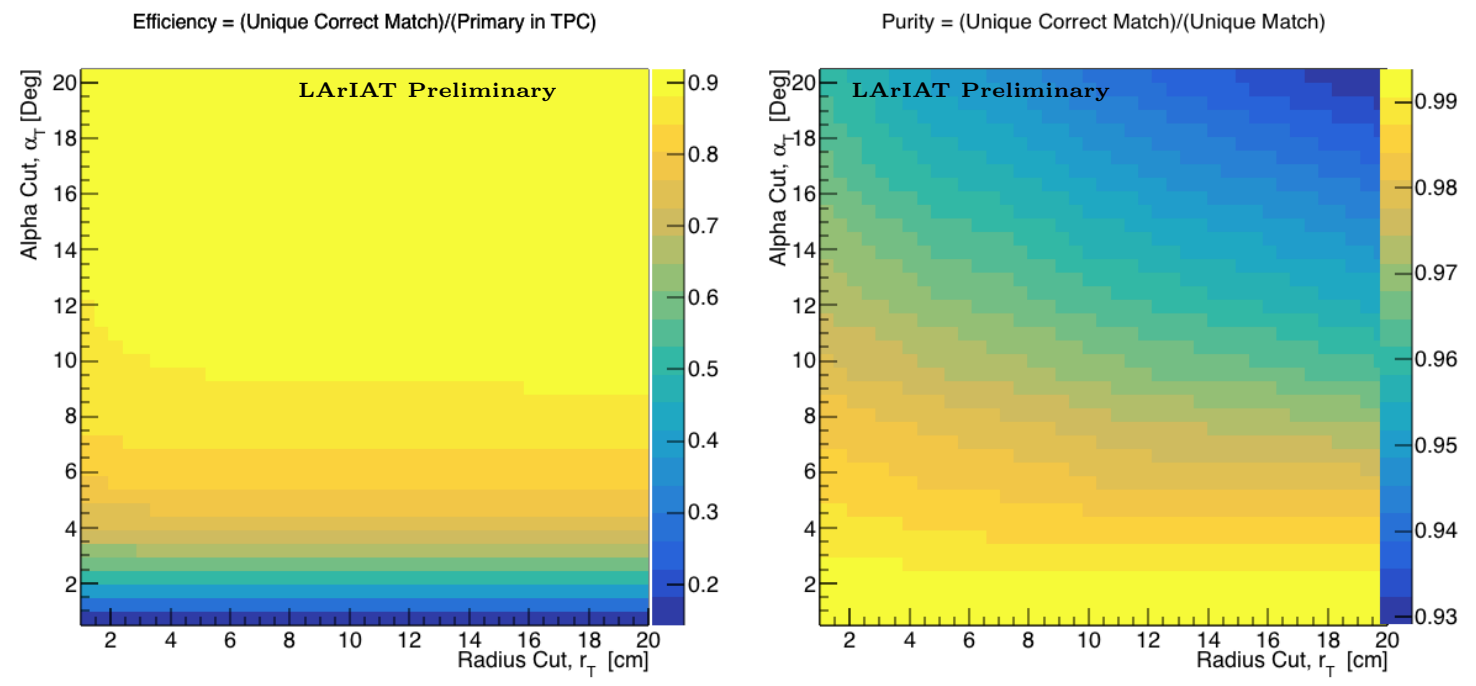

Figure B.1: Efficiency (left) and purity (right) for WC2TPC match as a function of the radius and angle selections for the kaon sample.

\section{B.2 Tracking Optimization}

We perform an optimization of the clustering algorithm (see Section 2.1.5) with the scope of maximizing the efficiency of finding the interaction point for the total hadronic cross section measurements. We define as the interaction point the most downstream point of a WC2TPC matched TPC tracks within the TPC fiducial vol- 
ume. Since all the WC2TPC tracks are by definition beam particles, tracks travel from upstream to downstream in the TPC; thus, identifying the interaction point means to stop the tracking correctly.

TrajCluster is the package used to cluster hits in LArIAT; this package counts more than 20 tunable parameters. A standard method to develop clustering algorithms and checking their performances is to "hand scan", which means recognizing the effect of parameters tuning by looking at a series of data event displays. Albeit we recognize the importance of hand scanning as a great diagnosis tool, we developed a fully automated optimization package which compares $\mathrm{MC}$ reconstructed information to MC truth.

We start by defining a figure of merit in order to discern what makes a parameter configuration better than an other. We chose the percentage of events whose reconstructed and true length differ less than $2 \mathrm{~cm}$. In order to identify the true interaction point, no selection is performed on the scattering angle: even hadronic interaction flagged by Geant 4 with very shallow angles $\left(i 5^{\circ}\right)$ are kept to perform the optimization. We then identify the parameters in TrajCluster that are most important to correctly stop the tracking and an appropriate range of values for each of them. We chose to optimize the parameters that leverage on the angle between consecutive groups of hits, the number of hits use in the cluster fit and the average hit charge to stop the tracking. We define a configuration space with all possible combination of values for the chosen parameters and we perform reconstruction one combination at a time: the combination with the highest figure of merit determines the optimized tracking reconstruction.

We chose construct the combination space using a total of 5 parameters, 3 values each and two iterations of the method (for a total of 486 combinations). We run the combinations on a sample of 100000 pion events. After the optimization, the most upstream point of the tracking is correctly identified $99.5 \%$ of the times, the most 
downstream point is correctly identified $62.5 \%$ of the times, the tracking stops short about $15 \%$ of the times and misses the interaction point $22.5 \%$ of the times. Hand scanning confirmed that the missed interaction points happen in the vast majority of cases for very shallow angles, as shown in the event display in Figure B.2, or in the case of angles visible only in one projection plane. We also noticed that the premature stopping of the tracks is often related to the presence of delta rays parallel to the track. We see room of improvement, such as the delta ray removal and a forced track breaking in case of a kink present in a single plane, for a future analysis.

The procedure behind this optimization package is virtually applicable to any LArSoft module where it is possible to define figure of merit.

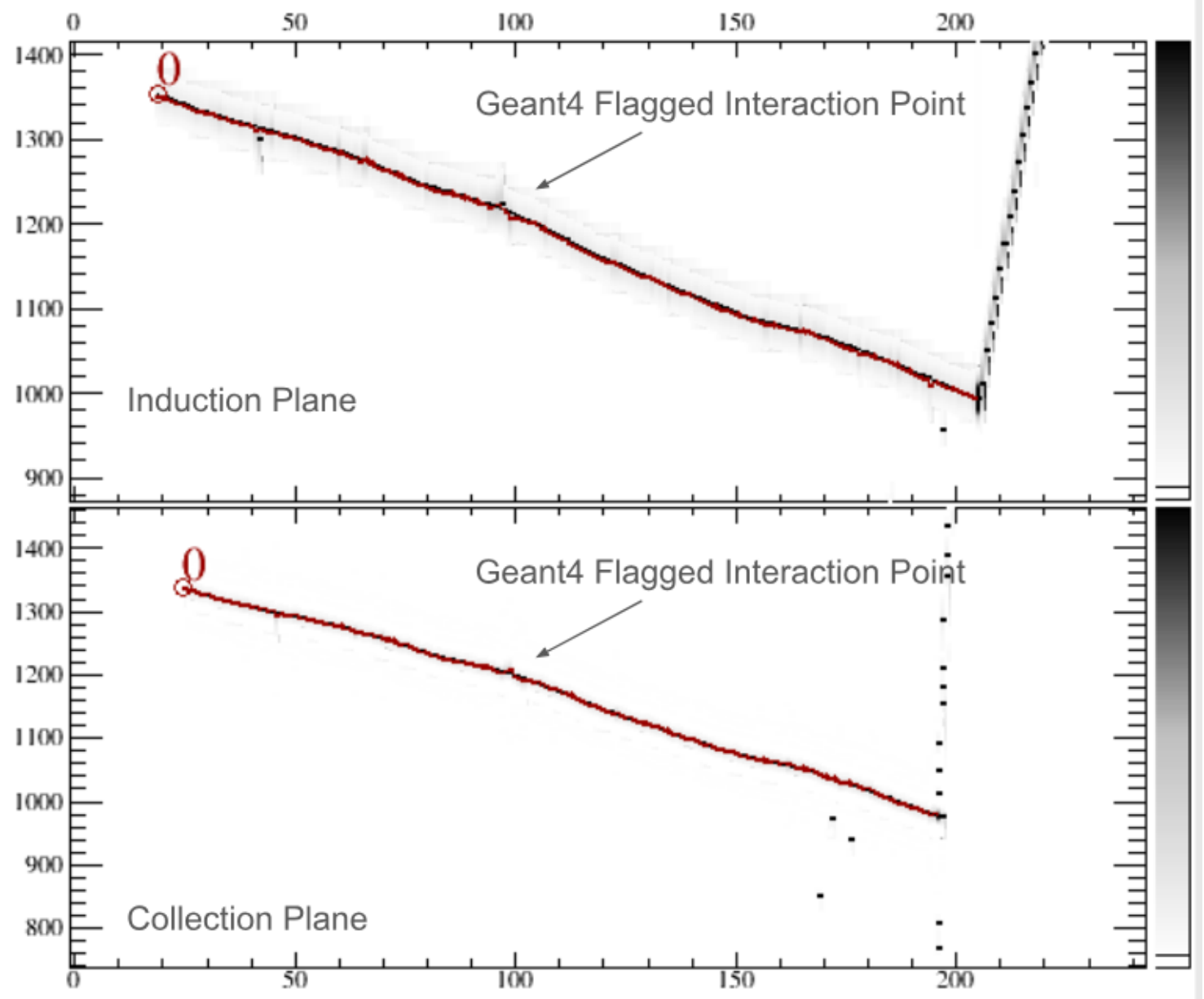

Figure B.2: Example of a shallow angle hadronic interaction "missed" by the TrajCluster. 


\section{Appendix C}

\section{Energy Calibration}

The scope of the energy calibration is to identify the factors which convert the charge collected (dQ) to the energy deposited in the chamber (dE). As described in Section 2.1.5, this is a multi-step procedure. In LArIAT, we first correct the raw charge by the electronic noise on the considered wire [78, then by the electron lifetime [73], and then by the recombination using the ArgoNeut recombination values. Lastly, we apply overall calibration of the energy, i.e., we determine the "calorimetry constants" using the procedure described in this section.

We independently determine the calorimetry constants for Data and Monte Carlo in the LArIAT Run-II Data samples using a parametrization of the stopping power (a.k.a. energy deposited per unit length, $d E / d X$ ) as a function of momentum. This is done by comparing the stopping power measured on reconstructed quantities against the Bethe-Bloch theoretical prediction for various particle species (see Equation 2.1). We obtain the theoretical expectation for the $d E / d X$ most probable value of pions $(\pi)$, muons $(\mu)$, kaons $(K)$, and protons $(p)$ in the momentum range most relevant for LArIAT (Figure C.1) using the tables provided by the Particle Data Group 36 for liquid argon [101].

The basic idea of this calibration technique is to utilize a sample of beamline 
events with known particle species and momentum to measure the $d E / d X$ of the corresponding tracks in the TPC. In particular, we decided to use positive pions as the calibration sample and samples from all the other particle species as a cross check. Once the $d E / d X$ of the positive pion sample has been measured at various momenta, we tune to calorimetry constants within the reconstruction software to align the measured values to match the theoretical ones found in Figure C.1. This is a new technique for calorimetry calibration in liquid argon developed by a joint ArgoNeut and LArIAT effort.

In data, we start by selecting a sample of beamline positive pion beamline candidates without any restriction on their measured momentum ${ }^{1}$. We then apply the WC2TPC match and subtract the energy loss upstream to the TPC front face, determining the momentum at the TPC front face. For each surviving pion candidate, we measure the $d E / d x$ at each of the first 12 spacepoints associated the $3 \mathrm{D}$ reconstructed track, corresponding to a $\sim 5 \mathrm{~cm}$ portion. These $d E / d X$ measurements are then put into a histogram that corresponds to measured momentum of the track. The $d E / d X$ histograms are sampled every $50 \mathrm{MeV} / \mathrm{c}$ in momentum (e.g. $150 \mathrm{MeV} / \mathrm{c}$ $<P<200 \mathrm{MeV} / \mathrm{c}, 200 \mathrm{MeV} / \mathrm{c}<P<250 / \mathrm{c} \mathrm{MeV}$, etc...). This process of selecting, sampling, and recording the $d E / d X$ for various momentum bins is repeated over the entire sample of events, allowing us to collect sufficient statistic in most of the momentum bins between $150 \mathrm{MeV} / \mathrm{c}$ and $1100 \mathrm{MeV} / \mathrm{c}$. On average, pions and muons only lose $\sim 10 \mathrm{MeV}$ in this $5 \mathrm{~cm}$ section of the track and protons lose $\sim 20 \mathrm{MeV}$. Thus choosing $50 \mathrm{MeV} / \mathrm{c}$ size bins for our histograms covers the energy spread within those bins due to energy loss from ionization for all the particle species identifiable in the beamline. Each $50 \mathrm{MeV} / \mathrm{c}$ momentum binned $d E / d X$ histogram is now fit with a simple Landau function. The most probable value (MPV) and the associated error on the MPV from the fit are extracted and plotted against the theoretical prediction

1. It should be noted that some muon and position contamination is present in the $\pi^{+}$sample. 
Figure C.1. Depending on the outcome of the data-prediction comparison, we modify the calorimetry constants and we repeat the procedure until a qualitative agreement is achieved. We perform this tuning for the collection and induction plane separately. As a cross check to the calorimetry constants determined using the positive pions, we lock the constants and plot the $d E / d x$ versus momentum distribution of all the other particle species identifiable in the beamline data $(\pi / \mu / e, \mathrm{~K}, \mathrm{p}$, in both polarities) against the corresponding Bethe-Bloch prediction. The agreement between data from the other particle species and the predictions is the expected result of this cross check. The results of the tuning and cross check for Run-II data on the collection plane is shown in Figure C.2 negative polarity data on top, positive polarity data on the bottom.

In $\mathrm{MC}$, we simulate the corresponding positive pion sample with the DDMC (see section 5.2.2 and follow the same steps as in data. More details on the calorimetry tuning can be found in [55].

After the calibration is done separately on data and MC, we can compare the resulting $\mathrm{dE} / \mathrm{dX}$ distributions; this is done for the set of pion beamline candidates and pion $\mathrm{MC}$ used in the cross section analysis on the left side of Figure C.3. On the right side of the same figure, we report the data-MC comparison for the kaon sample used in the cross section analysis. The distributions are fitted with simple Landau functions. As expected, the Landau MPV for data is consistent with the MC MPV for both pions and kaons. For both the pion and the kaon case, the width of the Landau is a bit wider in data than in MC; this difference might be due to an underestimate of the electronic noise in the MC simulation. 


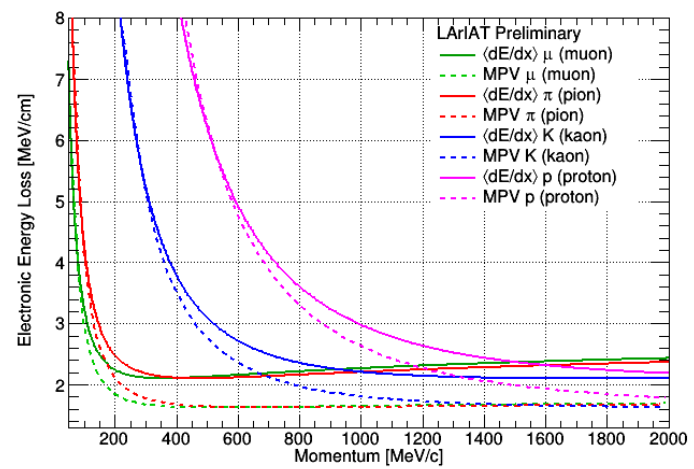

Figure C.1: Stopping power for pions, muons, kaons, and protons in liquid argon over the momentum range most relvant for LArIAT according to the Beth-Bloch equation. The solid lines represent the prediction for the mean energy $d E / d X$, while the dashed lines are the predictions for the MPV.
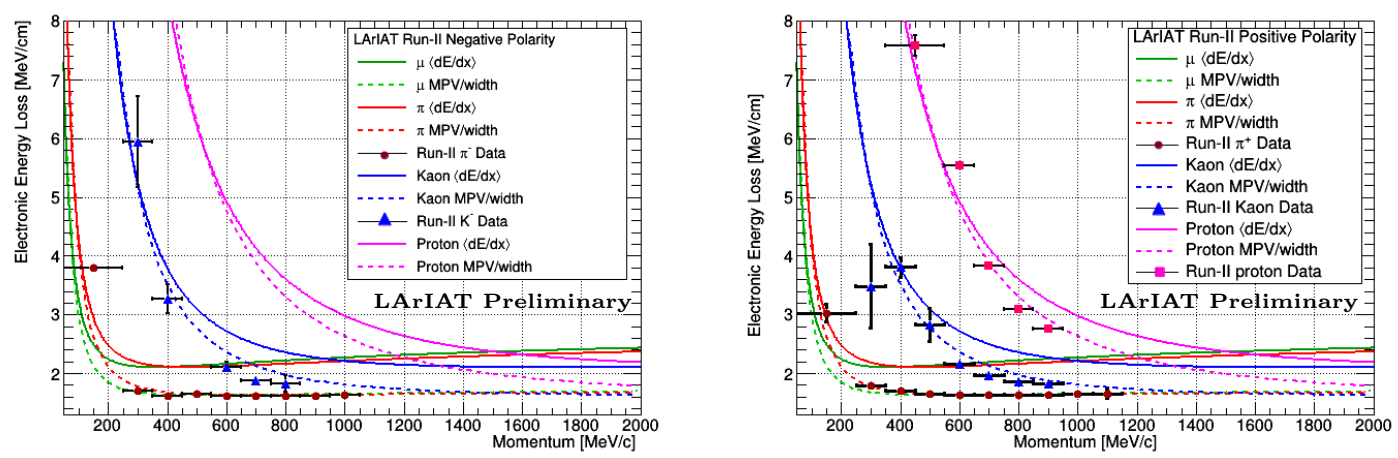

Figure C.2: Stopping power versus Momentum for Run-II negative (top) and positive (bottom) polarity data. We achieve the agreement between the Bethe-Bloch predictions and the distribution obtained with of the positive pions (top plot, red dots) by tuning the calorimetry constants. Once the calorimetry constants are locked in, the agreement between the other particle species and the Bethe-Bloch predictions follows naturally. 

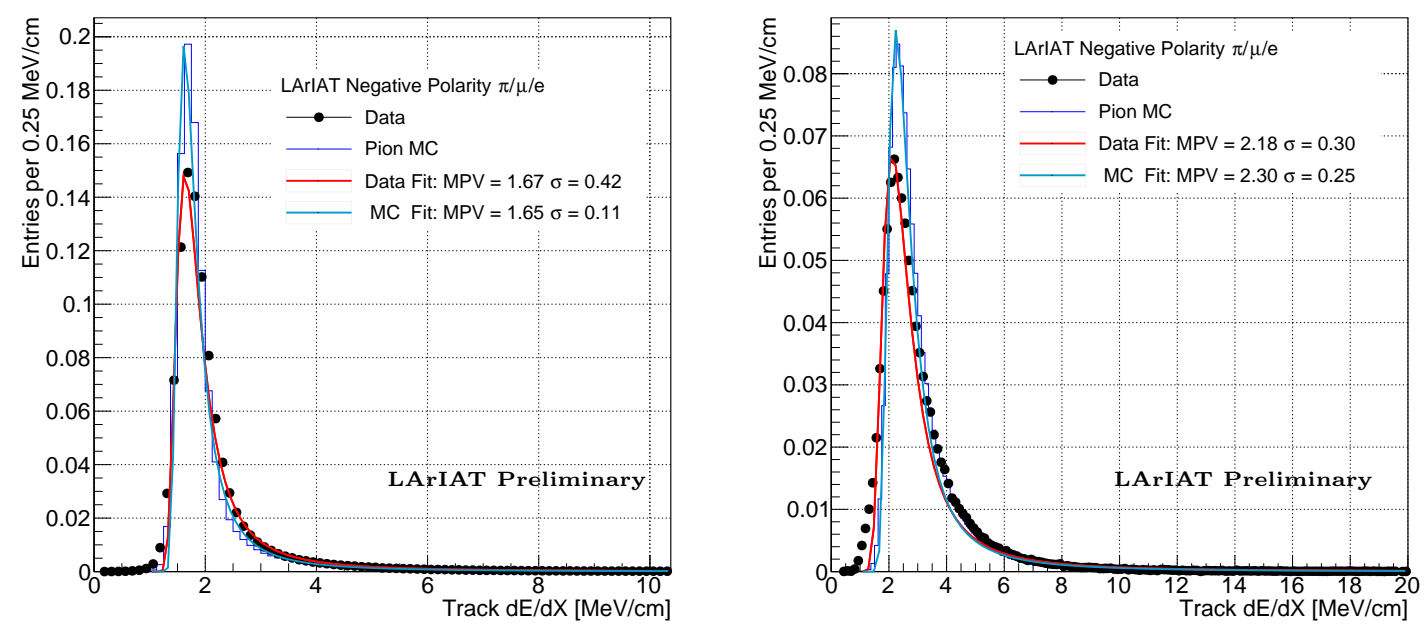

Figure C.3: Left: $\mathrm{dE} / \mathrm{dx}$ distribution for $\pi^{-} / \mu^{-} / e^{-}$data (black) and Pion MC (blue). The Landau fit for data is shown in red, the one for $\mathrm{MC}$ in teal. Right: $\mathrm{dE} / \mathrm{dx}$ distribution for $K^{+}$data (black) and Kaon MC (blue). The Landau fit for data is shown in red, the one for MC in teal. All the distributions are area normalized. 


\section{Bibliography}

[1] A. Agresti. Categorical Data Analysis. Wiley Series in Probability and Statistics. Wiley, 2013.

[2] B. Baller. Trajcluster user guide. Technical report, 2016. https://cdcvs. fnal.gov/redmine/documents/1026.

[3] G. Barker. Neutrino event reconstruction in a liquid argon TPC. Journal of Physics: Conference Series, 308:012015, 2011.

[4] J. B. Birks. Scintillations from organic crystals: Specific fluorescence and relative response to different radiations. Proceedings of the Physical Society. Section A, 64(10):874, 1951.

[5] CAEN. CAEN V1495 Data Sheet. Technical report, 2018. http://www.caen. it/csite/CaenProd. jsp?idmod=484\&parent=11.

[6] CAEN. CAEN V1740 Data Sheet. Technical report, 2018. http://www.caen. it/csite/CaenProd. jsp?parent=11\&idmod=591.

[7] D. Casper. The nuance neutrino physics simulation, and the future. Nuclear Physics B - Proceedings Supplements, 112(1-3):161-170, 2002.

[8] E. Church. LArSoft: A Software Package for Liquid Argon Time Projection Drift Chambers. ArXiv, physics.ins-de/1311.6774, 2013. 
[9] ATLAS Collaboration. Observation of a new particle in the search for the standard model higgs boson with the ATLAS detector at the LHC. Physics Letters B, 716(1):1-29, 2012.

[10] CMS Collaboration. Observation of a new boson at a mass of $125 \mathrm{GeV}$ with the CMS experiment at the LHC. Physics Letters B, 716(1):30 - 61, 2012.

[11] LArIAT Collaboration. The Liquid Argon In A Testbeam (LArIAT) Experiment. Technical report, In Preparation 2018.

[12] Nova Collaboration. Constraints on oscillation parameters from $\nu_{e}$ appearance and $\nu_{\mu}$ disappearance in no $\nu$ a. Phys. Rev. Lett., 118:231801, 2017.

[13] Super-Kamiokande Collaboration. Search for proton decay via $p \rightarrow e^{+} \pi^{0}$ and $p \rightarrow \mu^{+} \pi^{0}$ in 0.31 megaton $\cdot$ years exposure of the Super-Kamiokande water Cherenkov detector. Phys. Rev. D, 95:012004, 2017.

[14] M. A. Leigui de Oliveira. Expression of Interest for a Full-Scale Detector Engineering Test and Test Beam Calibration of a Single-Phase LAr TPC. Technical Report CERN-SPSC-2014-027. SPSC-EOI-011, CERN, Geneva, 2014.

[15] A. Aguilar-Arevalo et al. Evidence for neutrino oscillations from the observation of anti-neutrino(electron) appearance in a anti-neutrino(muon) beam. Phys. Rev., D64:112007, 2001.

[16] A. Aguilar-Arevalo et al. Improved Search for $\bar{\nu}_{\mu} \rightarrow \bar{\nu}_{e}$ Oscillations in the MiniBooNE Experiment. Phys. Rev. Lett., 110:161801, 2013.

[17] A. Bodek et al. Further studies of fermi-motion effects in lepton scattering from nuclear targets. Phys. Rev. D, 24:1400-1402, 1981. 
[18] A. Bueno et al. Nucleon decay searches with large liquid argon TPC detectors at shallow depths: atmospheric neutrinos and cosmogenic backgrounds. Journal of High Energy Physics, 2007(04):041-041, 2007.

[19] A. Cervera et al. Golden measurements at a neutrino factory. Nuclear Physics $B, 579(1-2): 17-55,2000$.

[20] A. Ereditato et al. Design and operation of ARGONTUBE: a $5 \mathrm{~m}$ long drift liquid argon TPC. Journal of Instrumentation, 8(07):P07002, 2013.

[21] A. Petrov et al. Java synoptic toolkit. Technical report, 2010. http: //synoptic.fnal.gov/doc/readme.txt.

[22] A. S. Carroll et al. Pion-nucleus total cross sections in the $(3,3)$ resonance region. Phys. Rev. C, 14:635-638, 1976.

[23] A.A. Aguilar-Arevalo et al. The MiniBooNE detector. Nuclear Instruments and Methods in Physics Research Section A: Accelerators, Spectrometers, Detectors and Associated Equipment, 599(1):28 - 46, 2009.

[24] A.S. Clough et al. Pion-nucleus total cross sections from 88 to $860 \mathrm{MeV}$. Nuclear Physics B, 76(1):15-28, 1974.

[25] B. Borut et al. Threshold corrections to dimension-six proton decay operators in non-minimal SUSY SU(5) GUTs. Nuclear Physics B, 910:1 - 22, 2016.

[26] B. J. P. Jones et al. A measurement of the absorption of liquid argon scintillation light by dissolved nitrogen at the part-per-million level. Journal of Instrumentation, 8(07):P07011, 2013.

[27] B.W. Allardyce et al. Pion reaction cross sections and nuclear sizes. Nuclear Physics A, 209(1):1 - 51, 1973. 
[28] C. Adams et al. The Long-Baseline Neutrino Experiment: Exploring Fundamental Symmetries of the Universe. 2013.

[29] C. Anderson et al. The ArgoNeuT Detector in the NuMI Low-Energy beam line at Fermilab. JINST, 7:P10019, 2012.

[30] C. Andreopoulos et al. The GENIE Neutrino Monte Carlo Generator. Nucl. Instrum. Meth., A614:87-104, 2010.

[31] C. Athanassopoulos et al. The liquid scintillator neutrino detector and LAMPF neutrino source. Nuclear Instruments and Methods in Physics Research Section A: Accelerators, Spectrometers, Detectors and Associated Equipment, 388(12):149-172, 1997.

[32] C. Athanassopoulos et al. Evidence for $\mathrm{nu}(\mathrm{mu}) \rightarrow \mathrm{nu}(\mathrm{e})$ neutrino oscillations from LSND. Phys. Rev. Lett., 81:1774-1777, 1998.

[33] C. E. Aalseth et al. DarkSide-20k: A 20 tonne two-phase LAr TPC for direct dark matter detection at LNGS. The European Physical Journal Plus, 133(3), 2018.

[34] C. Green et al. The Art Framework. J. Phys. Conf. Ser., 396:022020, 2012.

[35] C. Juszczak et al. Simulations from a new neutrino event generator. Nuclear Physics B - Proceedings Supplements, 159:211-216, 2006.

[36] C. Patrignani et al. Review of Particle Physics. Chin. Phys., C40(10):100001, 2016.

[37] C. S. Wu et al.. Experimental test of parity conservation in beta decay. Phys. Rev., 105:1413-1415, 1957.

[38] C. Wilkin et al. A comparison of pi+ and pi- total cross-sections of light nuclei near the 3-3 resonance. Nucl. Phys., B62:61-85, 1973. 
[39] D. Ashery et al. True absorption and scattering of pions on nuclei. Phys. Rev. C, 23:2173-2185, 1981 .

[40] D. Drakoulakos et al. Proposal to perform a high-statistics neutrino scattering experiment using a fine-grained detector in the NuMI beam. 2004.

[41] D. H. Wright et al. The Geant4 Bertini Cascade. Nucl. Instrum. Meth., A804:175-188, 2015.

[42] D. Lee et al. Predictions for the proton lifetime in minimal nonsupersymmetric so(10) models: An update. Phys. Rev. D, 51:229-235, 1995.

[43] D.V Bugg et al. Kaon-nucleon total cross sections from 0.6 to $2.65 \mathrm{GeV} / \mathrm{MeV}$ c. Phys. Rev., 168:1466-1475, 1968.

[44] E. Friedman et al. K+ nucleus reaction and total cross-sections: New analysis of transmission experiments. Phys. Rev., C55:1304-1311, 1997.

[45] E. Morikawa et al. Argon, krypton, and xenon excimer luminescence: From the dilute gas to the condensed phase. The Journal of Chemical Physics, 91(3):1469-1477, 1989.

[46] E. Torleif et al. Pions and Nuclei (The International Series of Monographs on Physics). Oxford University Press, 1988.

[47] F. Binon et al. Scattering of negative pions on carbon. Nuclear Physics B, 17(1):168 - 188, 1970.

[48] F. Cavanna et al. LArIAT: Liquid Argon In A Testbeam. arXiv, physics.ins$\operatorname{det} / 1406.5560,2014$.

[49] F M Newcomer et al. A fast, low power, amplifier-shaper-discriminator for high rate straw tracking systems. IEEE Transactions on Nuclear Science, 40(4):630636, 1993. 
[50] F.J. Hasert et al. Observation of neutrino-like interactions without muon or electron in the Gargamelle neutrino experiment. Physics Letters B, 46(1):138 $-140,1973$.

[51] H. Geiger et al. On a diffuse reflection of the formula-particles. Proceedings of the Royal Society A: Mathematical, Physical and Engineering Sciences, 82(557):495-500, 1909 .

[52] H. Georgi et al. Unity of all elementary-particle forces. Phys. Rev. Lett., 32:438$441,1974$.

[53] H. Minakata et al. Neutrino mixing and quark-lepton complementarity. Phys. Rev. D, 70:073009, 2004.

[54] J. A. Formaggio et al. From eV to EeV: Neutrino cross sections across energy scales. Rev. Mod. Phys., 84:1307-1341, 2012.

[55] J. Asaadi et al. Determination of the electron lifetime in LArIAT. Technical report, 2017. https://lartpc-docdb.fnal.gov/cgi-bin/private/ ShowDocument?docid=2513.

[56] J. L. Lopez et al. Flipped SU(5): Origins and recent developments. In 15th Johns Hopkins Workshop on Current Problems in Particle Theory: Particle Physics from Underground to Heaven Baltimore, Maryland, August 26-28, 1991, pages 277-297, 1991.

[57] J. Thomas et al. Recombination of electron-ion pairs in liquid argon and liquid xenon. Phys. Rev. A, 36:614-616, 1987.

[58] K. Abe et al. Search for proton decay via $p \rightarrow \nu K^{+}$using 260 kiloton $\cdot$ year data of super-kamiokande. Phys. Rev. D, 90:072005, 2014. 
[59] K. Abe et al. Combined analysis of neutrino and antineutrino oscillations at t2k. Phys. Rev. Lett., 118:151801, 2017.

[60] K. Nakamura et al. Neutrino Mass, Mixing and Oscillations. Chin. Phys., C40(10):100001, 2016.

[61] L. Aliaga et al. Minerva neutrino detector response measured with test beam data. Nuclear Instruments and Methods in Physics Research Section A: Accelerators, Spectrometers, Detectors and Associated Equipment, 789:28 - 42, 2015.

[62] M. Adamowski et al. The liquid argon purity demonstrator. Journal of Instrumentation, 9(07):P07005, 2014.

[63] M. Antonello et al. Precise 3d track reconstruction algorithm for the ICARUS t600 liquid argon time projection chamber detector. Advances in High Energy Physics, 2013:1-16, 2013.

[64] M. Antonello et al. A Proposal for a Three Detector Short-Baseline Neutrino Oscillation Program in the Fermilab Booster Neutrino Beam. 2015.

[65] M. G. Boulay et al. Direct WIMP detection using scintillation time discrimination in liquid argon. arXiv, astro-ph/0411358, 2004.

[66] N. Ishida et al. Attenuation length measurements of scintillation light in liquid rare gases and their mixtures using an improved reflection suppresser. Nuclear Instruments and Methods in Physics Research Section A: Accelerators, Spectrometers, Detectors and Associated Equipment, 384(2-3):380-386, 1997.

[67] N. Yahlali et al. Imaging with sipms in noble-gas detectors. Journal of Instrumentation, 8(01):C01003, 2013. 
[68] P. Vilain et al. Coherent single charged pion production by neutrinos. Physics Letters B, 313(1-2):267-275, 1993.

[69] Q. Shafi et al. Neutrino democracy, fermion mass hierarchies, and proton decay from 5D SU(5). Phys. Rev. D, 67:075007, 2003.

[70] R. Acciarri et al. Effects of nitrogen contamination in liquid argon. Journal of Instrumentation, 5(06):P06003, 2010.

[71] R. Acciarri et al. Demonstration and Comparison of Operation of Photomultiplier Tubes at Liquid Argon Temperature. JINST, 7:P01016, 2012.

[72] R. Acciarri et al. A study of electron recombination using highly ionizing particles in the argoneut liquid argon tpc. Journal of Instrumentation, 8(08):P08005, 2013.

[73] R. Acciarri et al. Determination of the electron lifetime in LArIAT. Technical report, 2016. https://lartpc-docdb.fnal.gov/cgi-bin/private/ RetrieveFiledocid=1804\&filename=LArIAT_Electron_Lifetime.pdf\& version $=2$.

[74] R. Acciarri et al. Convolutional neural networks applied to neutrino events in a liquid argon time projection chamber. Journal of Instrumentation, 12(03):P03011, 2017.

[75] R. Acciarri et al. Design and Construction of the MicroBooNE Detector. JINST, 12(02):P02017, 2017.

[76] R. Acciarri et al. Design and construction of the MicroBooNE detector. Journal of Instrumentation, 12(02):P02017-P02017, 2017. 
[77] R. Acciarri et al. First Observation of Low Energy Electron Neutrinos in a Liquid Argon Time Projection Chamber. Phys. Rev., D95(7):072005, 2017. [Phys. Rev.D95,072005(2017)].

[78] R. Acciarri et al. Investigation of the non-uniformity observed in the wire response to charge in LArIAT Run 1. Technical report, 2017. https: //lartpc-docdb.fnal.gov/cgi-bin/private/ShowDocument?docid=1994.

[79] R. K. Teague et al. Refractive index and the lorentz-lorenz function for gaseous and liquid argon, including a study of the coexistence curve near the critical state. The Journal of Chemical Physics, 48(11):4973-4984, 1968.

[80] S. Amoruso et al. Study of electron recombination in liquid argon with the ICARUS TPC. Nucl. Instrum. Meth., A523:275-286, 2004.

[81] S. Dell'Oro et al. Neutrinoless double beta decay: 2015 review. Advances in High Energy Physics, 2016:1-37, 2016.

[82] S. Dimopoulos et al. Proton Decay in Supersymmetric Models. Phys. Lett., B112:133, 1982.

[83] S. Hansen et al. Fermilab test beam multi-wire proportional chamber tracking system upgrade. 2014. International Conference on Technology and Instrumentation in Particle Physics (TIPP 2014).

[84] S. Pascoli et al. Leptogenesis and low energy CP-violation in neutrino physics. Nuclear Physics B, 774(1):1 - 52, 2007.

[85] S. Ritz et al. Building for Discovery: Strategic Plan for U.S. Particle Physics in the Global Context. 2014.

[86] S.E. Derenzo et al. Test of a liquid argon chamber with $20 \mu \mathrm{m}$ RMS resolution. Nuclear Instruments and Methods, 122:319 - 327, 1974. 
[87] SLD Electroweak Group et al. Precision electroweak measurements on the Z resonance. Physics Reports, 427(5):257 - 454, 2006.

[88] V. Flaminio et al. Compilation of Cross Sections I: $\pi^{+}$and $\pi^{-}$Induced Reactions. CERN-HERA, pages 83-01, 1983.

[89] V. Flaminio et al. Compilation of Cross Sections II: $K^{+}$and $K^{-}$Induced Reactions. CERN-HERA, pages 83-02, 1983.

[90] V. Lucas et al. Nucleon decay in a realistic so(10) susy gut. Phys. Rev. D, 55:6986-7009, 1997.

[91] V. Takhistov et al. Search for nucleon and dinucleon decays with an invisible particle and a charged lepton in the final state at the super-kamiokande experiment. Phys. Rev. Lett., 115:121803, 2015.

[92] V.M. Gehman et al. Fluorescence efficiency and visible re-emission spectrum of tetraphenyl butadiene films at extreme ultraviolet wavelengths. Nuclear Instruments and Methods in Physics Research Section A: Accelerators, Spectrometers, Detectors and Associated Equipment, 654(1):116 - 121, 2011.

[93] W. H. Lippincott et al. Scintillation time dependence and pulse shape discrimination in liquid argon. Phys. Rev. C, 78:035801, 2008.

[94] W. M. Burton et al. Fluorescence of tetraphenyl-butadiene in the vacuum ultraviolet. Applied Optics, 12(1):87, 1973.

[95] W.J. Willis et al. Liquid-argon ionization chambers as total-absorption detectors. Nuclear Instruments and Methods, 120(2):221 - 236, 1974.

[96] H. Fenker. Standard beam PWC for Fermilab. Technical report, Fermi National Accelerator Lab., Batavia, IL (USA), 1983. 
[97] H. Fesbach. Theoretical nuclear physics: Nuclear reactions. John Wiley \& Sons, Inc., New York, NY (United States), 1992.

[98] W. Gajewski et al. Search for proton decay into $e^{+}+\pi^{0}$ in the IMB-3 detector. Phys. Rev. D, 42:2974-2976, 1990.

[99] Technical report. Glassman High Voltage, Inc., Precision Regulated High Voltage DC Power Supply.

[100] D. S. Gorbunov. Sterile neutrinos and their role in particle physics and cosmology. Physics-Uspekhi, 57(5):503, 2014.

[101] Particle Data Group. Energy Loss for Liquid Argon. Technical report. http://pdg.lbl.gov/2017/AtomicNuclearProperties/MUE/muE_ liquid_argon.txt.

[102] J. Harada. Non-maximal $\theta_{23}$, large $\theta_{13}$ and tri-bimaximal $\theta_{12}$ via quarklepton complementarity at next-to-leading order. EPL (Europhysics Letters), $103(2): 21001,2013$.

[103] W. Hermann. Gravitation and the electron. Proceedings of the National Academy of Sciences of the United States of America, 15(4):323-334, 1929.

[104] P.W. Higgs. Broken symmetries, massless particles and gauge fields. Physics Letters, 12(2):132-133, 1964.

[105] H. J. Hilke. Time projection chambers. Reports on Progress in Physics, $73(11): 116201,2010$.

[106] G. Jaffé. Zur theorie der ionisation in kolonnen. Annalen der Physik, 347(12):303-344, 1913. 
[107] C. Jarlskog. A basis independent formulation of the connection between quark mass matrices, CP violation and experiment. Zeitschrift für Physik C Particles and Fields, 29(3):491-497, 1985.

[108] B.J.P. Jones. Sterile Neutrinos in Cold Climates. PhD thesis, MIT, 2015.

[109] D. I. Kazakov. Beyond the standard model: In search of supersymmetry. In 2000 European School of high-energy physics, Caramulo, Portugal, 20 Aug-2 Sep 2000: Proceedings, pages 125-199, 2000.

[110] E. Majorana. Teoria simmetrica dell'elettrone e del positrone. Il Nuovo Cimento, 14(4):171-184, 1937.

[111] M. Mooney. The microboone experiment and the impact of space charge effects. arXiv. physics.ins-det/1511.01563, 2015.

[112] E. Noether. Invariant variation problems. Transport Theory and Statistical Physics, 1(3):186-207, 1971.

[113] I. Nutini. Study of charged particles interaction processes on ar in the $0.2-2.0$ $\mathrm{GeV}$ energy range through combined information from ionization free charge and scintillation light. Technical report, 2015. https://doi.org/10.2172/ 1221325 .

[114] D. R. Nygren. The time projection chamber: A new $4 \pi$ detector for charged particles. Technical report, 1974. http://1ss.fnal.gov/conf/C740805/p58. $\mathrm{pdf}$.

[115] L. Onsager. Initial recombination of ions. Phys. Rev., 54:554-557, 1938.

[116] B. Pontecorvo. Neutrino Experiments and the Problem of Conservation of Leptonic Charge. Sov. Phys. JETP, 26:984-988, 1968. [Zh. Eksp. Teor. Fiz.53,1717(1967)]. 
[117] M. Raidal. Relation between the neutrino and quark mixing angles and grand unification. Phys. Rev. Lett., 93:161801, 2004.

[118] C. Rubbia. The Liquid Argon Time Projection Chamber: A New Concept for Neutrino Detectors. 1977.

[119] L.M Saunders. Electromagnetic production of pions from nuclei. Nucl. Phys., B7: 293-310(1968)., 1968.

[120] G.L. Shaw and D.Y. Wong (editors). Pion-nucleon Scattering. John Wiley \& Sons Inc, 1969.

[121] W. Walkowiak. Drift velocity of free electrons in liquid argon. Nuclear Instruments and Methods in Physics Research Section A: Accelerators, Spectrometers, Detectors and Associated Equipment, 449(1-2):288-294, 2000.

[122] T. Yanagida. Horizontal symmetry and masses of neutrinos. Progress of Theoretical Physics, 64(3):1103-1105, 1980. 